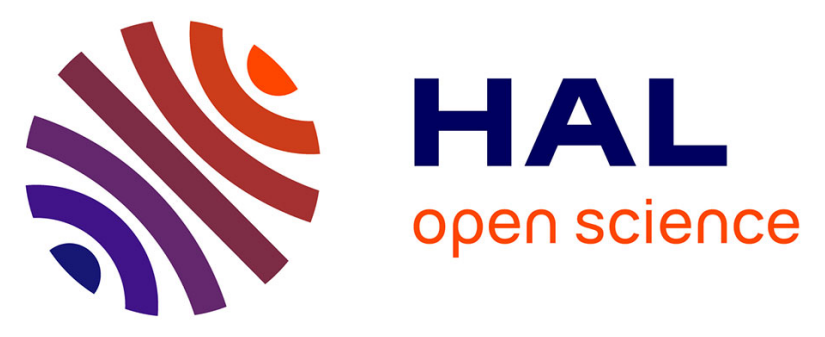

\title{
Recent advances in collisional effects on spectra of molecular gases and their practical consequences
}

Jean-Michel Hartmann, Ha Tran, Raymond Armante, Christian Boulet, Alain Campargue, Francois Forget, Livio Gianfrani, Iouli Gordon, Sandrine Guerlet, Magnus Gustafsson, et al.

\section{To cite this version:}

Jean-Michel Hartmann, Ha Tran, Raymond Armante, Christian Boulet, Alain Campargue, et al.. Recent advances in collisional effects on spectra of molecular gases and their practical consequences. Journal of Quantitative Spectroscopy and Radiative Transfer, 2018, 213, pp.178-227. 10.1016/j.jqsrt.2018.03.016 . hal-01833211v2

\section{HAL Id: hal-01833211}

https://hal-univ-rennes1.archives-ouvertes.fr/hal-01833211v2

Submitted on 11 Sep 2018

HAL is a multi-disciplinary open access archive for the deposit and dissemination of scientific research documents, whether they are published or not. The documents may come from teaching and research institutions in France or abroad, or from public or private research centers.
L'archive ouverte pluridisciplinaire HAL, est destinée au dépôt et à la diffusion de documents scientifiques de niveau recherche, publiés ou non, émanant des établissements d'enseignement et de recherche français ou étrangers, des laboratoires publics ou privés. 


\section{Highlights}

- A review on the progress made in the last decade on pressure effects on absorption shapes

- High accuracy measurement techniques

- Theories, models and available data for the absorption shapes

- Consequences for remote sensing, metrology and heat transfer applications

- Over 700 references

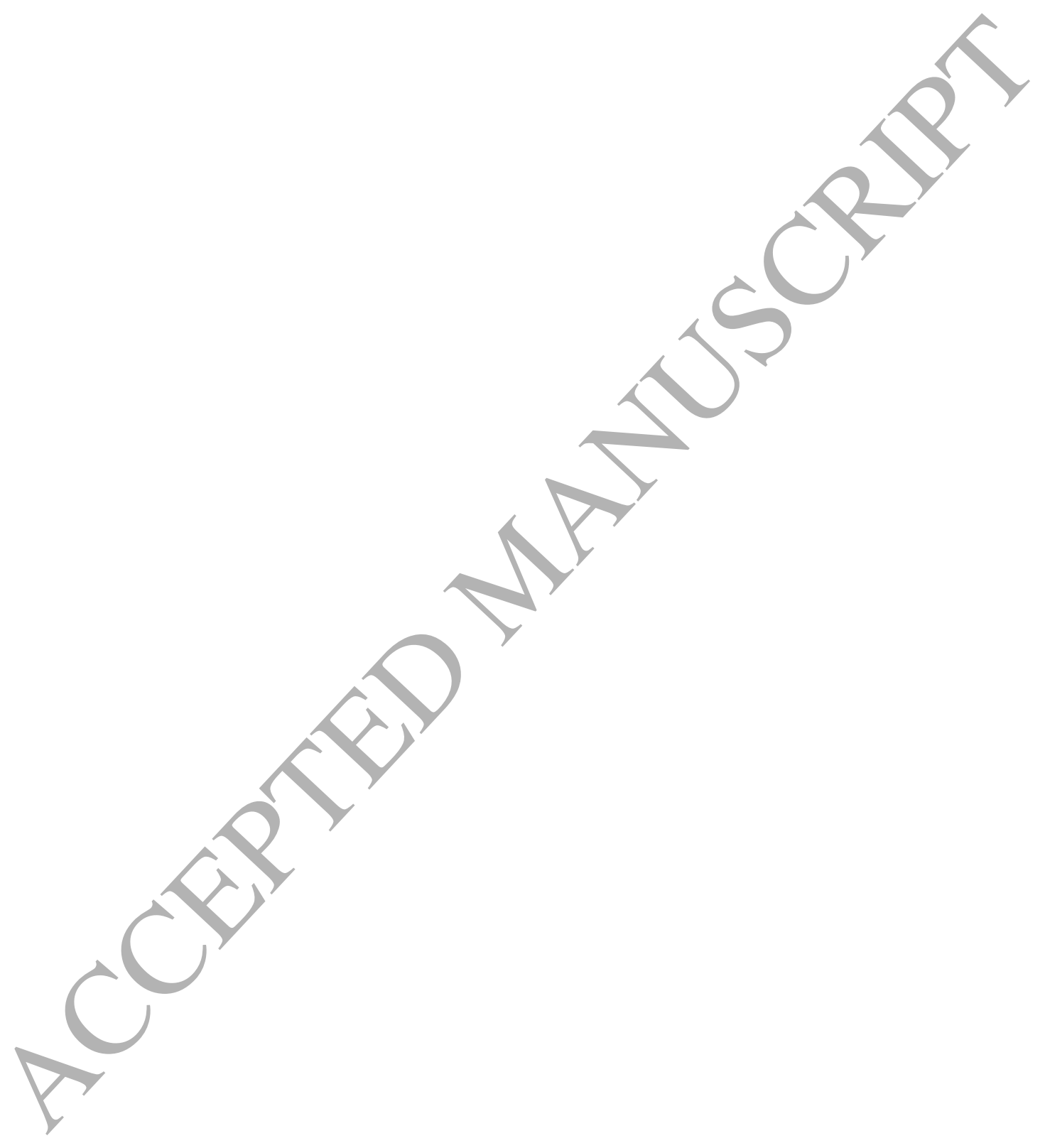




\title{
Recent advances in collisional effects on spectra of molecular gases and their practical consequences
}

\author{
Jean-Michel Hartmann ${ }^{\mathrm{a}, \#}$, Ha Tran ${ }^{\mathrm{b}}$, Raymond Armante ${ }^{\mathrm{a}}$, Christian Boulet ${ }^{\mathrm{c}}$, \\ Alain Campargue ${ }^{\mathrm{d}}$, François Forget ${ }^{\mathrm{b}}$, Livio Gianfrani ${ }^{\mathrm{e}}$, Iouli Gordon ${ }^{\mathrm{f}}$, Sandrine Guerlet ${ }^{\mathrm{b}}$, \\ Magnus Gustafsson ${ }^{\mathrm{g}}$, Joseph T. Hodges ${ }^{\mathrm{h}}$, Samir Kassi $^{\mathrm{d}}$, Daniel Lisak ${ }^{\mathrm{i}}$, Franck Thibault ${ }^{\mathrm{j}}$, \\ Geoffrey C. Toon ${ }^{\mathrm{k}}$ \\ a Laboratoire de Météorologie Dynamique/IPSL, CNRS, École polytechnique, Sorbonne Université, \\ École normale supérieure, PSL Research University, F-91120 Palaiseau, France. \\ ${ }^{\#}$ Corresponding author: jean-michel.hartmann@1md.polytechnique.fr \\ baboratoire de Météorologie Dynamique/IPSL, CNRS, Sorbonne Université, École normale \\ supérieure, PSL Research University, École polytechnique, F-75005 Paris, France. \\ 'Institut des Sciences Moléculaires d'Orsay, CNRS, Université Paris-Sud, Université Paris-Saclay, \\ Orsay F-91405, France. \\ ${ }^{\mathrm{d}}$ Univ. Grenoble Alpes, CNRS, LIPhy, 38000 Grenoble, France. \\ eDipartimento di Matematica e Fisica, Università degli Studi della Campania "Luigi Vanvitelli" \\ Viale Lincoln 5, 81100 Caserta, Italy \\ ${ }^{\mathrm{f}}$ Harvard-Smithsonian Center for Astrophysics, Atomic and Molecular Physics Division, \\ Cambridge, MA 02138, USA. \\ ${ }^{\mathrm{g}}$ Department of Engineering Sciences and Mathematics, Luleå University of Technology, \\ SE-97187 Luleà, Sweden. \\ ${ }^{\mathrm{h}}$ National Institute of Standards and Technology, 100 Bureau Drive, Gaithersburg, MD 20899, USA. \\ ${ }^{\mathrm{i}}$ Institute of Physics, Faculty of Physics, Astronomy and Informatics, Nicolaus Copernicus University, \\ Grudziadzka 5, 87-100 Torun, Poland. \\ ${ }^{\mathrm{j}}$ Institut de Physique de Rennes, UMR CNRS 6251, Université de Rennes 1, Campus de Beaulieu, \\ Bât.11B, F-35042 Rennes, France. \\ ${ }^{\mathrm{k}}$ Jet Propulsion Laboratory, California Institute of Technology, Pasadena, California, USA.
}

\begin{abstract}
We review progress, since publication of the book "Collisional effects on molecular spectra: Laboratory experiments and models, consequences for applications" (Elsevier, Amsterdam, 2008), on measuring, modeling and predicting the influence of pressure (ie of intermolecular collisions) on the spectra of gas molecules. We first introduce recently developed experimental techniques of high accuracy and sensitivity. We then complement the above mentioned book by presenting the theoretical approaches, results and data proposed (mostly) in the last decade on the topics of isolated line shapes, line-broadening and -shifting, line-mixing, the far wings and associated continua, and collision-induced absorption. Examples of recently demonstrated consequences of the progress in the description of spectral shapes for some practical applications (metrology, probing of gas media, climate predictions) are then given. Remaining issues and directions for future research are finally discussed.
\end{abstract}

Key words: pressure effects on spectral shapes; experimental techniques; theories and models; available data; consequences for applications 


\section{List of acronyms}

\section{Table of contents}

\section{Introduction}

\section{Laboratory experimental techniques}
2.1 Introduction
2.2 Cavity Enhanced Absorption Spectroscopy (CEAS)
2.3 Cavity Ring-Down Spectroscopy (CRDS)
2.4 Frequency-comb assisted methods
2.5 Cavity mode-width and mode-dispersion spectroscopies
2.6 Direct frequency-comb spectroscopy
2.7 Cavity-enhanced direct frequency-comb spectroscopy
2.8 Dual-laser absorption spectroscopy
2.9 Fourier-transform spectroscopy (FTS) methods
2.10 Advances in coherent terahertz spectroscopy

\section{Theories, models, results}

3.1 Introduction and selection criteria

\subsection{Isolated line shapes}

3.2.1 Direct and empirically corrected predictions from Molecular Dynamics Simulations

3.2.2 Using a kinetic equation

3.2.2a The Keilson-Storer $(K S)$ model for the velocity collision kernel

3.2.2b The billiard-ball (BB) approximation for the velocity collision kernel

3.2.2c Velocity collision kernel calculated from CMDS

3.2.3 Relativistic and dispersion correction to line-shape models

3.2.4 Phenomenological line-shape models

3.2.4a The Hartmann-Tran profile

3.2.4b Temperature dependence of the line-shape parameters

3.2.4c Quadratic versus hypergeometric speed dependences

3.2.4d Kochanov's approach

$\underline{3.3 \text { Pressure-broadening and -shifting coefficients }}$

3.3.1 Quantal approaches

3.3.2 Refined semi-classical Robert-Bonamy formalism

3.3.3 Fully classical approach

3.3.4 Some recent applications of the "usual" Robert-Bonamy formalism

3.4 Line-mixing

3.4.1 Requantized Classical Molecular Dynamics Simulations

3.4.2 Quantal approaches

3.4.3 Refined semi-classical Robert-Bonamy formalism

3.4.4 Fully classical formalism

3.4.5 Dynamically-based scaling laws

3.4.6 Energy-gap fitting laws and state-to-state cross sections

3.4.7 The ovoid sphere model

3.4.8 Kochanov's approach

3.5 Far wings and associated continua

3.5.1 Direct predictions from Molecular Dynamics Simulations

3.5.2 Non Markovian Energy-Corrected Sudden approach

3.5.3 Asymptotic line shape and $\chi$-factor empirical models

3.5.4 The MT_CKD water-vapor continuum 
3.6 Collision-induced absorption (CIA)

3.6.1 Quantum scattering advances

3.6.2 Intra-collisional interference effects

3.6.3 Standard binary-collision treatments

3.6.4 Direct predictions from Molecular Dynamics Simulations

3.6.5 Integrated CIA intensities

\section{Available data}

$4.1 \quad$ Isolated lines

4.2 Line-mixing

$\underline{4.3}$ Far wings and associated continua

4.3.1 The water-vapor continua

4.3.1a The self-continuum

4.3.1b The foreign-continua

4.3.2 The $\mathrm{CO}_{2}$ far-wings continua

4.4 Collision-induced absorption

4.5 Spectroscopic databases

4.5.1 The HITRAN database

4.5.2 The GEISA database and 4A/OP code

5. Consequences for applications

5.1 Remote sensing

5.1.1 Probing atmospheres

5.1.1 a The Earth

5.1.1b Other planets

5.1.2 Probing combustion gases

5.1.3 Probing confined gases

5.2 Metrology

5.2.1 Doppler thermometry

5.2.2 Amount of gas metrology

5.3 Radiative heat transfer and climate modeling

5.3.1 Heat transfer in "industrial" devices.

5.3.2 Modeling atmospheres observed in the solar system

5.3.3 New challenges: simulating exoplanets and ancient atmospheres.

\section{Remaining issues and directions for future research}

$\underline{6.1 \text { Experiments }}$

$\underline{6.2}$ Theories and models

6.3 Databases

Acknowledgements

\section{References list}


List of acronyms

\begin{tabular}{|l|l|}
\hline \multicolumn{1}{|c|}{ Acronym } & \multicolumn{1}{|c|}{ Meaning } \\
\hline 1D-CMDS & One-Dimensional Cavity-Mode Dispersion Spectroscopy \\
\hline ACF & Auto-Correlation Function \\
\hline BB & Billiard-Ball \\
\hline BWO & Backward Wave Oscillator \\
\hline CAVIAR & $\begin{array}{l}\text { Continuum Absorption at Visible and Infrared wavelengths and its } \\
\text { Atmospheric Relevance }\end{array}$ \\
\hline CC & Close-Coupling \\
\hline CEAS & Cavity Enhanced Absorption Spectroscopy \\
\hline CIA & Collision-Induced Absorption \\
\hline CMDS & Classical Molecular Dynamics Simulations \\
\hline CMWS & Cavity Mode-Width Spectroscopy \\
\hline CRDS & Cavity Ring-Down Spectroscopy \\
\hline CS & Coupled-States \\
\hline CW & Continuous Wave \\
\hline DBT & Doppler Broadening Thermometry \\
\hline DCS & Dual Comb Spectroscopy \\
\hline DFBL & Distributed Feed Back diode Laser \\
\hline ECDL & External Cavity Diode Laser \\
\hline ECS & Energy-Corrected Sudden \\
\hline EOM & Electro-Optic phase Modulator \\
\hline FP & Fabry-Pérot \\
\hline FS-CRDS & Frequency-Stabilized CRDS \\
\hline FSR & Free Spectral Range \\
\hline FTS & Fourier Transform Spectrometer \\
\hline FWHM & Full Width at Half Maximumy \\
\hline GP & Galatry Profile \\
\hline HAPI & HITRAN Application Programming Interface \\
\hline HC & Hard Collision \\
\hline HTP & Hartmann-Tran Profile \\
\hline ICL & Interband Cascade Laser \\
\hline IOS & Infinite-Order Sudden \\
\hline KS & Keilson-Storer \\
\hline LM & Line-Mixing \\
\hline MT_CKD & Mlawer-Tobin_Clough-Kneizys-Davies \\
\hline NICE- & Noise-Immune Cavity-Enhanced Optical Heterodyne Molecular \\
OHMS & Spectroscopy \\
\hline OFC & Optical Frequency Combs \\
\hline OF-CEAS & Optical-Feedback Cavity-Enhanced Absorption Spectroscopy \\
\hline pCqSDHC & partially-Correlated quadratic-Speed-Dependent Hard Collision \\
\hline QCL & Quantum Cascade Laser \\
\hline RB & Robert-Bonamy \\
\hline RP & Rautian Profile \\
\hline SC & Soft Collision \\
\hline SDBB & Speed-Dependent Billiard-Ball \\
\hline SDVP & Speed-Dependent Voigt Profile \\
\hline SI & Système International d'Unités \\
\hline SNR & Signal-to-Noise Ratio \\
\hline
\end{tabular}




\begin{tabular}{|l|l|}
\hline TCCON & Total Carbon Column Observatory Network \\
\hline VIPA & Virtually Imaged Phased Array \\
\hline VMR & Volume Mixing Ratio \\
\hline VP & Voigt Profile \\
\hline
\end{tabular}

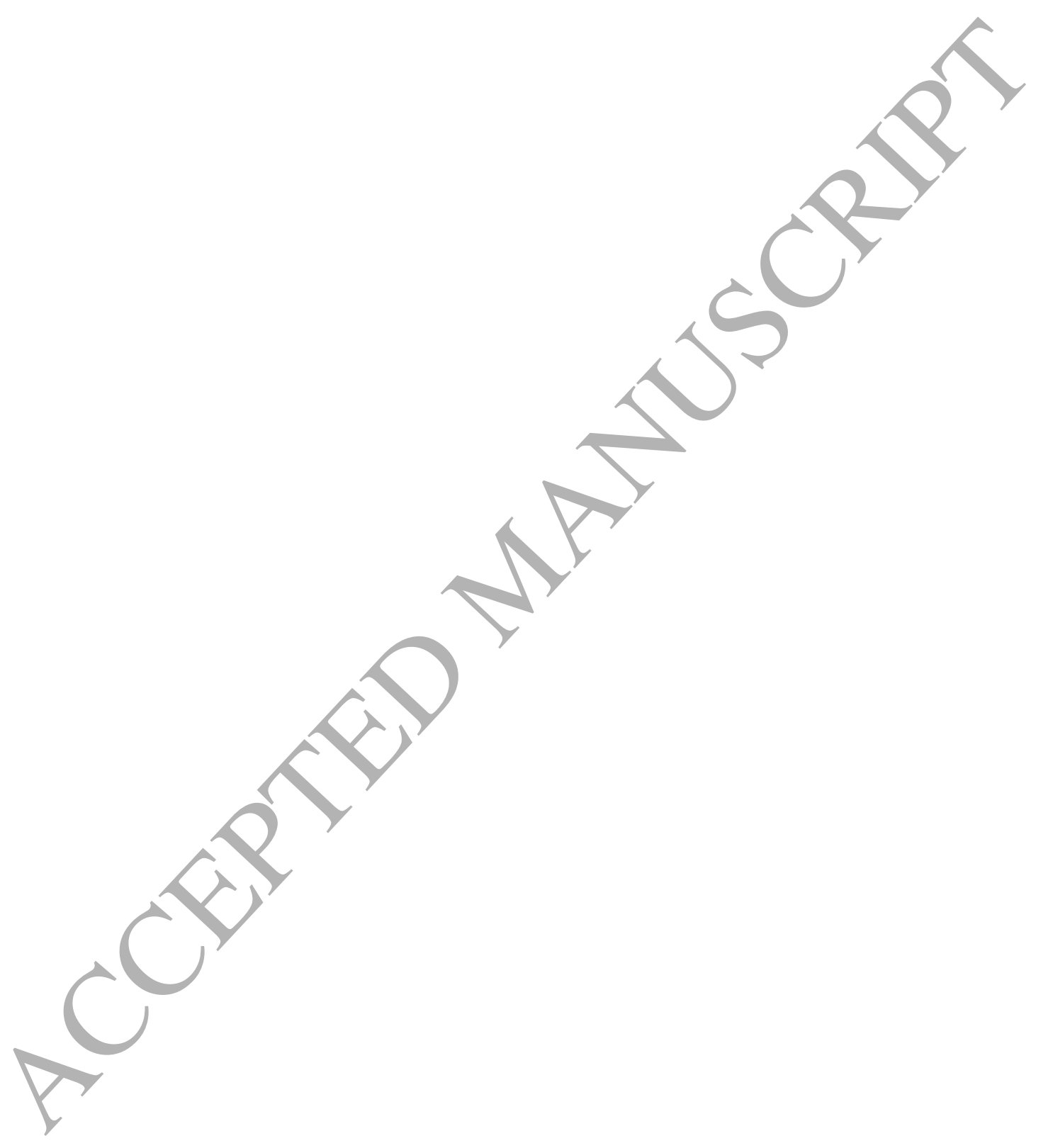




\section{Introduction}

In 2008, some authors of this paper completed a book [1] devoted to the effects of pressure (of intermolecular collisions) on the spectra of gas molecules. They reviewed the processes involved, the available theories, models and experimental results, and discussed the consequences for some practical applications. Considerable qualitative and quantitative progress has been made on all aspects of this subject that are discussed in this book since its publication a decade ago. The aim of the present review is to give the reader an overall view of these advances together with an update of the bibliography on the subject, including recent techniques for measurements of light absorption by gases. This rapidly growing field has many relevant publications, here we review those that we are aware of and that demonstrate new progress in one or more aspects of the research.

The remainder of this article is organized as follows: Section 2 is devoted to laboratory experimental techniques, a topic not treated in [1]. While it focuses mainly on recent developments based on the use of lasers and often of high finesse cavities, which enable measured absorption spectra with unprecedented high SNRs and accuracies, Fourier transform and terahertz techniques are also discussed. The new spectral-shape theories and models, as well as recently made comparisons between calculated and measured results are the subjects of Sec. 3. In the latter, the profiles of isolated lines, the pressure-broadening and shifting coefficients, line-mixing processes, the (far) line wings and continua, and collisioninduced absorption are successively considered (following chapters III-VI of [1]). Some recently made available spectral-shape parameters in these fields are presented in Sec. 4 together with the evolutions and current statuses of spectroscopic databases. The effects of collisions on molecular spectra are important to many applications, as discussed in Chapt. VII of [1]. Section 5 is devoted to the impact of achievements on this topic in a variety of other fields. These include metrology, the probing of various media including atmospheres, and radiative transfer and climate. Remaining problems and possible directions for future research are finally discussed in Sec. 6.

\section{Laboratory experimental techniques}

\subsection{Introduction}

Molecular spectra reveal collisional effects through changes in their shapes recorded along a frequency axis. To properly test the most advanced theories and models it is mandatory to minimize experimental biases, at least below the expected precision of the predictions. In addition, some applications (Sec. 5) require extremely reliable simulations or analysis of spectra. These constraints call for accuracy in both the frequency and absorption axes, which are intimately linked by the spectral profile, as well as for the accurate determination of the composition, temperature and pressure of the sample gas (Sec. 5.2.2). As an example, consider a direct absorption experiment in which a laser probes a gas and the transmission is recorded. Any uncontrolled laser-intensity noise would obviously convert into an effective absorption noise, impairing the fast and precise determination of a line contour. To some extent, this may be corrected for by stabilizing the instantaneous laser power that irradiates the gas sample, for example by using an acousto-optic modulator in a servo configuration [2]. However, while such a method can lead to baseline relative intensity noise levels approaching $10^{-5}$, going below this degree of intensity stabilization is practically impossible. In addition, fluctuations in the optical frequency of the probe laser that occur on time scales shorter than the characteristic data-acquisition rate are known as "frequency-jitter" and are manifest as noise in acquired spectra, particularly in regions of steep absorption. Worse, if the laser source, or the measurement of its optical frequency, slowly drifts during an experiment, the absorption spectrum is irreversibly deformed. Nevertheless, probe-laser jitter 
can be substantially reduced by active stabilization methods that incorporate a reference oscillator. These remarks show that an optimized classical spectrometer for absorption measurements should use an optical source having low amplitude and frequency noises, with a stable emission frequency that must be accurately monitored and determined.

Nowadays, the most accurate and sensitive techniques for absorption measurements use $\mathrm{CW}$ lasers as the light source and resonant optical cavities surrounding the sample gas. When compared to classical incoherent sources and pulsed lasers, CW lasers provide relatively low intensity noise and narrow emission widths capable of probing individual cavity resonances (modes). Resonant optical cavities comprise two or more high-reflectivity dielectric mirrors that sustain standing-wave or traveling-wave intra-cavity fields. Using such a resonator to enclose the gas sample increases the sensitivity by enhancing the path length of the photons trapped inside the cavity. This enhancement increases with the mirror reflectivities, as explained later. Also, because of the relatively long photon lifetime $(\tau)$ in the cavity and coherent nature of the excitation, these cavities are highly efficient frequency filters that can potentially overcome the effect of residual laser-frequency jitter. Indeed, the optical resonances manifest as a comb of sharp transmission features whose width $(\Delta v)$ and spacing $\left(v_{\mathrm{f}}\right)$ are typically in the ranges of $\mathrm{kHz}$ and $100 \mathrm{MHz}$, respectively. For instance, an optical cavity comprising two mirrors of reflectivity $R=0.99999$ separated by $1 \mathrm{~m}$, corresponds to $\tau=$ $333 \mu \mathrm{s}, \Delta v=477 \mathrm{~Hz}$ and an effective path length of $100 \mathrm{~km}$. This example illustrates the long photon lifetime, narrow mode width and extensive path length achievable with laboratoryscale high-finesse cavities.

Optical cavities are most commonly used to interrogate molecular absorption in two different ways. One can measure either the steady-state transmission of incident light through the cavity or the photon lifetime, both of which are sensitive to light absorption by the cavity medium. In both cases, the highest spectral resolution is achieved using mode-by-mode cavity excitation to yield spectra sampled at well-defined frequency intervals. The first approach leads to cavity-enhanced absorption spectroscopy (CEAS, Sec. 2.2) and the second to cavity ring-down spectroscopy (CRDS, Sec. 2.3). Importantly, unlike CEAS, CRDS is immune to intensity noise in the light source and to absorption outside the cavity. There are advanced versions of CRDS in which the resonant frequencies are made immune to laser-frequency drift and/or external perturbations via active cavity or laser stabilization methods. These include the frequency-stabilized CRDS (FS-CRDS) technique [3,4] and optical feedback frequency-stabilized CRDS (OFFS-CRDS) [5,6]. Both approaches are insensitive to laserintensity and -frequency noises, and are based on precise and accurate observations of modefrequency intervals ( $x$-axis) and time ( $y$-axis). They can provide line profiles with minimal instrumental distortion and SNRs routinely ranging from $10^{3}$ to $2 \times 10^{4}$ [7-10] with exceptional cases in the $2 \times 10^{5}$ to $10^{6}$ range $[5,11,12]$. Other cavity-enhanced methods include (Sec. 2.5 ) cavity mode-width spectroscopy (CMWS) which measures absorption-dependent widths of individual cavity resonances, and one-dimensional cavity-mode dispersion spectroscopy (1DCMDS) [13] which measures frequency shifts of the cavity resonances to quantify gasinduced dispersion. We note that CMWS is a high-resolution variant of CEAS that uses the mode width instead of the amplitude decrease to measure absorption and thus overcomes the need for spectrally broadband intensity normalization. The 1D-CMDS also shares this property and yields spectra where both axes are based upon measurements of the optical frequency.

Interestingly, optical cavities also can be simultaneously probed at several wavelengths in a multiplexed manner. For this, femto-second laser sources or electro-optic modulated CW lasers that consist of combs of phase-locked narrow emitters [14] can be efficiently coupled to an optical cavity. This can be achieved in a raw (cavity-enhanced optical frequency comb spectroscopy) [15-19], one-by-one (Vernier effect) [20,21], or in self- or multi-heterodyne 
configurations [22] to yield both field amplitude and phase information quantifying complexvalued line shapes.

In the remainder of Sec. 2.1 we provide a general description of light-matter interaction in an enhancement cavity. For simplicity, we consider a FP interferometer having two identical dielectric (absorption-free) mirrors with reflectivity $R$, and transmission $T$ such that $R=1-T$. Neglecting intracavity-medium scattering or other losses, considering a single transverse mode and in the limit of a linear absorption process, the complex-valued transmittance $\tilde{y}(v)$ of the incident field at frequency $v$ is given by the Airy formula:

$$
\tilde{y}(v)=\frac{T \exp [-(\alpha l+\mathrm{i} \Delta \phi) / 2]}{1-R_{\mathrm{e}} \exp (-\mathrm{i} \Delta \phi)},
$$

where all variables (except for $l$ ) on the right-hand-side depend upon $v$. $\alpha$ is the intensitybased absorption coefficient, $l$ is the resonator length, $\Delta \phi=2 \pi v t_{\mathrm{r}}$ is the phase shift corresponding to the round-trip time $t_{\mathrm{r}}=2 l / c$ (with $c$ the speed of light), and $R_{\mathrm{e}}=\mathrm{R} \exp (-\alpha l)$ is the effective mirror reflectivity that incorporates the single-pass absorption loss of the medium. The resonant frequencies occur when $\Delta \phi$ is a multiple of $2 \pi$. In the absence of absorption and dispersion effects, the modulus squared of the Airy formula reduces to a comb of equidistant peaks of unity transmission separated by the free spectral range (FSR) denoted by $v_{\mathrm{f}}$. Absorption reduces the peak transmission and broadens the resonances, whereas dispersion in the medium shifts the positions of the resonances relative to the empty-cavity case ( $c f$ Sec. 2.5 ).

With the incorporation of models for absorption and dispersion, the preceding formulas can be used to calculate the cavity transmission (amplitude and phase) by summing over all relevant electromagnetic field components at one or more frequencies. Both heterodyne and intensity-based transmission measurements can be modeled. In the former case, the field transmitted by the cavity is combined with that of a local oscillator (generally either a singleor multi-frequency laser such as an OFC), which results in a complex-valued transmission signal at one or more heterodyne beat frequencies. Similarly, for intensity-based measurements at a given frequency, the real-valued transmission is obtained by squaring the modulus of Eq. (1).

Note that the complex Airy formula, which is a comb-like function of $\Delta \phi$ representing a series of resonances, can be reduced to a complex Lorentzian profile near an individual resonance. This is a good approximation when the cavity finesse $F$, given by $\pi \sqrt{R_{\mathrm{e}}} /\left(1-R_{\mathrm{e}}\right)$, is large and when $\Delta \phi<<1$. In this limit, we can write the complex field transmission for excitation of a single cavity mode as:

$$
\tilde{y}(v)=\frac{T}{1-R_{\mathrm{e}}} \times \frac{\exp (-\mathrm{i} \Delta \phi / 2)}{1-\mathrm{i}(F / \pi) \Delta \phi},
$$

where $\Delta \phi=(2 \pi \Delta v) / v_{\mathrm{f}}$ and $\Delta v$ is the frequency detuning of the light source about the local resonance. Furthermore, for measurements of photon lifetime of the resonator ( $e g, \mathrm{CRDS}, \mathrm{Sec}$. $2.3)$, the time-dependent ring-down decay signal $s(t)$ is found by squaring the modulus of the Fourier transform of Eq. (2) which gives:

$$
\mathrm{s}(t)=s_{0} \exp (-t / \tau)
$$

with a frequency-dependent time constant

$$
\tau(v)=\frac{l}{c[1-R \exp (-\alpha l)]} .
$$


These results illustrate the simple correspondence between the frequency- and time-domain responses for excitation of a single cavity mode. Assuming high mirror reflectivity $(R \sim 1)$ and weak intra-cavity absorption ( $\alpha l<<1)$, Eq. (4) reduces to $1 /[c \tau(v)]=\alpha(v)+1 /\left[c \tau_{0}(v)\right]$, where $\tau_{0}(v)=l /[c(1-R)]$ is the time constant in the absence of absorption. This relation indicates that the $x$ and $y$ axes of CRDS absorption spectra can be determined from measurements of frequency and time, respectively, without explicit determination of path length. The power transmitted by the cavity, $P_{\mathrm{t}}(v)$ as detected by a photodiode placed after the resonator and assuming perfect mode matching, is given by the square of the modulus of Eq. (1). Assuming on-resonant excitation $(\Delta \phi=0)$, one has:

$$
P_{\mathrm{t}}(v)=P_{0} \frac{T^{2}}{[1-\operatorname{Rexp}(-\alpha l)]^{2}} \exp (-\alpha l),
$$

where all variables on the right-hand-side depend upon $v$ and $P_{0}$ is the incident power. For high mirror reflectivity and weak intra-cavity absorption, Eq. (5) can be expanded to first order in $\alpha l$, leading to:

with

$$
P_{t}(v)=\left(1-\alpha l_{\text {eff }}\right) P_{0}
$$

$$
l_{\text {eff }}=2 l /(1-R) .
$$

Eq. (7) explicitly highlights the path length enhancement induced by the resonant cavity as mirror reflectivity and cavity finesse increase. This enhancement effect is referred to in the cavity-enhanced absorption spectroscopy (CEAS, Sec. 2.2) acronym.

Although the first mention of the use of a FP assembly to enhance intra-cavity absorption is by Jackson [23], O'Keefe and Deacon [24] were the first to use CRDS ( $c f$ Sec. 2.3) to measure molecular absorption spectra. In general, the reader may refer to Chapt. 1 of [25] for a deeper understanding of the CEAS process and to [26] for CRDS.

Except for Secs. 2.9 and 2.10, the next sub-sections describe recent experimental efforts toward accurate spectroscopy, essentially disregarding low-resolution or large-apparatusfunction setups. We consider $\mathrm{CW}$ laser-based techniques with cavities to enhance sensitivity and/or resolution, which measure the cavity transmission (Sec. 2.2), the lifetime of photons trapped in the cavity (Sec. 2.3) or the cavity mode width (Sec. 2.5). The referencing of probe laser frequencies to an OFC for absolute calibration is also presented (Sec. 2.4). Laser-comb sources, which can be used with (Sec. 2.7) or without an enhancement cavity (Sec. 2.6), offering broadband coverage and/or multiplexed approaches also are discussed. Then, we evaluate (Sec. 2.8) the performance of dual-laser direct-absorption methods for high-precision line shape studies. Finally, the interests of Fourier transform spectroscopy and terahertz techniques for spectral-shape studies are discussed in Secs. 2.9 and 2.10, respectively.

Before discussing these various techniques, it is important to recall that obtaining spectra of very high quality is not the only criterion for accurate spectroscopic studies of pressure effects. Indeed, the reliability of the tests of theories and models as well as that of the spectral-shape parameters retrieved from fits of measurements also depend on the knowledge of the sample (composition, pressure, temperature, path length). Obviously, uncertainties on these experimental parameters can spoil many of the efforts made to improve the set-up and the quality of the data that it produces. While this rather technical and difficult issue is beyond the scope of this review, some discussions concerning the determination of the temperature can be found in some of the references cited in Sec. 5.2.1, the importance of knowing the gas composition being addressed in Sec. 5.2.2.

\subsection{Cavity Enhanced Absorption Spectroscopy (CEAS)}


An intuitive way to take advantage of the absorption-enhancement effect of a FP cavity consists in monitoring the frequency dependence of its on-resonance transmission as expressed in Eq. (5). This provides (within the limit of weak absorption) direct access to the absorption coefficient $\alpha$ through a Beer-Lambert-like law that exhibits an enhanced path length [Eq. (7)]. Note that, for studies of narrow absorption lines and accurate determinations of their areas, the base length $l$ and mirror properties must be accurately known because they govern the mode spacing and effective path length. Actually, the FP cavity length differs from a basic ruler-based measurement according to Gaussian mode theory [27,28] and may be determined from measurements of the local FSR ( $c f$ Sec. 2.5). The mirror reflectivity can be determined from a CRDS measurement (Sec. 2.3) or by a cavity-mode width measurement ( $c f$ Sec. 2.5). To address the problem of measuring absorption continua (Sec. 4.3), the mirror- and gas-dependent losses must also be independently characterized. Let us emphasize that the cavity mirrors' properties are generally frequency dependent because of the present coating technology (dielectric films), but also because of the refractive nature of the intra-cavity probed medium. From [29], the magnitude of the absorption-induced mode shift is $\sim 0.3 c \alpha(v)$. Referring again to a one-meter long FP cavity with $99.999 \%$ reflectivity mirrors (Sec. 2.1), a local absorption coefficient of $3.4 \times 10^{-7} \mathrm{~cm}^{-1}$ is sufficient to shift an FP resonance by its mode width. Furthermore, at wavelength of $1.6 \mu \mathrm{m}$ for instance, a $1 \mathrm{~nm}$ variation of the cavity length $\left(10^{-9}\right.$ relative) shifts all the FP resonances by about $200 \mathrm{kHz}$, which is more than 100 times the mode width. The use of high reflectivity mirrors therefore requires stable FP assemblies. On the other hand, typical tunable semiconductor lasers (eg telecommunications type) have line widths of the order of $1 \mathrm{MHz}$ (jitter), obviously leading to extremely noisy cavity transmission under $\mathrm{CW}$ excitation. However, the optical cavity and laser noise sources are of different physical origin and act on different time scales. An optical cavity, as any mechanical assembly, exhibits a slow thermo-mechanical expansion $(\sim 1 \mathrm{~s}$ scale $)$ that sums with acoustic noise $(<20 \mathrm{kHz})$ yielding time constants ranging from $\mathrm{s}$ to $\mathrm{ms}$ timescales, respectively. This makes it rather easy to control the cavity length, typically with piezoelectric transducers. Telecommunications semicondúctor lasers typically exhibit white noise that extends over several $\mathrm{MHz}$, making it difficult, but possible [30-32], to actively cancel the frequency noise on time scales comparable to the buildup time of the optical cavity. Note that external cavity diode lasers (ECDL) have narrower short-term line widths $(<500 \mathrm{kHz})$ than semiconductor lasers and are therefore better suited for electronic control loops that directly actuate laser current [33].

Several strategies have been developed to overcome highly intermittent laser-to-cavitylongitudinal mode overlap which occurs when the probe laser frequency and cavity resonance are not coupled. These methods include: (i) averaging a series of passages through resonance to reduce the intensity noise, known as Integrated Cavity Output Spectroscopy (ICOS) [34]. (ii) Off-axis injection of a FP cavity (OA-ICOS) [35] to induce the excitation of a series of higher-order transverse modes having various resonant frequencies. With an appropriate FP geometry, this results in quasi-continuous transmission, smoothing out the peaks of the Airy formula. Unfortunately, these two strategies are still limited in terms of SNR, essentially because of residual optical fringes, transverse-mode-dependent mirror losses and laser phase noise which manifests itself as fluctuations in the transmitted power. They are, therefore, not recommended for accurate line-profile determinations. (iii) Electronic or optical locking of the laser emission of an ECDL to a stable FP or other frequency reference is an efficient CEAS approach for accurate line-profile determination (Sec. 2.5). Several methods can serve this purpose, eg: PDH [36], tilt-lock [37] and NICE-OHMS [38]. An alternative attractive method consists in optical-feedback locking (OF-CEAS) [39]. Under certain conditions, the laser self-locks to the mode of the FP to which it is injected and remains locked even if one tries to detune it. This behavior results from counter-propagating cavity photons that seed the 
laser with the proper optical phase. Such a condition is achieved by precise control of the laser-to-FP distance, which typically requires a piezoelectrically actuated steering mirror and simple electronics. A drastic reduction of the laser emission line width occurs, well below the FP mode width, leading to near-perfect mode injections and allowing the laser frequency to be hopped over successive longitudinal modes of the FP. This technique can be applied to virtually any single-frequency semiconductor laser including DFBLs [40], ECDLs [41], QCLs [42] and ICLs [43].

For CEAS, the lowest limit of detection $\left(1 \times 10^{-14} \mathrm{~cm}^{-1}\right.$ for $1 \mathrm{~s}$ of averaging) was obtained using the NICE-OHMS method [38]. It was calculated from the noise level observed in a 1 MHz-wide saturation-dip structure having a peak absorbance of $4 \times 10^{-9}$ and SNR equal 7700 . For a broadband OF-CEAS spectrum spanning $30 \mathrm{GHz}, 8 \times 10^{-11} \mathrm{~cm}^{-1}$ was obtained based on the noise in the spectrum baseline [44].

\subsection{Cavity Ring-Down Spectroscopy (CRDS)}

In CRDS, the ring-down events [Eq. (3)] are insensitive to laser intensity fluctuation, enabling extremely high SNR spectra [6,11,12] and low detection limits [45] to be attained. However, laser jitter and/or cavity length fluctuation impair precise laser frequency determination and photon coupling into the cavity. The former affects the frequency axis, while the latter limits the data throughput and sets the ultimate reachable sensitivity, governed by photon shot noise. Consequently, the implementation of frequency locking mechanisms is mandatory for the acquisition of high fidelity absorption profiles. The first CRDS effort (with CW probe lasers) towards this goal involved a dual-laser scheme [3]. The length of the CRDS cell was actively locked to a frequency-stabilized reference laser, while a probe laser was brought into resonance, mode by mode, resulting in spectra having a precise optical detuning axis. This technique, called Frequency-Stabilized CRDS (FS-CRDS) was used in many lineshape studies $(e g[4,8,12,46])$ and had sufficient spectral resolution to reveal MHz-wide saturation dips [46]. The acquisition rate of ring-down events was greatly improved by locking the laser to the cavity with PDH frequency locking schemes [47-50]. This approach was refined [51] using a dual-polarization configuration and EOM-based frequency shifting, leading to robust locking of the probe laser to the ring-down cavity with a $130 \mathrm{~Hz}$ relative line width, $5 \mathrm{kHz}$-level ring-down acquisition rates, minimal dead-time between cavity mode excitations and decay-time measurement precision better than 1 part in $10^{4}$. Alternatively, a dual-cavity approach was used [5] which transferred the stability of a reference cavity to the ring-down cavity. First, the source laser was locked to individual modes of the reference cavity by optical feedback [39], with capability for mode by mode scanning. This step stabilizes the probe laser frequency and renders its bandwidth narrow by comparison to that of the ring-down cavity mode. Second, continuous optical tuning is realized up to $20 \mathrm{GHz}$ using a single-sideband optical modulator, transforming the stabilized single-frequency light source into an arbitrarily tunable probe laser [52]. Third, the ring-down cavity is locked to the probe laser allowing CRDS spectra to be acquired with fully adjustable resolution and high SNR. This approach has been used for applications to line metrology $[5,6,53]$ and more recently to $\Delta^{17} \mathrm{O}$ isotopic ratio measurements in $\mathrm{CO}_{2}$ [54]. Here, $\Delta^{17} \mathrm{O}=\ln \left(\delta^{17} \mathrm{O}+1\right)-\lambda_{R} \ln \left(\delta^{18} \mathrm{O}+1\right)-\lambda_{R}$, where $\delta^{17} \mathrm{O}$ and $\delta^{18}$ are the usual " $\delta$ " values for relative isotopic abundance and $\lambda_{R}$ is the slope of a reference line $R$, which is used to quantify non-mass-dependent isotopic anomalies from an assumed mass-dependent fractionation law. An unprecedented precision of 10 partsper-million for $\Delta^{17} \mathrm{O}$ was demonstrated [54].

The quality of the spectra retrieved by CRDS makes active frequency stabilization techniques perfectly adapted for ultra-precise line-shape recordings of weakly absorbing lines 
with SNRs as high as $10^{6}[5,11,12]$. In all these studies, the source laser optical frequency was measured against or locked to one tooth of an OFC for an absolute frequency determination as explained in the next section.

\subsection{Frequency-comb assisted methods}

The highly coherent and broadband nature of OFCs makes these devices appealing as light sources for high resolution spectroscopic experiments that require rapid and wide spectral coverage with a well-defined frequency axis (cf Secs. 2.6 and 2.7). Typically, the OFC repetition rate and carrier-envelope offset frequency are stabilized relative to an absolute frequency standard ( $\mathrm{eg}, \mathrm{Rb}$ or $\mathrm{Cs}$ clock), thus providing a comb of known laser frequencies with stabilities at the sub-kHz level. However, unlike the extremely high stability of OFC frequencies, the intensity can vary substantially with time and from tooth-to-tooth, thus driving the limit of detection and often resulting in highly structured spectral baselines that alter the observed line shape. These effects can be reduced (but not completely eliminated) by signal averaging and intensity normalization.

Another important use consists in referencing a probe laser to one tooth of a frequency-stabilized OFC. An early example includes CRDS line-shape measurements of the $\mathrm{S}(0)$ and $\mathrm{S}(1)$ transitions of deuterium near $3 \mu \mathrm{m}$, where a Cs-clock-referenced OFC was used to provide absolute frequencies of the pump and signal beams in a difference-frequency generation probe laser [55]. These experiments yielded line positions and intensities with relative uncertainties of $6 \times 10^{-8}$ and $1 \%$, respectively. In several other line shape experiments [7,30,53,54,56-59], the OFC was used as an optical frequency reference for a singlefrequency probe laser or for measuring the mode spacing of an optical resonator [60]. The OFC-assisted technique yields a much more accurate and precise $(<100 \mathrm{kHz})$ frequency axis than conventional techniques using a wavelength meter (>1 MHz precision). Thus, this approach is especially attractive for measurements of absolute line positions and pressureshifting coefficients with levels of performance that are substantially improved by comparison to those achieved with traditional FTS instruments.

Another possibility consists in locking the probe laser to a tooth of the OFC that is slowly tuned by changing its repetition rate [30,61-63], for instance.

\subsection{Cavity mode-width and mode-dispersion spectroscopies}

Progress in phase-locking CW lasers to high-finesse cavities has recently enabled the development of cavity-enhanced spectroscopic techniques based on high-precision measurements of the cavity mode widths and positions. As derived above, the exponentially decaying intensity measured in CRDS [Eqs. (3),(4)] is the time-domain equivalent of the cavity-mode width measured under $\mathrm{CW}$ excitation in the frequency domain, see Fig. 1. Specifically, the FWHM of a Lorentzian FP mode is given by $(2 \pi \tau)^{-1}$. This property is exploited in CMWS [64-66] where the absorption spectrum is retrieved from the widths of the cavity modes measured in transmission. Because the spectral interval probed is so narrow, this measurement is also relatively insensitive to the frequency-dependence of the probe laser intensity. The properties of CMWS make it complementary to CRDS (Sec. 2.3) in terms of achievable accuracies at various levels of intra-cavity absorption. For low absorption, where the ring-down time is long and the modes are narrow, a higher precision is expected with CRDS. For high absorption, the ring-down time is short while the modes are broad and the precision of CMWS tends to be higher. Considered together, the CRDS and CMWS methods provide a wider dynamic range of measurable absorption levels than those associated with either method considered alone. It is important to recall that, in the context of line-shape measurements, short ring-down times associated with strong absorption may lead to systematic errors caused by the finite bandwidth of the detection system. Therefore, CMWS 
potentially has a higher dynamic range than CRDS provided that the frequency axis of the cavity-mode spectrum is sufficiently accurate, as indicated in [64] and demonstrated in [67]. Experimental setups for CMWS require ultra-narrow probe laser line widths and sub-kHz resolutions of the frequency tuning to measure the $\mathrm{kHz}$-level mode widths of high-finesse cavities. This can be achieved in a dual-beam configuration, where two orthogonal polarizations of the laser beam are used: the first for phase-locking the laser to the cavity mode and the second for scanning through the neighboring cavity-mode shapes $[51,65,66]$. This technique also eliminates the influence of the cavity acoustic noise on the mode spectrum.
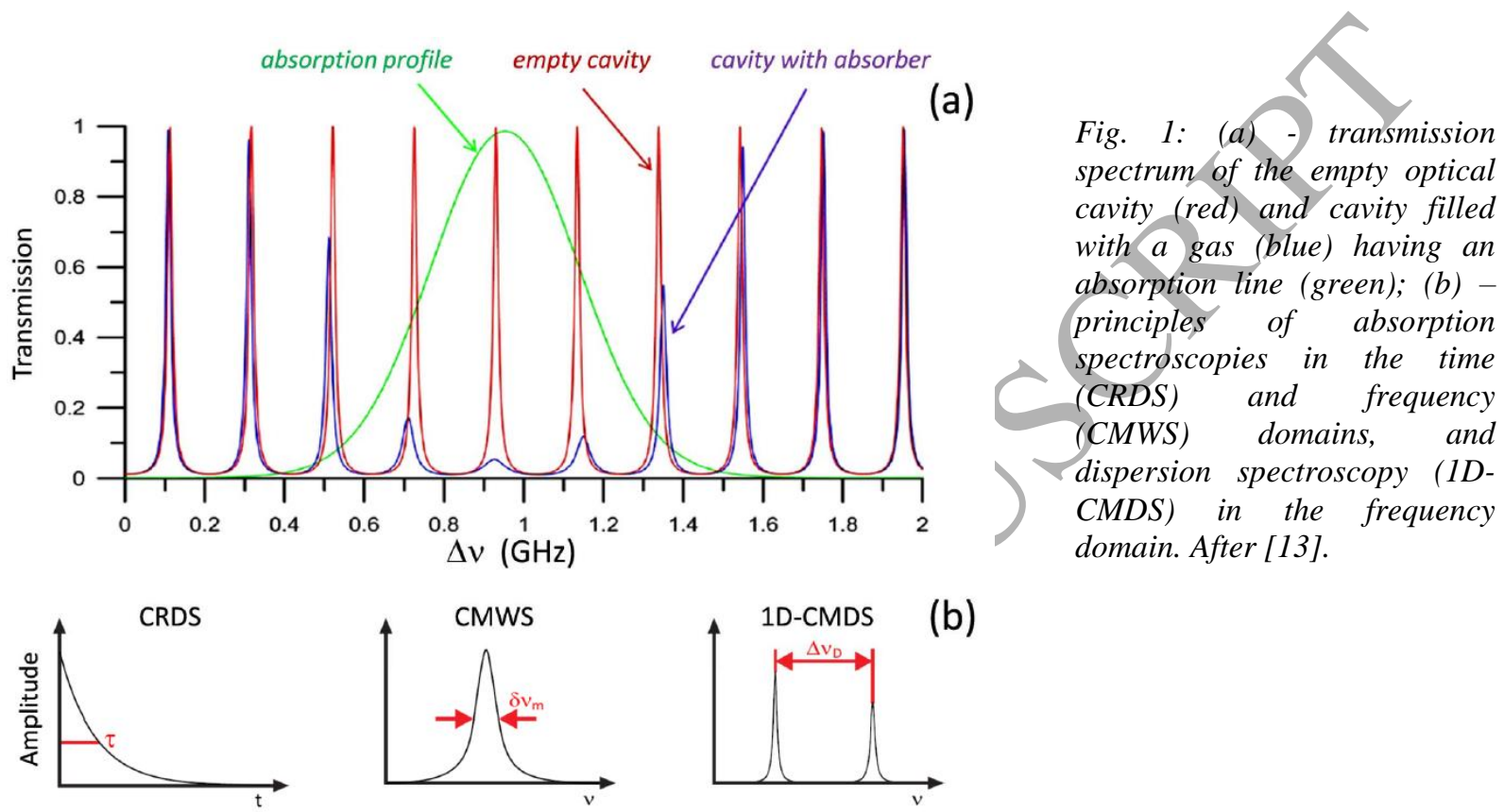

(b)

The measurement of dispersive mode shifts provides another interesting approach for quantitative spectroscopy because frequency is the physical observable that can be measured with the highest precision and lowest uncertainty. Furthermore, the determination of absolute or relative mode frequencies in the One-Dimensional Cavity Mode Dispersion Spectroscopy (1D-CMDS) [13] eliminates potential systematic errors in measured line-shapes caused by nonlinearities of the detection system. Contrary to "usual" spectra, which have absorption and frequency axes, in 1D-CMDS both axes of the dispersion or differential-dispersion spectrum are created from the same measurements of mode-center frequencies. They can thus be directly linked to primary frequency standards, as demonstrated in [67].

Comparisons of spectra obtained with different cavity-enhanced techniques also enable the quantification of potential systematic instrumental errors at the sub-percent level of uncertainty. For example, simultaneous measurements of both absorption and dispersion spectra provide a precise method to check the self-consistency of experimental line shapes. In [68], the accuracy of FS-CRDS (in the limit of strong absorption) was studied by comparison with 1D-CMDS. The line-shape fits revealed sub-percent level inconsistency between absorption and dispersion for high absorption, which could not be detected and were not manifest in the residuals of the individual fits, see Fig. 2. The above-described frequencybased cavity-enhanced techniques are thus particularly useful in applications ( $\mathrm{eg}$ studies of self-broadened line-shapes over a wide pressure range) that require highly accurate measurements spanning a broad range of absorption. For applications that require extremely high accuracy and SI traceability of measurements (eg Sec. 5.2), the frequency-based 1DCMDS should be an attractive alternative to traditional absorption techniques. So far both CMWS and 1D-CMDS were used for measurements of the shapes and absolute positions of 
CO lines with SNRs exceeding $10^{4}$ [67]. CMWS was also applied to low-pressure CO spectra [69] using a NICE-OHMS experimental setup. It was recently demonstrated [70,71] that the CMWS and 1D-CMDS methods also can be combined with optical frequency comb singletooth resolved spectroscopy [72]. In this case a broadband frequency-based absorption and dispersion spectrum can be measured with a kHz-level resolution ( $c f$ Sec. 2.7).

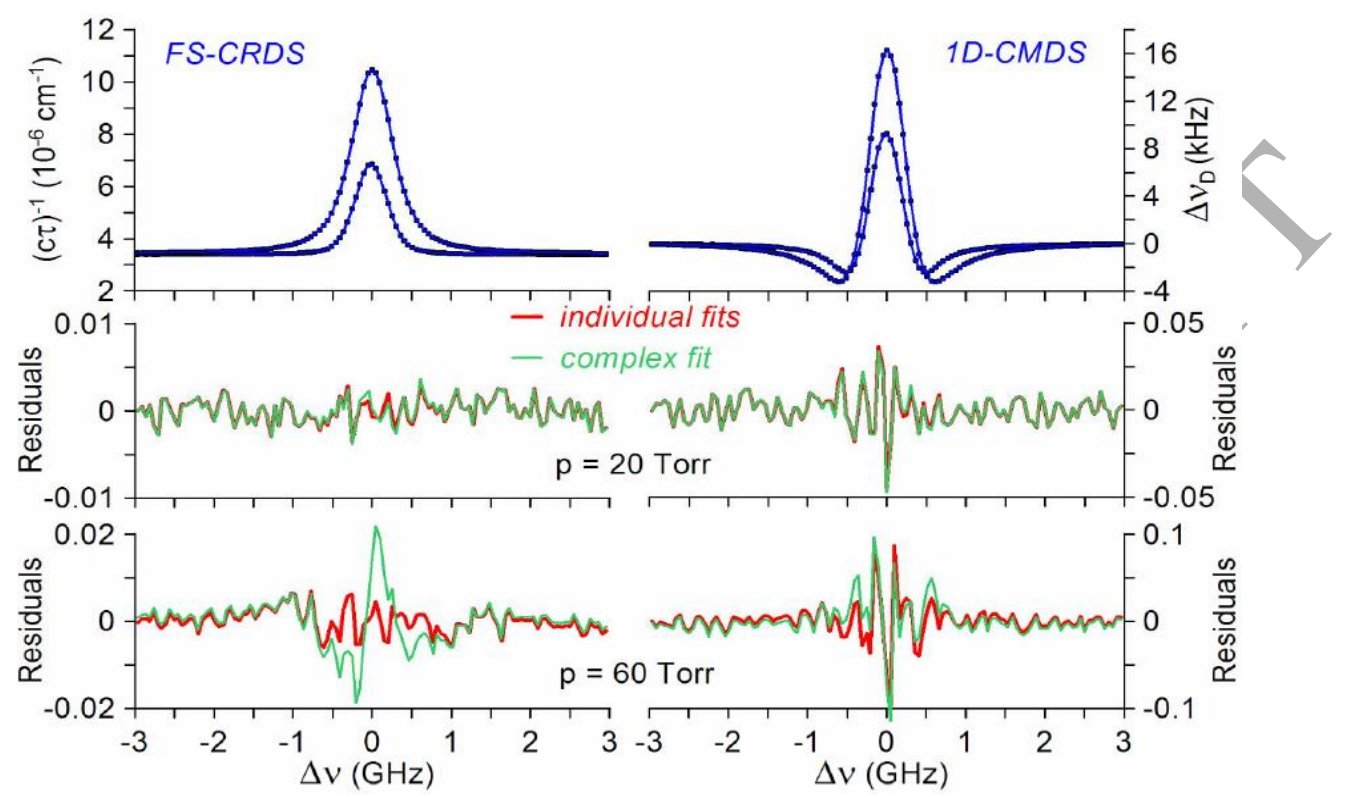

Fig. 2: Complex spectra (absorption from FS-CRDS and differential dispersion from $1 D-C M D S)$ of ${ }^{13} C^{16} O(3-$ $0)$ band P3 line measured at two CO pressures p. Below residuals from the complex speed-dependent NelkinGhatak profile fits (red) reveal perfect agreement between FS-CRDS and ID-CMDS at $p=20$ Torr and considerable disagreement at $p=60$ Torr. Residuals from the individual absorption or dispersion fits (blue) do not allow detection of this systematic line-shape distortion. After [73].

\subsection{Direct frequency-comb spectroscopy}

For OFCs, the extremely high stability of the frequency axis combined with their broadband spectrum makes them attractive light sources for absorption measurements. A review of various experimental schemes of direct OFC-based spectroscopy can be found in [74]. For line-shape investigations, single-tooth resolved methods, which provide a spectral resolution limited only by the OFC stability, are particularly attractive. Indeed, FTS combined with an OFC source is suitable to line-shape studies since it combines extremely high resolution with a broad spectral coverage. Two approaches to this experimental scheme were developed: dual comb and mechanical FTS comb spectroscopy.

- Dual Comb with two femto-second lasers: The idea of Dual Comb Spectroscopy (DCS) [75] is to interfere two OFCs having slightly different repetition rates, see Fig. 3. When both OFCs are combined and transmitted through a gas sample, the down-converted heterodyne beat spectrum of slightly detuned comb teeth provides an intensity absorption spectrum, similar to traditional FTS [76,77]. If only one OFC passes through the sample and the other serves as a local oscillator [78,79], both the absorption (amplitude) and dispersion (phase) spectra of the sample can be measured, analogous to dispersive FTS [80]. Note that for high SNR measurements using DCS, it is crucial to normalize the spectra for frequency- and timedependent variations in both OFC amplitudes $[79,81]$. Coherent signal averaging also requires that the relative line width between corresponding teeth of the two combs is not larger than the inverse of the averaging time, which in practice requires phase-locking of both combs to the same optical reference [81]. Several studies of molecular spectra with DCS have demonstrated its potential for line-shape investigations. For instance, absorption and 
dispersion spectra of HCN with SNRs up to 4000 were measured [81]. DCS was also used to detect variations in atmospheric $\mathrm{CO}_{2}, \mathrm{CH}_{4}$ and $\mathrm{H}_{2} \mathrm{O}$ [82]. In this case, the high-resolution spectra enabled comparison of the fit results obtained using the VP and SDVP with linemixing for $\mathrm{CO}_{2}$ lines. Another example is given by [83] in which the positions of $\mathrm{CH}_{4}$ lines in the $v_{3}$ band were accurately determined from absorption and dispersion spectra having SNRs up to 3500 and a spectral point spacing 4 times smaller than the OFC repetition frequency.

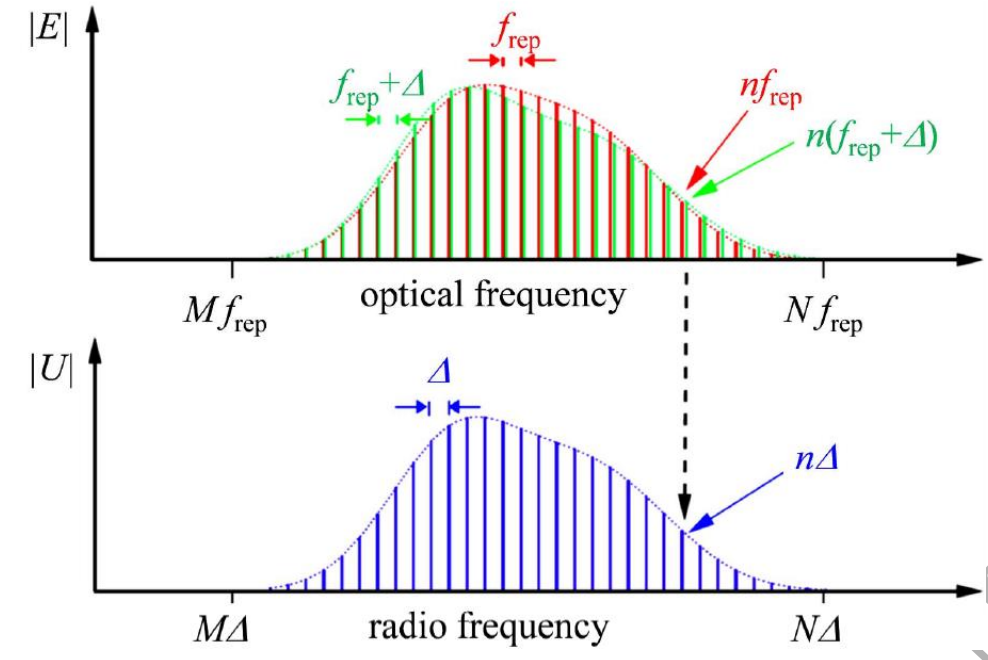

Fig. 3: Principle of the DCS using multiheterodyne detection. Interference between two slightly detuned OFCs (having repetition frequencies $f_{\text {rep }}$ and $\left.f_{\text {rep }}+\Delta\right)$ creates a new comb downconverted to $R F$, with frequencies equal to the differences between the optical comb teeth frequencies (nA). This new comb contains information on amplitude and phase change caused by molecular absorption of one OFC. After [77].

- Dual Comb with mini-combs: An alternative approach to DCS uses frequency combs generated from a single continuous-wave laser radiation [14] by two electro-optic phase modulators with slightly different modulation frequencies. Such combs have a high relative coherence and enable over 2 hours of real-time signal averaging. The achieved $50 \mathrm{GHz}$ span of the comb spectrum in the near infrared enabled simultaneous measurement of $\mathrm{CO}_{2}, \mathrm{CO}$, $\mathrm{H}_{2} \mathrm{O}$ and $\mathrm{HDO}$ lines at atmospheric pressure [84]. When compared to the femto-second OFCs, this system has a low cost and easily adjustable comb spacing but a relatively narrow bandwidth. It can potentially provide higher SNR due to the extremely high coherence of the two combs and high power per tooth. These features make it suitable for accurate line-shape measurements.

- Comb-based FTS with sub-nominal resolution: As recently shown [72], Fourier-transform comb spectroscopy based on a single OFC and a Michelson interferometer can deliver the resolution of a comb-tooth, surpassing the natural limits of traditional FTS because of the unique properties of the OFC. When the travel length $\Delta$ of the Michelson interferometer moving mirror equals $c / f_{\text {rep, }}$, where $f_{\text {rep }}$ is the repetition frequency of the comb, crosstalk between frequency elements (the comb teeth) after Fourier transformation is completely eliminated (Fig. 4). Any spectral points between the comb teeth can be probed in consecutive scans with different $f_{\text {rep }}$ and $\Delta$. This technique enables the construction of relatively low-cost and compact FT spectrometers with a resolution limited only by the comb line width. Proof-of principle line-shape measurements were made in the fundamental band of $\mathrm{CO}$ and $3 v_{1}+v_{3}$ band of $\mathrm{CO}_{2}$ with SNR of several hundred [72]. In the latter case, a high-finesse cavity locked to the comb was used to enhance the path length. This method was used recently to study nonVoigt spectral shapes of $\mathrm{CO}_{2}$ lines [85]. With a SNR of about 250 the effects of the speed dependence of the broadening were clearly observed. 


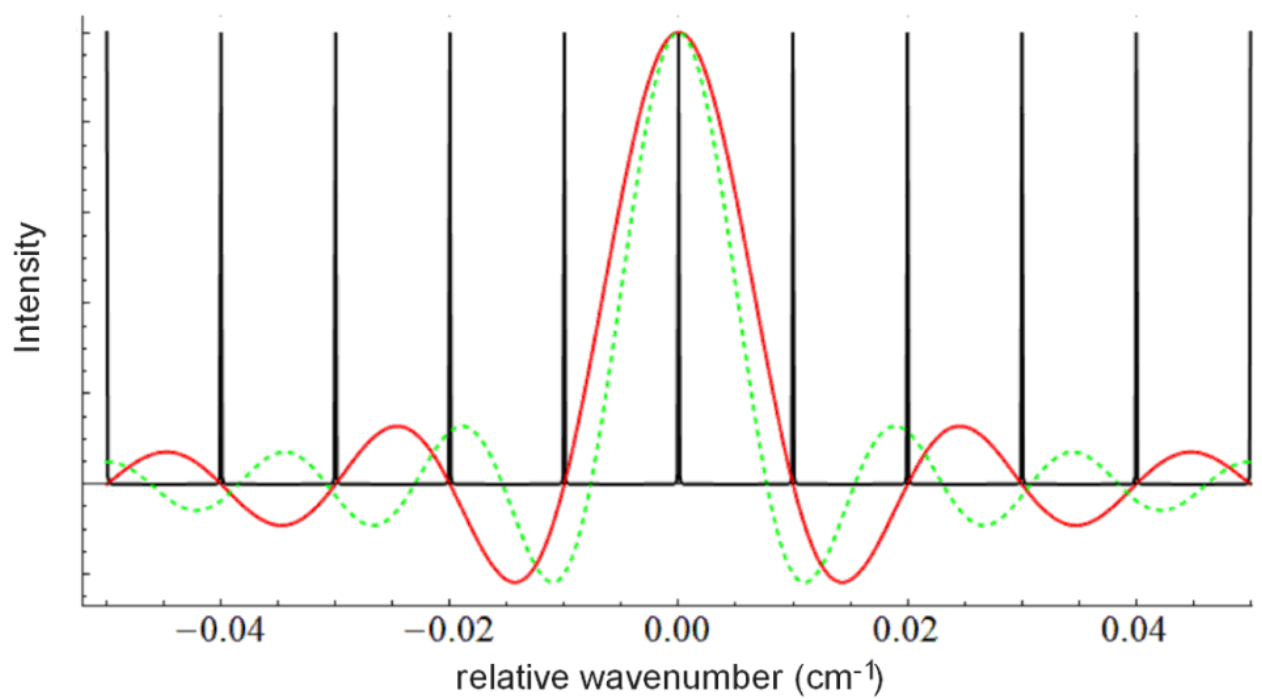

Fig. 4: Principle of the comb-based FT spectroscopy with sub-nominal resolution. When the length of the FT interferogram $\Delta$ equals $c / f_{\text {rep }}$ the instrumental sinc function (red curve) of given transformed comb tooth crosses zero at multiples of frep which correspond to consecutive comb teeth (black curve). In this case FT instrumental function does not influence the measured spectrum. When $\Delta$ differs from c/frep, the corresponding sinc function (green dashed curve) has non-zero values at the comb teeth frequencies and influences the spectrum. After [72].

\subsection{Cavity-enhanced direct frequency-comb spectroscopy}

The use of a high-finesse cavity to improve the sensitivity of DCS is attractive. However, mirror- and gas-induced dispersions in the cavity affect the cavity-mode spacing and preclude the perfect matching with the equally spaced teeth of the OFC over a broad spectral range. Because this frequency mismatch affects the transmission spectrum, it must be properly modeled [86] so that dispersion must be accounted for when tightly locking a highfinesse enhancement cavity to a broadband OFC [87]. The problem may be also solved, at the cost of lower transmitted power and effective enhancement, using a dither locking scheme [15-18] in which the cavity length oscillates around the resonance with the comb and the oscillation amplitude is larger than the dispersive mode shifts.

Direct frequency comb based Fourier-transform spectroscopy methods described in the previous section were also used with enhancement cavities. A Michelson interferometer based FT was used to record $\mathrm{CO}_{2}$ line shapes with SNRs of several hundred [72] and cavityenhanced DCS for high-resolution spectroscopy was demonstrated in [14].

In comb-based Vernier spectroscopy $[20,88]$ the cavity is used to filter the comb modes by setting the cavity FSR to a value slightly different than the comb-teeth spacing $f_{\text {rep }}$. Such a filtered comb contains a reduced number of teeth with increased distance between them. Every $n^{\text {th }}$ tooth is transmitted through the cavity while all others are reflected. Here $n$ must be large enough to enable single-tooth resolution by a dispersive grating or VIPA etalon. Their transmission may be measured simultaneously by a detector array, eg CCD. By precisely tuning the cavity length, an arbitrary set of filtered comb teeth can be selected in consecutive measurements. This method is competitive with the previously described direct FT-comb spectroscopies. This approach provides equally high resolution and a higher powerper-spectral element, but lower spectral coverage of the parallel measurement. For quantitative absorption measurements, one must account for the mismatch between the cavity modes and the comb teeth caused by broadband and resonant dispersion in the cavity [86]. Examples of spectra with individual line shapes having SNR over 100 were presented for CO [86] and HCN [89]. In the latter case, both absorption and dispersion spectra were recorded.

Broadband CMWS and 1D-CMDS measurements (Sec. 2.5) with an OFC as a light source were recently demonstrated. This experimental scheme significantly increases the 
acquisition rate of broadband spectra (by comparison to $\mathrm{CW}$-laser setups) by the parallel measurement of hundreds of the cavity mode widths and shifts. In these methods of absorption and dispersion spectroscopy achieving single comb tooth resolution is critical. Both subnominal-resolution FT [71] and optical Vernier with a VIPA etalon [90] schemes yielded sub-kHz precision of the cavity mode and position measurements. The two approaches are based solely on frequency measurements and the recorded spectra therefore are insensitive to OFC power variations. Note that these methods have uncertainties that are unaffected by dispersion in the enhancement cavity [86]. They are thus promising for highprecision spectral line-shape measurements covering entire molecular bands.

\subsection{Dual-laser absorption spectroscopy}

Recording a line with high precision necessitates a stable and narrow optical frequency reference to which the probe laser can be referenced. Rather than using the tooth of an expensive self-referenced frequency comb (Sec. 2.4), an alternative solution was proposed [91] in which a kHz-linewidth-master laser (ML) is locked to a saturated absorption feature $[33,92]$. The probe laser is then forced to maintain a precise frequency-offset, provided by a radiofrequency $(\mathrm{RF})$ synthesizer which is phase locked to an ultra stable $\mathrm{Rb}$ oscillator, from the ML. By tuning the RF frequency, it is possible to perform continuous, highly linear and accurate frequency scans of the probe laser around a given eenter frequency [91]. This feature, in conjunction with the extremely high linearity of the detection electronics, makes it possible to avoid any instrumental distortion in measured absorption line shapes, reaching an experimental accuracy limited only by the shot noise.

This dual-laser method has been used to investigate the shape of $\mathrm{H}_{2}{ }^{18} \mathrm{O}$ lines in the Doppler regime [93], evidencing the influence of speed-dependences of the pressurebroadening and shifting coefficients. Subsequently, the quality of the quadratic and hypergeometric models for these speed dependences was tested [94] ( $c f$ Sec. 3.2.4c). A recent improvement of the dual-laser technique involves a reference laser and the NICE-OHMS approach, making it possible to reduce the uncertainty associated with the frequency scale and allowing locking on weaker reference lines [95]. This technique was applied to measurements of spectroscopic parameters of $\mathrm{C}_{2} \mathrm{H}_{2}$ leading, in particular, to a determination of the $2 v_{3}+v_{5}$ band $\mathrm{P}_{\mathrm{e}}(14)$ line strength with a combined uncertainty of $0.22 \%$ [96]. Apart from molecular spectroscopy, the dual-laser technique has been successfully applied to the fields of Doppler thermometry and isotope research, as described in Secs. 5.2.1 and 5.2.2.

Finally note that the technique of offset-frequency locking of a pair of diode lasers is extensively used in atomic physics experiments, while it has been almost ignored in the field of molecular spectroscopy, until the work described in [97], in which the authors propose a dual laser method for water-vapor differential absorption sensing ( $c f$ also [98]).

\subsection{Fourier-transform spectroscopy (FTS) methods}

With the exception of direct frequency-comb-based methods ( $c f$ Sec. 2.6), the spectral resolution of most FT spectrometers is generally lower than that of laser-based systems. Nevertheless, these instruments typically provide more global spectral-shape information than can be obtained using relatively narrowband laser methods. Key attributes of FTS include the applicability to optically thick samples and the realization of accurate frequency axes with spectral coverage that is sufficient to investigate entire absorption bands. These attributes of FTS are most effectively exploited by carrying out physically constrained, multispectrum analyses [99] of several measured spectra that are acquired at known values of sample composition, pressure and temperature. In the last decade and before, such methods have provided a wealth of measured spectra for the test of theories and models, as well as for the determination of absorption-shape parameters from their fits. Given their broad spectral 
coverage and high data throughput, they have been, and remain, the workhorse for populating line-by-line spectroscopic databases. Numerous references cited in Secs. 3 and 4 demonstrate the interest of FTS and, in many cases, its advantages. An example can be found in [100] where information on individual line shapes, on line mixing and on collision-induced absorption in the $\mathrm{O}_{2}$ A band has been simultaneously retrieved from FTS and CRDS spectra. This study shows the complementarity of these two experimental methods and the interest of investigating an extended spectral range that is analyzed through the simultaneous fit of spectra recorded at various pressures. The interest of such a method when the FTS is coupled to coolable cells ( $\mathrm{eg}$ [101]) is further illustrated by [102], where multispectrum fits of broadband $\mathrm{CH}_{4}$ spectra recorded for various pressures and temperatures enabled the retrieval of information on line-broadening, -shifting and -mixing coefficients together, for some lines, with the speed dependence. In addition, FTS has brought a lot of experimental information on the broadband water vapor continuum and on collision-induced absorption spectra, as shown by many references cited in Secs. 4.3.1 and 4.4. Let us finally mention a study [103], which is to our knowledge the first in which FT spectra have enabled the investigation of the effects of temperature on the parameters of a very refined individual line-shape model. Indeed, the $T$ dependences of not only the line-broadening and -mixing coefficients but also those of the speed dependence of the width and of the Dicke narrowing parameter have been determined from fits of measured spectra.

\subsection{Advances in coherent terahertz spectroscopy}

The measurement of light-matter interaction in the terahertz spectral region (roughly 0.1-10 THz) is a fertile research area with numerous applications in spectroscopy. Although many materials that are opaque or absorb at infrared and visible wavelengths are transparent for $\mathrm{THz}$ (also known as mm-wave), there are species, such as water vapor, which have strong rotational absorption fingerprints there. Given the relatively small Doppler widths of transitions in this region, the line shapes are dominated by collisional effects at relatively low pressure.

Coherent sources required for $\mathrm{THz}$ spectrometers can be produced by photomixing of infrared lasers, backward wave oscillators or by frequency multiplication of radiofrequency (RF) sources [104]. These types of sources typically provide output powers no greater than a few microwatts, which has hindered more widespread implementation of high-resolution spectroscopy in the $\mathrm{THz}$ region. Recently, compact quantum-cascade lasers (QCLs) are emerging as attractive sources with emission frequency down to $5 \mathrm{THz}$, output powers as high as $10 \mathrm{~mW}$ and line widths from $100 \mathrm{~Hz}$ to $500 \mathrm{kHz}$ [105-108]. The first high-resolution absorption spectroscopic measurement with a THz QCL was in 2006 and involved measurement of $\mathrm{CH}_{3} \mathrm{OH}$ [105]. More recently, a frequency-comb-assisted $\mathrm{THz}$ QCL spectrometer resulted in an SNR of approximately 3000 and line centers measured with an uncertainty of $10 \mathrm{kHz}$ [108]. These experiments clearly demonstrate the potential of QCL technology for realizing new high-precision line-shape studies in the $\mathrm{THz}$ region, although the stability of cryogenic detection schemes often remains a limiting factor in measurement precision.

Numerous $\mathrm{THz}$ line-shape studies were carried out prior to the development of $\mathrm{THz}$ QCL sources. A spectrometer employing a custom-built coherent radiation source (based on photo mixing of two near-infrared lasers) provided temperature-dependent collisional width and shift coefficients of water vapor [109]. These measurements were made over the temperature range 263-340 K, yielding a spectral resolution of $15 \mathrm{MHz}$ and relative uncertainty in the broadening coefficient from 1 to $3 \%$. In [110-112], a coherent source comprising a phase-locked, frequency-multiplied RF diode was developed to measure self-, nitrogen- and oxygen-broadened pure rotational lines of $\mathrm{HNO}_{3}$ and $\mathrm{H}_{2} \mathrm{O}$. Absorption spectra 
were acquired using intensity- and frequency-modulation methods with phase-sensitive detection, and various line shapes were fit to the data to yield pressure-broadening and shifting coefficients as well as speed-dependent broadening and collisional narrowing parameters. Recently, a coherent $\mathrm{THz}$ source based on frequency-multiplied RF synthesizer signals with a resolution of 5-10 MHz was used to measure absorption by water vapor within a multipass cell [113]. A global, multispectrum fit of 145 spectra yielded line positions, shift and broadening parameters as well as continuum coefficients for several line profiles.

An experiment using a phase-locked Gunn diode source [114] demonstrated the feasibility of CRDS measurements in the $75-100 \mathrm{GHz}$ region. Subsequently, high-resolution determinations of $\mathrm{N}_{2}$ and $\mathrm{O}_{2}$ continua and $\mathrm{H}_{2} \mathrm{O}$ rotational lines in the $\mathrm{THz}$ region were made using a fast-scanning (30 GHz/s) BWO source with a tuning range of $170-260 \mathrm{GHz}$ [115]. This experiment employed a 10-m-long cavity, with finesse of 1000 , line width of $13 \mathrm{kHz}$ and resulted in a spectrum baseline noise of $2 \times 10^{-7} \mathrm{~cm}^{-1}$. Also using a FP resonator, cavity line width measurements straddling the microwave and $\mathrm{THz}$ regions were used to measure $\mathrm{O}_{2}$ and $\mathrm{H}_{2} \mathrm{O}$ absorption line shapes, with baseline noise levels of $4 \times 10^{-9} \mathrm{~cm}^{-1}$ and spectrum SNRs approaching 1000. Recently, cavity-enhanced measurements of $\mathrm{N}_{2} \mathrm{O}$ near $0.1 \mathrm{THz}$ were realized with a low-mass, CMOS-based instrument having a 3-cm-long resonator, finesse of 200 and mode width of $20 \mathrm{MHz}$ [116]. Fourier transforming the echo induced by the pulsed source yielded pressure-broadened $\mathrm{N}_{2} \mathrm{O}$ line shapes nominally $1 \mathrm{MHz}$ wide and a detection limit of $5 \times 10^{11}$ molec. $\mathrm{cm}^{-3}$. It is worth noting that although cavity-enhancement in these long wavelength regions increases measurement sensitivity compared to direct absorption methods, the maximum finesse of metallic-reflector-based resonators is typically diffraction limited and nominally two orders of magnitude lower than that achieved with the super-polished dielectric mirrors available for visible and near-infrared systems.

\section{Theories, models, results}

\subsection{Introduction and selection criteria}

This section is devoted to theories, models and results concerning pressure effects on absorption spectra, which are to be understood as follows: "Theories" means theoretical approaches that enable direct predictions of some measured quantities (spectra, line widths, etc). Within this topic we limit ourselves to those proposed since [1], ie 2007. "Models" refers to those assumptions and resulting equations that enable the description of experimental results based on parameters that are fitted to measured data. Here again, we focus on those proposed in the last decade. "Results" must be understood in the sense of comparisons between the predictions of "theories" and experiments, or tests of the ability of "models" to represent measurements. We consider those results obtained since 2007, regardless of when the theory or model used was published.

The remainder of Sec. 3 is divided into five parts in which we try to update the information given in chapters III-VI of [1]. Section 3.2 focuses on "isolated lines" that are not affected by neighboring transitions but are influenced by various effects leading to non-Voigt profiles. The calculations of "pressure-broadening and -shifting coefficients", primary ingredients of their shapes, are discussed in Sec. 3.3. "Line-mixing", through which the transfer of population between rotational levels has spectral consequences not too far from line centers, is the subject of Sec. 3.4. Section 3.5 then focuses on the spectral regions of the "far wings and associated continua" to which many processes, including the finite duration of collisions, contribute in a complex manner. Finally, "collision-induced absorption", when the interaction of molecules with light results from the dipole that only exists during intermolecular collisions, is considered in Sec. 3.6.

Note that most of the studies discussed in this section have been made at or near room temperature. In the following, we will thus generally give information on the temperature- 
range investigated only when the latter is broad enough to enable a significant test of the ability of a theory or model to describe the $T$ dependence.

\subsection{Isolated line shapes}

As in Chapt. III of [1], we consider here "isolated transitions", ie those sufficiently distant from others for their core region not to be influenced by their neighbors under the considered gas conditions. Describing their shapes requires simultaneously accounting for the Doppler effect, the collision-induced velocity changes of the radiating molecule, and the pressure-induced speed-dependent line broadening and spectral shifting. With regard to this topic, the last decade has witnessed several advances, largely based on the high quality of spectra recorded with laser techniques (Sec. 2). Various phenomenological line-shape models have been proposed and/or tested, while theories for predictions of the line shape were developed. In the first part of this section, we present a new approach for the direct calculation of isolated line profiles (Sec. 3.2.1). Other approaches evaluating the spectral shapes by using a kinetic equation describing the time evolution of the dipole auto-correlation function (ACF) are presented in Sec. 3.2.2. Models and methods developed or used to compute the velocity collision kernels involved in this equation are also reviewed in Sec. 3.2.2. Approaches proposed that account for relativistic and dispersion effects on the line shape are the subjects of Sec. 3.2.3. Finally, recent advances concerning phenomenological line-profile models are reviewed in Sec 3.2.4.

\subsubsection{Direct and empirically corrected predictions from Molecular Dynamics}

\section{$\underline{\text { Simulations }}$}

A new theory for the direct prediction of isolated line shapes of linear molecules was proposed in [117]. It uses classical dynamics and an assumed intermolecular potential to compute the translational and rotational evolutions of a large number of molecules. These Classical Molecular Dynamics Simulations (CMDS) provide the center-of-mass position $\vec{q}_{m}(t)$ and velocity, as well as the axis orientation $\vec{u}_{m}(t)$ and rotational angular momentum, of each molecule $m$ at all times $t$. For the linear species considered, the dipole moment lies along the molecular axis and its ACF is proportional to the average $\langle\vec{u}(0) \cdot \vec{u}(t)\rangle$. The Doppler effect is introduced through the relative displacement $\left[\vec{q}_{m}(t)-\vec{q}_{m}(0)\right] \cdot \vec{z}$ along the radiationpropagation direction $\vec{z}$. The Laplace-Fourier transform of this ACF (ie multiplying the ACF by $\exp (-i \omega t)$ and integrating from $t=0$ to $+\infty)$ then yields the absorption spectrum. However, because CMDS lead to a continuous distribution of rotational speeds, this spectrum shows no line structure. In order to solve this problem, a requantization procedure was proposed [117]. As first demonstrated for pure $\mathrm{CO}_{2}[117,118]$ and confirmed for other systems $[119,120]$, these requantized CMDS (rCMDS) closely predict the observed relative deviations of line shapes from the VP without adjusting parameters. Note that an alternative requantization was proposed in [121], which is more suitable for light rotors and was successfully applied to $\mathrm{HCl}$ [121] and $\mathrm{CH}_{4}$ [122] lines. It also enables one to constrain the positions and intensities of the lines in the calculated spectrum to literature values (taken from a database, for instance), a possibility that was used in $[123,124]$.

While rCMDS accurately predict the relative deviations of line shapes from the best VP fits, the line shapes themselves are not as well described because the line widths in rCMDScalculated spectra may deviate from measured values by several percent ( $\mathrm{eg}[117,120,121])$. An approach to empirically correct for these discrepancies was proposed in [125] and successfully tested for some CO lines. The idea is to multiply the rCMDS-calculated dipole ACF for line $\ell$ at pressure $P$ by the correction factor $\exp \left(-2 \pi c \Delta \gamma_{\ell} P t\right)$. Here, $\Delta \gamma_{\ell}$ is the 
difference between the pressure-broadening coefficients obtained from fits, with the same line shape, of a measured spectrum of line $\ell$ at a single pressure $P_{0}$ and of that obtained from uncorrected rCMDS for the exact same conditions. With this simple correction, differences with experiments may become as small as a few times $0.1 \%$ of the peak absorption over a broad range of $P$. This is illustrated by Fig. 5 which also shows the large errors obtained when the VP is used. Note that the results in this figure are for room temperature and that the ability of the proposed approach to accurately represent spectra at various temperatures remains to be proven. However, since the model offers the possibility to introduce an ad hoc temperaturedependent correction $\Delta \gamma_{\ell}(T)$ we expect it to lead to results of similar quality below and above room temperature.

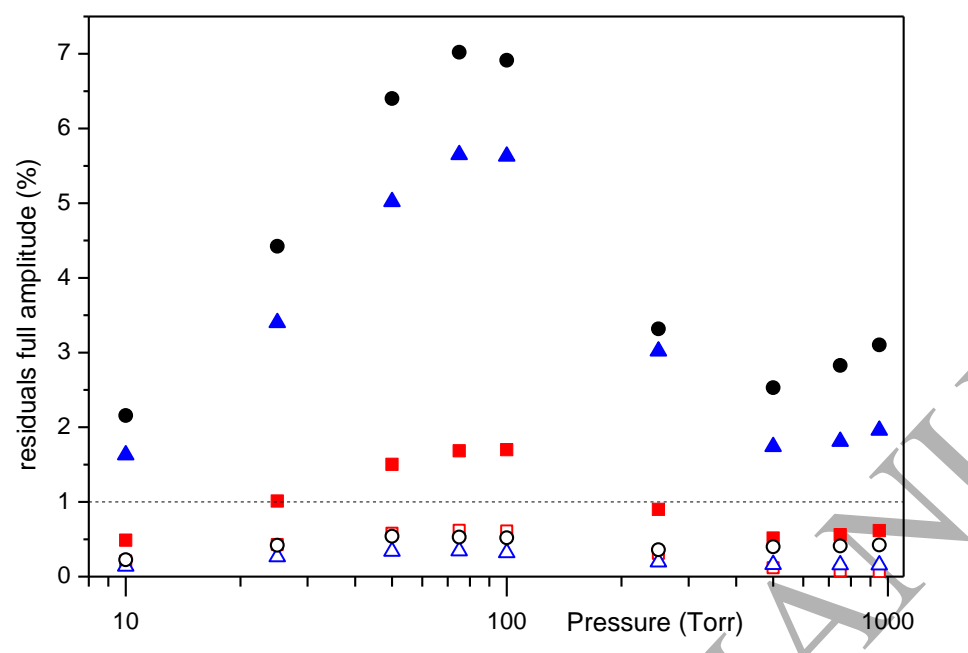

Fig. 5: Peak-to-peak amplitudes (relative to the absorption peak values) of the residuals of multispectrum Voigt-fits of measured spectra of the $P(17)$ line of the 3-0 band of $\mathrm{CO}$ at room temperature (full symbols) and relative deviations between measured and correctedrCMDS spectra (open symbols) for $\mathrm{CO}$ diluted in $\mathrm{He}$ (squares), $\mathrm{Ar}$ (triangles) and $\mathrm{Kr}$ (circles). After [125].

\subsubsection{Using a kinetic equation}

An alternative approach to direct rCMDS is to use a set of (vectorial) kinetic equations that drive the time evolution of the dipole ACF ([126-129] and Sec. II.5 of [1]). A general review on this topic can be found in [130]. In a purely theoretical study, using the $1 / r^{n}$ potential form, [131] solved these kinetic equations and showed the influence of the interaction potential (ie of $n$ ) on the calculated spectral shape. Within the "isolated-line approximation" (no transfer of intensity between the various transitions) this set of kinetic equations becomes diagonal in the line space with one independent differential equation for each transition. The latter involves, besides the Doppler contribution, a collision kernel for the changes of the radiator velocity together with a collisional width (and shift) that depends on the radiator speed. It is also possible to introduce [126] a parameter describing the correlation between the collision-induced changes of the molecular translational and rotational motions. While the speed-dependent pressure-broadening and -shifting coefficients can be obtained from calculations (Sec. 3.3), several approaches have been proposed for the velocity collision kernels. The latter have been modeled in recent studies by using the Keilson-Storer (Sec. 3.2.2a) and the billiard-ball (Sec. 3.2.2b) models, as well as directly calculated using CMDS (Sec. 3.2.2c). Note that, with the exception of the billiard-ball model which was tested (for $\mathrm{H}_{2}$ ) in a wide temperature range, all other recent results discussed in this section are limited to room temperature. This stresses the need for further tests of the models described below over extended temperature conditions.

\subsection{2a The Keilson-Storer model for the velocity collision kernel}

The Keilson-Storer (KS) model [132] was used for the first time in [133] to describe the effects of collision-induced velocity changes on line shapes (of $\mathrm{H}_{2}$ in $\mathrm{Ar}$ ). The KS kernels are based on two parameters [132,133]: The velocity-changing collision frequency $v_{\mathrm{VC}}$, and 
$\alpha$ which parameterizes the efficiency with which collisions change the velocity. The latter quantity takes on values between 0 and 1 , which correspond to two physical limits. For $\alpha=0$, there is no correlation between the velocities before and after collision. This is the hard collision model $[126,134]$ for which the molecular velocity is completely thermalized after each collision. For $\alpha=1$, the velocities before and after a collision are completely correlated and this is equivalent to the soft collision model [135]. The KS kernel is thus more general than these two extreme cases. Because collisions may change the velocity modulus and orientation with different efficiencies, a more general version was proposed [136] based on two distinct $\alpha_{\mathrm{m}}$ and $\alpha_{\mathrm{o}}$ parameters. In [137], it was shown that the three parameters of this extended KS approach can be determined by using CMDS. The resulting line-shape model, built from the corresponding collision kernels and completed by speed-dependent widths and shifts deduced from experiments, was successfully compared to measured Raman lines of $\mathrm{H}_{2}$ in $\mathrm{N}_{2}$ and $\mathrm{Ar}[136,138,139]$. The same approach was then applied to pure $\mathrm{H}_{2} \mathrm{O}$ and $\mathrm{H}_{2} \mathrm{O}$ in $\mathrm{N}_{2}$ [140-142]. For these systems, where the decay times of the auto-correlation functions of the velocity modulus and orientation are close to each other [140,141], a single $\alpha$ parameter was used. Its value and that of $v_{\mathrm{VC}}$ were deduced from CMDS. These calculations also enabled $[140,141]$, for the first time, quantification of a parameter that describes the correlation between collision-induced changes of the translational and rotational motions. The speeddependent widths and shifts, other components of the kinetic equation, were calculated [140,141] using the RB formalism (Sec. 3.3.4). The resulting line shapes are in excellent agreement with those measured for many transitions of pure $\mathrm{H}_{2} \mathrm{O}$ and $\mathrm{H}_{2} \mathrm{O}$ in $\mathrm{N}_{2}$, under various pressure conditions [140-143]. It was in particular shown, using an empirical correction procedure similar to that applied to the CMDS predictions of CO line-shapes ( $c f$ [125] and Sec. 3.2.1), that this approach leads to absolute predictions of the profiles of $\mathrm{H}_{2} \mathrm{O}$ lines broadened by $\mathrm{N}_{2}$ [143] wthin better than $0.5 \%$. For completeness, recall that the (monoparametric) KS model was used to describe the collision kernel of pure $\mathrm{H}_{2}$ in [144]. In this study, a purely numerical solution [145] of the kinetic equation was implemented for the first time and satisfactory agreement with measured spectra was obtained over a broad pressure range.

\subsection{2b The billiard-ball (BB) approximation for the velocity collision kernel}

The billiard-ball (BB) model was derived assuming a hard-sphere interaction potential $[146,147]$ and the associated velocity collision kernels are given in analytical form [148]. In an analysis of $\mathrm{O}_{2}$ spectra measured via FS-CRDS [149], it was shown that the use of the BB model for the description of velocity changes yielded the highest quality-of-fit (as defined in Ref. [11]) ever reached for a molecular line. The speed-dependent billiard-ball (SDBB, [150]) profile was used to quantify the ability of various line-shape models to reproduce a benchmark theoretical profile [151]. It also enabled to estimate possible systematic errors on Doppler width measurements [149] for the spectroscopic determination of the Boltzmann constant ( $c f$ also Sec. 5.2.1) and to determine the frequencies of unperturbed rovibrational transitions [152]. This profile was also used [153] to rescale the narrowing parameter of the HC model and improve the treatment of velocity-changing collisions in the Hartmann-Tran profile (Sec. 3.2.4a). Note that the large discrepancy between quantum computations of the line widths and those measured for $\mathrm{H}_{2}$ perturbed by Ar obtained in [154] was solved [155] by using the SDBB profile which properly handled velocity- and speed-changing collisions ( $c f$ Fig. 6). Using the SDBB model for lines of $\mathrm{H}_{2}$ perturbed by $\mathrm{He}$, it was shown [156] that comparisons of measured and calculated full line shapes enable to discriminate various PESs while considering only pressure-broadening and -shifting coefficients is not sufficient. 

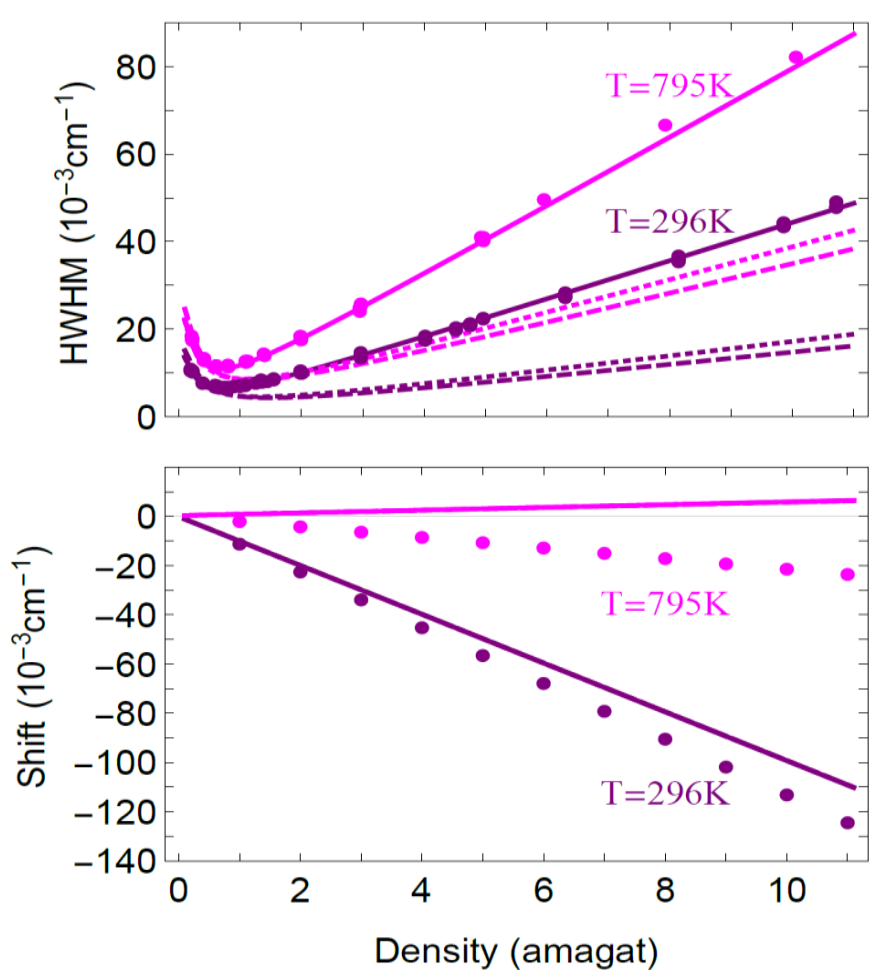

Fig. 6: Apparent width (HWHM) and shift of the isotropic Raman $Q(1)$ transition of the fundamental band of $\mathrm{H}_{2}$ perturbed by Ar for various temperature and density conditions. The solid lines represent the results of calculations using the SDBBP, while the points correspond to the experimental values [157,158]. The dashed and dotted lines were obtained from calculations in which the velocity changing-collision model was the phenomenological soft and hard-collision models, respectively. After [155].

\subsection{2c Velocity collision kernel calculated from CMDS}

CMDS were used to obtain the velocity collision kernel for pure $\mathrm{H}_{2}$ in [144]. As mentioned in Sec. 3.2.1, these calculations provide the time evolution of the center-of-mass velocity of each molecule. It is thus possible to follow the number of molecules, among those having a given velocity at time $t$, which have changed to another velocity at time $t^{\prime}$. This gives detailed information on the velocity changes from which the associated kernels can be deduced [144]. The latter were compared with those calculated using the KS model (Sec. 3.3.2a) showing that this last/approach does not well describe velocity-to-velocity changes (Fig. 7) although it leads to (practically) the same averaged correlation function of the molecular velocity [144]. Comparisons between measured line shapes of Raman and quadrupole lines of pure $\mathrm{H}_{2}$ and those calculated using the KS and CMDS collision kernels showed that the latter lead to better and very satisfactory agreement with measurements [144].

Figure 7 shows a comparison between velocity collision kernels computed for $\mathrm{H}_{2}-\mathrm{H}_{2}$ interactions using CMDS, the BB and KS models. As demonstrated in [159] and shown by this plot, for $\mathrm{H}_{2}-\mathrm{H}_{2}$ (and also $\mathrm{H}_{2}-\mathrm{Ar}$ ), the kernels calculated with the $\mathrm{BB}$ model are in excellent agreement with those predicted by CMDS, while the KS function leads to values which are independent of the relative orientation change between the velocities before and after the collision. 


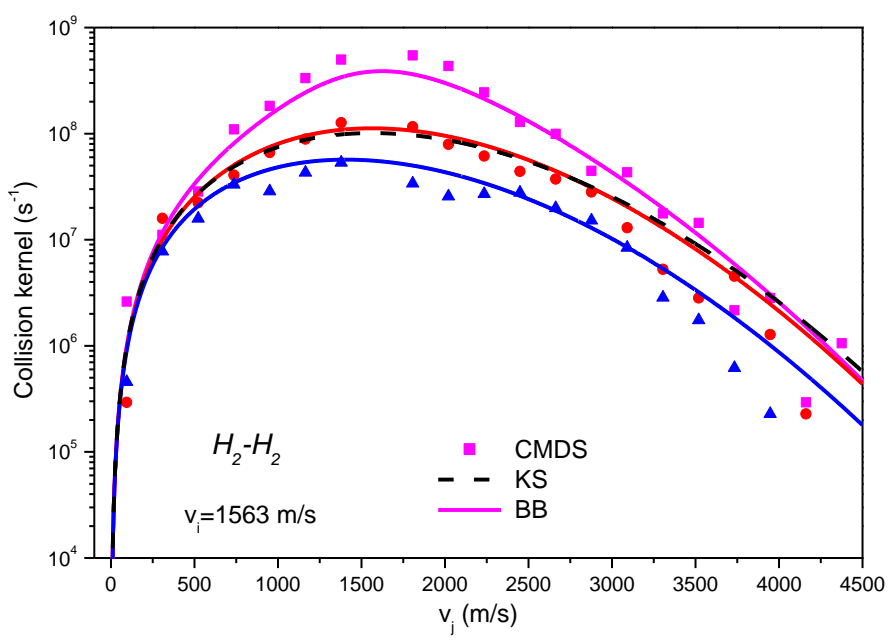

Fig. 7: Velocity collision kernels for $\mathrm{H}_{2}-\mathrm{H}_{2}$ calculated by CMDS (symbols), by the BB approach (solid lines) and using the $K S$ model (dash line) at $296 \mathrm{~K}$ and 1 amagat. The results, for the initial speed $v_{i}=1563$ $\mathrm{m} / \mathrm{s}$, are plotted $v s$ the final speeds $v_{j}$. Magenta, red and blue results correspond to angles between the initial and final velocities of 36, 90 and $144^{\circ}$, respectively. The same value (dashed black line) for the three angles is obtained with the KS model). After [159].

\subsubsection{Relativistic and dispersion correction to line-shape models}

Models have been proposed recently to account for some refined effects that affect the line shapes. The proposed corrections, discussed below, will likely be crucial for ultra-precise Doppler thermometry ( $c f$ Sec. 5.2.1) and molecular spectroscopy for fundamental studies. Although these effects have not yet been experimentally verified, it may become possible in the future to observe them through measurements capable of detecting relative changes of the line shape at the typical $10^{-6}$ level (see below).

The relativistic formula for the Gaussian profile was given in [160] and an extension to the VP was derived in [161]. It was demonstrated that relativistic effects lead to a line asymmetry and can modify the profile at the $10^{-6}$ level [161] at room temperature (with modifications that increase with temperature). Equivalent formulas for more advanced (nonVoigt) relativistic profiles have not been proposed so far.

The dependence of the Doppler shift on frequency, caused by dispersion [162,163] and by light frequency variation over the spectral line shape, is another refined effect that needs experimental demonstration. A model taking this into account was derived for a Gaussian line shape [163], showing that it leads to a line asymmetry, affects the line shape at the level of $10^{-}$ 5 and may influence line-position measurements at the $\mathrm{kHz}$ level. Again, for future applications, dispersion corrections are needed for profiles also influenced by collisional effects.

\subsubsection{Phenomenological line-shape models}

Models that explicitly express the line shape analytically as a parameterized function with a few (adjustable) parameters are very useful. They can be fitted to measured spectra (Sec. 4.1), providing parameters which can be archived in databases (Sec. 4.5), opening the route for use in practical applications (Sec. 5). In this section, we review some recent advances and results obtained with various phenomenological profiles used to represent the shape of isolated lines.

The simplest line shapes are the Doppler and Lorentz profiles. They depend on the Doppler width and on the "usual" pressure-broadening and -shifting coefficients, respectively. The convolution of these two profiles results in the Voigt (V) profile, which captures the inhomogeneous collision-induced line broadening that encompasses all velocity classes. It is then possible to account for the collision-induced changes of the radiating-molecule velocity by assuming Soft (SC) or Hard (HC) Collisions. These assumptions lead to the Galatry [135] and Rautian [126,134] line-shapes, respectively. Concerning the Speed Dependences (SD) of 
the collisional width and shift, they are usually modeled using a quadratic (q) $[164,165]$ or a hypergeometric (h) $[166,167]$ law. Then, models for velocity-changes and speed-dependences can be combined, leading to a large variety of models such as (using the acronyms introduced above) the qSDVP, the qSDHC and the hSDSC profiles, for instance. Note that it is also possible to introduce a partial Correlation (pC) [126] between the collision-induced changes of the radiator velocity and those of its rotational (speed and phase) state. Figure 8 (see also that in Sec. 5.2.2) shows examples of fits of measured spectra with a variety of phenomenological lines-shape models (in which LM has been included). As can be seen, the VP leads to large, but not atypical, residuals. Furthermore, Fig. 8 shows that not only the speed-dependence but also the velocity changes and the correlation must be taken into account in order to obtain the best fit of the high quality observed line-profiles.

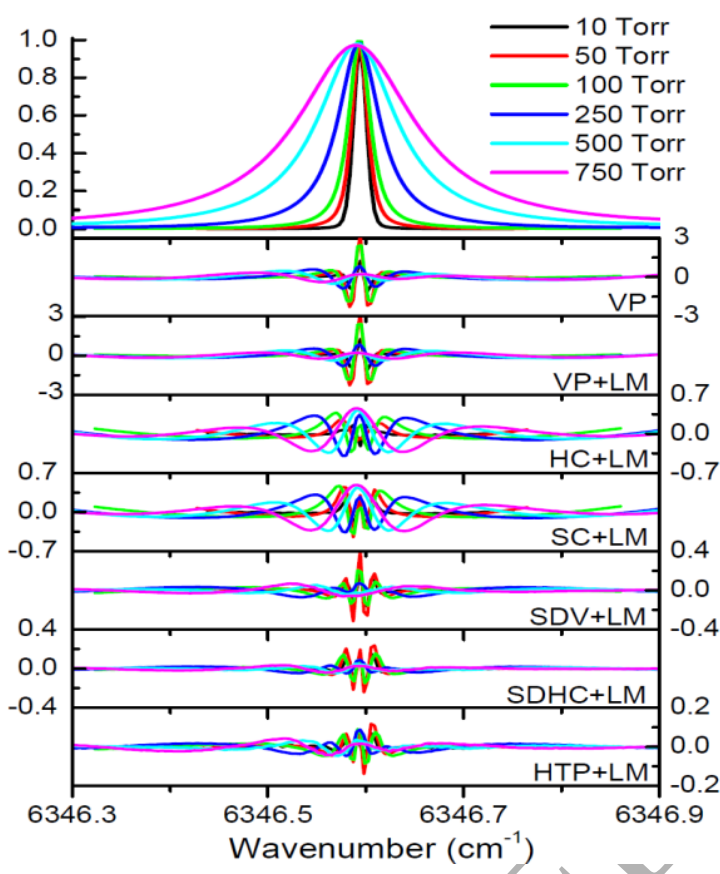

Fig. 8: Top panel. Peak-normalized absorption spectra of the 3-O P(1) lines of ${ }^{12} \mathrm{C}^{16} \mathrm{O}$ broadened by Kr measured at room temperature and various total pressures. Bottom panels: Residuals (in \% of the peak absorption) obtained from multispectrum fits [99] of the measured spectra with various lineshape models. Note that different vertical scales are used for the various residuals. After [168].

Many models can be found in Table III.6 and Sec. III.3 of [1] and in Table 1 of [169] with detailed descriptions in the references therein. All of these approaches have existed for awhile, but the most sophisticated ones were seldom used until recently only because they involve numerous parameters. Indeed, since measurements carry noise, the solutions to make the retrieval of all the model parameters as unambiguous as possible are [169]: to use spectra of the best quality achievable, to record them over a pressure range as broad as possible (ideally from the practically purely Doppler to the almost purely collisional behavior of the line shape), and to treat them using a multispectrum fitting procedure $[99,170]$.

\subsection{4a The Hartmann-Tran profile}

Starting from the partially-correlated speed-dependent hard-collision model [171], Hartmann, Tran and co-workers combined it [169] with quadratic speed dependences $[164,165]$ for the line width and shift. The resulting pCqSDHC profile accounts for the Doppler effect, the influence of velocity changes caused by collisions, the speed dependences of the line width and shift and the correlation between velocity- and internal-state changes. Using $a b$ initio calculated line shapes of various molecular systems as references, [169] showed that the pCqSDHC can reproduce most of them over a wide pressure range with a precision better than $\approx 0.1 \%$. Furthermore, [169] demonstrated that this line profile can be written as a combination of complex error functions for which many computer-efficient routines are available (eg [172-177]). Therefore, the pCqSDHCP can be easily and quickly computed [178], contrary to most of the other sophisticated line shapes, which enables the use 
of this profile for computationally intensive calculations required for radiative transfer and remote sensing applications. In addition, by setting some parameters to zero, the pCqSDHC profile reduces to several simpler line shapes [169], including the widely used qSDVP and the HC model. Because of its numerous useful attributes, the pCqSDHCP profile was recommended by an IUPAC task group as the reference line shape to be used in highresolution spectroscopy [179]. Now known as the "Hartmann-Tran" profile (HTP), it is increasingly used (Sec. 4.1) and the HITRAN database now enables the storage of its parameters (Sec. 4.5.1).

\subsection{4b Temperature dependence of the line-shape parameters}

Relatively little has been done on the temperature dependences of the line-shape parameters other than the usual broadening and shifting coefficients associated with the VP (as confirmed by the table in Sec. 4.1).

Concerning the speed-dependence, a relation between the temperature dependence of the collisional broadening and that of the parameter describing its quadratic speed dependence was given in [180]. It was obtained assuming that the dependence on the relative speed of the collisional broadening is also quadratic. In this particular case, it was shown [181] that the ratio of the broadening speed-dependence parameter to the pressure-broadening coefficient should be independent of $T$. This result was experimentally confirmed to within a few percent for variable-temperature measurements of $\mathrm{CO}_{2}$ line shapes in the $1.6 \mu \mathrm{m}$ region [181]. However, some other experimental studies [103,182-184] have shown that the temperature dependences of the line broadening and of its quadratic speed-dependent component are significantly different. For the Dicke-narrowing parameter (generally denoted by $v_{\mathrm{VC}}$ ), a power law for its temperature dependence was used in a few studies (eg [103,182,185,186]) that have investigated this issue. The corresponding experimental results show that the temperature dependence exponent of $v_{\mathrm{VC}}$ can be significantly different from that of the line broadening (eg 1.2 versus 0.75 on average in [103]). Finally, to the best of our knowledge, there is no experimental or theoretical result available for the temperature dependences of the speed dependence of the pressure shift and of the correlation parameter.

This sub-section shows that the influence of temperature on the collisional parameters of refined isolated line profiles (beyond the VP) remains poorly known. New (very careful) experimental investigations are needed to clarify this important issue to which the theoretical approach of Secs. 3.2.1, 3.2.2 and 3.3 may also bring interesting information.

\subsection{4c Quadratic versus hypergeometric speed dependences}

The quadratic speed-dependence (qSD) $[164,165]$ is different from the hypergeometric (hSD) model [166,167]. The latter is more physically based since it results from an assumption directly concerning the intermolecular potential. Using high quality spectra of $\mathrm{O}_{2}$ lines at low pressure, it was shown [187] that the pChSDHC model leads to slightly better fits of the observed line-shapes than the HTP (ie pCqSDHCP). Similarly, in situations involving large speed-dependence effects, such as a high rotational quantum number line and/or a light active molecule interacting with a heavy perturber, the hypergeometric model also better reproduces the measured spectra than does the quadratic model [188]. A detailed comparison between the $\mathrm{pChSDHC}$ and the pChSDHC profiles was also made in [189] using high quality spectra of $\mathrm{H}_{2} \mathrm{O}$ lines in the Dicke-narrowed regime. The same fit qualities were obtained with the two models. For the retrieved parameters, while the integrated absorbances and speedaveraged pressure-broadening and -shifting coefficients are very consistent, the other parameters are quite different [189]. With respect to the hypergeometric model, the line narrowing due to the speed dependence is underestimated by the quadratic approximation whereas the velocity-changing frequency is overestimated [189]. In other measurements of self-broadened $\mathrm{H}_{2} \mathrm{O}$ lines, it was shown that $\mathrm{HC}$ profiles with the quadratic and 
hypergeometric speed dependences gave nearly identical results, reproducing the spectra with residuals at the $0.01 \%$ level [8].

\subsection{4d Kochanov's approach}

In [190] and references therein, a new line-profile model was proposed. It is based on quantum mechanical collision-integral kernels calculated for an intermolecular interaction potential proportional to $1 / r^{\mathrm{n}}$, with $r$ the intermolecular distance (thus much simpler that those that can be implemented in the codes, such as MOLSCAT [191], discussed in Sec. 3.3.1). Specifically, the collisional kernels were modeled and then fitted to the quantum-mechanical calculated kernels. This model accounts for the Dicke narrowing, the effects of small- and large-angle scattering collisions [192], and the speed dependences of the collisional relaxation rates. The resulting profile involves a plethora of parameters likely to be fitted to experiments but, to the best of our knowledge, its ability to accurately describe measurements has not been proven so far.

\subsection{Pressure-broadening and -shifting coefficients}

The pressure-broadening $(\gamma)$ and -shifting $(\delta)$ coefficients are the first quantities to be known for calculations of spectra when the Doppler effect is not dominant. Thus, for decades, efforts have been made to determine these parameters and subsequently populate databases. Until relatively recently, the VP was mostly used and knowledge of the values $\langle\gamma\rangle$ and $\langle\delta\rangle$ averaged over the velocity distribution was sufficient. With the increase of precision requirements and the now well-recognized fact that speed-dependence effects are important, these two parameters have become insufficient to capture the pressure dependence of line shapes. One then needs to know the dependences of $\gamma$ and $\delta$ on the absorbing-molecule speed, which are ingredients of a variety of refined line-shape models (Sec. 3.2 above and III.4 of [1]). We focus here on their calculation for which we review recent theories and results, while available experimental determinations are presented in Sec. 4.1.

When an intermolecular potential is available, many formalisms exist for calculations of $\gamma\left(v_{\mathrm{r}}\right)$ and $\delta\left(v_{\mathrm{r}}\right)$, where $v_{\mathrm{r}}$ denotes the relative speed. They are adapted to systems of various complexity, in terms of the symmetry of the molecules, of the number and nature of the significantly populated levels and of the parameterization of the intermolecular potential. For instance, while fully quantum computations are tractable for He-broadened rotational lines of $\mathrm{CO}$ at $10 \mathrm{~K}$, they cannot be made for transitions of high overtones of pure $\mathrm{H}_{2} \mathrm{O}$ at $2000 \mathrm{~K}$, a case that can be treated with (much simpler and approximate) semi-classical models. The resulting dependences on the absorbing-molecule speed are then obtained by averaging $\gamma\left(v_{\mathrm{r}}\right)$ and $\delta\left(v_{\mathrm{r}}\right)$ over the perturber velocity [167]. Until recently, limitations in computing power have restricted the use of fully quantum approaches to very simple collisional pairs, a constraint progressively relaxed as shown in the next section. For more complex systems, progress has been made thanks to improvements of the RB theory brought by the inclusion of line-coupling, as discussed in Sec. 3.3.2. At the same time, Gordon's fully classical method was resurrected by S. Ivanov and enabled calculations of pressure-broadening coefficients for linear molecules (Sec. 3.3.3). Finally, for (very complex) molecular pairs for which other methods are inapplicable or too costly, the "usual" RB approach remains useful as shown in Sec. 3.3.4. Note that, while the refined RB approach has been tested only at room temperature, it is not the case of the other theories discussed below. For instance, the broadening of $\mathrm{C}_{2} \mathrm{H}_{2}$ and $\mathrm{N}_{2}$ lines by $\mathrm{H}_{2}$ was studied from $77 \mathrm{~K}$ to $2000 \mathrm{~K}[193,194]$ while broadening of pure $\mathrm{N}_{2}$ and of $\mathrm{CO}_{2}$ in $\mathrm{Ar}$ was investigated [195,196] in the 77-2400 $\mathrm{K}$ and $77-765 \mathrm{~K}$ ranges, respectively. In these references, quantum, fully classical and the usual RB semi-classical approaches were used for comparisons with measured values. Agreement within experimental uncertainties was obtained in most cases except with the RB formalism. However, if some 
intermolecular-potential parameters are tuned, the latter provides a satisfactory description of the temperature dependence. This may explain why this computationally cheap method remains so popular from a pragmatic point of view ( $c f$ Sec. 3.3.4).

\subsubsection{Quantal approaches}

The approaches, such as the close-coupling (CC) method, in which all degrees of freedom (translation, rotation, etc) are treated quantum mechanically provide the most reliable predictions of $\gamma\left(v_{\mathrm{r}}\right)$ and $\delta\left(v_{\mathrm{r}}\right)$ if an accurate intermolecular potential is used. A description of the CC method and of various decoupling approximations, such as the coupled states (CS), the decoupled $l$-dominant and the effective potential methods, as well as the infinite-order sudden (IOS) approximation, can be found in [197] and Sec. IV.3.5 of [1]. Several computer codes have been developed (eg MOLSCAT and PMP MOLSCAT [191], MOLCOL [198], HIBRIDON [199]) to calculate the matrix elements of scattering operators at various levels of accuracy. MOLSCAT and its parallelized version PMP MOLSCAT treat collisions between closed-shell species ranging from rigid diatom-atom to rigid diatom-asymmetric top. HIBRIDON and MOLCOL solve the close-coupled equations for both closed- and open-shell systems. From the scattering-matrix elements provided by such codes, built-in functions or post-processor programs generate relative kinetic-energy-dependent pressure-broadening and -shifting generalized cross sections for the considered type of spectroscopy. They can then be transformed [167] into values that depend on the speed of the radiating molecule. In addition, the averaged broadening and shifting coefficients at various temperatures can be obtained assuming a Maxwell-Boltzmann distribution.

Because of its computational cost, the CC formalism was mainly applied to simple systems [diatom-atom or (light) diatom-(light) diatom] while more approximate methods, like the CS or IOS, were used for more complicated cases (symmetric top-atom or asymmetric top-atom). The increase in computer speed, the use of clusters, of parallelization, of job arrays, etc, in conjunction with the availability of more accurate full-dimensional potential-energy surfaces (PES) now permit more rigorous treatments of all kinds of systems. For instance, these advanced computational capabilities have enabled, for $\mathrm{H}_{2}-\mathrm{He}$, consideration of the effects of the centrifugal distortion of the PES or of rovibrational potential coupling terms $[156,200]$. PESs for the active molecule in the ground state or in an excited electronic state also have enabled calculations of pressure-broadening and -shifting coefficients for rotational, rovibrational and rovibronic lines [201,202]. Furthermore, CC predictions for asymmetric-top molecules in a bath of diatomics are nowadays feasible. Good examples are calculations for $\mathrm{H}_{2} \mathrm{CO}$ and $\mathrm{H}_{2} \mathrm{O}$ in $\mathrm{H}_{2}$ [203-206], systems that were never investigated at this level of accuracy before. Let us mention in particular the $\mathrm{CC}$ pressure-broadening and -shifting (which are more difficult to converge) coefficients of pure rotational lines of $\mathrm{H}_{2} \mathrm{O}$ in $\mathrm{H}_{2}$ calculated over a wide temperature range [204]. The next challenge would be to repeat such calculations for rovibrational lines while taking the centrifugal distortion of the potential into account. Finally note a recent work [207] in which the broadening and shifting coefficients as well as the real and imaginary parts of the Dicke narrowing parameter have been calculated for $\mathrm{H}_{2}$ in $\mathrm{He}$. This opens the route to refined simulations of isolated lines shapes following [208,209]. Table 1 regroups references in which various types of quantum calculations are presented. In some of these studies, both measurements and calculations were made, while the others compare theoretical results with previously published experimental data. The temperature dependences are also investigated in a few of them [156,193,194,210-212]. Generally speaking, when the calculations are performed using an accurate ab initio PES, the CC values for the widths and the shifts are accurate, to within about $1 \%$, at all temperatures except for very low ones. Indeed, there is an unexplained significant overestimation of the broadening by predictions in 
the very low temperature domain (below $10 \mathrm{~K}$ ), as shown in [213] and further discussed in Sec. IV.3.5b of [1]. Finally, an example of the quality of predictions in the case of $\mathrm{N}_{2^{-}}$ broadened $\mathrm{C}_{2} \mathrm{H}_{2}$ lines is given in Sec.3.3.3.

\begin{tabular}{|c|c|c|c|}
\hline System & Refs. & Method & Details \\
\hline $\mathrm{C}_{2} \mathrm{H}_{2}-\mathrm{Ne}$ & {$\left[210^{\#}\right]$} & $\mathrm{CC} / \mathrm{CS}(*)$ & PBC for infrared R lines. 77 and $1000 \mathrm{~K}$. \\
\hline $\mathrm{C}_{2} \mathrm{H}_{2}-\mathrm{H}_{2}$ & {$\left[214^{\#}\right]$} & $\mathrm{CC} / \mathrm{CS}(*)$ & PBC for infrared R lines. $77-2000 \mathrm{~K}$. \\
\hline $\mathrm{C}_{2} \mathrm{H}_{2}-\left(\mathrm{H}_{2}, \mathrm{D}_{2}\right)$ & {$\left[211^{\#}\right]$} & $\mathrm{CC}(*)$ & PBC for the $\mathrm{R}(\mathrm{j}=0-20)$ IR lines. 173,195 and $295 \mathrm{~K}$ \\
\hline $\mathrm{C}_{2} \mathrm{H}_{2}-\mathrm{H}_{2}$ & [215] & $\mathrm{CC}(*)$ & $\begin{array}{l}\text { PBC for isotropic Raman lines at } 143 \mathrm{~K} \text { and IR lines } \\
\text { at } 173 \text { and } 295 \mathrm{~K}\end{array}$ \\
\hline $\mathrm{C}_{2} \mathrm{H}_{2}-\mathrm{H}_{2}$ & [193] & $\mathrm{FC} / \mathrm{SC} / \mathrm{CC} / \mathrm{CS}(*)$ & PBC for isotropic Raman lines. $77-2000 \mathrm{~K}$. \\
\hline $\mathrm{C}_{2} \mathrm{H}_{2}-\mathrm{N}_{2}$ & [216] & $\mathrm{FC} / \mathrm{SC} / \mathrm{CC} / \mathrm{CS}(*)$ & PBC for IR and Raman lines. 150 and $300 \mathrm{~K}$ \\
\hline $\mathrm{C}_{2} \mathrm{H}_{2}-(\mathrm{Ar}, \mathrm{Kr}, \mathrm{Xe})$ & {$\left[217^{\#}\right]$} & $\mathrm{CC}(*)$ & PBC for IR lines. 77-400K \\
\hline $\mathrm{CO}-\mathrm{H}_{2}$ & {$\left[205^{\#}\right]$} & $\mathrm{CC}(+\mathrm{RPA})$ & $30 \mathrm{CO}$ lines between 115 and $3500 \mathrm{GHz} 200-3000 \mathrm{~K}$ \\
\hline${ }^{13} \mathrm{CO}_{2}-\mathrm{He}$ & {$\left[212^{\#}\right]$} & $\mathrm{CC} / \mathrm{CS}(*)$ & PBC for the $\mathrm{R}(10)$ and $\mathrm{P}(2) \mathrm{IR}$ lines. $85-300 \mathrm{~K}$. \\
\hline $\mathrm{DCO}^{+}-\mathrm{He}$ & [218] & $\mathrm{CC}$ & $\begin{array}{l}\text { PBXS \& PSXS for the } \mathrm{j}=2 \leftarrow 1 \text { to } 7 \leftarrow 6 \text { hyperfine } \\
\text { rotational transitions. }\end{array}$ \\
\hline $\mathrm{H}_{2} \mathrm{O}-\mathrm{H}_{2}$ & [203] & $\mathrm{CC}$ & $\begin{array}{l}\text { PBC for the } 1_{11} \leftarrow 0_{00} \text { and } 1_{10} \leftarrow 1_{01} \text { rotational lines. 65- } \\
220 \mathrm{~K}\end{array}$ \\
\hline $\mathrm{H}_{2} \mathrm{O}-\mathrm{H}_{2}$ & {$\left[204^{\#}\right]$} & $\mathrm{CC}$ & $\begin{array}{l}\text { PBC \& PSC for the } 1_{11 \leftarrow} \leftarrow 0_{00}, 1_{10} \leftarrow 1_{01} \text { and } 1_{11} \leftarrow 2_{02} \\
\text { rotational lines. } 20-250 \mathrm{~K}\end{array}$ \\
\hline $\mathrm{H}_{2} \mathrm{O}-\mathrm{H}_{2}$ & {$\left[205^{\#}\right]$} & $\mathrm{CC}(+\mathrm{RPA})$ & $\begin{array}{l}\text { PBC for } 228 \mathrm{H}_{2} \mathrm{O} \text { transitions between } 10 \text { and } 20000 \\
\mathrm{GHz} .200-3000 \mathrm{~K}\end{array}$ \\
\hline $\mathrm{H}_{2} \mathrm{O}-\mathrm{He}$ & [219] & PA) & $\begin{array}{l}\text { PBC for } 21 \text { transitions between the eight lowest } \\
\text { rotational levels of ortho and para } \mathrm{H}_{2} \mathrm{O} .20-120 \mathrm{~K}\end{array}$ \\
\hline $\mathrm{HCN}-\mathrm{He}$ & {$\left[213^{\#}\right]$} & $\mathrm{CC}$ & $\begin{array}{l}\text { PBC \& PSC for hyperfine components of the } \mathrm{j}=1 \leftarrow 0 \\
\text { and } 2 \longleftarrow 1 \text { rotational lines. } 1.3-20 \mathrm{~K}\end{array}$ \\
\hline $\mathrm{H}_{2} \mathrm{CO}-\mathrm{H}_{2}$ & [206] & $\mathrm{CC} / \mathrm{CS}$ & PBC for the $3_{13} \leftarrow 2_{12}$ rotational transition. $10-25 \mathrm{~K}$ \\
\hline $\mathrm{HCO}^{+}-\mathrm{He}$ & {$\left[220^{\#}\right]$} & $\mathrm{CC}$ & $\begin{array}{l}\text { PBC \& PSC for the } \mathrm{j}=1 \leftarrow 0 \text { to } 6 \leftarrow 5 \text { rotational lines. } \\
88 \mathrm{~K}\end{array}$ \\
\hline $\mathrm{H}_{2}-\mathrm{Ar}$ & $\begin{array}{l}{[154]} \\
{[155]}\end{array}$ & $\mathrm{CC}$ & $\begin{array}{l}\text { Speed dependence of the PBS \& PSC of the isotropic } \\
\text { Raman Q(1) line }\end{array}$ \\
\hline$\left(\mathrm{H}_{2}, \mathrm{D}_{2}\right)-\mathrm{He}$ & [156] & & $\begin{array}{l}\text { PBC and PSC for the } \mathrm{S}_{0}(0) \text { line of } \mathrm{H}_{2} \text { and } \mathrm{v}=1 \leftarrow 0 \\
\mathrm{Q}(1) \text { line of } \mathrm{H}_{2} \text { and } \mathrm{D}_{2} \cdot 10-3000 \mathrm{~K} \text {. }\end{array}$ \\
\hline $\mathrm{H}_{2}-\mathrm{He}$ & [207] & & $\begin{array}{l}\text { PBC \& PSC and narrowing parameters for } \mathrm{v}=0 \rightarrow(1 \text { to } \\
5) \text { and } \mathrm{Q}(\mathrm{j}=0 \text { to 5) lines. } 10-2000 \mathrm{~K}\end{array}$ \\
\hline $\mathrm{N}_{2}-\mathrm{H}_{2}$ & {$\left[221^{\#}\right]$} & $\mathrm{CC} / \mathrm{CS}(*)$ & PBC for isotropic Raman Q lines. 77-580 K. \\
\hline $\mathrm{N}_{2}-\mathrm{H}_{2}$ & [194] & $\mathrm{FC} / \mathrm{SC} / \mathrm{CC} / \mathrm{CS}(*)$ & PBC for isotropic Raman lines. 77-2000 K. \\
\hline $\mathrm{N}_{2}-\mathrm{N}_{2}$ & {$\left[222^{\#}\right]$} & $\mathrm{CC} / \mathrm{CS}(*)$ & PBC for isotropic Raman lines. 77-298 K. \\
\hline $\mathrm{N}_{2}-\mathrm{N}_{2}$ & [195] & $\mathrm{FC} / \mathrm{SC} / \mathrm{CC} / \mathrm{CS}(*)$ & PBC for isotropic Raman lines. 77-2400 K. \\
\hline $\mathrm{N}_{2} \mathrm{H}^{+}-\mathrm{H}$ & {$\left[223^{\#}\right]$} & $\mathrm{CC}$ & $\begin{array}{l}\text { PBC \& PSC for the } \mathrm{j}=3 \leftarrow 2 \text { to } 6 \leftarrow 5 \text { hyperfine } \\
\text { rotational transitions. } 88 \mathrm{~K}\end{array}$ \\
\hline $\mathrm{OH}-\mathrm{Ar}$ & [202] & $\mathrm{CC}$ & $\begin{array}{l}\text { PBC \& PSC for rotational, vibrational and electronic } \\
\text { transitions. PESs for } X^{2} \Pi \text { and } A^{2} \Sigma^{+} \text {states } 296 \mathrm{~K}\end{array}$ \\
\hline $\mathrm{O}_{2}-\mathrm{H}$ & {$\left[201^{\#}\right]$} & CC (Hund case b). & $\begin{array}{l}\text { PBC \& PSC for transitions of the oxygen A-band. } \\
\text { Different PESs in the ground and excited states. } 296 \\
\text { K }\end{array}$ \\
\hline
\end{tabular}

Table 1: Bibliography in which quantal calculations of pressure-broadening (PB) and/or shifting (PS) coefficients $(C)$ or cross-sections $(X S)$ are presented. In column three FC stands for fully classical (Sect. 3.3.3.), SC for the semi-classical RB formalisms (Sect. 3.3.2 and 3.3.4), CC for close-coupling and CS for coupled states. (*) in column 3 denotes calculations with no vibrational dependence of the potential energy surface and thus no PSC. The references marked with a hash (eg [210\#]) are those in which both measurements and calculations have been made. 


\subsubsection{Refined semi-classical Robert-Bonamy (RB) formalism}

The RB formalism (Sec. 3.3.4) is the most widely used method for calculating collisional widths and shifts of isolated lines for complex molecules. However, although the non-diagonality of the Liouville scattering operator within the line space was known for years, the "usual" RB formalism ignores this property. As a first consequence, it significantly overestimates the line widths when compared with quantum calculations using the same intermolecular potential [193-195]. In addition, it does not enable prediction of the line couplings that result from the abovementioned non-diagonality. One of the most important contributions toward a refined formalism was presented in [224]. The proposed theory was applied to pure $\mathrm{NH}_{3}$ [225] and to $\mathrm{NH}_{3}$ diluted in $\mathrm{Ar}$ and $\mathrm{He}$ [226]. In both cases, the refinement of the $\mathrm{RB}$ formalism leads to a large decrease of the calculated widths. At the same time, a slightly different approach was proposed [227], based on the coordinate representation. It was first applied to isotropic Raman transitions of pure $\mathrm{N}_{2}$ [227,228] for which a comparison with benchmark quantum results [195,229] was possible. This analysis showed that accounting for the line coupling reduces the line widths by about $15 \%$ with a much better agreement with the quantum results. However, discrepancies remain, because of the semi-classical assumption which ignores exchanges between translational and internal degrees of freedom. Similar results were obtained for other linear molecules [230]. Finally, the case of symmetric tops with inversion symmetry, previously studied in $[225,226]$ with a different model, was investigated recently using the coordinate representation approach [231,232], with applications to parallel and perpendicular bands of pure $\mathrm{NH}_{3}$. Again, the improvement of the RB formalism leads to significant decreases (up to $30 \%$ in some cases) of the calculated widths and to much better agreement with experimental results [231,232], as shown in Fig. 9 (and 10 for a linear molecule).

Fig. 9: Relative differences between measured pressurebroadening coefficients of the ${ }^{s} Q(j, k=j)$ lines of the $v_{l}$ band of pure $\mathrm{NH}_{3}$ [233] and those calculated with (red) and without (black) taking into account the non-diagonality of the scattering operator(ie the line-coupling terms). After [231].
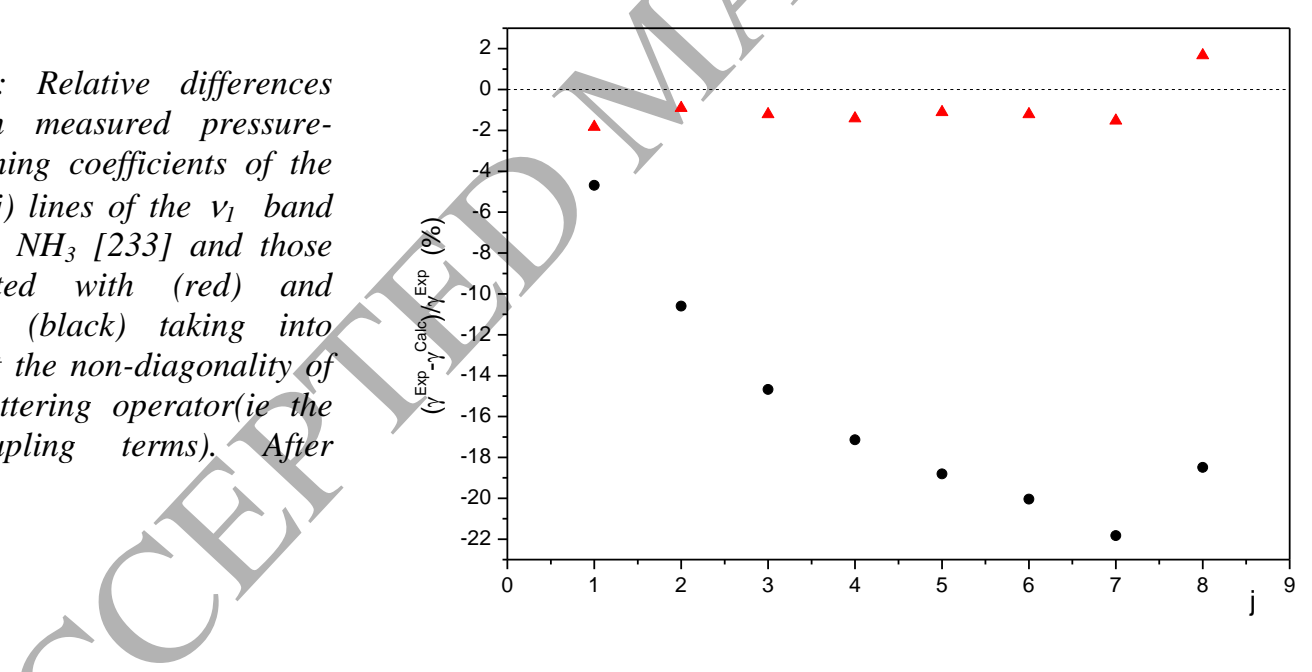

\subsubsection{Fully classical approach}

A series of papers [193-196,234-238] has resurrected the purely classical approach proposed by R.G. Gordon fifty years ago [239,240]. For several linear molecules, the resulting line-width predictions compare well with both experimental and quantum-calculated values, in much better agreement than the "usual" RB approach described in Sec. 3.3.4, as illustrated by Fig. 10. Another application of classical trajectories deserves to be mentioned: the demonstration of the influence of classically allowed quasi-bound complexes on the calculation of the widths and shifts for several molecular systems [241]. 


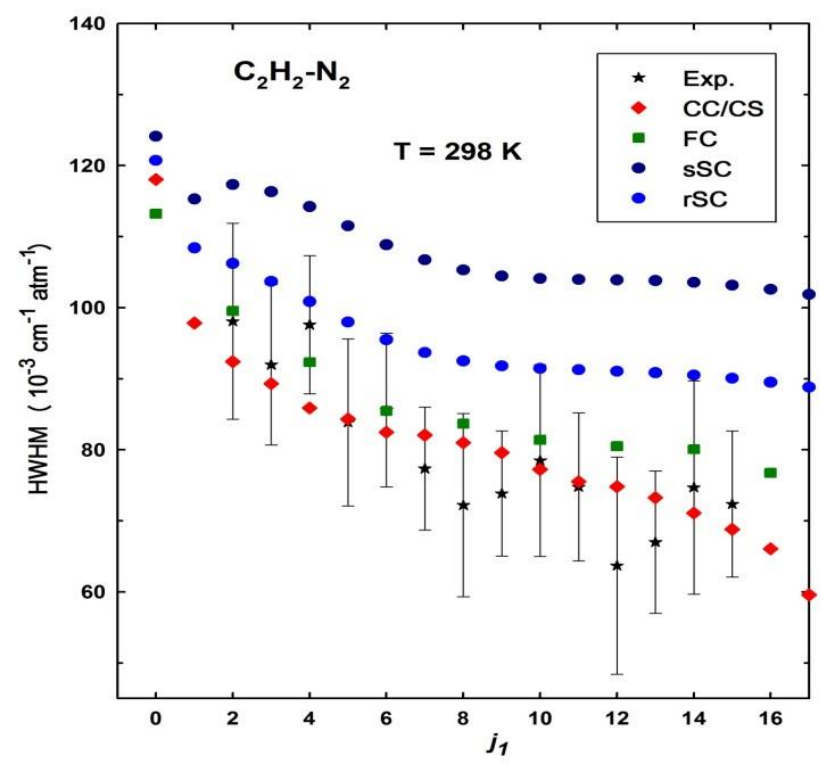

Fig. 10: Comparison of measured $\mathrm{N}_{2^{-}}$ broadening coefficients for isotropic Raman $Q\left(j_{1}\right)$ lines of $\mathrm{C}_{2} \mathrm{H}_{2}$ at $298 \mathrm{~K}$ with calculated values obtained using: full (Gordon's) classical method (FC), close coupling and coupled states method (CC/CS, Sec. 3.3.1), the refined RB semi-classical method (rSC, Sec. 3.3.2) and the "standard" RB semi-classical method as used in [242] (sSC, Sec. 3.3.4). After [216].

\subsubsection{Some recent applications of the "usual" Robert-Bonamy (RB) formalism}

The "usual" RB formalism, being based on the isolated-line approximation, only enables calculations of the widths and shifts. Despite its weaknesses (Sec. 3.3.2), this theory remains widely used because it can treat (very) complex molecular pairs, yielding reasonably good agreement with measurements at the price, in most cases, of some ad hoc adjustments of the intermolecular potential. Since its first version for linear molecules [243], extensions to other types of molecules and improvements (of the trajectory model and of the potential expansion) have been made, as reviewed in [244]. The bibliography on "RB pressure-broadened widths and shifts" is too vast to be exhaustively reported here, but Table 2 points to some recent or representative studies. For more information, the reader is invited to follow the bibliographical paths indicated in these studies, as well as those in [244] and Sec. IV.3.4e of [1].

\begin{tabular}{|c|c|c|c|c|}
\hline $\begin{array}{c}\text { Radiator } \rightarrow \\
\downarrow \text { Perturber }\end{array}$ & Linear & Symmetric-top & Asymmetric- top & $\begin{array}{c}\text { Spherical- } \\
\text { top }\end{array}$ \\
\hline Atom & $196,236]$ & --- & [261-267] & --- \\
\hline Linear & {$[193-195,245-252]$} & [257-259] & [268-282] & [288-291] \\
\hline Symmetric-top & --- & [260] & --- & --- \\
\hline Asymmetric-top & [253-256] & --- & {$[142,282-286]$} & --- \\
\hline Spherical-top & --- & --- & [287] & --- \\
\hline
\end{tabular}

Table 2: Some applications of the "usual" RB formalism to the calculation of pressurebroadening and-shifting coefficients.

\subsection{Line mixing}

We consider here, as in [292] and Chapt. IV of [1], the line-mixing (LM) process (also sometimes denoted as line coupling) in which exchanges of intensity between the lines result from the collision-induced transfers of populations between rotational levels. Except for recently proposed approaches in which the spectrum itself is directly computed (Sec. 3.4.1) or predicted based on some adjustable parameters (Secs. 3.4.7 and 3.4.8), all others rely on calculation of the so-called relaxation matrix $W$ (which is constructed in the line space and whose off-diagonal elements describe the LM process) from which the spectrum can be obtained (Sec. IV.2.1 of [1]). For this purpose, all available methods were proposed a while 
ago, except for the one presented in Sec. 3.4.3. It is important to recall that, except for that of Sec. 3.4.1, all the other approaches assume the "impact approximation" and are thus limited to calculation of LM effects not too far from line centers. In addition, and again with the exception of those presented in 3.4.1, all calculations disregard the effects of the speed dependence and of the collision-induced changes of the radiator velocity which have been investigated for isolated lines only (Sec. 3.2). The remainder of this section is organized as follows. We first present approaches that enable a direct prediction of the spectrum (Sec 3.4.1) or of the relaxation matrix $W$ (Secs. 3.4.2-3.4.4) from knowledge of the intermolecular potential. We then consider those with which $W$ elements can be computed using some parameters that are deduced from fits of measured data (Secs. 3.4.5 and 3.4.6). Finally, other semi-empirical and less commonly used models are presented in the last two sections.

The remainder of this section shows that significant advances have been made in the last decade for the development of new theories and models as well as for the further test of existing ones. However, except for the approaches discussed in Secs. 3.4.1 and 3.4.5, all others have been recently tested by comparisons with experiments at room temperature only. Quantifying the ability of the promising refined Robert-Bonamy and fully classical models to describe the effects of temperature is thus of interest for future studies.

\subsubsection{Requantized Classical Molecular Dynamics Simulations}

The requantized CMDS proposed in [117] (Sec. 3.2.1) for calculations of isolated-line shapes were extended in [123] to enable direct prediction of the absorption over an entire band. For this, the direction of molecular rotation is considered to discriminate between Pand R-branch lines, and corrections are introduced so that the line positions and intensities match some chosen (true) values. The resulting model, límited to linear absorbing molecules, was tested [123] by comparison with measured spectra of $\mathrm{CO}_{2}$ at elevated pressures, for some infrared bands from $296 \mathrm{~K}$ to $480 \mathrm{~K}$ and for an isotropic Raman Q branch from room temperature up to $700 \mathrm{~K}$. In most cases, the predictions agree with measurements within $5 \%$, regardless of temperature, as illustrated by Fig. 11.

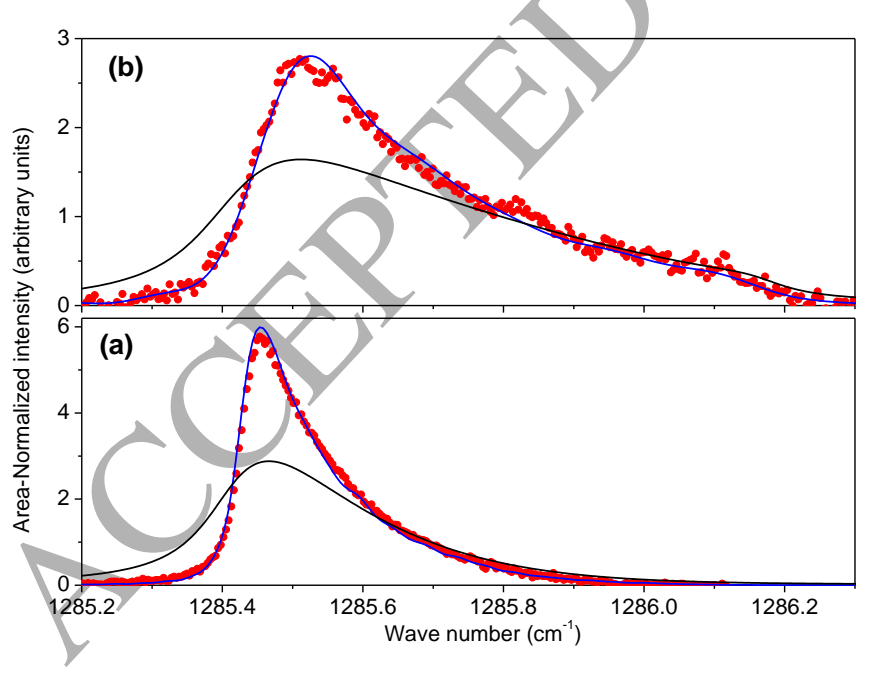

Fig. 11: Area-normalized intensities (in arbitrary units) in the $2 v_{2}$ isotropic Raman $Q$ branch of pure $\mathrm{CO}_{2}$ at: (a) $295 \mathrm{~K}$ and 0.5 amagat and $(b) 700 \mathrm{~K}$ and 1.0 amagat. Red symbols are measured values from [293], while the lines are calculated results obtained with the rCMDS model (blue) and neglecting LM (black). After [123].

\subsubsection{Quantal approaches}

As discussed in Sec. 3.3.1, computer codes have been developed for quantum calculations of matrix elements of the scattering operators at various levels of accuracy. Postprocessing programs then provide the diagonal and off-diagonal elements of the relaxation matrix $W$, depending on the type of spectroscopy (isotropic Raman diffusion, absorption, etc). While these tools have long existed, constructing a full $W$ matrix for molecular pairs using 
refined quantal approaches was intractable until recently. Thanks to the advances in computing power, this has progressively become feasible. A fully quantum calculation for the isotropic Raman spectrum of $\mathrm{N}_{2}$ at room temperature was made in [229] where it was shown that, for moderate values of the rotational quantum number, the convergence of CC/CS offdiagonal $W$ elements could be achieved, in good agreement with experimental data, therefore providing benchmark results for the test of more approximate formalisms (Secs. 3.4.3 and 3.4.4). Note that the case of isotropic Raman Q branches of linear molecules is "simple" when vibrational effects are neglected, because the real off-diagonal elements of $W$ are then simply the negative of the usual inelastic state-to-state collisional transfer rates. The oxygen A-band perturbed by helium was studied theoretically and experimentally in [201]. For the first time for an electronic transition, the relaxation matrix was obtained from a fully quantum mechanical approach, leading to good agreement with measurements. This is a significant and difficult step since it requires computations using (very complex) wave functions taking into account the rotational, vibrational and electronic degrees of freedom. The above-mentioned computations are thus close to the current limit of feasibility, which explains the rarity of applications of fully quantal approaches to LM processes.

\subsubsection{Refined semi-classical Robert-Bonamy formalism}

As discussed in Sec. 3.3.2, refinements of the "usual" $\mathrm{RB}$ formalism have been proposed, which do not neglect the non-diagonality of the Liouville scattering operator. This leads to better line-width predictions (Sec. 3.3.2), but also enables the calculation of the offdiagonal elements of the relaxation matrix $W$. Cherkasov investigated the case of molecules exhibiting hyperfine structure caused by the nuclear quadrupole interaction, by using his semi-classical theory, with applications to $\mathrm{CH}_{3} \mathrm{I}$ [294] and $\mathrm{CH}_{3} \mathrm{Cl}$ [295]. In most cases LM effects are weak. He later published a new version of his theory [224] which was successfully applied to the ${ }^{\mathrm{q}} \mathrm{Q}(\mathrm{j}, \mathrm{k})$ doublets in the $v_{1}$ band of pure $\mathrm{NH}_{3}[225]$ and to the calculation of $W$ elements for $\mathrm{NH}_{3}$ in $\mathrm{Ar}$ and $\mathrm{He}$ [226]. A slightly different approach, based on the use of the coordinate representation and choosing the orientations of pairs of molecules as a basis set in the Hilbert space (ie that of the states), was proposed in [227] in order to construct $W$ in the Liouville space (ie that of the lines). It was first applied to isotropic Raman spectra of pure $\mathrm{N}_{2}$ [228] with successful comparisons with benchmark quantum calculations of the $W$ matrix [229] (see figure in Sec. 3.4.4). Note that, as a consequence of the semi-classical assumption, a "sum rule" (eg [296] and Sec. 4.2.1 of [1]) that should be verified by the columns of the $W$ matrix is not satisfied. In order to correct this discrepancy, a "renormalization procedure" was proposed [296], in which the off-diagonal elements of $W$ are multiplied by factors determined from knowledge of the line-broadening coefficients. When this is done, the refined RB predictions compares well with quantum calculations, as mentioned above. However, without introducing this renormalization, the refined RB formalism can still provide semi-quantitative information on LM for complex systems. This approach was then extended to infrared spectra of linear molecules [230] with applications to parallel and perpendicular bands of $\mathrm{CO}_{2}$ in $\mathrm{N}_{2}$ [297]. By properly accounting for the influence of the vibrational angular momentum, the formalism leads to predictions of LM effects in good agreement with both measurements and ECS (Sec. 3.4.5) predictions for different types $(\Sigma \leftarrow \Sigma, \Pi \leftarrow \Sigma$, etc) of bands. The further extension to asymmetric tops has allowed the analysis of line couplings within $\mathrm{H}_{2} \mathrm{O}$ vibrational bands [298] and quantitative tools have been developed to determine the relative importance of the off-diagonal elements of $W$. This showed that most of the $\mathrm{H}_{2} \mathrm{O}$ lines in the pure rotational band are practically uncoupled while a few lines exhibit small but significant couplings. The case of symmetric tops with inversion symmetry, previously studied in $[225,226]$ with a different model, was investigated recently using the coordinate representation approach, with applications to parallel and perpendicular bands of pure $\mathrm{NH}_{3}$. 
The results show that this theory reproduces well the LM signatures (eg Fig. 12) and $W$ elements observed in some spectral regions of the $v_{1}$ and $v_{4}$ bands [299,300], as well as the influence of the vibrationally dependent inversion splitting [232].

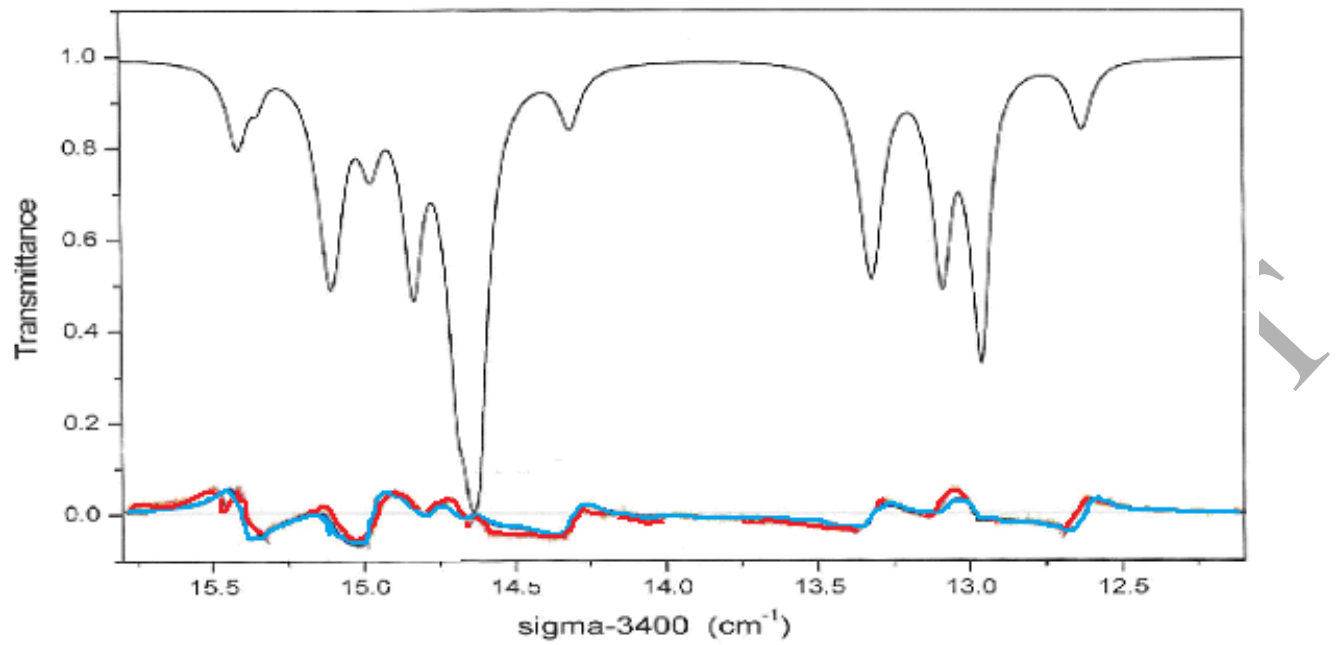

Fig. 12: LM signatures in the self-broadened $R(3, k)$ manifold of the $v_{1}$ band of pure $\mathrm{NH}_{3}$ at 50 Torr. The upper curve is the calculated transmittance. The bottom red (resp. blue) curve is five times the difference between the measured (resp. calculated) transmission and that fitted (resp. calculated) without the inclusion of LM. After [299].

\subsubsection{Fully classical formalism}

The classical approach proposed by R.G. Gordon fifty years ago [239,240,301] not only enables calculations of the pressure-broadening and -shifting coefficients (Sec. 3.3.3) but also of the off-diagonal elements of the relaxation matrix. This possibility has been used recently for predictions of LM effects in isotropic Raman spectra of pure $\mathrm{N}_{2}$ [237]. For this system, good agreement was obtained (Fig. 13) with results of a benchmark quantum calculation [229], thus opening the route to the treatment of more complex molecular pairs.

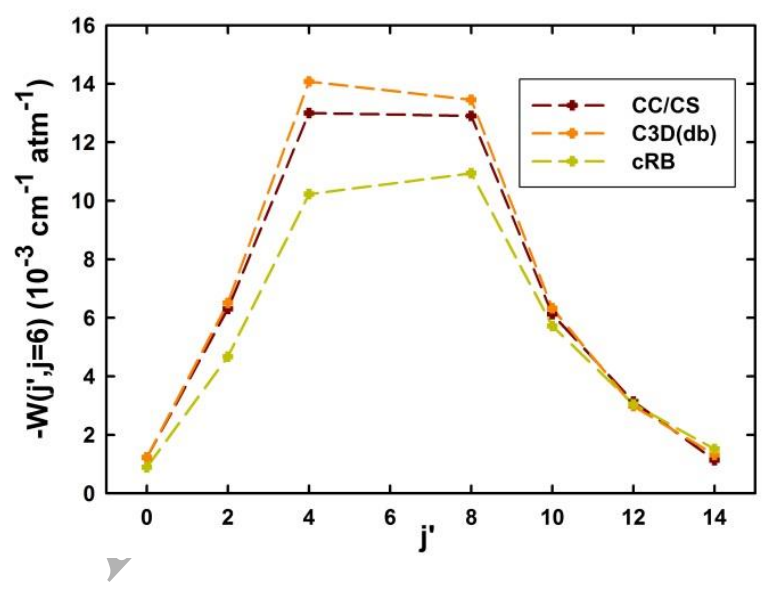

Fig. 13: Computed relaxation-matrix elements $W\left(j^{\prime} \leftarrow j=6\right)$ between the $Q(6)$ lines and various $Q\left(j^{\prime}\right)$ for $\mathrm{N}_{2}-\mathrm{N}_{2}$ at $298 \mathrm{~K}$. CC/CS denote the quantum results from [229], $c R B$ the corrected-RB predictions from [228] and $C 3 D(d b)$ the results of classical calculations. After [237].

\subsubsection{Dynamically based scaling laws}

The Infinite-Order Sudden (IOS) approximation and the more refined Energy-Corrected Sudden (ECS) scaling law have been used for the modeling of LM effects within various spectral features and for many molecular systems (Sec. IV.3.3 and Table IV.9 of [1]). These approaches construct the real-valued elements of the relaxation matrix $W$ from a limited set of parameters whose values can be calculated but are generally deduced from fits of measured line-broadening data. 
The quality of the ECS approach was further tested in studies devoted to a few linear molecules. For $\mathrm{CO}_{2}$, the models described in [296] and [302,303] were used for quite successful further comparisons with measurements in the central regions of many bands over wide ranges of temperature and pressure [304-315]. A successful application was also made to some bands of OCS in He at elevated pressures [316]. For $\mathrm{O}_{2}$, which requires accounting for the spin-orbit interaction effect, an ECS approach was proposed for the pure Hund's case $b$ in [317]. It was then empirically improved (by using other ECS parameters and a modified sum rule) and applied to the A-band in [318]. Good agreements with high pressure laboratory spectra and measured atmospheric transmissions were obtained. The same approach enabled an accurate description of LM effects in the $\mathrm{O}_{2}$ rotational band around $60 \mathrm{GHz}$ [319], a region also modeled using the memory function formalism [320]. Many of the abovementioned studies present comparisons between measured and computed spectra at various temperatures, roughly between $200 \mathrm{~K}$ and $300 \mathrm{~K}$. These show that the quality of predictions is generally within $5 \%$ or less and does not significantly change with temperature.

In addition to these applications of previously published theories, a new approach has been proposed for LM between $M$ components. In other words, line-mixing was considered among lines not only defined by the total rotational quantum number $J$ only, but by both the value of $J$ and that of $M$ (magnetic quantum number) that quantifies the projection of $\vec{J}$ on the space-fixed axis. As it is well known, the lines associated with the same $J$ but different $M$ values can be spectrally separated by an electric field and the Stark effect. Starting from [321], an adapted IOS model was developed in [322] and applied to spectra of $\mathrm{CH}_{3} \mathrm{~F}$ with lines separated through the Stark effect. This revealed, with good agreement with measured spectra, the overly simplified approximation made in [323] where discrepancies between measurements and calculations were obtained. Similarly, a model based on the IOS approximation and the developments in [324] was proposed [325] and successfully applied to line-broadening and -mixing within nuclear spin hyperfine components of He-broadened lines of $\mathrm{HI}$ at room temperature. From a purely theoretical point of view, Buffa and Tarrini have corrected [218,326] an error in the method elaborated in [324] which rendered it selfinconsistent. However, as stated in [326], this correction does not change the predicted LM effects and "the equations used by Flaud et al. [325] yield the same line shape as our equations."

\subsubsection{Energy-gap fitting laws and state-to-state cross sections}

Empirical approaches are obviously of interest when more physically based models are not available or too costly to implement. Here, the central idea is to express a given offdiagonal real-valued element of the relaxation matrix in terms of the state-to-state inelastic cross-section connecting the lower (and/or upper) levels of the two interfering lines. This requires switching from the two-dimensional (the two connected states) space of the state-tostate rates to the four-dimensional (the two lower states of the lines and the branches to which the lines belong) space of the absorption lines, an operation achieved by introducing a $(\mathrm{P}, \mathrm{Q}$, $\mathrm{R})$ branch-dependent empirical scaling factor. Considering the state-to-state rates, they are often modeled using energy-gap fitting laws (IV.3.2 of [1] and [327]) based on a few parameters that are generally deduced from fits of line-broadening data. New applications of this type of approach to LM effects for a variety of molecules can be found in [238,245,272,304-308,315,328-332]. Note that there is a rarely used alternative solution. Indeed, several computer codes exist, from semi-classical up to $\mathrm{CC}$, to calculate these stateto-state rates, which can therefore be directly introduced to the LM model (or compared to the fitted ones). Examples are given in IV.3.4f of [1] and [333,334]. This remark also applies to the ECS model where the so-called basic rates can be calculated (eg [335,336]) instead of being modeled with parameters obtained by fits to measured data. 


\subsubsection{The ovoid sphere and hard collision models}

Despite the simplicity of the classical ovoid sphere model ([337] and Sec. IV.3.1b of [1]), this approach, in which an effective collision frequency is empirically adjusted for agreement with measured line widths, provided an accurate description (comparable with the results obtained with the ECS model) of LM in spectra of OCS diluted in He at elevated pressures [316]. It was also applied to the $\mathrm{A}$ band of $\mathrm{O}_{2}$ in [338,339]. Similar remarks can be made for the application of the extended strong collision model ([340] and IV.3.1a of [1]) to the $2 v_{3}$ isotropic Raman band and depolarized Raman spectra of $\mathrm{CO}_{2}$ [341,342].

\subsubsection{Kochanov's approach}

A line-profile model was recently developed by Kochanov, allowing comparison of the influences of the collisional and Doppler broadenings, Dicke narrowing, velocity dependence of the widths and shifts as well as of the LM process [190,343]. The latter appears through a speed-independent first-order LM coefficient [344]. The resulting model involves many parameters likely to be fitted to experiments but, to the best of our knowledge, its ability to accurately describe measurements has not been proven so far.

\subsection{Far wings and associated continua}

We consider here, as in Chapt. V of [1], the spectral regions significantly far from line centers where the absorption manifests as a continuum. We here restrict ourselves to those to which the far wings of monomer lines make a significant contribution, the continua caused by the transient dipole induced during collisions being considered in Sec. 3.6. As noted in [1], first-principles calculations of the contributions of the far wings of allowed (meaning due to the molecule intrinsic dipole) transitions is an extremely difficult problem because many processes must simultaneously be taken into account: the speed dependent line-broadening and -mixing, the finite duration of collisions and initial correlations. The so-called impact approximation breaks down and models such as those described in the preceding sections cannot be used. Recall ( $c f$ Sec. II.3.4 of [1]) that this approximation assumes that the differences $\left|\omega-\omega_{\mathrm{fi}}\right|$ between the photon frequency and those of the optical transitions of the molecule which make the dominant local contribution to the spectrum are much smaller than the inverse of the duration $\left(\tau_{\mathrm{c}}\right)$ of efficient collisions $\left(\right.$ ie $\left.\left|\omega-\omega_{\mathrm{fi}}\right| \tau_{\mathrm{c}}<<1\right)$. In this case, intermolecular collisions can be considered as instantaneous (impact) and the spectrum is not sensitive to details of what happens during collisions but only to the difference between the molecular state before and after a collision. This simplification can thus be used not to far away from line centers. Various approaches have been proposed, those published before 2007 being described in Chapt. V of [1]. From the theoretical point of view, little progress has been made since that time, with the exception of the approach described in Sec. 3.5.1. For the empirical models that rely on adjustments to measured spectra, the main advances concern the $\mathrm{H}_{2} \mathrm{O}$ continuum (Sec. 3.5.4).

\subsubsection{Direct predictions from Classical Molecular Dynamics Simulations}

In [345], CMDS were carried for pure $\mathrm{CO}_{2}$. These provide (cf Secs. 3.2.1 and 3.4.1) the center-of-mass positions and orientations of many (rigid) linear molecules interacting though an input anisotropic potential. For parallel bands, the allowed-dipole vector $\vec{\mu}^{A}$ is along the molecule axis. Its auto-correlation function (ACF) is thus directly calculated from the CMDS, after a proper spectroscopic scaling factor [345] and the choice of time $t=0$ in the calculations. The spectrum is then obtained from the Fourier-Laplace transform of the ACF (ie multiplying the ACF by $\exp (-i \omega t)$ and integrating from $t=0$ to $+\infty)$. A comparison was made [322] 
between the resulting predictions, free of any scaling factor deduced from experiments, and measurements in the wings of the $v_{3}$ band of $\mathrm{CO}_{2}$ at $200 \mathrm{~K}$ and $296 \mathrm{~K}$. A good (in the $\log$ scale used for the comparison) agreement was obtained at $296 \mathrm{~K}$ that degrades slightly at lower temperature. Errors locally reach a factor of two but on a quantity that varies over almost four orders of magnitude ( $c f$ Fig. 14). Shortly thereafter, the contribution of the collision-induced dipole $\vec{\mu}^{I}$ was introduced [346] (calculated as in [347], cf Sec. 3.6.4) and the ACF of the full dipole (including the allowed and induced components) was computed. The results agree quite well with measurements, as illustrated by Fig. 14, and demonstrate that the cross-term $\mu^{A}(0) \cdot \mu^{I}(t)+\mu^{I}(0) \cdot \mu^{A}(t)$ in the ACF makes a significant contribution. Note that similar results were very recently obtained in the same region of $\mathrm{CO}_{2}$ absorption but in the case of collisions with water vapor [348]. Finally, the approach was also applied to the wing of the depolarized Rayleigh roto-translational spectrum of pure $\mathrm{CO}_{2}$ in [346].

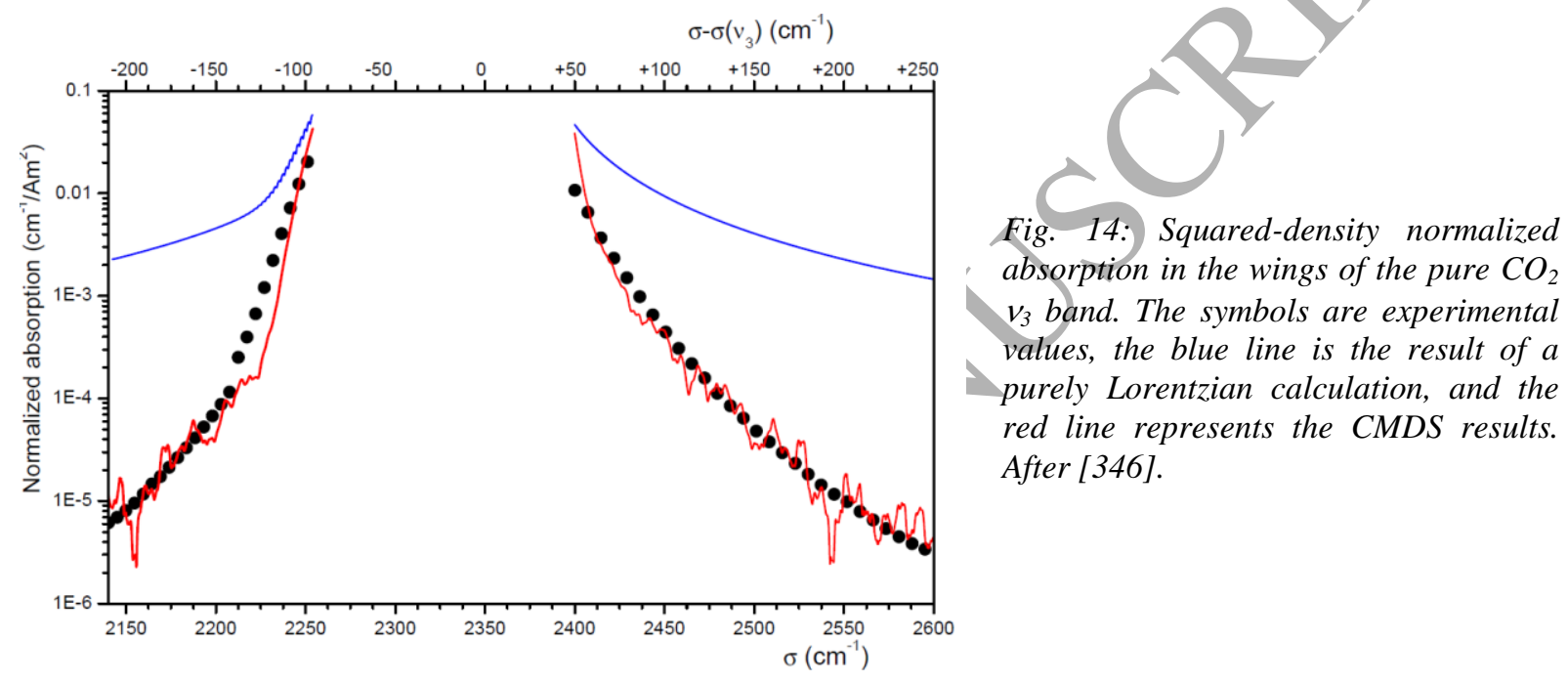

The same type of approach, limited to the contribution of the allowed dipole but improved through a requantization of the CMDS ([121] and Sec. 3.2.1) was applied [124] to spectra of $\mathrm{HCl}$ in $\mathrm{Ar}$ at room temperature. These direct calculations of the entire spectrum show that the absorption in the troughs between lines is super-Lorentzian in the central part of the bands and becomes sub-Lorentzian when going toward the bands wings. Super- (resp. sub-) Lorentzian here mean that the absorption is greater (resp. lower) than that calculated by summing the contributions of all lines with a Lorentz profile. Their analysis demonstrates that this behavior is intimately connected to the evolution of the dipole ACF at very short times (finite duration of collisions) and to the increasing contribution of LM when going away from the band center. Note that the computed values of the relative deviation from the purely Lorentzian behavior agree, within experimental uncertainties, with those deduced from measurements, and that this is achieved without use of any adjustment.

\subsubsection{Non Markovian Energy-Corrected Sudden approach}

A non-Markovian extension of the Energy-Corrected Sudden model for the construction of the relaxation matrix was proposed in [302,349] (see also V.5 of [1]). It goes beyond the impact (Markovian) approximation by taking into account the fact that, in the wings, the energy defect associated with the difference between the frequency of the photon and those of the resonant molecular transitions cannot be neglected. As discussed therein, it enables, in principle, a better description of the far wings than the "usual" ECS model (Sec. 3.4.5) which fails because the impact approximation breaks down. This approach was recently applied to 
$\mathrm{CO}_{2}$ anisotropic Raman scattering spectra [350], showing a good agreement with measurements in the central regions of the bands but a significant underestimation in the wings which is consistent with the previous studies.

\subsubsection{Asymptotic line shape and $\chi$-factor empirical models}

An asymptotic approach for the description of the far line wings was proposed in [351] and applied to $\mathrm{CO}_{2}$ [352] and to the water-vapor continuum in several spectral regions $[351,353,354]$. Good agreement with measurements was obtained at various temperatures, but this required the ad hoc adjustment of some parameters. The proposed model thus has limited predictive capabilities and it should be considered as empirical, as the $\chi$-factor approach of [311,355,356] (see also V.2 of [1]) and the MT_CKD continuum discussed below.

\subsubsection{The MT_CKD water-vapor continuum}

The absorption spectrum of water vapor contains not only narrow rovibrational lines whose spectroscopic parameters are tabulated in databases but also a relatively weak underlying broadband continuum with a slow frequency dependence. In the Earth's atmosphere, this continuum (see also Sec. 4.3.1, [357] and appendix V.A of [1]) can be decomposed into a "self" component due to water-water intermolecular interactions and a "foreign" component due to water-nitrogen and water-oxygen interactions. According to the usual definition, these continua are obtained after subtraction, from the total absorption coefficient, of the contribution of the $\mathrm{H}_{2} \mathrm{O}$ lines calculated using Voigt profiles truncated at $\pm 25 \mathrm{~cm}^{-1}$ from their centers [358]. For a binary mixture of water vapor and nitrogen, for instance, the quantity obtained after this exercise has a component that increases quadratically with the water partial pressure and another one proportional to the product of the $\mathrm{H}_{2} \mathrm{O}$ and $\mathrm{N}_{2}$ partial pressures. These are the so-called self- and foreign-continuum contributions $\left(\alpha_{\mathrm{S}}^{\mathrm{C}}\right.$ and $\alpha_{\mathrm{F}}^{\mathrm{C}}$ ) to the total absorption coefficient $\alpha_{\mathrm{T}}^{\mathrm{C}}$, respectively. The latter can thus be written in terms of the associated normalized absorption cross sections $C_{\mathrm{s}}(T)$ and $C_{\mathrm{f}}(T)$ (often expressed in $\mathrm{cm}^{2}$ molecule ${ }^{-1} \mathrm{~atm}^{-1}$ ) through:

$\alpha_{\mathrm{T}}^{\mathrm{C}}\left(v, T, P_{H 2 O}, P_{N 2}\right)=\alpha_{\mathrm{S}}^{\mathrm{C}}\left(v, T, P_{H 2 O}, P_{\mathrm{N} 2}\right)+\alpha_{\mathrm{F}}^{\mathrm{C}}\left(v, T, P_{H 2 O}, P_{N 2}\right)=\frac{1}{k_{B} T}\left[C_{\mathrm{S}}(v, T) P_{H 2 O}^{2}+C_{\mathrm{F}}(v, T) P_{H 2 O} P_{N 2}\right]$, where $k_{B}$ is the Boltzmann constant.

The default description of the normalized continua $C_{\mathrm{S}}(T)$ an $C_{\mathrm{F}}(T)$ of the preceding equation has generally been the pragmatic approach of Clough-Kneizys-Davies (CKD) [358] and its successor Mlawer-Tobin-CKD (MT_CKD) [359,360]). It is a semi-empirical formulation based on ad hoc (line-profile) parameters adjusted to measured spectra. The latter consist mainly of laboratory (eg [361-364], see [357]) and field (eg [365]) measurements in spectral regions that have [366,367] the strongest impact on the radiative fluxes in the Earth atmosphere. The $10 \mu \mathrm{m}$ window has been studied for decades (eg [357,362,368-370]) and a strong constraint of the MT_CKD self continuum (ie pure $\mathrm{H}_{2} \mathrm{O}$ ) in this region comes from field measurements (eg [371]). In the mid- and near-infrared regions, the MT_CKD continuum is mostly an extrapolation, which up to recently was lacking experimental validation. In recent years, considerable experimental efforts have been devoted to this issue as discussed in Sec. 4.3.1. The recent changes made to the MT_CKD continua are described in $[359,360]$ and the current versions for both the self $\left(\mathrm{H}_{2} \mathrm{O}-\mathrm{H}_{2} \mathrm{O}\right)$ and foreign $\left(\mathrm{H}_{2} \mathrm{O}\right.$-air) contributions can be downloaded at [372] where more information on the continua is given. A comparison between several sets of measurements of the self-continuum and the last version (V3.0) of the MT_CKD model is plotted in Fig. 15. If one excludes the FTS experiments from CAVIAR and Tomsk [373-375], which are in striking disagreement (as further discussed in Sec. 4.3.1a), the MT_CKD3.0 general evolution is well supported by recent CRDS and OF- 
CEAS measurements [376-381], with very good agreement in the $1.6 \mu \mathrm{m}$ and $1.25 \mu \mathrm{m}$ windows but significant deviations at the center of the $2.1 \mu \mathrm{m}$ and $4.0 \mu \mathrm{m}$ windows.

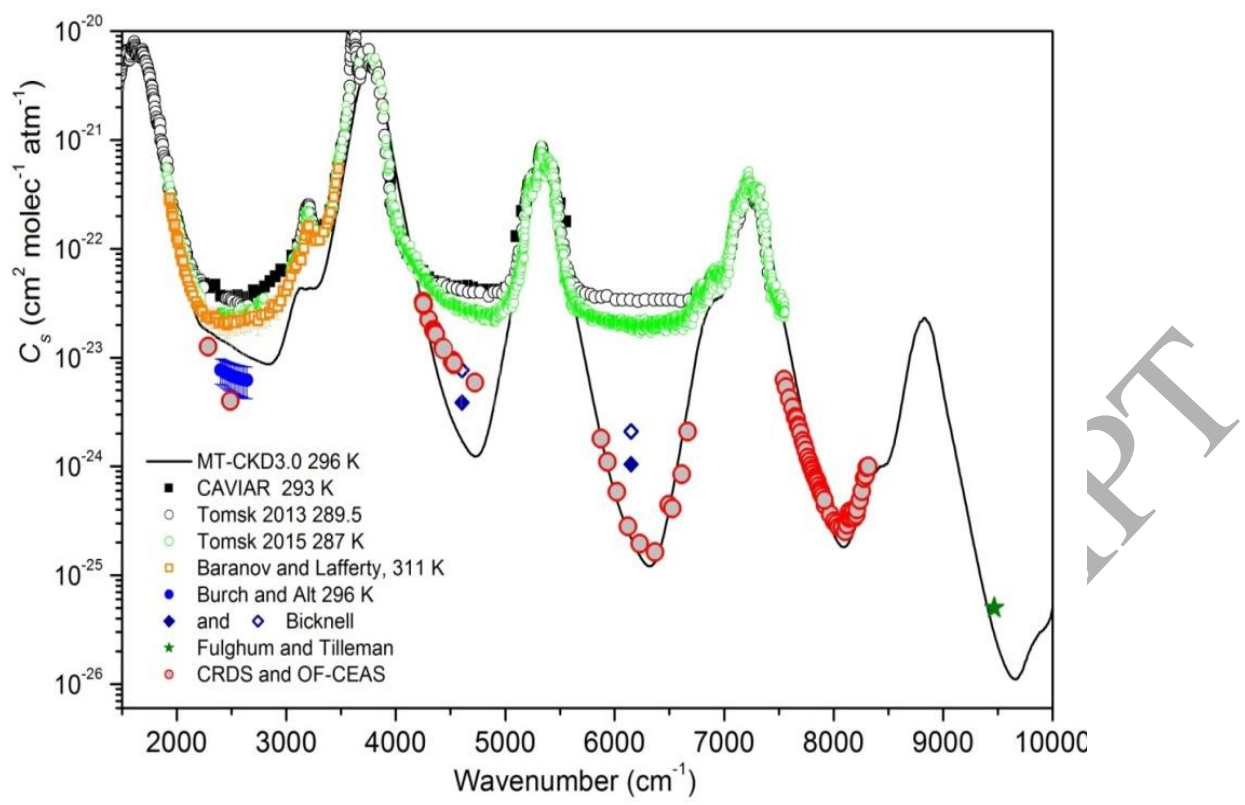

Fig. 15: Comparison between the MT_CKD(v3.0) self-continuum cross section $\mathrm{C}_{s}$ of $\mathrm{H}_{2} \mathrm{O}$ at room temperature (line) and measurements with grating spectrograph [362] (light blue circles); by calorimetric interferometry [382] (full and open blue diamonds) [383] (green star) ; by FTS from [373] (blue squares), [384] (orange open squares), [374] and [375] (open grey and light green circles); by CRDS and OF-CEAS from [376-381] (open red circles).

The origin of the temperature dependence of the water continuum in the MT_CKD model is detailed in [359]. In particular, it is indicated that the MT_CKD temperature dependence of the self continuum relies on the measurements by Burch et al. in the 700-1200 $\mathrm{cm}^{-1}$ and 2400-2800 $\mathrm{cm}^{-1}$ windows [361-363, 385]. To the best of our knowledge, the much more recent results of [384] were not taken into account to adjust the MT_CKD model. For the foreign continuum, the MT_CKD values are practically independent of temperature in the infrared, consistently with what was experimentally found [386-388].

\subsection{Collision-induced absorption (CIA)}

We consider here CIA where it is the transient dipole created during the interaction of molecular pairs that leads to light-matter interaction and produces a spectrum, as discussed in [389,390] and Chapt. VI of [1]. Note that the terms interaction-induced, or supermolecular absorption may also be used, and it is sometimes more appropriate since dimers may also contribute to the absorption. A discussion on the definition of CIA in the context of the water vapor continuum can be found in [391]. We focus below on theoretical treatments of CIA involving molecules, ie not rare-gas mixtures, and in general disregard collision-induced light scattering. We begin with the state-of-the-art coupled quantum scattering treatments in Sec. 3.6.1. After that comes a brief presentation of new findings in intra-collisional interference, a phenomenon where a coupled scattering-treatment is needed (Sec. 3.6.2). The "standard" binary-collision treatment, which normally entails one-dimensional quantum scattering, and sometimes classical two-body collision calculations, is covered in Sec. 3.6.3. Progress in classical many-body treatments, so-called molecular dynamics simulations, is described in Sec. 3.6.4. Finally, integrated spectra, or spectral moments, are discussed in Sec. 3.6.5.

\subsubsection{Quantum scattering advances}


Refined quantum-dynamical calculations, based on the CC method and including the full anisotropic potential, were made in [392] for CIA spectra of $\mathrm{H}_{2}$. The free-free dipole matrix elements are obtained on-the-fly, which gives this new method an advantage by comparison to a previous study [393] that also included the anisotropy of the interaction potential in CIA calculations. The more challenging case of nitrogen [394] was then considered and, since the $\mathrm{N}_{2}-\mathrm{N}_{2}$ pair potential supports many bound (dimer) states, the dimer contribution to the absorption was also taken into account. This enabled quantification of the various contributions to the spectrum, as illustrated by Fig. 16. Full $\mathrm{CC}$ calculations of $\mathrm{N}_{2}$ CIA, with an anisotropic potential, are barely feasible. Nevertheless, the authors performed one such benchmark calculation, at low energy and for one representative photon frequency, which was compared with CS calculations. They concluded that the latter capture most of the effect from the anisotropy of the potential, and thus the CS approximation was applied in the final calculations of absorption coefficients. The studies [392] and [394] both include comparisons with measurements at temperatures ranging from $77 \mathrm{~K}$ to $300 \mathrm{~K}$. While excellent agreement is obtained above $\sim 150 \mathrm{~K}$, the calculations increasingly underestimate the peak absorption with decreasing temperature (by about $20 \%$ at $78 \mathrm{~K}$ [394]).

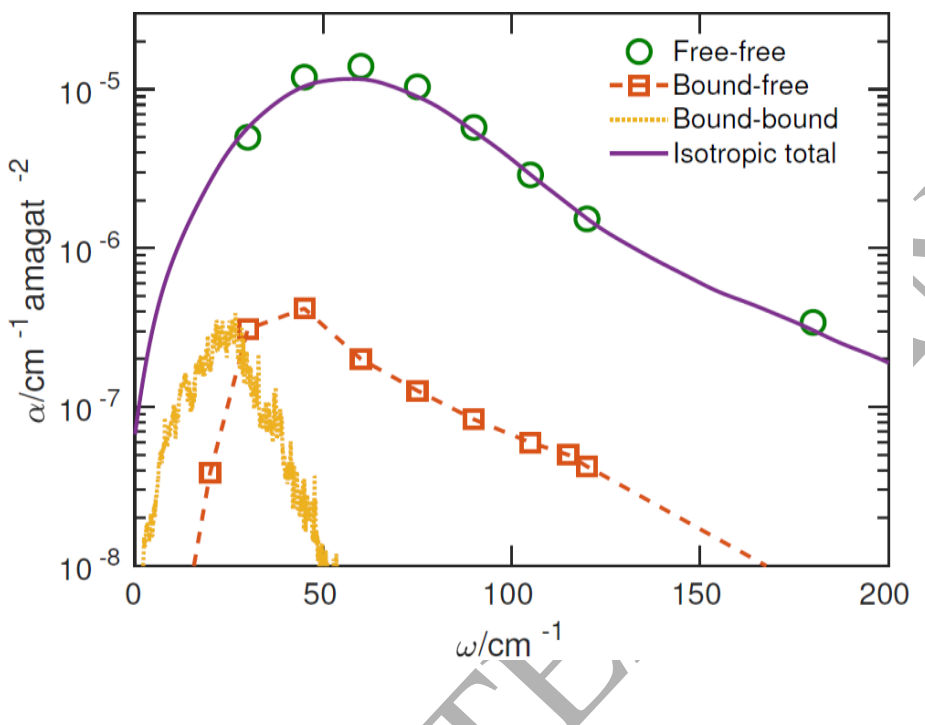

Fig. 16: Different contributions to the squared-density normalized CIA in the rototranslational band of pure $N_{2}$ at $T=78 \mathrm{~K}$ computed using the full anisotropic potential in the CS approximation. The full line denotes the (smoothed for clarity) total absorption obtained in the isotropic approximation. Courtesy of T. Karman, after [394].

From the same group as the works described above come remarkable studies of the spinforbidden A-band transition in $\mathrm{O}_{2}$, induced by collisions with a second oxygen [395] or a nitrogen [396] molecule. For the former work, new dipole and potential energy surfaces were computed [397]. In the comparison of $\mathrm{O}_{2}$ and $\mathrm{N}_{2}$ as a collisional partner the authors conclude that there are large differences between the two, in agreement with measurements, but contrary to what has previously been believed [389]. The reason is that an exchange mechanism, which is allowed for $\mathrm{O}_{2}-\mathrm{O}_{2}$ but not for $\mathrm{N}_{2}-\mathrm{O}_{2}$, is responsible for much of the absorption in pure oxygen. In a comparison of the computed and measured absorption spectra the absolute absorptions agree within an order of magnitude and, through scaling of the various contributions, good agreements between for both the magnitudes and shapes of measured and calculated spectra are obtained.

\subsubsection{Intra-collisional interference effects}

CIA provides a convenient case for the study of intra-collisional effects, a phenomenon reviewed in [398]. In systems like HD, which has a rather small dipole moment, interference can be observed between the permanent and the interaction-induced dipoles ( $c f$ VI.6.2 of [1] and references therein). An equivalent mechanism, the interference of Raman-allowed S-lines 
and the collision-induced light scattering, has been studied in pure $\mathrm{H}_{2}$ [399] and in a $\mathrm{H}_{2}-\mathrm{Xe}$ mixture [400].

\subsubsection{Standard binary-collision treatments}

Frommhold and co-workers have made several computational studies of CIA in hydrogen-containing gases, at temperatures from hundreds to thousands of $\mathrm{K}$. These works are of importance for further studies of some stellar atmospheres where the level of ionization is low, such as those of cool white dwarfs. Their method is usually quantum mechanical, but ignores anisotropic components of the intermolecular potential ("isotropic approximation"). Investigations were made for pure $\mathrm{H}_{2}$ [401-403] and for $\mathrm{H}_{2}$ with $\mathrm{He}$ [404], with corresponding studies for deuterium and tritium in [405-407]. Most of those studies include comparisons with laboratory measurements at several different temperatures, and they are done using recent $a b$ initio potential and dipole data. The agreement with measurements is generally considered as very good, at least in the log-scales used for comparisons of absorption coefficients which vary by several orders of magnitude throughout the considered spectral range. El-Kader and coworkers pursued studies at a similar level of theory for $\mathrm{H}_{2}-(\mathrm{Ar}, \mathrm{He})$ [408,409], $\mathrm{CF}_{4}-\mathrm{He}$ mixtures [410], pure methane [411], pure $\mathrm{N}_{2}$ [412], and pure $\mathrm{O}_{2}$ [413]. A classical trajectory study of CIA in $\mathrm{CO}_{2}-(\mathrm{Ar}, \mathrm{Xe})$ mixtures can be found in [414].

\subsubsection{Direct predictions from Classical Molecular Dynamics Simulations}

The CMDS proposed in [415] for the far infrared CIA by $\mathrm{CO}_{2}$ was revisited in [347] which demonstrated the importance of vibrational and back-induction contributions. Taking these effects into account largely obviated the need for the ad hoc short-range overlap contributions empirically introduced in [415]. Subsequently, this approach enabled the first analysis [346] of the respective contributions of allowed and induced dipoles, and the socalled intra-collisional interference, in the wings of the $\mathrm{CO}_{2} v_{3}$ band (Sec. 3.5.1). The predictions of the far infrared roto-translational CIA band of $\mathrm{N}_{2}$ [416] were, at the time, the first in which the spectral effects of the intermolecular anisotropy and of (ab initio) shortrange dipole components were studied for $\mathrm{N}_{2}$. Since then, much more refined computations have been made [394] (Sec. 3.6.1). CMDS also enabled the modeling of CIA in the region of the first overtone of $\mathrm{N}_{2}$ with the analysis (Fig. 17) of the spectral contributions of the single and double (or simultaneous) transitions [417]. The comparisons with measurements made in [417] also show that, while the CMDS slightly overestimate absorption at room temperature, they strongly underestimate $(\sim 40 \%)$ it around $90 \mathrm{~K}$. Note that CMDS were very recently made for the fundamental band of $\mathrm{N}_{2}$ colliding with $\mathrm{H}_{2} \mathrm{O}$ [348]. Agreement within the uncertainties of measurements [418] was obtained and the calculations confirm the considerable enhancement of the peak value and width of the CIA spectrum for $\mathrm{N}_{2}-\mathrm{H}_{2} \mathrm{O}$ collisions with respect to those for pure $\mathrm{N}_{2}$. 


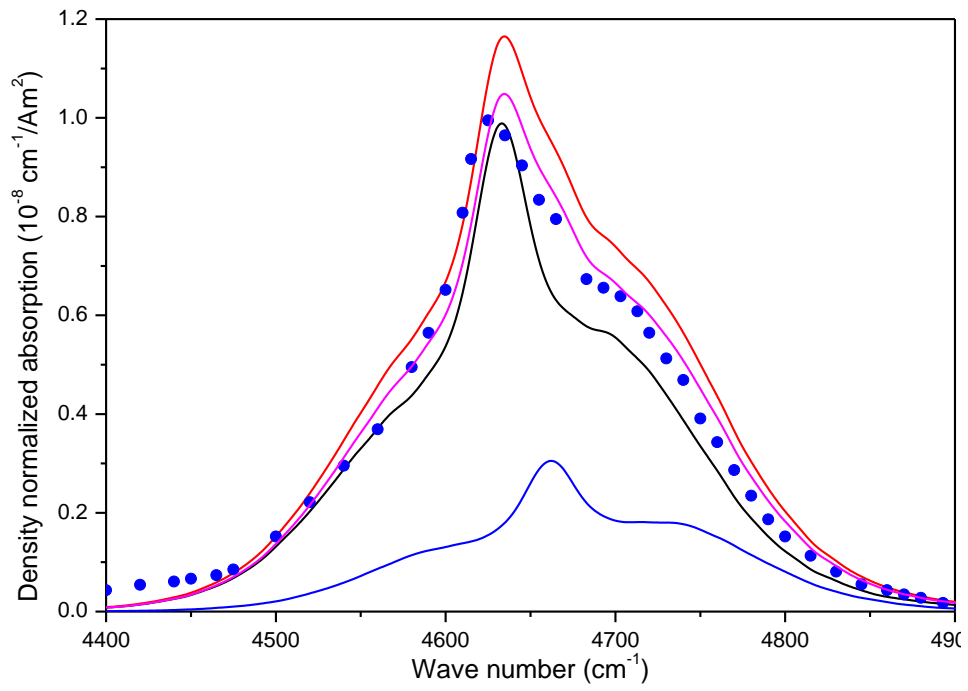

3.6.5 Integrated CIA intensities

CIA may be studied theoretically without computing spectra, but rather integrated spectra or so-called spectral moments. These can in general be calculated with less effort using sum formulas [389], and may be used in combination with line-shape models (eg $[420,421])$ to provide spectra. Recent studies of spectral moments include those in the $\mathrm{N}_{2}$ fundamental for pure $\mathrm{N}_{2}$ [422] and water-nitrogen [418], pure methane [423], methane-argon mixtures [424] and $\mathrm{CO}_{2}$ (pure and with rare gases) [425]. In all those studies, new potential and/or dipole data were also developed. Predictions generally agree with measurements within typically $10 \%$ and correctly describe the influence of temperature (eg $[422,425])$. Spectral moments for $\mathrm{CO}_{2}$ have been computed in [426] where formulas including the anisotropy of the potential are given and the importance of the vibrational dependence of the polarizability is established. In a study of water vapor [427], spectral moments were computed and used in combination with the line shape of [420] to produce CIA spectra. Finally, expressions for spectral moments which can be used to separate bound-state contributions from the free-to-free component have been derived $[424,428]$.

\section{Available data}

This section proposes a number of references in which data are provided (either directly or on request from the authors) that can be used in order to calculate absorption spectra. The associated experimental or calculated results enable simulations of isolated-line shapes (Sec. 4.1), of line-mixing effects (Sec. 4.2), of the far wings and associated continua (Sec. 4.3), and of collision-induced absorption (Sec. 4.4). Although the tables in these sections may not be exhaustive, they give numerous starting points for bibliographical searches, while data and other references are also given in [1,244,429-433]. Finally, the recent evolution of spectroscopic databases is discussed in Sec. 4.5.

For the extraction of spectral-shape parameters from measured spectra, several sophisticated fitting programs have been developed (eg [99,170,434]. It here is important to recall the importance, when the model involves many parameters, of using least-squares fitting methods where parameters are simultaneously adjusted to fit multiple spectra. Good examples in which multispectrum fitting methods have been used to retrieve many spectral parameters can be found in $[9,100,168,435-437]$ for instance. Furthermore, when (over)simplified fitting profiles and/or noisy experiments are used, one should very carefully consider the overall uncertainty on the retrieved parameters, including those due to both the model approximations and the 
measurement errors and noise. Otherwise hasty conclusions may be drawn, as shown in [438] for instance.

\subsection{Isolated lines}

The bibliography providing isolated-line shape parameters deduced from laboratory measurements is much too vast to be exhaustively reviewed, even when restricted to the publications of the decade. We have thus decided to only retain, in Table 3, those in which line profiles more refined than the VP were used. Readers searching for pressure-broadening and -shifting coefficients adapted to the VP can consult the broad bibliography provided with spectroscopic databases [430,431,439]. It is important to note that most of the studies cited below are limited to room temperature. Furthermore, many of those in which various temperatures were investigated used fits with "simple" non-Voigt profiles such as the SC, HC and qSDV. Only a few pioneers [103,153,181,440] have tested sophisticated line-shape models including the HTP.

\begin{tabular}{|c|c|c|c|c|}
\hline Molec & Region & Perturber & Model & Refs. \\
\hline \multirow{14}{*}{$\mathrm{H}_{2} \mathrm{O}$} & $0.81-0.83 \mu \mathrm{m}$ & $\mathrm{H}_{2} \mathrm{O}$, air & $\mathrm{HC}, \mathrm{qSDV}$ & {$[284]$} \\
\hline & $0.93 \mu \mathrm{m}$ & $\begin{array}{l}\mathrm{N}_{2}, \text { air } \\
\mathrm{H}_{2} \mathrm{O}, \mathrm{N}_{2} \\
\mathrm{~N}_{2}, \mathrm{SF}_{6}\end{array}$ & $\begin{array}{l}\text { SC,HC,qSDV } \\
\text { SC } \\
\text { HT }\end{array}$ & $\begin{array}{l}{[441]} \\
{[442]} \\
{[188]}\end{array}$ \\
\hline & $1.28 \mu \mathrm{m}$ & $\mathrm{H}_{2} \mathrm{O}$ & SC,HC,qSDV,qSDHC,HT,pChSDHC & [8] \\
\hline & $1.39 \mu \mathrm{m}$ & $\begin{array}{l}\mathrm{H}_{2} \mathrm{O} \\
\mathrm{H}_{2} \mathrm{O} \\
\mathrm{N}_{2}\end{array}$ & $\begin{array}{l}\text { pChSDHC,HT } \\
\text { SC,hSDV,hSDSC } \\
\text { SC,qSDV,qSDHC }\end{array}$ & $\begin{array}{l}{[189]} \\
{[93]} \\
{[435]}\end{array}$ \\
\hline & $1.4 \mu \mathrm{m}$ & $\begin{array}{l}\mathrm{H}_{2} \mathrm{O}, \mathrm{Ar} \\
\mathrm{H}_{2} \mathrm{O}, \mathrm{N}_{2} \\
\mathrm{H}_{2} \mathrm{O}, \mathrm{N}_{2} \\
\mathrm{H}_{2} \mathrm{O}, \mathrm{N}_{2}, \mathrm{He} \\
\mathrm{He}\end{array}$ & $\begin{array}{l}\text { SC } \\
\text { SC,HC,qSDV } \\
\text { SC,qSDHC } \\
\text { SC,qSDV } \\
\text { SC }\end{array}$ & $\begin{array}{l}{\left[185^{*}\right]} \\
{\left[182^{*}\right]} \\
{[443]} \\
{[444]} \\
{[445]}\end{array}$ \\
\hline & $1.39-1.47 \mu \mathrm{m}$ & $\mathrm{H}_{2} \mathrm{O}$ & $\mathrm{HC}, \mathrm{qSDV}$ & {$[446 *]$} \\
\hline & $1.6 \mu \mathrm{m}$ & $\mathrm{H}_{2} \mathrm{O}$ & HT & {$[188]$} \\
\hline & $2.5 \mu \mathrm{m}$ & $\mathrm{H}_{2} \mathrm{O}, \mathrm{CO}_{2}$, & SC & {$[186 *]$} \\
\hline & $2.6 \mu \mathrm{m}$ & $\mathrm{N}_{2}$ & HT & {$[143,188]$} \\
\hline & $2.5-2.9 \mu \mathrm{m}$ & air & qSDV & {$[282 *]$} \\
\hline & $2.5-5.4 \mu \mathrm{m}$ & $\mathrm{H}_{2} \mathrm{O}$, air & qSDHC & [440*] \\
\hline & $2.5-5.4 \mu \mathrm{m}$ & $\mathrm{H}_{2} \mathrm{O} Y$ & SDV & {$[447]$} \\
\hline & $2.7,6.25 \mu \mathrm{m}$ & $\mathrm{H}_{2} \mathrm{O}$ & $\mathrm{HC}$ & [448] \\
\hline & \begin{tabular}{|l|}
$22 \mathrm{GHz}$ \\
\end{tabular} & $\mathrm{H}_{2} \mathrm{O}$, air & SDV & [449] \\
\hline HDO & $\begin{array}{l}2.7 \mu \mathrm{m} \\
3.6 \mu \mathrm{m} \\
7.4 \mu \mathrm{m}\end{array}$ & $\begin{array}{l}\mathrm{CO}_{2}, \mathrm{HDO} \\
\mathrm{CO}_{2}, \mathrm{HDO} \\
\mathrm{CO}_{2}\end{array}$ & qSDV & $\begin{array}{l}{\left[286^{*}\right]} \\
{\left[285^{*}\right]} \\
{[281 *]}\end{array}$ \\
\hline $\mathrm{HCl}$ & $3.5 \mu \mathrm{m}$ & $\begin{array}{l}\mathrm{N}_{2}, \mathrm{He}, \mathrm{Ar}, \mathrm{Xe} \\
\mathrm{Ar}\end{array}$ & $\begin{array}{l}\text { SC,HC,RG } \\
\text { qSDHC }\end{array}$ & $\begin{array}{l}{[450]} \\
{[121]} \\
\end{array}$ \\
\hline \multirow{5}{*}{$\mathrm{CH}_{4}^{\prime}$} & $7.7 \mu \mathrm{m}$ & 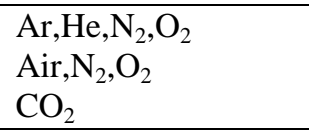 & $\begin{array}{l}\text { SC,HC,qSDV,qSDHC,HT } \\
\text { SC,HC } \\
\text { SC,HC }\end{array}$ & $\begin{array}{l}{[451]} \\
{[452]} \\
{[453]}\end{array}$ \\
\hline & $3.3 \mu \mathrm{m}$ & $\mathrm{Ar}, \mathrm{He}, \mathrm{H}_{2}, \mathrm{~N}_{2}, \mathrm{CO}_{2}, \mathrm{O}_{2}$ & $\mathrm{SC}$ & [454] \\
\hline & $1.7 \mu \mathrm{m}$ & $\begin{array}{l}\mathrm{CH}_{4} \text {,air } \\
\text { air }\end{array}$ & $\begin{array}{l}\text { qSDV } \\
\text { HT }\end{array}$ & $\begin{array}{l}{\left[102^{*}, 455^{*}\right]} \\
{[9]}\end{array}$ \\
\hline & $2.2 \mu \mathrm{m}$ & $\mathrm{CH}_{4}$, air & qSDV & [456] \\
\hline & $1.6 \mu \mathrm{m}$ & $\begin{array}{l}\mathrm{CO}_{2} \text {,air } \\
\mathrm{CO}_{2} \\
\text { Air } \\
\text { Air } \\
\text { Air }\end{array}$ & $\begin{array}{l}\mathrm{qSDV} \\
\mathrm{HC}, \mathrm{qSDV}, \mathrm{qSDHC}, \mathrm{HT} \\
\text { SC,HC,qSDV,qSDHC } \\
\text { qSDV,qSDHC } \\
\text { HT }\end{array}$ & $\begin{array}{l}{[457]} \\
{[458]} \\
{[459]} \\
{[181 *]} \\
{[12,460]}\end{array}$ \\
\hline
\end{tabular}




\begin{tabular}{|c|c|c|c|c|}
\hline \multirow[t]{5}{*}{$\mathrm{CO}_{2}$} & & $\begin{array}{l}\mathrm{O}_{2} \\
\mathrm{CO}_{2} \\
\mathrm{CO}_{2}\end{array}$ & $\begin{array}{l}\mathrm{qSDV} \\
\text { SC } \\
\mathrm{qSDV}\end{array}$ & $\begin{array}{l}{[461 *]} \\
{[462]} \\
{[463]}\end{array}$ \\
\hline & $2.0 \mu \mathrm{m}$ & $\begin{array}{l}\mathrm{CO}_{2} \\
\mathrm{CO}_{2} \\
\mathrm{Air} \\
\mathrm{Air}, \mathrm{CO}_{2} \\
\end{array}$ & $\begin{array}{l}\text { SC,HC,cSC,cHC,hSDV } \\
\text { HT } \\
\text { SC,HC,qSDV,qSDHC,HT } \\
\text { qSDV }\end{array}$ & $\begin{array}{l}{[464]} \\
{[60]} \\
{[465]} \\
{[466 *]}\end{array}$ \\
\hline & $3.0 \mu \mathrm{m}$ & $\mathrm{CO}_{2}$ & HC,qSDV & [313] \\
\hline & $4.3 \mu \mathrm{m}$ & $\mathrm{N}_{2}$ & qSDHC & {$[103 *]$} \\
\hline & $10.0 \mu \mathrm{m}$ & $\mathrm{CO}_{2}$ & $\mathrm{HC}$ & [467] \\
\hline \multirow[t]{2}{*}{$\mathrm{NH}_{3}$} & $9.1 \mu \mathrm{m}$ & $\mathrm{N}_{2}, \mathrm{O}_{2}, \mathrm{CO}_{2}, \mathrm{H}_{2} \mathrm{O}$ & $\mathrm{SC}$ & [468] \\
\hline & $10 \mu \mathrm{m}$ & $\mathrm{NH}_{3}$ & SC,HC,hSDV & [469] \\
\hline $\mathrm{N}_{2} \mathrm{O}$ & $4.5 \mu \mathrm{m}$ & air & qSDV & [470] \\
\hline $\mathrm{H}_{2}$ & $0.8,1.3,2.1 \mu \mathrm{m}$ & $\mathrm{H}_{2}$ & HT & {$[153 *]$} \\
\hline $\mathrm{CH}_{3} \mathrm{Br}$ & $6.9 \mu \mathrm{m}$ & $\mathrm{CH}_{3} \mathrm{Br}, \mathrm{N}_{2}, \mathrm{O}_{2}$ & $\mathrm{SC}$ & [471] \\
\hline $\mathrm{CH}_{3} \mathrm{Cl}$ & $100-800 \mathrm{GHz}$ & $\begin{array}{l}\mathrm{CO}_{2} \\
\mathrm{O}_{2} \\
\mathrm{He}, \mathrm{Ar}, \mathrm{Kr}\end{array}$ & $\begin{array}{l}\text { SC,qSDV } \\
\text { SC,qSDV } \\
\text { SC,qSDV,qSDSC }\end{array}$ & $\begin{array}{l}{[472]} \\
{[280]} \\
{[473]}\end{array}$ \\
\hline $\mathrm{CH}_{2} \mathrm{CHF}$ & $8.7 \mu \mathrm{m}$ & $\mathrm{CH}_{2} \mathrm{CHF}$ & $\mathrm{HC}$ & [434] \\
\hline \multirow{4}{*}{$\mathrm{CO}$} & Rotational & $\mathrm{CO}$ & qSDV & [474] \\
\hline & $4.6 \mu \mathrm{m}$ & $\begin{array}{l}\mathrm{He} \\
\mathrm{Xe}\end{array}$ & $\begin{array}{l}\text { HC,qSDV,qSDHC } \\
\text { HC,SC,qSDSC,hSDHC }\end{array}$ & $\begin{array}{l}{[475]} \\
{[476]}\end{array}$ \\
\hline & $2.3 \mu \mathrm{m}$ & $\begin{array}{l}\mathrm{CO}, \text { air } \\
\text { Air } \\
\mathrm{CO}_{2} \\
\mathrm{CO}, \mathrm{H}_{2}\end{array}$ & $\begin{array}{l}\text { qSDV } \\
\text { qSDV } \\
\text { qSDV,HC } \\
\text { HC,qSDV,qSDHC }\end{array}$ & $\begin{array}{l}{[477 *]} \\
{[478 *]} \\
{[332]} \\
{[238]}\end{array}$ \\
\hline & $1.57 \mu \mathrm{m}$ & $\begin{array}{l}\mathrm{He}, \mathrm{Ar}, \mathrm{Kr}_{,} \mathrm{SF}_{6} \\
\mathrm{CO} \\
\mathrm{Ar} \\
\mathrm{He}\end{array}$ & $\begin{array}{l}\text { HC,SC,qSDV,qSDHC,HT } \\
\text { SC,qSDV } \\
\text { SC,HC,qSDV,qSDSC,pCqSDSC, } \\
\text { qSDHC,HT } \\
\text { qSDHC }\end{array}$ & $\begin{array}{l}{[168]} \\
{[479]} \\
{[480]} \\
{[10]} \\
\end{array}$ \\
\hline $\mathrm{PH}_{3}$ & $4-5 \mu \mathrm{m}$ & $\mathrm{PH}_{3}$ & qSDV & {$[481]$} \\
\hline $\mathrm{CN}$ & $0.9 \mu \mathrm{m}$ & $\mathrm{Ar}$ & SC,qSDV & [482] \\
\hline \multirow{7}{*}{$\mathrm{C}_{2} \mathrm{H}_{2}$} & $7.5 \mu \mathrm{m}$ & $\begin{array}{l}\mathrm{N}_{2} \\
\mathrm{~N}_{2}, \mathrm{~A} \\
\mathrm{O}_{2}\end{array}$ & $\begin{array}{l}\text { HC,SC,SDHC } \\
\text { HC,SC } \\
\text { HC,SC,qSDV }\end{array}$ & $\begin{array}{l}{[483]} \\
{[484]} \\
{[485]}\end{array}$ \\
\hline & $1.5 \mu \mathrm{m}$ & $\begin{array}{l}\mathrm{C}_{2} \mathrm{H}_{2}, \mathrm{~N}_{2} \\
\mathrm{C}_{2} \mathrm{H}_{2} \\
\mathrm{C}_{2} \mathrm{H}_{2}, \mathrm{~N}_{2} \\
\end{array}$ & $\begin{array}{l}\text { HC } \\
\text { HC,SC,RG,hSDV,hSDHC,ChSDHC } \\
\text { hSDV,qSDV } \\
\text { HC,RG,hSDV } \\
\text { HC,qSDV,HT }\end{array}$ & $\begin{array}{l}{\left[235^{*}, 252^{*}\right]} \\
{[486]} \\
{\left[183^{*}\right]} \\
{[487]} \\
{[184 *]} \\
\end{array}$ \\
\hline & $1.5 \mu \mathrm{m}$ & $\mathrm{C}_{2} \mathrm{H}_{2}$ & SDV & {$[330 *]$} \\
\hline & $1.4 \mu \mathrm{m}$ & $\mathrm{C}_{2} \mathrm{H}_{2}$ & HT & [96] \\
\hline & $118 \mathrm{GHz}$ & $\mathrm{O}_{2}$ & qSDV & {$[488]$} \\
\hline & $0.69 \mu \mathrm{m}$ & $\mathrm{O}_{2}, \mathrm{~N}_{2}$ & $\begin{array}{l}\text { SC,HC,qSDV,hSDV,qSDHC,hSDHC, } \\
\text { HT,pChSDHC } \\
\text { qSDV } \\
\text { SC,HC,qSDV,qSDSC,qSDHC } \\
\text { SC,HC,qSDV } \\
\text { SC,HC,hSDV,hSDSC,hSDHC,hSDBB, } \\
\text { hSDB } \\
\text { SC,HC,qSDV,hSDV,qSDSC,hSDSC, } \\
\text { qSDHC,hSDHC }\end{array}$ & $\begin{array}{l}{[187]} \\
{[437]} \\
{[11,489,490]} \\
{[491]} \\
\\
{[149]} \\
\\
{[492]}\end{array}$ \\
\hline & $0.76 \mu \mathrm{m}$ & $\begin{array}{l}\mathrm{O}_{2} \\
\mathrm{~N}_{2} \\
\mathrm{O}_{2}, \text { air }\end{array}$ & $\begin{array}{l}\text { SC,SDV,SDHC } \\
\text { qSDV } \\
\text { SC }\end{array}$ & $\begin{array}{l}{[306]} \\
{[245]} \\
{[493-496]}\end{array}$ \\
\hline
\end{tabular}

Table 3: Bibliography providing data from fits of measured isolated line shapes using various profiles more refined than the usual Voigt or Lorentz ones. Acronyms: "SC": Soft Collisions ; 
"BB": Billiard Ball ; "B": Blackmore, "RG": Rautian-Galatry ;"V": Voigt ; "SD”: Speed Dependent ; $q$ ": quadratic ; $h ":$ hypergeometric ;" $p C$ ": partially Correlated ; "HT": Hartmann-Tran. The references marked with an asterisk (eg [415*]) are those in which the temperature dependence of line-shape parameters other that the usual broadening (and shifting) were investigated.

\subsection{Line mixing}

Table 4 completes tables IV.5 and IV.6 of [1] by giving a list of recent references providing data that enable the inclusion of LM effects in spectra calculations. The available parameters, which can be used as explained in Sec. IV.2 of [1], consist either of relaxationmatrix elements or first-order LM coefficients within the so-called "Rosenkranz approximation" [344].

\begin{tabular}{|c|c|c|c|}
\hline Molec & Region & Techn & Refs. \\
\hline $\mathrm{C}_{2} \mathrm{H}_{2}$ & $1.5 \mu \mathrm{m}$ & $\begin{array}{l}\text { Exp \& Calc } \\
\text { Exp }\end{array}$ & $\begin{array}{l}{[330 *]} \\
{[486]}\end{array}$ \\
\hline $\mathrm{CH}_{3} \mathrm{D}$ & $7.66 \mu \mathrm{m}$ & Exp & {$[259 *]$} \\
\hline $\mathrm{CH}_{4}$ & $\begin{array}{l}1.6 \mu \mathrm{m} \\
2.2 \mu \mathrm{m} \\
3 \mu \mathrm{m} \\
6.4 \mu \mathrm{m} \\
8 \mu \mathrm{m} \\
\end{array}$ & $\begin{array}{l}\text { Exp } \\
\text { Calc } \\
\text { Exp } \\
\text { Exp } \\
\text { Exp } \\
\text { Exp } \\
\end{array}$ & $\begin{array}{l}{\left[9,102 *, 455^{*}, 497,498\right]} \\
{[333]} \\
{[456 *, 499 *]} \\
{[500 *, 501]} \\
{[502]} \\
{[503 *, 504 *, 505 *]}\end{array}$ \\
\hline $\mathrm{CO}$ & $\begin{array}{l}2.3 \mu \mathrm{m} \\
1.6 \mu \mathrm{m}\end{array}$ & \begin{tabular}{|l|} 
Exp \& Calc \\
Exp \\
Exp \\
\end{tabular} & $\begin{array}{l}{[238,332 *]} \\
{[477 *, 478 *]} \\
{[10],}\end{array}$ \\
\hline $\mathrm{CO}_{2}$ & $\begin{array}{l}\text { All in HITRAN } \\
3 \mu \mathrm{m} \\
1.6 \mu \mathrm{m} \\
2.05 \mu \mathrm{m} \\
2.3 \mu \mathrm{m} \\
4.3 \mu \mathrm{m}\end{array}$ & $\begin{array}{l}\text { Calc } \\
\text { Exp \& Calc } \\
\text { Exp } \\
\text { Calc } \\
\text { Exp } \\
\text { Exp \& Calc } \\
\text { Exp }\end{array}$ & $\begin{array}{l}{\left[309^{*}, 311^{*}, 314^{*}\right]} \\
{\left[313^{*}\right]} \\
{\left[304,305,308^{*}, 457^{*}, 460,506,507\right]} \\
{\left[304,305,307^{*}, 308^{*}\right]} \\
{\left[508,466^{*}\right]} \\
{[315]} \\
{\left[103^{*}\right]}\end{array}$ \\
\hline $\mathrm{N}_{2} \mathrm{O}$ & $4.5 \mu \mathrm{m}$ & Exp & [470] \\
\hline $\mathrm{HCN}$ & $14 \mu \mathrm{m}$ & Exp & [509] \\
\hline $\mathrm{HDO}$ & $\begin{array}{l}2.7 \mu \mathrm{m} \\
3.6 \mu \mathrm{m} \\
7.4 \mu \mathrm{m}\end{array}$ & $\begin{array}{l}\text { Exp } \\
\text { Exp } \\
\text { Exp }\end{array}$ & $\begin{array}{l}{\left[286^{*}\right]} \\
{\left[285^{*}\right]} \\
{\left[281^{*}\right]}\end{array}$ \\
\hline $\mathrm{H}_{2} \mathrm{O}$ & $\begin{array}{l}\text { Some doublets } \\
2.5-2.9 \mu \mathrm{m} \\
2.5-5.4 \mu \mathrm{m}\end{array}$ & $\begin{array}{l}\text { Exp } \\
\text { Exp } \\
\text { Exp }\end{array}$ & $\begin{array}{l}{\left[510^{*}, 511\right]} \\
{[282 *]} \\
{[440 *, 447]}\end{array}$ \\
\hline $\mathrm{NH}_{3}$ & $3 \mu \mathrm{m}$ & Calc & {$[231,299,300]$} \\
\hline $\mathrm{N}_{2}$ & Iso Raman & Calc & {$[228,229,237]$.} \\
\hline $\mathrm{O}_{2}$ & $\begin{array}{l}0.76 \mu \mathrm{m} \\
118 \mathrm{GHz}\end{array}$ & \begin{tabular}{|l} 
Calc \\
Exp \\
Exp \\
\end{tabular} & $\begin{array}{l}{\left[318^{*}, 306,245\right]} \\
{\left[100^{*}, 494,245,306\right]} \\
{\left[512^{*}, 488\right]}\end{array}$ \\
\hline $\mathrm{PH}_{3}$ & $4.5 \mu \mathrm{m}$ & Exp & [481] \\
\hline
\end{tabular}

Table 4: Bibliography providing data in order to describe line-mixing effects. The references marked with an asterisk (eg [254*]) are those in which LM parameters were investigated at various temperatures.

\subsection{Far wings and associated continua}

This section reviews the data available for the modeling of the continuum-like absorptions to which the far line wings make a significant contribution (the CIA continua due 
to the dipole induced during collisions are the subject of Sec. 4.4). It completes the information given in Tables IV.5 and IV.6, as well as in Appendix V.A of [1], by providing references of studies for $\mathrm{H}_{2} \mathrm{O}$ and $\mathrm{CO}_{2}$ (which are, to our knowledge, the only species for which new values have been made available in the last decade).

\subsubsection{The water-vapor continua}

More than one century after its discovery ( $c f$ [513,514] and the review in [357]), the strength and physical origin of the absorption continua of water vapor remain an open question. In particular, the question of the respective contributions of the monomer line wings, of the stable and metastable dimers and of collision-induced absorption is not yet settled, as discussed in appendix V.A of [1] and in [391,427,515-521]. On this issue, note that water dimers have been recently found to contribute to the continuum in the $14-35 \mathrm{~cm}^{-1} /$ wave number region [522]. Partly resolved absorption features due to water dimers have also been identified in room-temperature spectra in the millimeter-wave range [523], the water dimer relative abundance being on the order of $10^{-3}$ at room temperature and $1.7 \mathrm{kPa}$.

A better characterization of the amplitude and temperature-dependence of the continuum is needed because of its contribution to the radiative budget of the Earth and thus to climate change through feedback effects [524-528] as well as its influence on the estimated extensions of the habitable zone of stars [529-531] (see also Sec. 5.3). Before describing recent results on the water-vapor continua, recall that they are generally divided into the socalled self-continuum for pure $\mathrm{H}_{2} \mathrm{O}$ and the foreign/continuum when $\mathrm{H}_{2} \mathrm{O}$ is mixed with another gas (generally $\mathrm{N}_{2}$ or air).

\subsection{1a The self-continuum}

Table 5 presents a review of (recent) measurements of the self-continuum, which is believed to be mostly exhaustive above (and including) the $10 \mu \mathrm{m}$ window (readers are invited to consult $[357,370]$ for longer wavelengths). Recall that, because of the weakness of the broadband absorptions to be measured, high sensitivity methods and/or long paths must be used. We have thus separated, in Table 5, the studies using long (multipass) cells and covering large spectral intervals mostly with FTS instruments from laser-based methods probing narrow spectral intervals. The main recent FTS studies were carried out by the CAVIAR consortium [373,532] with a 512.7 m path length at $293 \mathrm{~K}$ in England, with a $612 \mathrm{~m}$ path length at room temperature in Russia (Tomsk) and with a $100 \mathrm{~m}$ path length $(T=311 \mathrm{~K}$ to $363 \mathrm{~K}$ ) in the USA (NIST). Concerning laser-based methods, they have been used in the water-vapor transparency windows because of their inherent higher sensitivity. They include calorimetric-interferometry and cavity-enhanced methods ( $c f$ Sec. 2) such as CRDS and OFCEAS.

\begin{tabular}{|c|c|c|c|c|}
\hline \multicolumn{5}{|c|}{ Long path cell methods } \\
\hline $\begin{array}{c}\text { Spectral range } \\
\left(\mathbf{c m}^{-1} \mathbf{)}\right.\end{array}$ & Technique & Path length $(\mathbf{m})$ & T (K) & Refs. \\
\hline $10-90$ & $\begin{array}{c}\text { Interferometric } \\
\text { spectrometer }\end{array}$ & 23.3 & $293-333$ & {$[533]$} \\
\hline $14-200$ & FTS & 151.75 & 296 & {$[522]$} \\
\hline $800-1250$ & FTS & $68-116$ & $326,339,352,363$ & {$[370]$} \\
\hline $800-1250$ & FTS & 100 & $311,326,352$ & {$[387]$} \\
\hline $2400-2640$ & Spectrograph & 707 & $296-428$ & {$[363]$} \\
\hline $1900-3500$ & FTS & 100 & $311-363$ & {$[384]$} \\
\hline $1900-2600$ & Spectrograph & 0.0529 & $500-875$ & {$[534]$} \\
\hline and 3900-4600 & & 128 and 512 & $296-351$ & {$[518,535]$} \\
\hline $1200-8000$ & FTS & 512.7 & 293 & {$[373]$} \\
\hline $2500-10000$ & FTS & 17.7 & $350-472$ & \\
\hline
\end{tabular}




\begin{tabular}{|c|c|c|c|c|}
\hline $1300-8000$ & FTS & 612 & 289.5 and 318 & {$[374]$} \\
\hline $2000-8000$ & FTS & 1065.5 & 287 & {$[375]$} \\
\hline \multicolumn{5}{|c|}{ Laser-based methods } \\
\hline $\begin{array}{l}\text { Measurement } \\
\text { points }\left(\mathrm{cm}^{-1}\right)\end{array}$ & Technique & Laser source & $\mathbf{T}(\mathbf{K})$ & Refs. \\
\hline $931,944,969$ & CRDS & $\mathrm{CO}_{2}$ & $294-297$ & [364] \\
\hline 944 & CRDS & $\mathrm{CO}_{2}$ & $270-315$ & [369] \\
\hline 2283 & OF-CEAS & QCL & $296,301,312$ & [380] \\
\hline 2491 & OF-CEAS & ICL & $297,312,325$ & [381] \\
\hline $4249-4257$ & CRDS & DFBL & 298 & [379] \\
\hline 4301 & OF-CEAS & DFBL & $297-323$ & [378] \\
\hline $4341-4367$ & CRDS & VECSEL & 297 & {$[380]$} \\
\hline $4427-4441$ & CRDS & DFBL & 296.2 & [381] \\
\hline $4516-4532$ & CRDS & DFBL & 297 & [380] \\
\hline 4605 & $\begin{array}{c}\text { Calorimetric } \\
\text { interferometry in air }\end{array}$ & $\begin{array}{c}\text { Raman-shifted } \\
\text { alexandrite }\end{array}$ & 298 & [382] \\
\hline 4723 & OF-CEAS & DFBL & $298-323$ & [378] \\
\hline $5875-6665$ (10 pts) & CRDS & DFBL & $302-340$ & {$[376,377]$} \\
\hline $6112-6165$ & $\begin{array}{c}\text { Calorimetric } \\
\text { interferometry in air }\end{array}$ & $\begin{array}{l}\text { Raman-shifted } \\
\text { alexandrite }\end{array}$ & & {$[382]$} \\
\hline $7500-7920$ & CRDS & DFBL & 297 & {$[380]$} \\
\hline $7920-8300$ & CRDS & ECDL & 297 & {$[380]$} \\
\hline 9466 & $\begin{array}{c}\text { Calorimetric } \\
\text { interferometry in } \mathrm{N}_{2}\end{array}$ & Nd:glass & 303 & {$[383]$} \\
\hline 11495 & $\begin{array}{c}\text { Pulsed CRDS } \\
\text { (upper limit in air) }\end{array}$ & Dye laser & 295 & {$[536]$} \\
\hline
\end{tabular}

Table 5: Summary of recent laboratory measurements of the self-continuum of water vapor. QCL:Quantum Cascade Laser, DFBL: Distributed Feed Back diode laser, VECSEL: Vertical External Cavity Surface Emitting Laser, ECDL: External Cavity Diode Laser, ICL: Interband Cascade Laser.

The measured values of the self-continuum absorption cross-sections between wave numbers of $1500 \mathrm{~cm}^{-1}$ and $10500 \mathrm{~cm}^{-1}$ are compared to the last version (V3.0) of the MT_CKD model in Fig. 15 (Sec. 3.5.4). A striking fact is the strong disagreement (up to two orders of magnitude near $6200 \mathrm{~cm}^{-1}$ ) between the FTS and laser-based methods in the transparency windows. Furthermore, the Tomsk and CAVIAR values are almost constant throughout the $1.6 \mu \mathrm{m}, 2.1 \mu \mathrm{m}$ and $4.0 \mu \mathrm{m}$ wavelength regions, with a value (about $4 \times 10^{-23}$ $\mathrm{cm}^{2}$ molec $^{-1} \mathrm{~atm}^{-1}$ ) largely above the MT_CKD predictions. Conversely, the OF-CEAS and CRDS measurements follow the general MT_CKD3.0 evolution with frequency, exhibiting very good agreement in the $1.6 \mu \mathrm{m}$ and $1.25 \mu \mathrm{m}$ windows but deviations by factors from 2 to 5 at the center of the $2.1 \mu \mathrm{m}$ and $4.0 \mu \mathrm{m}$ windows. Detailed discussion of this puzzling situation can be found in [380,537]. Note that during the derivation of the absorption cross section from OF-CEAS and CRDS spectra, the pressure-squared dependence of the selfcontinuum was systematically checked to insure the gas phase origin of the measured losses. Except for [384], all other FTS determinations near room temperature rely on spectra recorded for a single pressure.

The evolution of the self continuum near and below room temperature is obviously an important issue in studies of the atmosphere of the Earth, but it is difficult to measure because of the limitation imposed by the saturation pressure. Thus, the use of high temperatures makes the determinations easier. From this point of view, the most complete studies ( $c f$ Table 5) were performed at NIST between $311 \mathrm{~K}$ and $363 \mathrm{~K}$ [370,384,387] and by the CAVIAR consortium [373] in the $350 \mathrm{~K}$ to $472 \mathrm{~K}$ range. Interestingly, in the $4.0 \mu \mathrm{m}$ and $2.1 \mu \mathrm{m}$ wavelength windows, these high temperature measurements are consistent with room temperature results by OF-CEAS and CRDS [380,381], as shown in Fig. 18. In addition, as 
noted in [378,381], the measured values, including the earlier results of [363] (up to $428 \mathrm{~K}$ ) and of [534] (up to $875 \mathrm{~K}$ ), nicely follow a $\exp \left[-D_{0} /\left(k_{B} T\right)\right]$ law with a $D_{0}$ value close to the dissociation energy of the water dimer $\left(1100 \mathrm{~cm}^{-1}\right.$ [538]). However, as discussed in [378], the interpretation of this experimental result as an evidence of the dimer origin of the water continuum in the considered windows, seems hazardous in absence of a theoretical support. Indeed, theoretical calculations based on the far wings theory yield a $D_{0}$ value of $1800 \mathrm{~K}$ near $944 \mathrm{~cm}^{-1}$ [516], which is close to that of $1842 \mathrm{~K}$ retrieved from measurements between $270 \mathrm{~K}$ to $315 \mathrm{~K}$ [369] and to those of $1870 \mathrm{~K}, 1705 \mathrm{~K}$, and $1680 \mathrm{~K}$ obtained with three $\mathrm{CO}_{2}$-laser lines between $276 \mathrm{~K}$ and $366 \mathrm{~K}$ [368]. More generally, as mentioned at the very beginning of Sec. 4.3.1, the relative contribution of dimers and far wings of the monomer lines to the continuum is still an opened question. Finally note that Fig. 18 shows that the MT_CKD model predicts stronger temperature dependences, and that [373] provides other comparisons of this model with measurements at various temperatures.
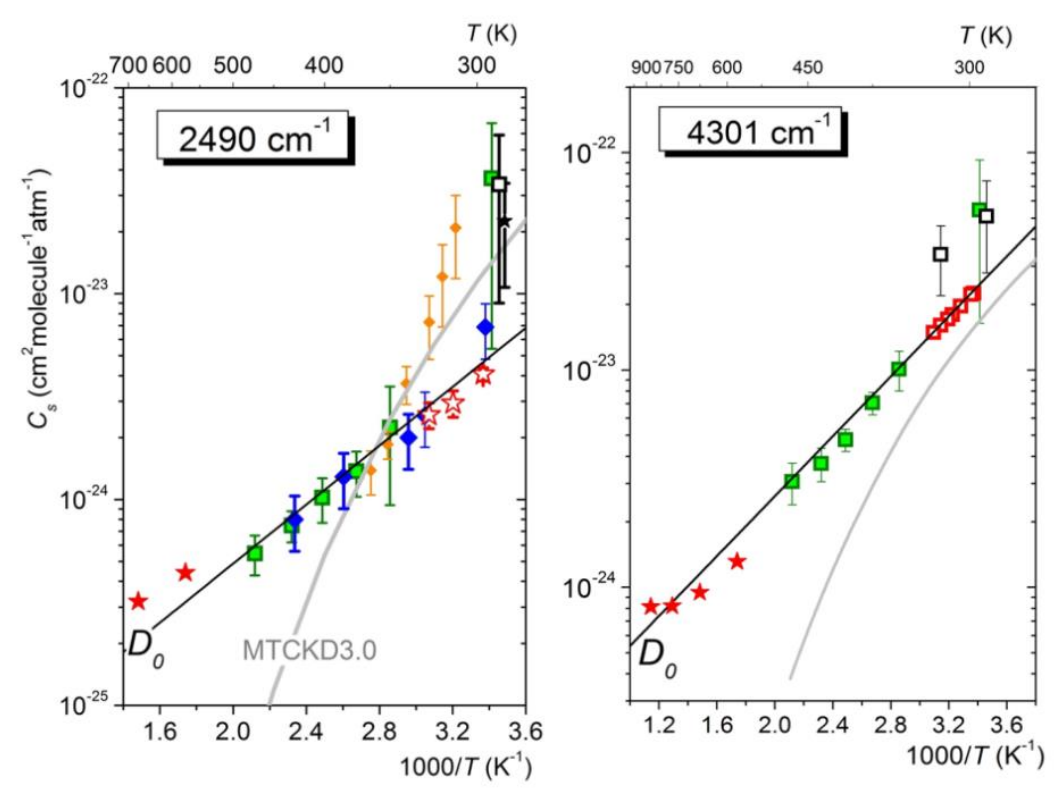

Fig. 18: Temperature dependence of the water vapor self-continuum cross-sections near 2490 and $4301 \mathrm{~cm}^{-1}$ obtained by FTS (green squares from [373]; orange diamonds from [384]; black star and squares from [374] and [375], respectively), using a grating spectrograph (blue diamonds from [385] and [362], red full stars from [534]) and OF-CEAS (open red stars from [379] and open red squares from [378]). The MT_CKD3.0 values (grey line) which are normalized to the number density at 1 atm and $296 \mathrm{~K}$ have been multiplied by the factor 296/T. The $D_{0}$ slope (black line) corresponds to an $\exp \left(-D_{0} / k_{\mathrm{B}} T\right), D_{0} \approx 1100 \mathrm{~cm}^{-1}$ being the dissociation energy of the water dimer molecule [538].

\subsection{1b The foreign-continua}

In our atmosphere, the air-continuum contribution to the absorption can be of the same order as the self-continuum contribution [379] and it represents a major source of uncertainty in Earth atmospheric radiative transfer models [386]. The analysis of atmospheric spectra thus provides valuable constraints to validate the continuum amplitude and its temperature dependence, in particular for the infrared and microwave regions. Nevertheless, the retrieval of water-vapor continuum absorption from atmospheric spectra is challenging due to the difficulty in discriminating it from aerosol scattering and absorption. Recent atmospheric validation in the infrared can be found in [539-543].

Table 6 regroups the few recent laboratory studies of the foreign continuum associated with mixtures of $\mathrm{H}_{2} \mathrm{O}$ with $\mathrm{N}_{2}$ (or air). The concordant experimental results obtained at NIST 
[387,388], by the CAVIAR consortium [386] and by CRDS in the $2.1 \mu \mathrm{m}$ window [379] have led to a significant increase of the MT_CKD foreign-continuum model in the transparency windows. Note that the foreign-continuum has been found to be mostly independent of temperature in the broad-band CAVIAR FTS measurements between $350 \mathrm{~K}$ and $430 \mathrm{~K}$ [386]. Similar results were reported in the infrared between $296 \mathrm{~K}$ and $363 \mathrm{~K}[387,388]$. Finally, the review of [544] concludes that the laboratory and field measurements of the continuum absorption coefficients at $183 \mathrm{GHz}$, do not exhibit a temperature dependence in the 260-360 $\mathrm{K}$ range.

\begin{tabular}{|c|c|c|c|c|c|}
\hline \multicolumn{7}{|c|}{ Long path cell methods } \\
\hline $\begin{array}{c}\text { Spectral range } \\
\left(\mathbf{c m}^{-1}\right)\end{array}$ & Technique & $\begin{array}{c}\text { Path length } \\
(\mathbf{m})\end{array}$ & Foreign gas & T (K) & Refs. \\
\hline $1000-2500$ & FTS & 100 & $\mathrm{~N}_{2}$ & 296,326 & {$[387]$} \\
\hline $2000-3230$ & FTS & 100 & $\mathrm{~N}_{2}$ & $\begin{array}{c}326,339,352, \\
363\end{array}$ & {$[388]$} \\
\hline $2000-10000$ & FTS & 17.7 & air & 350,372, & {$[386]$} \\
\hline \multicolumn{7}{|c|}{ Laser-based methods } & & \\
\hline $3.5-4.7$ & BWO & $0.35-0.7$ & $\mathrm{~N}_{2}$ & $261-328$ & {$[545]$} \\
\hline $4249-4257$ & CRDS & DFBL & air & 298 & {$[379]$} \\
\hline 10611 and & CRDS & ECDL & $\mathrm{N}_{2}$ & 278 and 296 & {$[546]$} \\
\hline 10685 & \multicolumn{2}{|c|}{$\begin{array}{c}\text { single-pulse } \\
\text { ruby laser }\end{array}$} & $\mathrm{N}_{2}$ & 295 & {$[547]$} \\
\hline $14397-14401$ & Photoacoustic & & & \\
\hline
\end{tabular}

Table 6: Recent laboratory studies of the foreign-continuum measurements of water vapor in $\mathrm{N}_{2}$ or air. BWO: Backward Wave Oscillator, QCL.Quantum Cascade Laser, DFBL:

Distributed Feed Back diode laser, VECSEL: Vertical External Cavity Surface Emitting Laser, ECDL: External Cavity Diode Laser.

\subsubsection{The $\mathrm{CO}_{2}$ far-wings continua}

As for the $\mathrm{H}_{2} \mathrm{O}$ continua, the far wings continua of $\mathrm{CO}_{2}$ play a major role in radiative transfer exchanges in planetary atmospheres (eg Sec. 5.3.3). In Table 7 are given several mostly recent studies in which the far-wings continua of $\mathrm{CO}_{2}$ have been determined from experiments. These involve different spectral regions and temperatures and were carried for pure $\mathrm{CO}_{2}$ and/or various mixtures including $\mathrm{N}_{2}$ and $\mathrm{H}_{2} \mathrm{O}$. Note that some of the references also provide empirical models.

\begin{tabular}{|c|c|c|c|c|c|}
\hline $\begin{array}{c}\text { Spectral } \\
\text { range }\left(\mathbf{c m}^{-1}\right)\end{array}$ & Technique & $\begin{array}{c}\text { P range } \\
(\mathbf{a t m})\end{array}$ & $\begin{array}{c}\boldsymbol{T} \text { range } \\
(\mathbf{K})\end{array}$ & $\begin{array}{c}\text { Path length } \\
(\mathbf{m})\end{array}$ & Refs. \\
\hline $800-30000$ & FTS & $0-200$ & $296-650$ & 0.02 & {$[548]$} \\
\hline $750-6000$ & FTS & $3-57$ & $298-550$ & $2.00-2.15$ & {$[311]$} \\
\hline $2380-2460$ & FTS & 1 & $230-296$ & 20 & {$[355]$} \\
\hline $2400-2600$ & FTS & $1-3$ & $295-339$ & 100 & {$[549]$} \\
\hline $2500-2600$ & FTS & $0-1$ & $325-366$ & 7.2 & {$[356]$} \\
\hline $3900-4600$ & FTS & $1-22$ & 297 & 120 & \multirow{2}{*}[550]{} \\
\hline $3900-4600$ & grating & $15-20$ & $290-293$ & $32-482$ & \\
\hline $3750-3900$ & FTS & $10-40$ & $299-301$ & 3 & \\
\hline $3750-3850$ & grating & $10-60$ & 292 & 0.07 & \\
\hline $4320-4380$ & CRDS & $0.3-1$ & 296 & & {$[551]$} \\
\hline $5850-5960$ & CRDS & $0-8.4$ & 296 & & {$[552]$} \\
\hline $5693-5795$ & CRDS & $0-10$ & 296 & & {$[553]$} \\
\hline $8449-8482$ & CRDS & $0-40$ & 293 & & {$[554]$} \\
\hline
\end{tabular}

Table 7: Recent laboratory studies of far wings continua of $\mathrm{CO}_{2}$. 


\subsection{Collision-induced absorption}

Several relatively recent references, in which CIA data obtained from measurements and/or calculations are presented, are regrouped in Table 8. In addition to those given below, other older studies can be found by consulting [389,432,433] and Sec. 3.6.

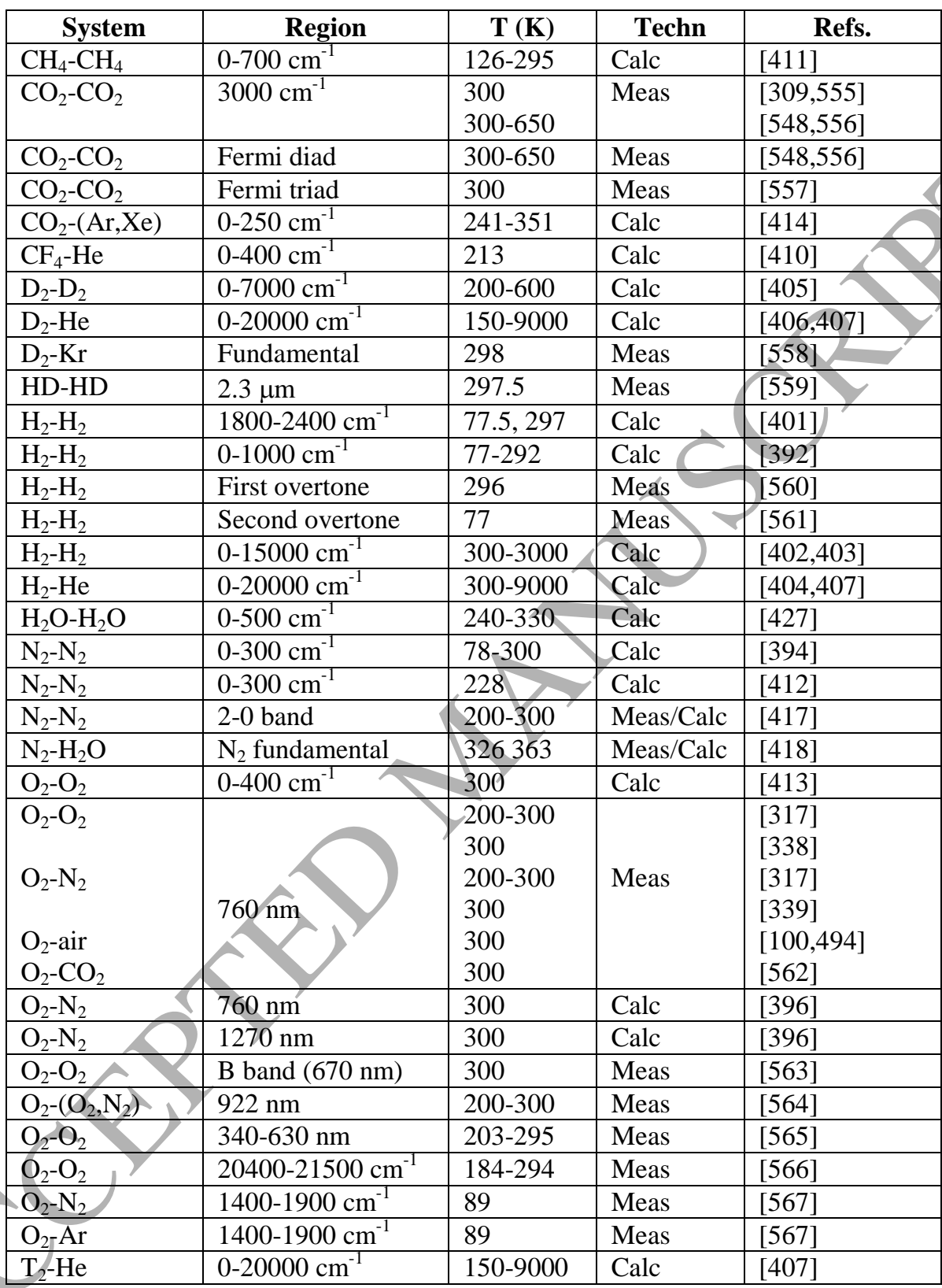

Table 8: Recent bibliography providing data on collision-induced absorption.

\subsection{Spectroscopic databases}

Since their creation almost fifty years ago, the (most) widely used spectroscopic databases HITRAN [430] and GEISA [431] have evolved constantly. However, until relatively recently, the provided line-shape information was quite limited and suitable only for calculations using the VP. As discussed below, significant advances have been made in the last decade to provide data for calculations under more general conditions.

\subsubsection{The HITRAN database}


For many decades, the HITRAN spectroscopic database [430] included only the air- and self-broadening coefficients at $296 \mathrm{~K}$. The temperature dependences of air-broadened halfwidths and the air pressure-induced shifts (at $296 \mathrm{~K}$ ) were added later. It was not until the 2012 edition [429] that some non-Voigt profile parameters were tabulated (SDVP parameters for the first overtone of $\mathrm{CO}$ and $\mathrm{GP}$ parameters for $\mathrm{HF}, \mathrm{HCl}$ and the $\mathrm{O}_{2}$ A-band). A breakthrough in the ability to seamlessly provide non-Voigt line-shape parameters, as well as LM (so far in the first-order parameterization [344]) and pressure-broadening and -shifting coefficients by planetary gases, came with changing the database from static ASCII files to a relational database [568]. The structure of interconnected tables (corresponding to every parameter and set of quantum numbers) enables the introduction of unlimited numbers of parameters and to query these quantities in a user-defined fashion and order. In order to ease this querying process, an online interface, called HITRANonline [439], was recently developed [569].

Since the HTP ( $c f$ Sec. 3.2.4a) was recommended [179] as a reference line-shape that is more and more frequently used in fits of measured spectra, an effort is underway to store its parameters (and thus also those of more simple profiles which are particular cases of the HTP) in HITRAN. A detailed description of how this is done is given in [153] where the $\mathrm{H}_{2}$ molecule was taken as a test case. Thanks to numerous measurements of the electric quadrupole lines of pure hydrogen, a complete dataset was obtained for every transition. Not only were all the HTP parameters accommodated but they were divided into different temperature domains. It is important to note that although the HTP can be reduced to several other models [169], it cannot replace all the profiles (eg GP) that already exist in the database. In addition, because every line in HITRAN retains a Lorentzian pressure-broadening coefficient which is traditionally determined and used with the VP, these parameters remain available. This result is important to remember since the half-widths values generally differ when extracted from measurements by fits with different line profiles (see below). Indeed, because it will take a long time before all radiative transfer codes are upgraded to use nonVoigt profiles, there is a danger of errors being introduced by applying the widths determined with a sophisticated line shape to the VP.

The top panel of Fig. 19 exemplifies the output format created to retrieve air-broadened SDVP parameters together with first-order LM coefficients (along with the line positions, and "usual" Lorentz-broadening coefficient derived with the VP). Here a tab-delimited output was chosen (for better visualization) and the available possibility to display the uncertainties was not used. The bottom panel of Fig. 19 shows the corresponding output for the first overtone of ${ }^{12} \mathrm{C}^{16} \oslash[477,478]$. One can see that the air-broadening coefficients (at $296 \mathrm{~K}$ ) for the $\operatorname{VP}\left(\gamma_{\mathrm{V}}\right.$ air $)$ are available for every line, unlike the SDVP parameters $\left(\gamma_{\mathrm{SDV}}^{0}\right.$ air and $\gamma_{\mathrm{SDV}}^{2}$ air $)$. It is also clear that the values $\left(\gamma_{\mathrm{V}}\right.$ air and $\gamma_{\mathrm{SDV}}^{0}$ air $)$ determined with different line-shapes (here VP and SDVP) are not identical. 

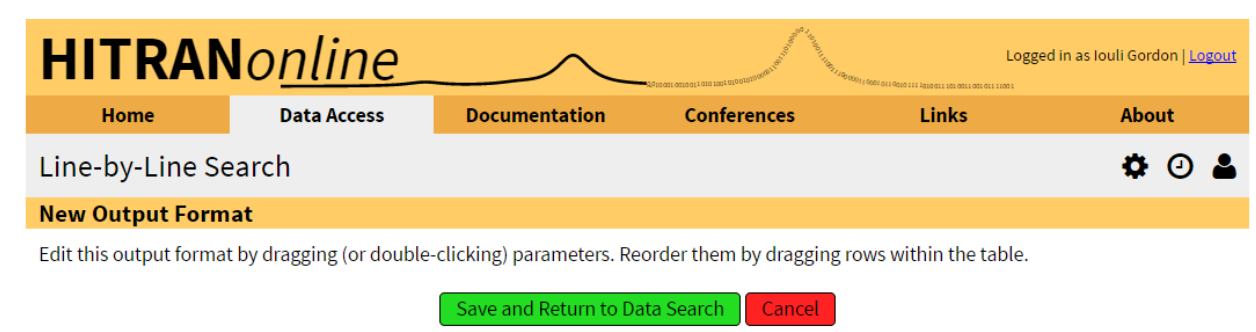

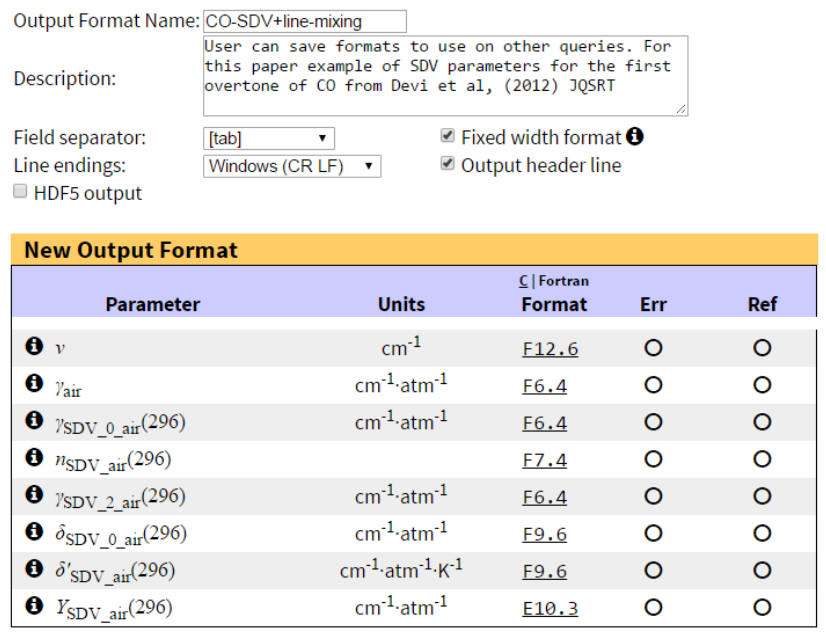

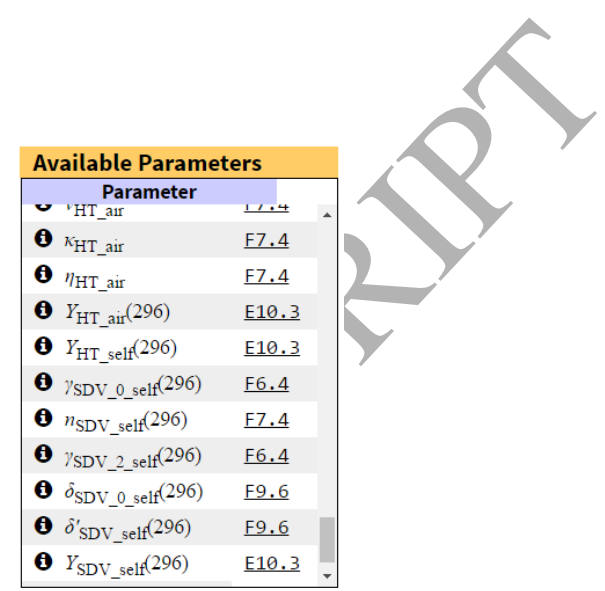

\begin{tabular}{|c|c|c|c|c|c|c|c|c|c|}
\hline$\sigma$ & $\gamma_{\mathrm{v}}$ air & $\gamma_{\mathrm{SDV}}^{0}$ air & $n_{\mathrm{SDV}}$ air & $\gamma_{\mathrm{SDV}}^{2}$ air & $\delta_{\mathrm{SDV}}^{0}$ air & $\delta_{\mathrm{SDV}}^{\prime}$ air & $Y_{\mathrm{SDV}}$ air & ref $\gamma_{\mathrm{v}}$ air & $\operatorname{ref} \gamma_{\mathrm{SDV}}^{0}$ air \\
\hline 4202.550466 & 0.0542 & \#\#\#\#\# & \#\#\#\#\#\# & \#\#\#\#\# & \#\#\#\#\#\#\#\# & \#\#\#\#\#\#\#\# & \#\#\#\#\#\#\#\# & 723 & 0 \\
\hline 4203.357916 & 0.0805 & \#\#\#\#\# & \#\#\#\#\#\# & \#\#\#\#\# & \#\#\#\#\#\#\#\# & \#\#\#\#\#\#\#\# & \#\#\#\#\#\#\#\# & 723 & 0 \\
\hline 4204.669501 & 0.0559 & 0.0551 & 0.7610 & 0.0036 & -0.004730 & 0.000025 & $1.400 \mathrm{e}-03$ & 723 & 721 \\
\hline 4205.187898 & 0.0535 & \#\#\#\#\#\# & \#\#\#\#\#\# & \#\#\#\#\#\# & \#\#\#\#\#\#\#\# & \#\#\#\#\#\#\#\# & \#\#\#\#\#\#\#\#\# & 723 & 0 \\
\hline 4207.752983 & 0.0526 & \#\#\#\#\#\# & \#\#\#\#\#\# & \#\#\#\#\#\# & \#\#\#\#\#\#\#\# & \#\#\#\#\#\#\#\# & \#\#\#\#\#\#\#\#\# & 723 & 0 \\
\hline 4209.343184 & 0.0566 & 0.0558 & 0.7702 & 0.0037 & -0.004700 & 0.000025 & $1.400 \mathrm{e}-03$ & 723 & 721 \\
\hline 4210.245573 & 0.0518 & \#\#\#\#\# & \#\#\#\#\#\# & \#\#\#\#\# & \#\#\#\#\#\#\#\# & \#\#\#\#\#\#\#\# & \#\#\#\#\#\#\#\# & 723 & 0 \\
\hline 4210.907967 & 0.0804 & \#\#\#\#\# & \#\#\#\#\#\# & \#\#\#\#\# & \#\#\#\#\#\#\#\# & \#\#\#\#\#\#\#\# & \#\#\#\#\#\#\#\# & 723 & 0 \\
\hline 4212.665523 & 0.0510 & \#\#\#\#\# & \#\#\#\#\#\# & \#\#\#\#\# & \#\#\#\#\#\#\# & \#\#\#\#\#\#\#\# & \#\#\#\#\#\#\#\# & 723 & 0 \\
\hline 4213.948622 & 0.0573 & 0.0564 & 0.7796 & 0.0040 & -0.004620 & 0.000025 & $1.600 \mathrm{e}-03$ & 723 & 721 \\
\hline 4214.577840 & 0.0753 & \#\#\#\#\# & \#\#\#\#\#\# & \#\#\#\#\# & \#\#\#\#\#\#\#\# & \#\#\#\#\#\#\#\#\# & \#\#\#\#\#\#\#\#\# & 723 & 0 \\
\hline
\end{tabular}

Fig. 19. Example of creating custom output formats and corresponding outputs.

In order to aid users in applications of non-Voigt line shapes, special libraries for the HITRAN Application Programming Interface (HAPI) [570] were created. As explained in [570], HAPI can download data from HITRANonline [439] with the option of retrieving all line-shape parameters, specific ones, or bundles of parameters ( $\mathrm{g}$ all those corresponding to a specific line shape). One can also employ the provided functions to calculate absorption cross-sections using any line-profile data available in the database. It is also possible to use a priority scheme whereby the program will first check whether the HTP parameters are available, then for the SDVP, and finally for the VP parameters. In this manner, one can run a calculation in which the best available profile is selected for every transition.

As detailed in [430], the HITRAN2016 edition provides parameters of the HTP for some transitions of $\mathrm{H}_{2} \mathrm{O}, \mathrm{N}_{2} \mathrm{O}, \mathrm{O}_{2}$ (B-band) and $\mathrm{H}_{2}$, of the SDVP for $\mathrm{CO}$ and $\mathrm{O}_{2}$ (A-band), and of the $\mathrm{GP}$ for $\mathrm{HF}, \mathrm{HCl}$ and $\mathrm{O}_{2}$ (A-band). First-order line-mixing data at different temperatures have also been added in the database for some transitions of several molecules including $\mathrm{H}_{2} \mathrm{O}, \mathrm{N}_{2} \mathrm{O}, \mathrm{CO}$ and $\mathrm{O}_{2}$. The full line-mixing (relaxation matrix approach) is provided for $\mathrm{CO}_{2}$ through auxiliary program from [314] adjusted to work with the HITRAN2016 data. Another important recent development is that VP parameters caused by collisions with $\mathrm{CO}_{2}, \mathrm{H}_{2}$ and $\mathrm{He}$ were introduced for $\mathrm{CO}, \mathrm{SO}_{2}, \mathrm{NH}_{3}, \mathrm{HF}, \mathrm{HCl}, \mathrm{OCS}$ and $\mathrm{C}_{2} \mathrm{H}_{2}$ 
[571]. This was done for applications to planetary atmospheres and these parameters can be easily retrieved from HITRANonline [439] and through HAPI. Note that HAPI also enables calculations of the absorption cross-sections for mixtures of perturbing gases. Finally recall that, in 2012, a new section providing CIA data was added to the HITRAN database [432], now being updated [433].

\subsubsection{The GEISA database and 4A/OP code}

As with the HITRAN database described above, GEISA [431] has included for many decades air- and self-broadening coefficients at $296 \mathrm{~K}$, while the air-pressure shift and the temperature dependences of air-broadened half-widths were introduced later. Since the 2011 edition, the statistical uncertainty (precision) of these parameters has been added.Contrary to HITRAN, GEISA still only provides parameters for the VP, even in its 2015 edition [431]. This is because the choice was made to let radiative transfer codes account for other line profiles, LM and CIA. This is the case, for example, of the spectrum calculation code that builds the look-up tables feeding the 4A/OP radiative-transfer algorithm [572-574] which is developed by the research group in charge of GEISA. Using the link between GEISA and 4A/OP, a quasi-automatic chain has been developed for the validation not only of the radiative-transfer calculation but also of the spectroscopic parameters including those for the line shape [575]. This enables to carry comparisons between computed and measured spectra and to eventually point out discrepancies due to improper spectroscopic data. When it is the case, the values of the relevant parameters are, when possible, improved and changed in the database.

The current GEISA and 4A/OP package includes numerous spectrum-computation possibilities going beyond the VP. The latter have been selected for their importance in the treatment of the observations of several satellite-based instruments in which the group in charge of these tools is/was deeply involved (eg IÁSI [576], MicroCarb [577] and MERLIN [578]). They are the following: (i) LM and SDVP for $\mathrm{CO}_{2}$ lines (including the effects of collisions with dry air and water vapor), as described in [314] but starting from the GEISA line list. (ii) LM in the $\mathrm{O}_{2} \mathrm{~A}$ band following [318]. (iii) LM and HTP for the $\mathrm{R}(6)$ manifold of the $2 v_{3}$ band of $\mathrm{CH}_{4}$ from [9]. (iv) CIA by $\mathrm{O}_{2}$ in the $0.76 \mu \mathrm{m}$ [318], $1.27 \mu \mathrm{m}$ [579] and $6.3 \mu \mathrm{m}$ [580] regions. (v) CIA in the fundamental band of $\mathrm{N}_{2}$ built from an improved version [581] of the tools proposed in [582]. Note that the currently implemented LM models for $\mathrm{CO}_{2}$ and $\mathrm{O}_{2}$ have been recently completed by the possibility to use parameters from other sources $[100,466,506,507]$ for some bands. For $\mathrm{CH}_{4}$, the implementation of LM for the $v_{4}, v_{3}$ and $2 v_{3}$ bands is currently under progress, based on updates of [333,583]. Finally, for water vapor, the $\mathrm{VP}$ is used up to $300 \mathrm{~cm}^{-1}$ away in the line wings. As a consequence, the corresponding 4A/OP water vapor continuum is not the "MT_CKD" (which uses a $25 \mathrm{~cm}^{-1}$ cut-off distance and is described in Sec. 3.5.4) but something that has been refitted based on the validation tools described in [575].

\section{Consequences for applications}

The fact that the information contained in molecular spectra can be used to probe gases has been used for decades. The associated remote-sensing studies investigate (Sec. 5.1) a large variety of media, including planetary atmospheres and combustion environments, involving very diverse pressure and temperature conditions. In addition to these applications, advances in both experimental techniques and line-shape models have opened the possibility of using spectroscopy for some metrology purposes which require extreme accuracies (Sec. 5.2), such as the determination of the Boltzmann constant from precise measurements of the Doppler width. The success of such studies, which often requires extreme precision and 
accuracy, relies on the quality of the description of the spectral shapes and constitutes a great challenge to this research field. Finally, gases participate in radiative heat exchange in various systems at different spatial scales (Sec. 5.3). It is the case in combustion engines, for instance, where gaseous emission and absorption influence the hydrodynamics and the energy fluxes to bounding solid surfaces. At much larger spatial scales, they contribute to the greenhouse effect, for instance, and the accuracy of models involving absorption by atmospheric gases affects the reliability of climate predictions. These examples show that a description of the effects of pressure, temperature and composition on molecular-gas spectra is required in a variety of other research fields tackling diverse issues. The latter being too numerous to be all considered here, we have limited ourselves to some illustrative examples (as in Chapt. VII of [1]) highlighting the practical advances achieved because of recent spectral-shape studies.

\subsection{Remote sensing}

\subsubsection{Probing atmospheres}

\subsection{1a The Earth}

A striking recent evolution of some Earth-observation satellite-based experiments is the considerably increased retrieval accuracy that is required for their success. This is the case, for instance, of current or upcoming missions for the study of greenhouse gases [577,578,584587]. Indeed, reliable and scientifically worthwhile inversions of sources and sinks of $\mathrm{CO}_{2}$ and $\mathrm{CH}_{4}$ determined from atmospheric column amounts require spatially resolved measurement biases less than $0.1 \%$ and $0.2 \%$, respectively. Enabled by highly precise forward models for $\mathrm{O}_{2}$ and $\mathrm{CO}_{2}$ absorption, the OCO-2 mission recently reported an unprecedented precision in the measured $\mathrm{CO}_{2} \mathrm{VMR}$ of $\sim 0.1 \%$, sufficient to reveal previously unobserved regional and temporal trends in the global carbon cycle [588]. We now discuss how recent progress in the spectral-shape description (including isolated lines, LM, far wings and associated continua as well as CIA) has improved various optical soundings of the Earth atmosphere. Some general comments on the spectral-shape issue in atmospheric retrievals are made at the end of this section.

- Isolated line shapes: While it is obvious that errors in the input pressure-broadening and -shifting coefficients can generate large atmospheric-spectra fit residuals and subsequent errors in the targeted retrieved quantity, there are relatively few demonstrations of the need to go beyond the VP. The reasons for this are likely that non-Voigt effects (Sec. 3.2) are generally small and/or masked by the noise and limited spectral resolution of many observations and/or smaller than residuals caused by the incorrect accounting of other effects (such as line-mixing). Furthermore, in some retrieval methods, the molecular amount-ofsubstance information depends mainly on the line area, so that refined line-shape effects are generally small. This was first shown by in situ measurements of atmospheric $\mathrm{H}_{2} \mathrm{O}$ using a balloon-borne diode-laser spectrometer [589]. A RP (hard collisions), which accounts for Dicke narrowing, gave significantly better fits to the spectra, but the change in the retrieved $\mathrm{H}_{2} \mathrm{O}$ VMR was only $0.15 \%$. More recently, the effect of the SDVP on water vapor profiles retrieved from ground-based FTIR solar-absorption spectra was investigated [590]. A better agreement with in situ sonde profiles was obtained than when using a purely VP. The effects of the speed-dependence of the pressure broadening was also investigated (theoretically) in the case of $\mathrm{O}_{3}$ retrievals [591] from limb occultation, limb emission, and ground-based observations. The conclusion of this study is that the effect is minor $(<1 \%)$ in all three cases. Following [592], who pointed out the effects of Dicke narrowing in retrievals of $\mathrm{HCl}$ and $\mathrm{HF}$ from ground-based solar absorption FTIR spectra, a similar study was made [593] for HF only. It showed that use of the GP, rather than the VP, results in a substantial improvement in the HF fitting residuals, with smoother retrieved HF VMR profiles between $25-40 \mathrm{~km}$, but differences in the total column abundances of only $\sim 1 \%$. Finally, TCCON (Total Carbon 
Column Observatory Network, see [594]) retrievals of atmospheric $\mathrm{CO}_{2} \mathrm{VMR}$, which rely on column-integrated observations of the $\mathrm{O}_{2}{ }^{1} \Delta$-band at $1.27 \mu \mathrm{m}$, showed reduced airmass dependence and bias with a forward model for $\mathrm{O}_{2}$ absorption that used the SDVP instead of the VP [595] (the airmass being defined as the ratio of the total number of molecules within the considered line of sight to that for a vertical column). Empirical corrections for these two effects were reduced by factors of 6 and 2.6, respectively.

- Line-mixing: The fact that taking LM effects into account may improve calculations and analyses of atmospheric spectra has been discussed in Sec. VII.4 of [1] and references therein. This has since been confirmed in the spectral regions used by several satellite missions for the retrieval of column amounts of $\mathrm{CO}_{2}$ and $\mathrm{CH}_{4}$ and the determination of surface pressure from $\mathrm{O}_{2}$ absorption, as discussed below.

For the P- and R-branch manifolds of the $2 v_{3}$ band of $\mathrm{CH}_{4}$ used for satellite monitoring [578,584], LM effects were investigated using laboratory spectra in [333]. This study also showed that taking LM into account leads to improved fits of TCCON atmospheric transmission spectra and to a reduced airmass dependence of the retrieved column amounts. Earlier, large fitting residuals, and retrieved $\mathrm{CH}_{4}$ column abundances at noon that are $6 \%$ larger than those at high airmasses (in the early morning and late afternoon), were obtained [596] and explained by the use of wrong line widths. Fits of laboratory spectra in the $2 v_{3}$ band using the VP were then performed [597], leading to "effective" pressure-broadening and shifting coefficients. Although this is a crude way [333] to take LM into account, it is more convenient and can still lead to accurate column abundances, at least for pressures of $101 \mathrm{kPa}$ (1 atm) and below [333]. For the SCIAMACHY satellite instrument, the changes in the widths and shifts implied a latitudinal and seasonally varying bias of $1 \%$ in the total $\mathrm{CH}_{4}$ column when using the HITRAN 2004 line list [597]. Very recent results on $\mathrm{CH}_{4}$ retrievals using the $2 v_{3}$ band can be found in [598] where LM and SDVP derived from laboratory spectra are used, showing that the former is the major player in the observed improvements. Note that atmospheric $\mathrm{CH}_{4}$ measurements were also made using a balloon-borne diode-laser spectrometer centered on the R(6) manifold of the $v_{3}$ band [500]. It was shown that use of LM together with a RP gave improved fits to the atmospheric spectra and caused $3 \%$ to $7 \%$ decreases in the retrieved $\mathrm{CH}_{4}$ at $8 \mathrm{~km}$ to $18 \mathrm{~km}$ altitude (in better agreement with the in situ cryo-sampler on board the same gondola), relative to a VP without LM (but, contrary to what was done in [596], here using the "true" broadening parameters). The relative contributions of the RP versus LM to the $\mathrm{CH}_{4}$ changes were not discussed. For completeness, recall a somehow similar study for the $\mathrm{P}(9)$ manifold of the $v_{3}$ band [599].

For $\mathrm{CO}_{2}$, the importance of $\mathrm{LM}$ in the bands commonly used for observations from space [577,584-586] was first demonstrated in [600]. This study showed that including LM in the forward model leads to a significant reduction of the residuals in the $2.1 \mu \mathrm{m}$ band (from up to $2 \%$ down to typically $0.5 \%$ ). It also reduces the dependence of the retrieved $\mathrm{CO}_{2}$ on the airmass and improves the consistency between values obtained independently from spectra in the $1.6 \mu \mathrm{m}$ and $2.1 \mu \mathrm{m}$ bands. That study, in which calculated LM coefficients were used, was subsequently improved by taking the speed dependence into account and using LM data obtained from fitting laboratory spectra [436,601]. [436] confirmed that, by using LM and a SDVP, the $\mathrm{CO}_{2}$ spectral fitting residuals in ground-based TCCON spectra are improved and the airmass dependences of the retrieved $\mathrm{CO}_{2}$ total columns are substantially reduced (from a few \% down to a few $0.1 \%$ ). As for the case of $\mathrm{CH}_{4}$ discussed above and in [598], $\mathrm{LM}$ is the main factor in these improvements. Indirect consequences of $\mathrm{LM}$ in $\mathrm{CO}_{2}$ have also been pointed out in the ground-based observations of $\mathrm{CCl}_{4}$. Indeed, the total columns of this species retrieved from its $v_{3}$ band signature at $794 \mathrm{~cm}^{-1}$ depend on the quality of the modeling of the nearby $\left(791 \mathrm{~cm}^{-1}\right) \mathrm{CO}_{2}$ Q-branch and are on average $15 \%$ higher when the influence of $\mathrm{LM}$ on the $\mathrm{CO}_{2}$ absorption is disregarded in the forward model [602]. Finally, the importance 
of LM for the determination of $\mathrm{CO}_{2}$ amounts using the LIDAR instrument CO2LAS is discussed in [508].

For $\mathrm{O}_{2}$, the importance of LM (and CIA, see below) in the A band used for the determination of surface pressure from space [577,584-586], was first demonstrated in [318] using TCCON spectra. As for $\mathrm{CH}_{4}$ and $\mathrm{CO}_{2}$ (see above), both the fitting residuals and the airmass dependence of retrieved $\mathrm{O}_{2}$ were reduced. This study, in which calculated LM coefficients were used, was subsequently improved by taking the speed dependence into account and using LM and CIA data obtained from theory and by fitting laboratory (CRDS and FTS) as well as atmospheric (TCCON) spectra [100].

Finally, the effects of $\mathrm{LM}$ on atmospheric $\mathrm{O}_{3}$ retrievals were investigated [603] through simulations done for solar transmission and nadir emission. The results show that LM effects lead to systematic signatures in the fit residuals of typically $\sim 2 \%$ whose magnitude depend on the spectral resolution of the observation. The consequences for the retrieved total column ozone amount are quite modest, with errors of $2 \%$ in the worst situation, a result explained by the fact that $\mathrm{O}_{3}$ is primarily a stratospheric gas.

- Far wings and associated continua: We have found no paper discussing the influence of far wings and associated continua on retrievals from atmospheric absorption spectra. The reason is likely that, in this case, the former only appear as a constant (that is adjusted) multiplying the transmission over the relatively narrow spectral intervals selected for the retrieval. This is not the case in atmospheric emission in which a precise modeling of the broad-band contribution to the absorption/emission in the yarious atmospheric layers is required even though only a narrow interval is used for analysis. This issue, which is relevant for the $\mathrm{H}_{2} \mathrm{O}$ and $\mathrm{CO}_{2}$ continua only, is discussed from several remote sensing points of view in [544,604-608] and references therein.

- Collision-induced absorption: Concerning the importance of the correct modeling of CIA in remote sensing studies of the Earth atmosphere, there have been relatively few studies in the last decade. However, the need to include CIA in the $\mathrm{O}_{2} \mathrm{~A}$ band for accurate retrievals of surface pressure from space was demonstrated in $[318,609]$. Recently, a model for the CIA in the weak 2-0 band of $\mathrm{N}_{2}$ was proposed and validated using ground-based atmospheric transmission measurements [417]. It was there shown that disregarding the CIA leads to errors in the retrieved amounts of several trace gases. A quite original use of the $\mathrm{N}_{2}$ CIA in the fundamental band was proposed in [610] for the determination of the pointing in limbviewing measurements. This enables [610,611], once the pointing is known, an independent determination of the $\mathrm{CO}_{2}$ vertical profile.

- Some general comments on the spectral-shape issue in atmospheric retrievals: The ACOS OCO-2 retrieval algorithm [601,612,613] for the treatment of OCO-2 [585] spectra uses a full-physics forward model that includes LM and a SDVP. This is despite the fact that the OCO-2 resolution $\left(0.25 \mathrm{~cm}^{-1}\right.$ at $6200 \mathrm{~cm}^{-1} ; 0.7 \mathrm{~cm}^{-1}$ at $\left.13100 \mathrm{~cm}^{-1}\right)$ is insufficient to see any difference in the residuals obtained with the VP and SDVP. However, because many of the observed $\mathrm{CO}_{2}$ and $\mathrm{O}_{2}$ lines are saturated, the non-Voigt line shape does change the equivalent widths of the lines, which is reflected in the retrieved column amounts. In contrast, the official TCCON retrievals [594] are performed with a VP and no LM, despite having a spectral resolution of $0.02 \mathrm{~cm}^{-1}$ and a SNR of 600 sufficient to discern the subsequent degradation in the residuals [436,598]. The resulting airmass dependence of the column amounts is corrected post-retrieval by exploiting the fact that the retrieval error in the TCCON windows caused by the neglect of LM (along with other factors that also cause airmassdependent artifacts) is linear for small airmasses and consistent between summer and winter, as illustrated in Fig. 20. 

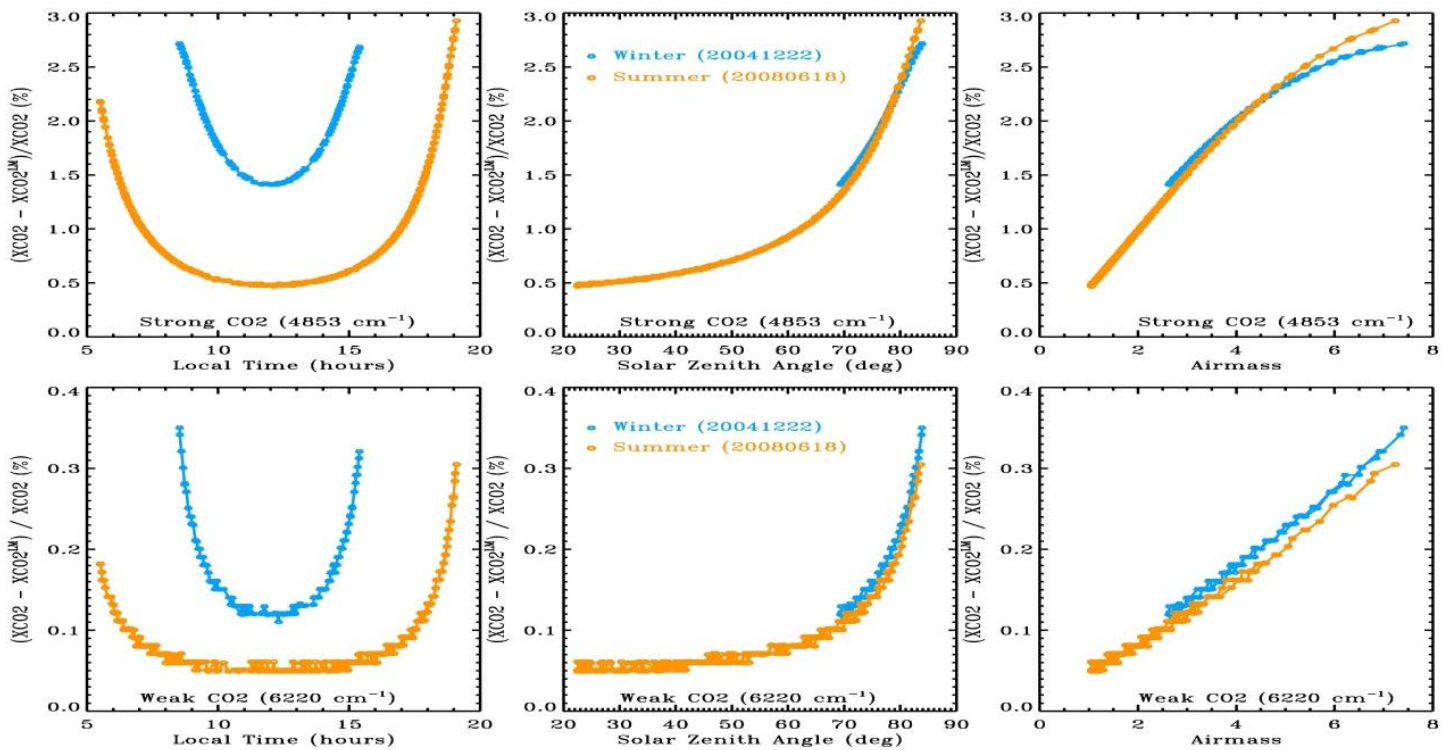

Fig. 20: Relative differences between the $\mathrm{CO}_{2}$ mole fractions retrieved from TCCON obtained when line-mixing is taken into account $\left(\mathrm{XCO}_{2}{ }^{\mathrm{LM}}\right)$ and disregarded (XCO2) in the forward model for the strong $\mathrm{CO}_{2}$ band centered at $4853 \mathrm{~cm}^{-1}$ (top panels) and the weak $\mathrm{CO}_{2}$ band centered at $6220 \mathrm{~cm}^{-1}$ (lower panels). The results for summer (orange) and winter (blue) are plotted versus local time (left), solar zenith angle (middle), and airmass (ie the ratio of the total number of molecules within the considered line of sight to that for a vertical column, right).

Recurrent themes in the above literature survey is that line-width errors and neglect of LM lead to significant residuals in the spectral fits and errors in retrievals gases $\left(e g \mathrm{CO}_{2}, \mathrm{CH}_{4}\right)$ requiring high precision. In retrievals that try to exploit the line shape to derive a vertical VMR profile (eg $\mathrm{HCl}, \mathrm{HF}, \mathrm{H}_{2} \mathrm{O}$ ), the assumed line shape is also important, but less so if the total vertical column suffices. The effeets of speed dependence are more subtle than those of width errors or LM and harder to discern among the other confounding factors that can affect the shapes of atmospheric open-path absorption lines (eg, the assumed T/P/VMR profiles, the field of view, the instrument line shape, zero-level offsets). Their effect on the retrieved gas amounts cannot be easily generalized since it depends on the measurement technique and analysis method. Consider a simple case of a DIAL LIDAR measuring at narrow and discrete wavelengths on and off the absorption line of interest. The inferred gas amount will obviously be sensitive to the values of the assumed line shape at the spectral points sounded. Even when measurements cover the entire line profile ( $e g$ FTS spectra, newer LIDARs (eg [614]) they can be analyzed in different ways. With analyses that use only the areas of the absorption lines, the inferred gas amounts are less sensitive to the line-shape details, especially when the lines are unsaturated. On the other hand, techniques that try to retrieve a vertical VMR profile from the shape of the spectral lines tend to be much more sensitive to the assumed line profiles.

Let us finally recall that, since the atmosphere on Earth involves temperatures as low as about $190 \mathrm{~K}$, forward models should include a precise description of the effects of temperature on all spectral features used for retrievals. However, testing the needed information brought by theory and laboratory experiments (Sec. 4) by comparisons between computed and measured atmospheric spectra is not so easy. Indeed, except when collected in situ, the latter involve contributions from numerous atmospheric layers that are at different temperatures. This problem can be partly solved by using limb recordings for various viewing angles, since the tangent layer makes a large contribution. But this approach has its limits since, as the temperature goes down with increasing tangent height, so does the pressure and the resulting collisional effects. For ground-based measurements, in which the lower layers 
generally make the largest contributions to the total absorption, a solution is to use spectra measured on warm and cold days, as in the example of Fig. 20.

\subsection{1b Other planets}

Spectroscopic databases such as HITRAN [430] or GEISA [431] still provide, for many absorbing molecules and lines, only the self- and air-broadened half widths (but an effort is underway [571] to complete HITRAN with parameters for other collision partners, $c f$ Sec. 4.5). However, these parameters are rarely relevant to planetary atmospheres that involve different major gases (eg $\mathrm{CO}_{2}$ for Venus and Mars, $\mathrm{H}_{2}$ for gas giants) and/or temperature and pressure conditions that are more extreme than on Earth. Nevertheless, there has been much experimental and theoretical effort in the past decade to derive parameters appropriate to various planetary environments, resulting in significant and accessible datasets. This is particularly the case for the pressure-broadening and -shifting coefficients induced by collisions with $\mathrm{CO}_{2}$ (relevant for Mars and Venus) of various molecules including (not exhaustively) $\mathrm{CO}$ [615], $\mathrm{H}_{2} \mathrm{O}$ [268], $\mathrm{HDO}[278,616], \mathrm{HCl}$ and $\mathrm{DCl}$ [617] (see also Tables 2 and 3). These data are now routinely used in the analysis of visible and infrared observations to derive the VMRs of these species and/or isotopic ratios in the atmospheres of Mars (eg [618-620]) and Venus (eg [621-623]). In particular, [624] discusses the sensitivity of the 1.38$\mu \mathrm{m}$ absorption band of water (and the subsequent retrieved water vapor content in the atmosphere of Mars) to spectroscopic databases and $\mathrm{CO}_{2}$-broadening coefficients.

Another hot topic is the detection (and retrieval) of $\mathrm{CH}_{4}$ in the atmosphere of Mars, which has consequences for its current biological or geological activity. This molecule might be present at the parts-per-billion level and detecting it requires highly sensitive observations at high spectral resolution together with very accurate spectroscopic parameters. This has motivated several studies to derive $\mathrm{CO}_{2}$-broadening line widths and $\mathrm{LM}$ effects in $\mathrm{CH}_{4}$ to prepare for upcoming observations (eg [453,454,501]). Despite the low surface pressure on Mars (600 $\mathrm{Pa}$ on average), $\mathrm{LM}$ effects are visible in $\mathrm{CH}_{4}$ spectra at the spectral resolution of the NOMAD spectrometer $\left(0.1 \mathrm{~cm}^{-1}\right)$ onboard ExoMars Trace Gas Orbiter [453] and they will be taken into account. Finally, since temperatures on Mars span the 150-290 K range, correct modeling of the effects of collisions with $\mathrm{CO}_{2}$ on individual lines and manifolds of all relevant absorbing species is needed below room temperature.

Regarding the lower atmosphere of Venus, where the pressure reaches $9 \mathrm{MPa}$ and the temperature $730 \mathrm{~K}$, experimental and theoretical efforts are still needed to correctly describe absorption in the far wings of $\mathrm{CO}_{2}$. Indeed, [625] evaluated several line-shape models (including a LM approach in the strong-collision approximation) that all failed to reproduce the observed $\mathrm{CO}_{2}$ absorption in the 1.10 and $1.18 \mu \mathrm{m}$ atmospheric windows. The authors thus derived their own empirical $\chi$-factor for the sub-Lorentzian $\mathrm{CO}_{2}$ line wings from fitting the spectra collected by the Spectroscopy for Investigation of Characteristics of the Atmosphere of Venus Infrared Red instrument (SPICAV_IR) (Fig. 21). In addition, to fit the observations, [625] and [626] also had to add an ad hoc continuum opacity, which probably results from collision-induced processes. Several recent high-pressure (but room temperature) CRDS experiments have been built to evaluate this continuum opacity and measure binary absorption coefficients in several transparency windows relevant to the atmosphere of Venus [551-554] (see also Sec. 4.4.2). In these experiments, there was also evidence of LM effects, which would have to be accounted for in future studies. As outlined in a recent review [627], additional theoretical models, predictions and experiments at high pressures but also high temperatures are needed to improve the existing databases of spectroscopic parameters for Venus conditions. 


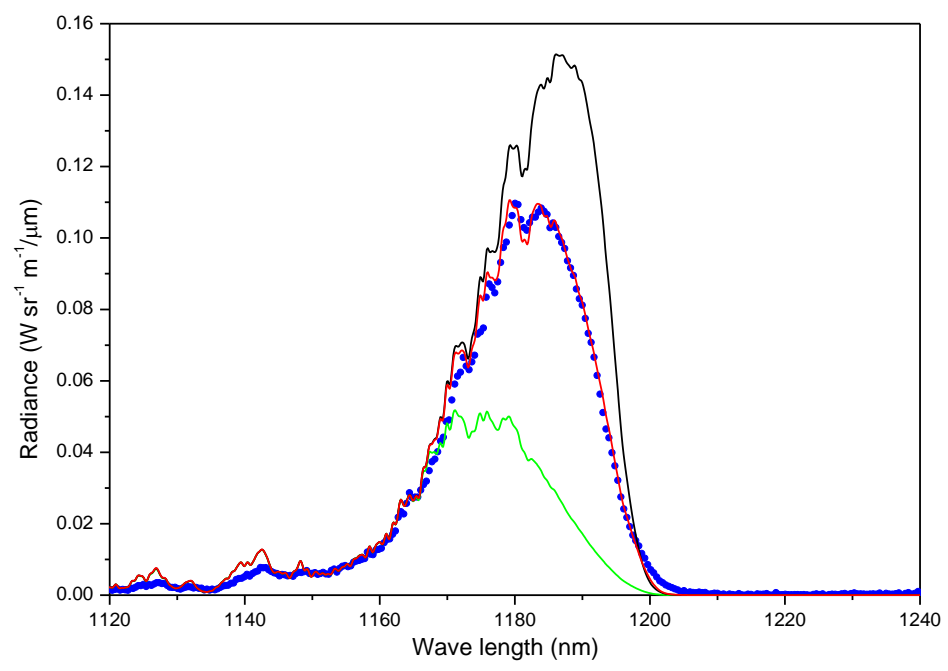

Fig. 21: The SPICAV-IR measured Venus emission spectrum (blue circles) and synthetic spectra calculated with different $\mathrm{CO}_{2}$ line shapes: fully Lorentzian with a cut-off at $250 \mathrm{~cm}^{-1}$ from line center(green line), Lorentzian corrected using a $\chi$ factor derived in the 2.3 um region [550] (black line), sub-Lorentzian profile proposed in [625] (red line). Courtesy of B. Bézard, after [625].

Titan, Saturn's largest satellite, has a nitrogen-dominated atmosphere (with $\sim 5 \%$ of $\mathrm{CH}_{4}$ and $\sim 0.1 \%$ of $\mathrm{H}_{2}$ ) with a surface pressure of $0.15 \mathrm{MPa}$. Many spectroscopic studies performed for the Earth's atmosphere are thus valid, provided that the spectral shape parameters are known (or can be extrapolated) down to below $100 \mathrm{~K}$. In the near-infrared, Titan's spectrum is dominated by methane absorption (and aerosol extinction). With accurate spectroscopic methane data, it is possible to see through the veil of Titan's opaque atmosphere in several spectroscopic windows and derive properties of Titan's surface albedo [628]. Given the importance of methane, a lot of work has been made (eg [629] in the past decade to complement the existing databases with line parameters. However, the analysis of ground- or space-based observations in the near-infrared is hampered by the (sub-Lorentzian) behavior of the methane line wings, which remains poorly known and plays an important role. Further work on methane line wings is clearly needed.

Regarding the atmospheres of giant planets, recent experiments have focused on measuring absorption properties of ammonia and water vapor under Jovian conditions that are found in its deep troposphere, ie in a hydrogen-helium atmosphere, up to $10 \mathrm{MPa}$ and $500 \mathrm{~K}$ (eg [630,631]) or even 600K [632], in support of a microwave remote sensing instrument onboard the Juno spacecraft. Such experiments are crucial to derive microwave absorption coefficients that are then used to retrieve the abundances of these species in Jupiter's deep troposphere $[633,634]$. Experiments have also been conducted to characterize the effects of collisions with $\mathrm{H}_{2}, \mathrm{He}$ as well as $\mathrm{H}_{2} \mathrm{O}$ [630] and $\mathrm{CH}_{4}$ [632] on the ammonia opacity in the 5 $\mathrm{cm}$ to $20 \mathrm{~cm}$ wavelength region. Based on these measurements, new models for pressurebroadened line shapes have been developed. These laboratory data and models show that broadening by water vapor has a measurable effect on the opacity of ammonia under Jovian conditions [630]. The broadening by $\mathrm{CH}_{4}$ is found to be considerably larger than those by $\mathrm{H}_{2}$ and He, but its effect on the microwave spectrum which will be observed by Juno is expected to be minimal, owing to methane's relatively low abundance on Jupiter ( $\sim 0.2 \%$ by volume) [632]. However, the influence of methane broadening on the ammonia absorption spectrum is expected to be more important on Uranus and Neptune where $\mathrm{CH}_{4}$ is of greater abundance ( $2 \%$ to $4 \%$ by volume).

Even more extreme environments can be found in the atmospheres of exoplanets or substellar objects such as brown dwarfs. Exoplanets observed with ground-based or space-based telescopes and for which molecular signatures can be detected in their atmospheres with 
current techniques are mainly "hot Jupiters" or "mini-Neptunes", with $\mathrm{H}_{2}$-rich atmospheres and temperatures in the range $500 \mathrm{~K}$ to $3000 \mathrm{~K}$. To adapt to such high temperatures, several molecular line lists have been developed in recent years, including the HITEMP [635] and the ExoMol $[636,637]$ databases. Both these databases provide a compilation of high-temperature line lists and line-broadening parameters for molecules such as $\mathrm{H}_{2} \mathrm{O}, \mathrm{CO}, \mathrm{CO}_{2}, \mathrm{CH}_{4}$ and $\mathrm{NH}_{3}$. However, the $\mathrm{H}_{2}$ pressure-broadening data are insufficient despite being critical to correctly model infrared spectra of brown dwarves, hot-Jupiter or mini-Neptune planets [638]. In [638], water vapor in a $\mathrm{H}_{2}$-rich exoplanetary atmosphere was used as a case study and synthetic transmittance spectra of water were computed at various spectral resolutions (from $10^{2}$ to $10^{5}$ ) and temperatures $(500 \mathrm{~K}$ to $3000 \mathrm{~K})$. The authors showed that neglecting pressure-broadening effects induces errors of up to $40 \%$ in calculated spectra at the medium resolution $(R=3000)$ representative of the future James Webb Space Telescope capabilities, with even higher errors (greater than $100 \%$ ) obtained at high spectral resolution. There is thus a strong need for accurate pressure-broadening data in the case of collisions with $\mathrm{H}_{2}$ up to very high temperatures. This is the topic of many recent theoretical studies. A comprehensive compilation of existing data can be found in a recent update of the ExoMol database, which to this date includes $\mathrm{H}_{2^{-}}$, He-, air- and self-broadening parameters relevant to eight molecules and high temperature ( $c f[637,639]$ and references therein).

\subsubsection{Probing combustion gases}

Laser techniques have been used for decades for the probing of combustion gases. A variety of gas-phase systems, which involve wide ranges of pressure, temperature and composition, have been probed for steady-state and time-resolved determinations of temperature, pressure, velocity, relative amounts of molecular species, etc. In most cases the accuracy of the retrieved results highly depends on the quality of the spectroscopic data and spectral shapes used. On this topic, the reader is invited to consult several recent reviews [640-642] and the references contained therein.

\subsubsection{Probing confined gases}

It was predicted many years ago that the (tight) confinement of molecular gases inside enclosures where they are free to move (and not adsorbed) induces a modification of the shapes of absorption lines through the increased contribution of molecule-surface collisions $[643,644]$. However, not until around 2010 did measurements clearly demonstrate these confinement effects [645-647]. These experiments showed that lines of $\mathrm{H}_{2} \mathrm{O}$ vapor inside an aerogel sample [645] and of $\mathrm{O}_{2}$ gas inside ceramics [647] are significantly broader than those observed for the same pressures and species under unconfined conditions. This is also shown in $[648,649]$ and Fig. 22, where the broad feature caused by the confinement of CO gas in an aerogel sample, is more than 30 times broader than the narrow peak which is its counterpart in the free gas phase. 


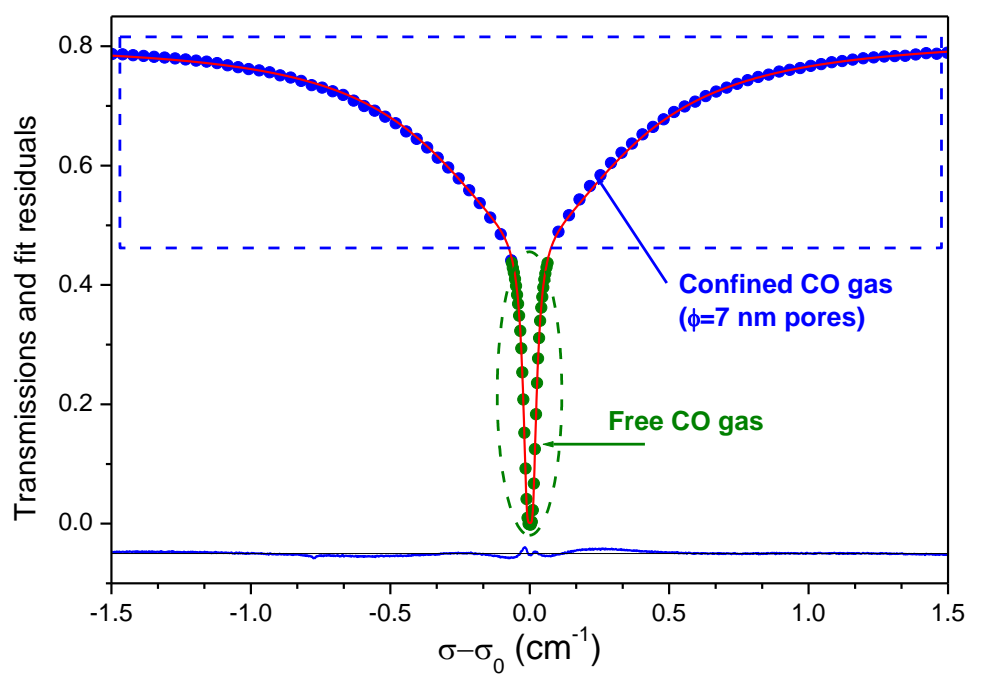

Fig. 22: Transmission spectra around the $R(6)$ line of the fundamental band of $\mathrm{CO}$ at $\mathrm{P}=117$ hPa for a path including a porous xerogel sample and space between the edges of this sample and the cell windows. The symbols (•, not all plotted) are measured values with blue and green colors indicating the contributions of the confined and free gas, respectively. The red and blue lines are the fitted transmission and the (shifted by-0.05) meas-fit residuals. After [649]

These findings led to the conclusion that the observed line widths of confined gases can be used to measure the size of microscopic pores [650]. The relation between broadening and confinement [644] was then confirmed by both CMDS [119,651] and experiments under wellcontrolled confinement conditions [652]. This established the applicability of line-width measurements to porosimetry. Indeed, it was recently shown that infrared absorption by gases within the pores of several samples gives reliable information on the percentage and dimensions of open pores [649,653]. Spectroscopy thus appears [649] as an interesting tool complementary to more commonly-used techniques such as mercury intrusion/extrusion or $\mathrm{N}_{2}$ sorption/desorption isotherms. Note that, besides line-broadening, spectra of molecular gases within porous samples have also been analyzed from other points-of-view, including the effects of molecule-surface collisions on LMeffects [334] and induced absorption [654,655]. Finally, let us mention studies $[656,657]$ in which the absorption in the $v_{3}$ band of $\mathrm{CO}_{2}$ was used to probe nano-bubbles of this gas formed at the interface between water and a hydrophobic solid.

\subsection{Metrology}

\subsubsection{Doppler thermometry}

In the last decade, significant efforts have been devoted worldwide to the determination of the Boltzmann constant $\left(k_{\mathrm{B}}\right)$ with the aim of redefining the Kelvin unit [658]. Indeed, the new SI and its implementation will be formally adopted by the end of 2018. This includes the Kelvin which will be redefined as the change of thermodynamic temperature $\Delta T$ resulting in a change of thermal energy by $k_{\mathrm{B}} \Delta T$, thus implying that the Boltzmann constant should be known as accurately as possible. For this reason, several primary thermometry methods, based upon the measurement of a thermodynamic property of a macroscopic system at the temperature of the triple point of water, have been improved enormously in the last few years [659], yielding relative uncertainties in $k_{\mathrm{B}}$ at the parts-per-million level. Doppler broadening thermometry (DBT) was proposed as an optical technique [660] to determine $k_{\mathrm{B}}$ from measurement of the Doppler width $\Delta v_{\mathrm{D}}$ of an absorption line of a gas. Indeed, $k_{\mathrm{B}}$ and $\Delta v_{\mathrm{D}}$ are related by $\Delta v_{\mathrm{D}}=\left(v_{0} / c\right) \sqrt{2 \ln 2 k_{\mathrm{B}} T / M}$, where $v_{0}$ is the central frequency of the line, $M$ is the absorber (atomic or molecular) mass and $T$ is the temperature. Inverting this equation thus leads to the determination of the thermal energy and, consequently, of either the gas temperature or the Boltzmann constant. 
After the first successful experiment [661], the international community of fundamental metrology widely recognized the importance of developing an optical method that links the thermodynamic temperature to an optical frequency, to obtain an independent confirmation of the results provided by more established methods. So far, the most accurate implementation of DBT has been performed on a line of $\mathrm{H}_{2}{ }^{18} \mathrm{O}$ vapor at $1.39 \mu \mathrm{m}$ [662]. Using the dual-laser spectrometer described in Sec. 2.8 and a sophisticated spectral analysis procedure, $k_{\mathrm{B}}$ was determined with a combined uncertainty of 24 parts-per million (ppm) [663].

While the current status and new frontiers of research of DBT have been reviewed in [162], it is noteworthy that, in addition to the quality of the detection system, the line-shape model used for the spectral analysis and retrieval of $\Delta v_{\mathrm{D}}$ from measured spectra is a key issue, as discussed in [664]. In the first determinations, the spectral analysis was performed by using either a Gaussian or a Voigt profile $[62,661,665]$. Subsequently, improyements of the experimental setups have been accompanied by using increasingly refined line shapes. Great attention has been paid to the roles of velocity-changing collisions and speed-dependence of relaxation rates [469,666-668]. Both effects have been accounted for in [662], in which a partially correlated speed-dependent hard-collision model [171] was used. In order to go further, a global (or multispectrum) analysis approach was then adopted for a simultaneous fit to a manifold of experimental profiles across various pressures. As explained in [669], this global analysis should reduce the uncertainty associated with the line-shape model, as well as the fluctuations resulting from statistical correlations among the adjusted parameters. Another way to reduce the uncertainty caused by the approximate modeling of collisional effects (ie the line-shape model) is to use a highly sensitive detection technique capable of probing samples at very low pressures. This is possible by using long-path absorption techniques or cavity-enhanced methods, as recently done in [670] where acetylene spectra near $787 \mathrm{~nm}$ were recorded using CRDS at pressures as low as $1.5 \mathrm{~Pa}$. Nevertheless, large systematic deviations (of about $800 \mathrm{ppm}$ ) remained, mostly ascribed to weak hidden lines overlapping with the selected transition. Very recently, a comb-assisted highly sensitive CRDS spectrometer, operating at $1.578 \mu \mathrm{m}$, has been developed [671] using pure $\mathrm{CO}_{2}$ as a molecular gas target since, as discussed in [162], this molecule turns out to be an excellent choice for DBT measurements. It was subsequently shown that the combination of high sensitivity, absolute frequency calibration and extremely dense sampling of the absorption profiles leads to a statistical uncertainty of $8 \mathrm{ppm}$ over a measurement time of only $5 \mathrm{~h}$. Thermodymanic temperature determinations have been demonstrated by using this approach, with the spectroscopically unprecedented combined uncertainty of 14 ppm [671].

\subsubsection{Amount of gas metrology}

Linear absorption spectroscopy of isolated transitions is a versatile and potentially absolute method for measuring the gas-phase amount of a substance ( $c f$ Sec. 5.1) that can be modeled by the Beer-Lambert law. This approach is species- and isotopologue-specific (contrary to mass spectrometry), linear in concentration (assuming that saturation effects are negligible), and suitable for both inert and reactive gases. The line intensity plays the role of an intrinsic molecular standard provided this quantity and the line shape (a key issue as discussed below) are accurately known, thereby supplanting the routine need for calibration gases. Also, ratios of spectral-peak areas can be used to measure the relative abundance of isotopologues in terms of known line intensities, without the need for an arbitrary reference material as required in mass spectrometry.

Concerning the line intensity, it can be computed using ab initio predictions of the wave functions and dipole-moment surfaces (DMSs) $(e g$ [672,673]) or it can be determined from effective DMSs and Hamiltonians that have been fitted to numerous measured values with one goal being to average over independent biases among experiments (eg [674]). Laboratory 
measurements of line intensities are potentially more accurate than predictions and, in any case useful for assessing uncertainties. However, except for rotational transitions in the far infrared for which Stark and Zeeman measurements can be made, accurate experimental determinations of line intensities must meet several technical challenges. The first, for which possible solutions are discussed in Sec. 2, is that high-fidelity measurements of both the spectrum detuning and absorbance axes are required. The second is that the composition, pressure (or number density) and temperature of the gas sample introduced in the spectrometer must be stable and accurately known. This can be solved by using accurate and precise $P$ and $T$ sensors and certified mixtures that are commercially available for numerous gases or which can be prepared using various SI-traceable methods ( $e g$ [675-678]). For the most stable gases, mixtures with relative standard uncertainties of less than $0.1 \%$ are generally available. Note that, particularly when $\mathrm{H}_{2} \mathrm{O}$ is involved, steady flows of humidified gas conditions are preferable over cylinder-based sources which tend to exhibit long-term drift $[435,679]$. Finally, to retrieve the line intensity from fits of measured spectra the normalized line profile used must be carefully chosen. The profile enables optimal exploitation of the experimental information, in addition to enabling interpolation between data points and ensuring the extrapolation necessary to account for the peak area underneath the line wings outside of the measurement domain. From this point of view it has been shown that using the VP leads to systematic and pressure-dependent errors (up to several \%) on the retrieved line intensity that are usually larger than experimental uncertainties $[46,188,284,435,467,489,680,681]$. Furthermore, the fitted results are much more sensitive to the spectral range considered [435] by comparison to results obtained with higher-order line profiles. However, when using more advanced profiles involving several floated parameters (speed-dependence and/or velocity-changing frequency, etc), differences between fitted areas decrease strongly and may become as small as $0.05 \%$ (eg [46,682,683]). Given the availability of low-uncertainty SI-traceable gas standards and the capability to measure spectra with SNRs far greater than 1000 and analyze them with advanced line-shapes, the last decade has seen the emergence of absolute line-intensity measurements with relative uncertainties approaching $0.1 \%[8,60,96,683,684]$.

Obviously, when the line intensity is assumed known in order to determine the relative or absolute density of a given molecule (or isotopologue) from an absorption measurement, the use of an improper line shape in the fits leads to errors on the retrieved value. Discussions on this issue in the context of isotopic-ratio determinations can be found in [680,685-687]. In particular, Fig. 23 shows the measured spectra and associated fit residuals used [687] to retrieve the $\mathrm{H}_{2}{ }^{17} \mathrm{O} / \mathrm{H}_{2}{ }^{16} \mathrm{O}$ amount ratio. While the VP is clearly inadequate, the GP, NelkinGhatak profile (NGP or RP) and SDVP lead to comparable and much smaller residuals. As for the ${ }^{17} \mathrm{O} /{ }^{16} \mathrm{O}$ ratio, that obtained with the VP is unrealistically large ( $\left.\approx 581 \mathrm{ppm}\right)$, while the GP and SDVP provide the same results which are about $0.8 \%$ smaller than those obtained with the NGP $(\approx 368 \mathrm{ppm})$ [687]. It should be noted that the literature on isotopic-ratio measurements by means of laser spectroscopy, as an alternative to mass spectrometry, is quite broad. An exhaustive coverage of this field would be outside the scope of the present review article, also considering that in many cases a refined line-shape model is not required [688]. 


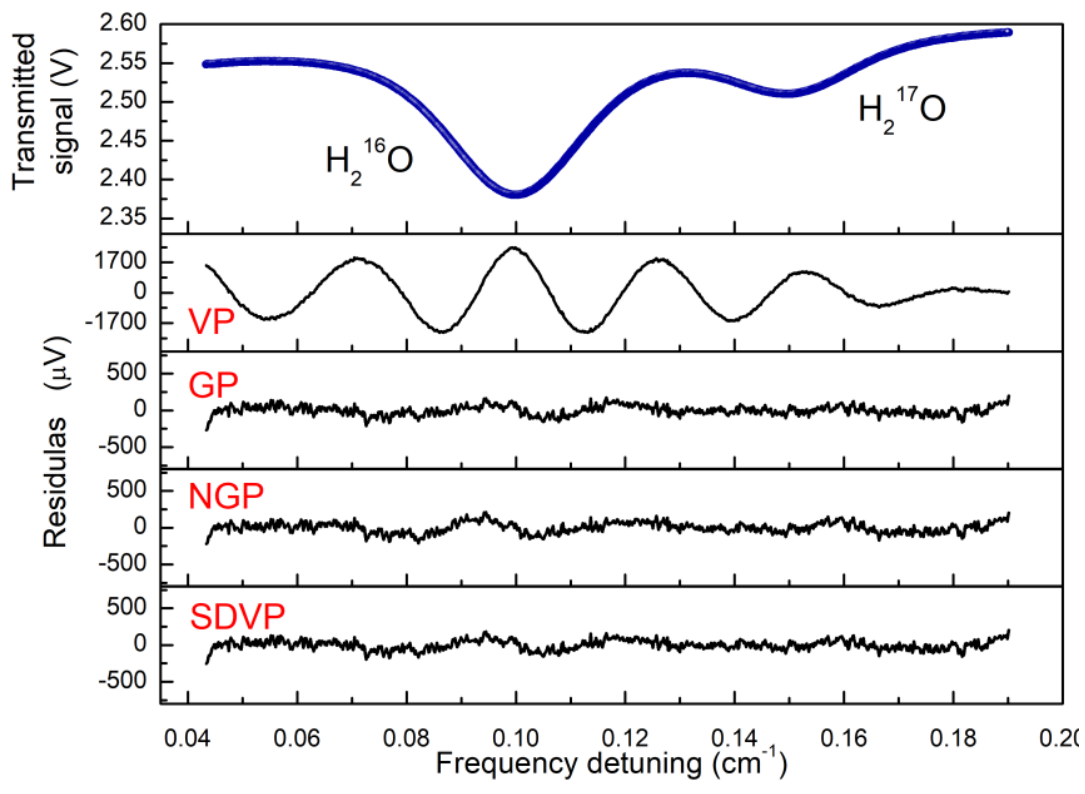

Fig. 23: Experimental water vapor spectrum near $\lambda=1392.0625 \mathrm{~nm}$ for a total pressure of about $2000 \mathrm{~Pa}$ (15 Torr), at a temperature of 298.15 K. Absolute residuals are also reported, resulting from the non-linear leastsquares fit to Voigt, Galatry, Nelkin-Ghatak, and speeddependent Voigt profiles. The $\mathrm{H}_{2}{ }^{16} \mathrm{O}$ peak corresponds to the $6_{3,3} \rightarrow 5_{2,4}$ component of the $2 v_{3}$ band, while the $\mathrm{H}_{2}{ }^{17} \mathrm{O}$ peak is due to the $2_{1,1} \rightarrow 1_{1,0}$ line of the $v_{1}+v_{3}$ band. After [687].

Another particularly important field requiring accurate line-intensity data involves the remote sensing of greenhouse gases from satellite and terrestrial platforms Examples include NASA's OCO-2 satellite [577,585,588], ground-based TCCON [594] observations of $\mathrm{O}_{2}$ and $\mathrm{CO}_{2}$, and the planned MERLIN satellite for measuring $\mathrm{CH}_{4}$ [578]. These applications have concentration retrieval targets with uncertainties at the $0.25 \%$ level or below and therefore motivate low-uncertainty laboratory measurements that incorporate advanced line profiles (eg $[9,100,689]$ and references therein ; see also Sec. 5.1.1a).

\subsection{Radiative heat transfer and climate modeling}

In radiative heat transfer problems, one generally wishes to predict the power locally lost or gained by the gas $\left(e g\right.$ in $\left.\mathrm{W} / \mathrm{m}^{3}\right)$ and the local net fluxes $\left(e g\right.$ in $\left.\mathrm{W} / \mathrm{m}^{2}\right)$ to solid surfaces. This is obviously relevant for industrial systems where these quantities may affect the hydrodynamics and efficiencies of combustion processes, as well as the longevity of the solid parts of the system. It is also crucial for correct predictions of climate and radiative forcing, with the example of predicting the degree to which warming of the Earth can be attributed to anthropogenic greenhouse gases. For such problems, the quantities of interest are integrated over the entire thermal emission spectrum of the medium and, eventually, also over that of an external source of radiative energy ( $e g$ the Sun). Because of this broad integration, the results are generally almost insensitive to refined pressure effects affecting narrow spectral intervals ( $e g$ the deviations from the VP in the core regions of the lines discussed in Sec. 2.2). However, this may not be the case at (very) elevated pressures for which LM can significantly change the shape of entire bands after the line structure has vanished. In addition, the far wings of lines and CIA may then play a non negligible role, particularly when they affect the regions of relative transparency between the strongly absorbing bands.

\subsubsection{Heat transfer in "industrial" devices}

In systems of human scale, the path lengths and gas densities are generally too low to make far wings and CIA significant. Furthermore, when at elevated pressure, gases in industrial devices are often also at high temperature. The resulting densities are then moderate so that LM effects in the central regions of the bands remain small. These elements, and the computer-cost inherent to the use of sophisticated line-shapes, explain why radiative transfer calculations for industrial-type applications mostly use line-by-line approaches with VPs 
(eventually corrected by a $\chi$ factor in the wings), or even simpler models built from them. [690-692] present such simplified approaches and examples of their use in heat-transfer calculations can be found in papers cited therein as well as in studies among those that cite these three references.

\subsubsection{Modeling atmospheres observed in the solar system}

High pressure atmospheres are found on the giant planets Jupiter, Saturn, Uranus and Neptune $\left(\mathrm{H}_{2}\right.$ and $\left.\mathrm{He}\right)$, Venus $\left(9 \mathrm{MPa}, 95 \% \mathrm{CO}_{2}\right)$ and, to some extent, Titan $(0.15 \mathrm{MPa}, 95 \%$ $\mathrm{N}_{2}$ ). The past ten years have seen an increased interest in the accurate numerical simulation of radiative transfer within high-pressure planetary atmospheres with the development of a new generation of Global Climate Models (GCM) for most solar system atmospheres, supported by space missions equipped with atmospheric sounders like Venus Express, Cassini (Saturn, Titan) and Juno (Jupiter).

Among the terrestrial planets, Venus remains challenging because of its high-pressure and high-temperature atmosphere. The available spectroscopic data for individual lines, complemented by the high-pressure $\mathrm{CO}_{2}$ data and the $\mathrm{H}_{2} \mathrm{O}$ continuum parameterizations discussed in Sec. 4.3, have allowed the main behavior of the observed temperature profiles to be reproduced using physical models (eg [693-695]). The thermal structure is found to be primarily controlled by energy exchanges taking place in the infrared, especially in windows where the opacities are smallest, and thus not well known. Specifically, [694] showed that to model the Venusian atmosphere, uncertainties in the air opacity are crucial in two spectral regions which remain poorly known: (i) Between $20 \mu \mathrm{m}$ and $30 \mu \mathrm{m}$, in the window between the $15 \mu \mathrm{m} \mathrm{CO}$ band and the $\mathrm{CO}_{2}$ roto-translational CIA. Light-matter interactions in these spectral regions control the temperature in the cloud convective layer (45 km to $60 \mathrm{~km}$ ). (ii) In the infrared region, energy exchange between the deep atmosphere, the cloud base and within the deep atmosphere occurs mostly in the $3 \mu \mathrm{m}$ to $4 \mu \mathrm{m}$ and $5 \mu \mathrm{m}$ to $7 \mu \mathrm{m}$ intervals. The calculated thermal structure was also found to depend in the solar energy deposited below the clouds and thus on the assumed cloud properties, which can be adjusted. Errors in the thermal radiative transfer can be partly compensated, and it is therefore not straightforward to constrain the gaseous opacities when fitting the observed atmospheric temperatures. Nevertheless, as reported in [694] it was concluded that, when using the latest data on $\mathrm{CO}_{2}$ and $\mathrm{H}_{2} \mathrm{O}$ absorption in the $3 \mu \mathrm{m}$ to $7 \mu \mathrm{m}$ spectral region, the opacity is not quite enough to reach the observed surface temperatures in the model when the cloud-base temperature is consistent with observations: "additional laboratory measurements of $\mathrm{CO}_{2}$ absorption in the conditions of the deep atmosphere of Venus (1-10 MPa, $500 \mathrm{~K}$ to $750 \mathrm{~K})$ are needed".

Titan is, in theory, easier to model than Venus since, like the Earth, it has a nitrogendominated atmosphere (with $\sim 5 \%$ of $\mathrm{CH}_{4}$ and $\sim 0.1 \%$ of $\mathrm{H}_{2}$ ) with a surface pressure of only $0.15 \mathrm{MPa}$. Except for the very low temperatures $(<100 \mathrm{~K})$ of the troposphere and lower stratosphere, for which measurements and/or calculations are still needed, many spectroscopic studies performed for the Earth's atmosphere are thus valid. Nevertheless, CIA plays a stronger role on Titan than on the Earth, with contributions from $\mathrm{N}_{2}-\mathrm{N}_{2}, \mathrm{~N}_{2}-\mathrm{CH}_{4}, \mathrm{~N}_{2}-\mathrm{H}_{2}$ and $\mathrm{CH}_{4}-\mathrm{CH}_{4}$. Nowadays the relevant data can be obtained from HITRAN [432,433], which are notably based on the relatively old studies of [696-698]. Radiative heating occurs through the absorption of visible and near-infrared light by methane and aerosols. As mentioned in section 5.1.1b, methane spectroscopy beyond $0.8 \mu \mathrm{m}$ has been improved recently [629] but a remaining issue is related to the (sub-Lorentzian) behavior of the methane line wings, which remains poorly known. It is currently derived [628] from observations of the Titan atmosphere by the Cassini Visual and Infrared Mapping Spectrometer (VIMS) in the near infrared. 
In giant-planet atmospheres, radiative cooling primarily occurs through the thermal emission of minor species (eg methane, ethane, acetylene) along with CIA by $\mathrm{H}_{2}-\mathrm{H}_{2}$ and $\mathrm{H}_{2}-$ $\mathrm{He}$ in the thermal infrared (see Sec. 5.1.1b for further details and [699] for a recent work on Saturn).

\subsubsection{New challenges: simulating exoplanets and ancient atmospheres}

More and more studies are conducted to model "new" kinds of atmospheres which are thought to have existed in the past in the solar system, or to be present on extrasolar planets. Within this context, the need for a good understanding of the effect of pressure on the spectra of gases in a wide range of conditions is stronger than ever. The number of possible cases is countless. Indeed, while the atmospheres of most extrasolar planets have not been characterized yet, it is known that the diversity of exoplanets is huge, including giant planets, mini-Neptunes, volatile rich rocky planets, in all possible ranges of temperatures (eg [700]). For both the ancient solar system planets and the exoplanets, there is a special interest in the modeling of the conditions that control habitability at the surface (ie allowing the existence of liquid-water lakes or oceans) and the limit of the "habitable zone". Such studies usually involve high-pressure atmospheres and challenging spectroscopic issues.

On strongly irradiated planets (early Venus or exoplanets near the inner edge of the habitable zone), a key concern is the greenhouse effect of warm $\mathrm{H}_{2} \mathrm{O}$-rich atmospheres and the uncertainties in the water-vapor continuum which controls the "runaway greenhouse effect" that defines the limit of habitability [529-531,701].

On the opposite, in order to investigate the possible climates on weakly irradiated terrestrial planets (early Mars or exoplanets near the outer edge of the "habitable zone") most studies have focused on the radiative effect of 0.1 to $1 \mathrm{MPa} \mathrm{CO}_{2}$-rich atmospheres. Interestingly, such atmospheres have been found difficult to model, and many problems have arisen. For instance, until 2010, to calculate the far infrared collision-induced opacity in a multi-bar $\mathrm{CO}_{2}$ atmosphere, almost all published studies (eg [701-704]) relied without modification on a parameterization originally derived for the Venus atmosphere in [705] (see detailed description in [706]). It was based on the measurements of [707] from $7 \mathrm{~cm}^{-1}$ to 250 $\mathrm{cm}^{-1}$, and on a simple parameterization of collision-induced opacity in the other spectral domains described in an unpublished $\mathrm{PhD}$ thesis [708], still available as a NASA report. This parameterization included significant opacities between $295 \mathrm{~cm}^{-1}$ and $526 \mathrm{~cm}^{-1}$ actually resulting from the pressure-broadened wings of the $15 \mu \mathrm{m}$ bands! Unfortunately, this feature was kept in subsequent models, despite the fact that these opacities were probably overestimated and, in most cases, already accounted for in the codes chosen to calculate the radiative transfer in the $15 \mu \mathrm{m}$ band. As a result, all studies overestimated the greenhouse warming of $\mathrm{CO}_{2}$ by several $\mathrm{K}$. [709] later combined the results of [415] and [710] to propose a more up-to-date method to account for the CIA when using modern spectroscopic databases for $\mathrm{CO}_{2}$.

More recently [711] claimed that explicitly including the effect of $\mathrm{CO}_{2}$ collisional LM in their radiative transfer calculations yielded $\mathrm{CO}_{2}$ atmospheres that are more transparent to infrared radiation than when spectra calculations are made using $\chi$-corrected Lorentzian line shapes, thus generating much colder temperatures than previously estimated. However [712] later showed that this relative cooling was rather due to the wrong choice of broadening species (air instead of $\mathrm{CO}_{2}$ ) and that when using properties derived for self-broadened $\mathrm{CO}_{2}$ [311] no significant change could be noticed. Understanding why the early Martian climate could have been warm enough for liquid water to flow on the surface remains one of the major enigmas of planetary science [713] and no consensus scenario has yet been reached. The solution certainly lies in experiments for long paths and/or elevated gas pressures, capable of probing spectral regions where the (weak) absorption is proportional to the squared 
total density. For instance, the most promising recent theory is the study from [714] where it was suggested that methane or hydrogen could have acted as powerful greenhouse gases in the early Martian atmosphere because of $\mathrm{CH}_{4}-\mathrm{CO}_{2}$ and $\mathrm{H}_{2}-\mathrm{CO}_{2}$ CIA. In atmospheres of 50 $\mathrm{kPa} \mathrm{CO}$ or more, percent levels of $\mathrm{H}_{2}$ or $\mathrm{CH}_{4}$ were shown to raise the annual mean surface temperatures by tens of $\mathrm{K}$, with temperatures reaching $273 \mathrm{~K}$ for pressures of $0.125 \mathrm{MPa}$ to $0.2 \mathrm{MPa}$ and $2 \%$ to $10 \%$ of $\mathrm{H}_{2}$ and $\mathrm{CH}_{4}$. Such solutions had been previously explored [715], but the CIA-induced greenhouse effect was probably underestimated because of the use of CIA data adapted for an $\mathrm{N}_{2}$ atmosphere rather than a $\mathrm{CO}_{2}$ atmosphere. In fact, the coefficients used by [714] remain theoretical and measurements are urgently needed.

The same conclusions can be reached for many of the ongoing climate studies in planetary science. In particular, even for heavily studied molecules like $\mathrm{CO}_{2}$ or $\mathrm{H}_{2} \mathrm{O}$, much remains to model the extreme environment on some exotic exoplanets [700]), in protoatmospheres [716] or terrestrial atmospheres that are subject to asteroid impacts [717].

\section{Remaining issues and directions for future research}

This review shows that considerable progress has been made in the last decade in the determination of spectral-shape parameters from laboratory measurements. These advances, particularly significant for isolated lines, result from the unprecedented accuracy of modern experiments and the analysis of measurements with multispectrum fitting techniques using refined models. In many cases, the absorption can now be measured and represented in a broad pressure range with fit residuals of a few $0.1 \%$ or lower. At the same time, promising theoretical approaches have been proposed for the prediction of pressure effects from the intermolecular interaction potential. Together with more empirical approaches in which some parameters are adjusted, these capabilities often enable accurate descriptions of numerous mechanisms affecting the spectral shape, from individual lines to entire (allowed or collision induced) absorption bands. Despite these achievements, many issues remain, from both the experimental and theoretical points of view, in conjunction with the needs of practical applications or of our understanding of physical processes. These remaining issues are far too numerous to be all discussed here, but we below discuss some possible directions for future research.

\subsection{Experiments}

Despite considerable progress, current laboratory experimental set-ups remain limited for the following reasons: (i) The first is that the accessible temperatures are often limited to room temperature or, when variable, within typically $220 \mathrm{~K}$ to $350 \mathrm{~K}$. Furthermore, when the sample temperature can be varied, the path length is generally quite limited. (ii) In addition, only sub-atmospheric pressures can often be studied. (iii) Finally, tunable-laser experiments, which provide the most accurate and sensitive measurements, are limited to relatively narrow spectral intervals and low generated-data throughputs. These limitations are generally not too constraining with regard to applications in radiative transfer and remote sensing in the atmosphere of the Earth, but this is not the case for many planetary studies. Indeed, exoplanets or early (paleo) objects in the solar system often involve high temperatures and/or pressures together with atmospheres that are extremely optically thick in the central regions of many bands. The absorption in the windows of relative transparency must then be accurately modeled and providing the relevant quantitative information may require measurements under conditions that are not yet obtainable in the laboratory. There is thus a need to develop experimental set-ups in which absorption for very large optical paths at elevated pressure and temperature can be investigated. Considering the extension of spectral ranges that can be 
studied, we are relatively confident that it will broaden with the development of new laser sources and high reflectivity mirrors.

As it appears from the preceding sections, a relatively limited number of spectra have been recorded over broad temperature intervals. Consequently, the available comparisons between measurements and predictions remain often insufficient for a thorough assessment of the quality of some theories or models. For the same reason, the temperature dependence is known for relatively few of the spectral-shape parameters deduced from fits of measured spectra. We thus believe that efforts should be made both for the development of new set-ups and the use of existing ones for the investigation of pressure effects over extended temperature ranges. In doing this, as recalled at the end of Sec. 2.1, much care should be taken for the precise determination of the characteristics of the gas sample (and particularly its temperature and pressure) so that associated uncertainties do not degrade the quality of the results.

\subsection{Theories and models}

With regard to theories, the modified Robert-Bonamy approach, the use of molecular dynamics simulations and some recent quantum mechanical approaches for CIA are, in our opinion, the main recent advances. Their extensions to other molecular systems are obviously relevant for future studies, and their ability to describe the effects of temperature, which was seldom studied so far, must be further tested. Together with the other approaches also presented in Sec. 3, they provide a broad set of tools for predictions of the various collisional effects influencing the absorption shapes. However, although the predicted trends afforded by these models are quite good in most cases, absolute predictions (ie those achieved without adjustment of any parameter) remain significantly less reliable than laboratory measurements. In addition, they also generally do not fulfill the accuracy required by various applications. From this point of view, we are quite pessimistic regarding the question of whether theories will be able to catch up with experiments and predict spectra at the typical uncertainty of $0.1 \%$. However, there are two reasons why predictive theories should not be disregarded. First, for the situations where no measurements are available or feasible, theory is the only option. It is, for instance, the case of the far infrared CIA by $\mathrm{CO}_{2}+\mathrm{H}_{2} \mathrm{O}$ mixtures, needed for planetary studies (Sec. 5.3.3), which is extremely difficult (if not impossible) to measure but can be predicted by CMDS (Sec. 3.6.1). Other examples are given by spectral-shape parameters at very high temperature, which can only be obtained (eg [207,404]) from calculations. Second, theories can be used to identify and quantify the respective contributions of the physical processes involved in the spectral-shape issue under consideration. This provides a tool to develop empirical or phenomenological models, helping to identify which are the key parameters and their behaviors. For instance, calculations using the approaches described in Sec. 3.3, or fits of CMDS calculated spectra (Sec. 3.2.1) with the HTP (Sec. 3.2.4a), may provide information on how to parameterize the influence of temperature on the speed-dependence and narrowing parameters. In addition, theories can bring valuable information to help in the analysis of experiments by identifying which are the most influential processes and parameters. For instance, the modified RB formalism (Sec. 3.4.3) can indicate which lines are significantly affected by LM in order to reduce the number of unknowns in fits to measured spectra. Predictions can also be used to constrain those parameters used in sophisticated profiles that cannot be reliably fitted because of the noise on measured spectra and/or of the limited pressure range investigated, and/or because of the large numbers of known and unknown variables, as well as the poorly defined correlations among them.

\subsection{Databases}


The current version of the HITRAN database [430] provides parameters for the HTP (and less refined non-Voigt line-shapes) only for some transitions of a limited set of molecules, due to the relatively recent incorporation of this line shape into the analysis of experiments. As new measurements become available they will undergo thorough evaluation before inclusion into the database. In addition, only first-order (Rosenkranz) LM parameters are currently provided (except for $\mathrm{CO}_{2}$ ). This is mostly because the various multi-spectrum fitting softwares employed in laboratories worldwide use slightly different formalisms when fitting LM along with correlated parameters. This situation makes it difficult to deliver consistent LM data to users. Ideally one should be able to provide codes (for instance within HAPI) for users to calculate absorption coefficients themselves from archived parameters. What constitutes the most efficient solution for this problem is still an open question under discussion in the spectroscopic and atmospheric communities. The number of planetaryrelevant molecules that have broadening and shift parameters caused by collisions with $\mathrm{CO}_{2}$, $\mathrm{H}_{2}$ and $\mathrm{He}$ needs to be extended. Similarly, data for line-broadening by water vapor of some important atmospheric gases will also have to be introduced. For all these topics there is a clear need for more information concerning the effects of temperature. Furthermore, for linemixing and some of the parameters (the speed dependence, the Dicke narrowing, etc) of refined individual line shapes, a consensus is still to be found on how to parameterize their temperature dependences. This also stands for the usual widths and shifts, for which the use of the single $296 \mathrm{~K}$ reference to parameterize their variations with $T$ needs to be questioned if one wants to provide data suitable for very broad temperature ranges. Finally, note that there is an effort underway [433] to substantially update the CIA section of the database described in [432].

\section{Acknowledgements}

The authors thank R. Ciurylo for fruitful comments that helped to improve this paper, and T. Karman and B. Bézard for providing some figures. M. Gustafsson gratefully acknowledges the support from the Knut and Alice Wallenberg Foundation. Part of this research was performed at the jet Propulsion Laboratory, California Institute of Technology, under contract with NASA. The HITRAN database is currently supported by NASA grants from AURA and PDART programs. The GEISA database is grateful to the Centre National d'Etudes Spatiales (CNES) for its constant support over the years. J.T. Hodges acknowledges support from the NIST Greenhouse Gas Measurement and Climate Research Program and from NASA contract NNH15AZ96I (Orbiting Carbon Observatory-2 Science Team). D. Lisak was supported by the National Science Center, Poland, project No. 2015/18/E/ST2/00585.

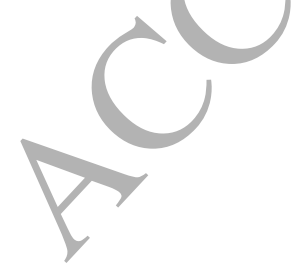




\section{References}

[1] Hartmann JM, Boulet C, Robert D. Collisional effects on molecular spectra: Laboratory experiments and models, consequences for applications. Elsevier, Amsterdam, (2008).

[2] Petersen M, Viallon J, Moussay P, Wielgosz RI. Relative measurements of ozone absorption crosssections at three wavelengths in the Hartley band using a well-defined UV laser beam. J Geophys Res Atmos 2012;117:D05301.

[3] Hodges JT, Layer HP, Miller WW, Scace GE. Frequency-stabilized single-mode cavity ring-down apparatus for high-resolution absorption spectroscopy. Rev Sci Instruments 2004;75:849-63.

[4] Long DA, Cygan A, van Zee RD, Okumura M, Miller CE, Lisak D, Hodges JT. Frequency-stabilized cavity ring-down spectroscopy. Chem Phys Lett 2012;536:1-8.

[5] Burkart J, Romanini D, Kassi S. Optical feedback frequency stabilized cavity ring-down spectroscopy. Opt Lett 2014;39:4695-8.

[6] Burkart J, Kassi S. Absorption line metrology by optical feedback frequency-stabilized cavity ringdown spectroscopy. Appl Phys B 2015;119:97-109.

[7] Domysławska J, Wójtewicz S, Lisak D, Cygan A, Ozimek F, Stec K, Radzewicz Cz, Trawiński RS, Ciuryło R. Cavity ring-down spectroscopy of the oxygen B-band with absolute frequency reference to the optical frequency comb. J Chem Phys 2012;136:024201.

[8] Sironneau VT, Hodges JT. Line shapes, positions and intensities of water transitions near $1.28 \mu \mathrm{m}$. J Quant Spectrosc Radiat Transf 2015;152:1-15.

[9] Delahaye T, Maxwell SE, Reed ZD, Lin H, Hodges JT, Sung K, Devi VM, Warneke T, Spietz P, Tran $\mathrm{H}$. Precise methane absorption measurements in the $1.64 \mu \mathrm{m}$ spectral region for the MERLIN mission. $\mathrm{J}$ Geophys Res Atmos 2016;121:7360-70.

[10] Reed ZD, Hodges JT. Line shape parameters of helium-broadened ${ }^{12} \mathrm{C}^{16} \mathrm{O}$ in the $3 \rightarrow 0$ overtone transition near 1.57 $\mu \mathrm{m}$. J Quant Spectrosc Radiat Transf 2017;203:300-8.

[11] Cygan A, Lisak D, Wojtewicz S, Domyslawska J, Hodges JT, Trawinski RS, Ciurylo R. High signal to noise ratio laser technique for accurate measurements of spectral line parameters. Phys Rev A 2012;85:022508.

[12] Lin H, Reed ZD, Sironneau VT, Hodges JT. Cavity ring-down spectrometer for high-fidelity molecular absorption measurements. J Quant Spectrosc Radiat Transf 2015;161:11-20.

[13] Cygan A, Wcisło P, Wójtewicz S, Masłowski P, Hødges JT, Ciuryło R, Lisak D. One-dimensional frequency-based spectroscopy. Opt Express 2015;23:14472-86.

[14] Long DA, Fleisher AJ, Douglass KO, Maxwell SE, Bielska K, Hodges JT, Plusquellic DF. Multiheterodyne spectroscopy with optical frequency combs generated from a continuous-wave laser. Opt Lett 2014;39:2688-90.

[15] Gherman T, Romanini D. Mode-locked cavity-enhanced absorption spectroscopy. Opt. Express 2002;10:1033-42.

[16] Thorpe MJ, Balslev-Clausen D, Kirchner MS, Ye J. Cavity-enhanced optical frequency comb spectroscopy: application to human breath analysis. Opt. Express 2008;16:2387-97.

[17] De Ghellinck d'Elseghem Vaernewijck X, Didriche K, Lauzin C, Rizopoulos A, Herman M, Kassi S. Cavity enhanced FTIR spectroscopy using a femto OPO absorption source. Mol Phys 2011;109:2173-9.

[18] Grilli R, Méjean G, Abd Alrahman C, Ventrillard I, Kassi S, Romanini D. Cavity-enhanced multiplexed comb spectroscopy down to the photon shot noise. Phys Rev A 2012;85:051804(R).

[19] Foltynowicz A, Masłowski P, Fleisher AJ, Bjork BJ, Ye J. Cavity-enhanced optical frequency comb spectroscopy in the mid-infrared application to trace detection of hydrogen peroxide. Appl Phys B 2013;110:163-75.

[20] Gohle C, Stein B, Schliesser A, Udem T, Hansch TW. Frequency comb vernier spectroscopy for broadband, high-resolution, high-sensitivity absorption and dispersion spectra. Phys Rev Lett 2007;99:263902.

[21] Rutkowski L, Morville J. Continuous Vernier filtering of an optical frequency comb for broadband cavity-enhanced molecular spectroscopy. J Quant Spectrosc Radiat Tranf 2017;187:204-14.

[22] Fleisher AJ, Long DA. Direct absorption spectroscopy with electro-optic frequency combs. Presentation $\mathrm{P} 2643,72^{\text {nd }}$ International Symposium on Molecular Spectroscopy, Champaign-Urbana (Illinois), june 19-23 (2017).

[23] DA Jackson. The spherical Fabry-Perot interferometer as an instrument of high resolving power for use with external or with internal atomic beams. Proc R Soc London A 1961;263:289-308.

[24] O'Keefe A, Deacon DAG. Cavity ring-down optical spectrometer for absorption measurements using pulsed laser sources. Rev Sci Instrum 1988;59:12:2544-51.

[25] Gagliardi G, Loock HP (Eds.). Cavity-enhanced spectroscopy and sensing. Springer, London, UK, (2014). 
[26] Berden G, Engelin R (Eds.). Cavity ring-down spectroscopy: techniques and applications. John Wiley \& Sons, (2009).

[27] Kogelnik H. On the propagation of Gaussian beams of light through lenslike media including those with a loss or gain variation. Appl Opt 1965;4:1562-9.

[28] Yariv A. Quantum Electronics (3rd edn). Wiley, New-York, (1989).

[29] Lehmann KK. "Dispersion and cavity-ringdown spectroscopy," Chap. 8 Cavity-Ringdown Spectroscopy, ACS Symp. Series 720, eds. KW Busch and MA Busch, 1999.

[30] Gatti D, Sala T, Gotti R, Cocola L, Poletto L, Prevedelli M, Laporta P, Marangoni M. Comb-locked cavity ring-down spectrometer. J Chem Phys 2015;142:074201.

[31] Burkart J, Sala T, Kassi S, Romanini D, Marangon M. Optical phase cloning by an integrated dualparallel Mach-Zehnder modulator. Opt Lett 2015;40:816-9.

[32] Hallal A, Bouhier S, Bodu F. Synthesis of a 30-Hz linewidth wave tunable over $500 \mathrm{GHz}$. IEEE Trans Microwave Theory and Techn 2017;65:1367-71.

[33] Galzerano G, Fasci E, Castrillo A, Colluccelli N, Gianfrani L, Laporta P. Absolute frequency stabilization of an extended-cavity diode laser against Doppler-free $\mathrm{H}_{2}{ }^{17} \mathrm{O}$ absorption lines at $1.384 \mu \mathrm{m}$. Opt Lett 2009;34:3107-9.

[34] O'Keefe A Integrated cavity output analysis of ultra-weak absorption. Chem Phys Lett 1998;293:331-6.

[35] Paul JB, Lapson L, Anderson JG. Ultrasensitive absorption spectroscopy with a high-finesse optical cavity and off-axis alignment. Appl Opt 2001;40:4904-10.

[36] Drever RWP, Hall JL, Kowalski FV, Hough J, Ford GM, Munley AJ, Ward H. Laser phase and frequency stabilization using an optical resonator. Appl Phys B 1983;31:97-105.

[37] Shaddock DA, Gray MB, McClelland DE. Frequency locking a laser to an optical cavity by use of spatial mode interference. Opt Lett 1999;24:1499-1501.

[38] Ye J, Ma LS, Hall JL. Ultrasensitive detections in atomic and molecular physics: demonstration in molecular overtone spectroscopy. J Opt Soc Am B 1998;15:6-15.

[39] Morville J, Kassi S, Chenevier M, Romanini D. Fast, low-noise, mode-by-mode, cavity-enhanced absorption spectroscopy by diode-laser self-locking. Appl Phys B 2005;80:1027-38.

[40] Kassi S, Chenevier M, Gianfrani L, Salhi A, Rouillard Y, Ouvrard A, Romanini D. Looking into the volcano with a Mid-IR DFB diode laser and Cavity Enhanced Absorption Spectroscopy. Opt Express 2006;14:11442-52.

[41] Courtillot I, Morville J, Motto-Ros V, Romanini D. Sub-ppb NO $\mathrm{N}_{2}$ detection by optical feedback cavityenhanced absorption spectroscopy with a blue diode laser. Appl Phys B 2006;85:407-12.

[42] G. Maisons, P. Gorrotxategi Carbajo, M. Carras, and D. Romanini. Optical-feedback cavity-enhanced absorption spectroscopy with a quantum cascade laser. Opt Lett 2010;35:3607-9.

[43] Richard L, Ventrillard I, Chau G, Jaulin K, Kerstel E, Romanini D. Optical-feedback cavity-enhanced absorption spectroscopy with an interband cascade laser: application to $\mathrm{SO}_{2}$ trace analysis. Appl Phys $\mathrm{B}$ 2016;122:247.

[44] Landsberg J, Romanini D, Kerstel E. Very high finesse optical-feedback cavity-enhanced absorption spectrometer for low concentration water vapor isotope analyses. Opt Lett 2014;39:1795-8.

[45] Kassi S, Campargue A. Cavity ring down spectroscopy with $5 \times 10^{-13} \mathrm{~cm}^{-1}$ sensitivity. J Chem Phys 2012;137:234201

[46] Lisak D, Hodges JT, Ciuryło R. Comparison of semiclassical line-shape models to rovibrational $\mathrm{H}_{2} \mathrm{O}$ spectra measured by frequency-stabilized cavity ring-down spectroscopy, Phys Rev A 2006;73:012507.

[47] Martínez RZ, Metsälä M, Vaittinen O, Lantta T, Halonen L. Laser-locked, high-repetition-rate cavity ringdown spectrometer. J Opt Soc Am B 2006;23:727-40.

[48] Cygan A, Lisak D, Maslowski P, Bielska K, Wojtewicz S, Domyslawska J, Trawiński RS, Ciurylo R, Abe H, Hodges JT. Pound-Drever-Hall-locked, frequency-stabilized cavity ring-down spectrometer. Rev Sci Instrum 2011;82:063107.

[49] Cygan A, Lisak D, Wójtewicz S, Domysławska J, Trawiński RS, Ciuryło R. Active control of the Pound-Drever-Hall error signal offset in high-repetition-rate cavity ring-down spectroscopy. Meas Sci Technol 2011;22:115303.

[50] Cygan A, Wójtewicz S, Domysławska J, Masłowski P, Bielska K, Piwiński M, Stec K, Trawiński RS, Ozimek F, Radzewicz C, Abe H, Ido H, Hodges JT, Lisak D, Ciuryło R. Spectral line-shapes investigation with Pound-Drever-Hall-locked frequency-stabilized cavity ring-down Spectroscopy. Eur Phys J-ST 2013;222:2119-2142.

[51] Truong GW, Douglass KO, Maxwell SE, van Zee RD, Plusquellic DF, Hodges JT, Long DA. Frequency-agile, rapid scanning spectroscopy. Nature Photonics 2013;7:532-4.

[52] Burkart J, Romanini D, Kassi S. Optical feedback stabilized laser tuned by single-sideband modulation. Opt Lett 2013;38:2062-4. 
[53] Burkart J, Sala T, Romanini D, Marangoni M, Campargue A, Kassi S. Communication: Saturated $\mathrm{CO}_{2}$ absorption near $1.6 \mu \mathrm{m}$ for kilohertz-accuracy transition frequencies. J Chem Phys 2015;142:191103.

[54] Stoltmann T, Casado M, Daëron M, Landais A, Kassi S. Direct, Precise measurements of Isotopologue Abundance Ratios in $\mathrm{CO}_{2}$ Using Molecular Absorption Spectroscopy: Application to $\Delta^{17} \mathrm{O}$. Anal Chem 2017;89:10129-32.

[55] Maddaloni P, Malara P, De Tommasi E, De Rosa M, Ricciardi I, Gagliardi G, Tamassia F, Di Lonardo G, De Natale P. Absolute measurement of the $S(0)$ and $S(1)$ lines in the electric quadrupole fundamental band of $\mathrm{D}_{2}$ around $3 \mu \mathrm{m}$. J Chem Phys 2010;133:154317.

[56] Truong GW, Long DA, Cygan A, Lisak D, van Zee RD, Hodges JT. Comb-linked, cavity ring-down spectroscopy measurements of molecular transition frequencies at the kHz-level. J Chem Phys 2013;138:094201.

[57] Mondelain D, Sala T, Kassi S, Romanini D, Marangoni M, Campargue A. Broadband and highly sensitive comb-assisted cavity ring down spectroscopy of CO near $1.57 \mu \mathrm{m}$ with sub-MHz frequency accuracy. J Quant Spectrosc Radiat Transf 2015;154:35-43.

[58] Mondelain D, Mikhailenko SN, Karlovets EV, Béguier S, Kassi S, Campargue A. Comb-Assisted Cavity Ring Down Spectroscopy of ${ }^{17} \mathrm{O}$ enriched water between 7443 and $7921 \mathrm{~cm}^{-1}$. J Quant Spectrosc Radiat Transf 2017;203:206-12.

[59] Mikhailenko SN, Mondelain D, Karlovets EV, Kassi S, Campargue A. Comb-Assisted Cavity Ring Down Spectroscopy of ${ }^{17} \mathrm{O}$ enriched water between 6667 and $7443 \mathrm{~cm}^{-1}$. J Quant Spectrosc Radiat Transf 2018;206:163-71.

[60] Odintsova TA, Fasci E, Moretti L, Zak EJ, Polyansky OL, Tennyson J, Gianfrani L, Castrillo A. Highly accurate intensity factors of pure $\mathrm{CO}_{2}$ lines near $2 \mu \mathrm{m}$. J Chem Phys 2017; 146:244309.

[61] Yamada KMT, Onae A, Hong F-L, Inaba H, Matsumoto H, Nakajima Y, Ito F, Shimizu T. High precision line profile measurements on ${ }^{13} \mathrm{C}$ acetylene using near infrared frequency comb spectrometer. J Mol Spectrosc. 2008;249:95-99.

[62] Yamada KMT, Onae A, Hong FL, Inaba H, Shimizu T. Precise determination of the Doppler width of a rovibrational absorption line using a comb-locked diode laser. C R Physique 2009;10:907-15.

[63] Sala T, Kassi S, Burkart J, Marangoni M, Romanini D. Comb-assisted coherence transfer between laser fields. arXiv:1412.1102 [physics.optics].

[64] Cygan A, Lisak D, Morzyński P, Bober M, Zawada M, Pazderski E, Ciuryło R. Cavity mode-width spectroscopy with widely tunable ultra narrow laser. Opt Express 2013;21:29744-54.

[65] Long DA, Truong GW, van Zee RD, Plusquellic DF, Hodges JT. Frequency-agile, rapid scanning spectroscopy: Absorption sensitivity of $2 \times 10^{-12} \mathrm{~cm}^{-1} \mathrm{~Hz}^{-1 / 2}$ with a tunable diode laser. Appl Phys B 2014;114:489-95.

[66] Cygan A, Wcisło P, Wójtewicz S, Masłowski P, Trawinski RS, Ciuryło R, Lisak D. Alternative approaches to cavity enhanced absorption spectroscopy. J Phys Conf Ser 2014;548:012024.

[67] Cygan A, Wójtewicz S, Kowzan G, Zaborowski M, Wcisło P, Nawrocki J, Krehlik P, Śliwczyński Ł, Lipiński M, Masłowski P, Ciuryło R, Lisak D. Absolute molecular transitions frequencies measured by three cavity-enhanced spectroscopy techniques. J Chem Phys 2016;144:214202.

[68] Cygan A, Wójtewicz S, Zaborowski M, Wcisło P, Guo R, Ciuryło R, Lisak D. One-dimensional cavity mode-dispersion spectroscopy for validation of CRDS technique. Meas Sci Technol 2016;27:045501.

[69] Tan S, Berceau P, Saraf S, Lipa JA. Measuring finesse and gas absorption with Lorentzian recovery spectroscopy. Opt Express 2017;25:7645-56.

[70] Rutkowski L, Johansson AC, Zhao G, Hausmaninger T, Khodabakhsh A, Axner O, Foltynowicz A. Sensitive and broadband measurement of dispersion in a cavity using a Fourier transform spectrometer with $\mathrm{kHz}$ resolution. Opt Ecpress 2017;25:21711-8.

[71] Charczun D, Kowzan G, Cygan A, Kropidłowska K, Zawitowska J, Trawiński RS, Lisak D, Maslowski $\mathrm{P}$. Fourier transform spectrometry with $\mathrm{kHz}-\mathrm{level}$ resolution for broadband cavity mode-width spectroscopy. Cavity Enhanced Spectroscopy 2017, 12th International User Meeting, Egmont aan Zee, Netherlands.

[72] Masłowski P, Lee KF, Johansson AC, Khodabakhsh A, Kowzan G, Rutkowski L, Mills AA, Mohr C, Jiang J, Fermann ME, Foltynowicz A. Surpassing the path-limited resolution of Fourier-transform spectrometry with frequency combs. Phys Rev A 2016;93:021802(R).

[73] Lisak D, Cygan A, Wójtewicz S, Wcisło P, Zaborowski M, Kowzan G, Masłowski P, Ciuryło R. Spectral line-shape study by cavity-enhanced complex refractive index spectroscopy. J Phys Conf Ser 2017;810:012007.

[74] Foltynowicz A, Masłowski P, Ban T, Adler F, Cossel KC, Briles TC, Ye J. Optical frequency comb spectroscopy. Faraday Discuss 2011;150:23-31.

[75] Schiller S. Spectrometry with frequency combs. Opt Lett 2002;27:766-8. 
[76] Keilmann F, Gohle C, Holzwarth R. Time-domain mid-infrared frequeny-comb spectrometer. Opt Lett 2004;29:1542-4.

[77] Schliesser A, Brehm M, Keilmann F, van der Weide DW. Frequency-comb infrared spectrometer for rapid, remote chemical sensing. Opt Express 2005;13:9029-38.

[78] Brehm M, Schliesser A, Keilmann F. Spectroscopic near-field microscopy using frequency combs in the mid-infrared. Opt Express 2006;14:11222-33.

[79] Coddington I, Swann WC, Newbury NR. Newbury. Coherent Multiheterodyne Spectroscopy Using Stabilized Optical Frequency Combs. Phys Rev Lett 2008;100:013902.

[80] Chamberlain J. The Principles of Interferometric Spectroscopy. John Wiley and Sons, New York, (1979).

[81] Coddington I, Swann WC, Newbury NR. Coherent multiheterodyne spectroscopy using stabilized optical frequency combs. Phys Rev Lett 2008;100:013902.

[82] Rieker GB, Giorgetta FR, Swann WC, Kofler J, Zolot AM, Sinclair LC, Baumann E, Cromer C, Petron G, Sweeney C, Tans PP, Coddington I, Newbury NR. Frequency-comb-based remote sensing of greenhouse gases over kilometer air paths. Optica 2014;1:290-8.

[83] Baumann E, Giorgetta FR, Swann WC, Zolot AM, Coddington I, Newbury NR. Spectroscopy of the methane $v_{3}$ band with an accurate midinfrared coherent dual-comb spectrometer. Phys Rev A 2011;84:062513.

[84] Fleisher AJ, Long DA, Reed ZD, Hodges JT, Plusquellic DF. Coherent cavity-enhanced dual-comb spectroscopy. Opt Express 2016;24:10424-34.

[85] Rutkowski L, Masłowski P, Johansson AC, Khodabakhsh A, Foltynowicz A. Optical frequency comb Fourier transform spectroscopy with sub-nominal resolution. arXiv:1612.04808 (2017).

[86] Kowzan G, Lee KF, Paradowska M, Borkowski M, Ablewski P, Wójtewicz S, Stec K, Lisak D, Fermann ME, Trawiński RS, Masłowski P. Self-referenced, accurate and sensitive optical frequency comb spectroscopy with VIPA spectrometer. Opt Lett 2016;41:974-7.

[87] Foltynowicz A, Ban T, Masłowski P, Adler F, Ye J. Quantum noise-limited optical frequency comb spectroscopy. Phys Rev Lett 2011;107:233002.

[88] Diddams SA, Hollberg L, Mbele V. Molecular fingerprinting with the resolved modes of a femtosecond laser frequency comb. Nature 2007;445:627-30.

[89] Scholten SK, Anstie JD, Hébert NB, White RT, Genest J, Luiten AN. Complex direct comb spectroscopy with a virtually imaged phased array. Opt Lett 2016;41:1277-80.

[90] Kowzan G, Charczun D, Cygan A, Trawiński RS, Lisak D, Masłowski P. Broadband VIPA spectrometer with sub-kHz resolution for cavity mode-width spectroscopy. Quantum Optics IX, 2017, Gdańsk, Poland.

[91] Castrillo A, Fasci E, Galzerano G, Casa G, Laporta P, Gianfrani L. Offset frequency locking of extended-cavity diode lasers for precision spectroscopy of water at $1.38 \mu \mathrm{m}$. Opt Express 2010;18:21851-60.

[92] Gagliardi G, Rusciano G, and Gianfrani L. Narrow $\mathrm{H}_{2}{ }^{18} \mathrm{O}$ lines and new absolute frequency references in the near-IR. J Opt A 2000;2:310-3.

[93] De Vizia MD, Rohart F, Castillo A, Fasci E, Moretti L, Gianfrani L. Speed-dependent effects in the near-infrared spectrum of self-colliding $\mathrm{H}_{2}{ }^{18} \mathrm{O}$ molecules. Phys Rev A 2011;83:052506.

[94] De Vizia MD, Castrillø A, Fasci E, Moretti L, Rohart F, and Gianfrani L. Speed dependence of collision parameters in the $\mathrm{H}_{2}{ }^{18} \mathrm{O}$ near-IR spectrum: Experimental test of the quadratic approximation. Phys Rev A 2012,85:062512.

[95] Dinesan H, Fasci E, Castrillo A and Gianfrani L. Absolute frequency stabilization of an extended-cavity diode laser by means of noise-immune cavity enhanced optical heterodyne molecular spectroscopy. Opt Lett 2014;39:2198-201.

[96] Fasci E, Odintsova TA, Castrillo A, De Vizia MD, Merlone A, Bertiglia F, Moretti L, Gianfrani L. Dual-laser absorption spectroscopy of $\mathrm{C}_{2} \mathrm{H}_{2}$ at $1.4 \mu \mathrm{m}$. Phys Rev A 2016;93:042513.

[97] Matthey R, Schilt S, Werner D, Affolderbach C, Thévenaz L, Mileti G. Diode laser frequency stabilisation for water-vapour differential absorption sensing. Appl Phys B 2006;85:477-85.

[98] Schilt S, Matthey R, Kauffman-Werner D, Affolderbach C, Mileti G, Thévenaz L. Laser offsetfrequency locking up to $20 \mathrm{GHz}$ using a low-frequency electrical filter technique. Appl Opt 2008;47:4336-44.

[99] Benner DC, Rinsland CP, Devi VM, Smith MAH, Atkins D. A multispectrum nonlinear least squares fitting technique. J Quant Spectrosc Radiat Transf 1995;53:705-21.

[100] Drouin BJ, Benner DC, Brown LR, Cich MJ, Crawford TJ, Malathy Devi V, Guillaume A, Hodges JT, Mlawer EJ, Robichaud DJ, Oyafuso F, Payne VH, Sung K, Wishnow EW, Yu S. Multispectrum analysis of the oxygen A-band. J Quant Spectrosc Radiat Transf 2017;186:118-38. 
[101] Mantz AW, Sung K, Brown LR, Crawford TJ, Smith MAH, Devi VM, Benner DC. A cryogenic Herriott cell vacuum-coupled to a Bruker IFS-125HR. J Mol Spectrosc 2014;304:12-24.

[102] Devi VM, Benner DC, Sung K, Crawford TJ, Yu S, Brown LR, Smith MAH, Mantz AW, Boudon V, Ismail S. Self- and air-broadened line shapes in the $2 v_{3} \mathrm{P}$ and $\mathrm{R}$ branches of ${ }^{12} \mathrm{CH}_{4}$. J Mol Spectros 2015;315:114-36.

[103] Wilzewski JS, Birk M, Loos J, Wagner G. Temperature-dependence laws of absorption line shape parameters of the $\mathrm{CO}_{2} v_{3}$ band. JQSRT 2018;206:296-305.

[104] Hubers HW, Kimmitt MF, Hiromoto N, Brundermann E. Terahertz spectroscopy: System and sensitivity considerations, IEEE Trans Terahertz Sci Tech 2001;1;321-31.

[105] Hubers HW, Pavlov SG, Richter H, Semenov AD. High-resolution gas phase spectroscopy with a distributed feedback terahertz quantum cascade laser. Appl Phys Lett 2006;89:061115.

[106] Vitiello MS, Consolino L, Bartalini S, Taschin A, Tredicucci A, Inguscio M, De Natale P. Quantumlimited frequency fluctuations in a terahertz laser. Nature Photonics 2012;6:525-8.

[107] Hubers HW, Eichholz R, Pavlov SG, Richter H. High resolution terahertz spectroscopy with quantum cascade lasers. J Infrar Milli Terahz Waves 2013;34:325-41.

[108] Bartalini S, Consolino L, Cancio P, De Natale P, Bartolini P, Taschin A, De Pas M, Beere H, Ritchie D, Vitiello MS, Torre R. Frequency-comb-assisted terahertz quantum cascade laser spectroscopy. Phys Rev X 2014;4:021006.

[109] Podobedov VB, Plusquellic DF, Fraser GT. THz laser study of self-pressure and temperature broadening and shifts of water vapor lines for pressures up to $1.4 \mathrm{kPa}$, J Quant Spectrosc Radiat Transf 2004;87:377-85.

[110] Cazzoli G, Dore L, Puzzarini C, Bakri B, Colmont JM, RohartF, Wlodarczak G. Experimental determination of air-broadening parameters of pure rotational transitions of $\mathrm{HNO}_{3}$ : intercomparison of measurements by using different techniques. J Mol Spectrosc 2005;229:158-69.

[111] Cazzoli G, Puzzarini C, Buffa G, Tarrini O. Pressure-broadening in the THz frequency region: The $1.113 \mathrm{THz}$ line of water, J Quant Spectrosc Radiat Transf 2008;109:1563-74.

[112] Cazzoli G, Puzzarini C, Buffa G, Tarrini O. Pressure-broadéning of water lines in the THz frequency region: Improvements and confirmations for spectroscopic databases. Part II. J Quant Spectrosc Radiat Transf 2009;110:609-18.

[113] Slocum DM, Giles RH, Goyette TM. High-resolution water vapor spectrum and line shape analysis in the Terahertz region. J Quant Spectrosc Radiat Transf 2015;159:69-79.

[114] Gopalsami N, Raptis AC, Meier J. Millimeter-wave cavity ringdown spectroscopy. Rev Sci Instrum 2002;73:259-62.

[115] Meshkov AI, De Lucia FC. Broadband absolute absorption measurements of atmospheric continua with millimeter wave cavity ringdown spectroscopy. Rev Sci Instrum 2005;76:083103.

[116] Drouin BJ, Tang A, Schlecht E, Brageot E, Gu OJ, Ye Y, Shu R, Chang MF, Kim Y. A CMOS millimeter-wave transceiver embedded in a semi-confocal Fabry-Perot cavity for molecular spectroscopy. J Chem Phys 2016;145:074201.

[117] Hartmann JM, Tran H, Ngo NH, Landsheere X, Chelin P, Lu X, Liu AW, Hu SM, Gianfrani L, Casa G, Castrillo A, Lepère M, Delière Q, Dhyne M, Fissiaux L. Ab initio calculations of the spectral shapes of $\mathrm{CO}_{2}$ isolated lines including non-Voigt effects and comparisons with experiments. Phys Rev A 2013;87:013403.

[118] Larcher G, Tran H, Schwell M, Chelin P, Landsheere X, Hartmann JM, Hu SM. CO 2 isolated line shapes by classical molecular dynamics simulations: Influence of the intermolecular potential and comparison with new measurements. J Chem Phys 2014;140:084308.

[119] Hartmann JM, Sironneau V, Boulet C, Svensson T, Hodges JT, Xu CT. Collisional broadening and spectral shapes of absorption lines of free and nanopore-confined $\mathrm{O}_{2}$ gas. Phys Rev A 2013;87:032510.

[120] Lamouroux J, Sironneau V, Hodges J, Hartmann JM. Isolated line shapes of molecular oxygen: Classical molecular dynamics versus measurements. Phys Rev A 2014;89:042504.

[121] Tran H, Domenech JL. Spectral shapes of Ar-broadened HCl lines in the fundamental band by classical molecular dynamics simulations and comparison with experiments. J Chem Phys 2014;141:064313.

[122] Le T, Domenech JL, Lepère $\mathrm{M}$, Tran H. Molecular dynamic simulations of $\mathrm{N}_{2}$-broadened methane line shapes and comparison with experiments. J Chem Phys 2017;146:094305.

[123] Lamouroux J, Hartmann JM, Tran H, Lavorel B, Snels M, Stefani S, Piccioni G. Molecular dynamics simulations for $\mathrm{CO}_{2}$ spectra. IV. Collisional line-mixing in infrared and Raman bands. J Chem Phys 2013;138:244310.

[124] Tran H, Li G, Ebert V, Hartmann JM. Super- and sub-Lorentzian effects in the Ar-broadened line wings of $\mathrm{HCl}$ gas. J Chem Phys 2017;146:194305. 
[125] Ngo NH, Hartmann JM. A strategy to complete databases with parameters of refined line shapes and its test for CO in He, Ar and Kr. J Quant Spectrosc Radiat Transf 2017;203:334-40.

[126] Rautian SG, Sobel'man II. The effect of collisions on the Doppler broadening of spectral lines. Soviet Physics Uspekhi 1967;9:701-16.

[127] Smith EW, Cooper J, Chappell WR, Dillon T. An impact theory for Doppler and pressure broadening -I General theory. J Quant Spectrosc Radiat Transf 1971;11:1547-65.

[128] Smith EW, Cooper J, Chappell WR, Dillon T. An impact theory for Doppler and pressure broadening II Atomic and molecular systems. J Quant Spectrosc Radiat Transf 1971;11:1567-76.

[129] Ciuryło R, Pine AS. Speed-dependent line mixing profiles. J Quant Spectrosc Radiat Transf 2000;67:375-93.

[130] May AD, Liu WK, McCourt FRW, Ciuryło R, Sanchez-Fortun Stoker J, Shapiro D, Wehr R. The impact theory of spectral line shapes: a paradigm shift. Can J Phys 2013;91:879-95.

[131] Wcisło P, Ciuryło R. Influence of the interaction potential shape on the Dicke narrowed spectral line profiles affected by speed-dependent collisional broadening and shifting. J Quant Spectrosc Radiat Transfer 2013;120:36-43.

[132] Keilson J, Storer JE. On Brownian motion, Boltzmann's equation, and the Fokker-Planck equation. Quart Appl Math 1952;10:243-53.

[133] Robert D, Bonamy L. Memory effects in speed-changing collisions and their consequences for spectral lineshape: I. Collision regime. Eur Phys J D 1998;2:245-52.

[134] Nelkin M, Ghatak A. Simple binary collision model for Van Hove's G $\mathrm{s}_{\mathrm{s}}$,rt). Phys Rev 1964;135:A4-9.

[135] Galatry L. Simultaneous effect of Doppler and foreign gas broadening on spectral lines. Phys Rev $1961 ; 122: 1218-23$.

[136] Bonamy L, Tran H, Joubert P, Robert D. Memory effects in speed-changing collisions and their consequences for spectral line shape. Eur Phys J D 2004;31:459-67.

[137] Joubert P, Hoang PNM, Bonamy L, Robert D. Speed-dependent line-shape model analysis from molecular-dynamics simulations: The collisional confinement narrowing regime. Phys Rev A 2002;66:042508.

[138] Tran H, Joubert P, Bonamy L, Lavorel B, Renard V, Chaussard F, Faucher O, Sinardet B. Femtosecond time resolved coherent anti-Stokes Raman spectroscopy: Experiment and modelization of speed memory effects on $\mathrm{H}_{2}-\mathrm{N}_{2}$ mixtures in the collision regime. J Chem Phys 2005;122:194317.

[139] Tran H, Chaussard F, Le Cong N, Lavorel B, Faucher O, Joubert P. Femtosecond time resolved coherent anti-Stokes Raman spectroscopy of $\mathrm{H}_{2}-\mathrm{N}_{2}$ mixtures in the Dicke regime: Experiments and modeling of velocity effects. J Chem Phys 2009;131:174310.

[140] Ngo NH, Tran H, Gamache RR. A pure $\mathrm{H}_{2} \mathrm{O}$ isolated line-shape model based on classical molecular dynamics simulations of velocity changes and semi-classical calculations of speed-dependent collisional parameters. J Chem Phys 2012;136:154310.

[141] Ngo NH, Tran H, Gamache RR, Bermejo D, Domenech JL. Influence of velocity effects on the shape of $\mathrm{N}_{2}$ (and air) broadened $\mathrm{H}_{2} \mathrm{O}$ lines revisited with classical molecular dynamics simulations. J Chem Phys 2012;137:064302.

[142] Tran H, Ngo NH,Hartmann JM, Gamache RR, Mondelain D, Kassi S, Campargue A, Gianfrani L, Castrillo A, Fasci E, Rohart F. Velocity effects on the shape of pure $\mathrm{H}_{2} \mathrm{O}$ isolated lines: Complementary tests of the partially correlated speed-dependent Keilson-Storer model. J Chem Phys 2013;138:034302.

[143] Ngo NH, Tran H. Precise predictions of $\mathrm{H}_{2} \mathrm{O}$ line shapes over a wide pressure range using simulations corrected by a single measurement. J Quant Spectrosc Radiat Transf 2018;207:16-22.

[144] Tran Ha, Hartmann JM, Chaussard F, Gupta M. An isolated line-shape model based on the KeilsonStorer function for velocity changes. II. Molecular Dynamics Simulations and the Q(1) lines for pure $\mathrm{H}_{2}$. J Chem Phys 2009; 131:154303.

[145] Tran Ha, Hartmann JM. An isolated line-shape model based on the Keilson and Storer function for velocity changes. I. Theoretical approaches. J Chem Phys 2009;130:094301.

[146] Lindenfeld MJ, Shizgal B. Matrix elements of the Boltzmann collision operator for gas mixtures. Chem Phys 1979;41:81-95.

[147] Lindenfeld MJ. Self-structure factor of hard-sphere gases for arbitrary ratio of bath to test particle masses. J Chem Phys1980;73:5817-29.

[148] Liao PF, Bjorkholm JE, Berman PR. Effects of velocity-changing collisions on two-photon and stepwise-absorbtion spectroscopic line shapes. Phys Rev A 1980;21:1927-38.

[149] Wcisło P, Cygan A, Lisak D, Ciuryło R. Iterative approach to line-shape calculations based on the transport-relaxation equation. Phys Rev A 2013;88:012517.

[150] Ciuryło R, Shapiro DA, Drummond JR, May AD. Solving the line-shape problem with speed-dependent broadening and shifting and with Dicke narrowing. II. Application. Phys Rev A 2002;65:012502-8. 
[151] Wcisło P, Lisak D, Ciuryło R, Pine AS. Multispectrum-fitting of phenomenological collisional lineshape models to a speed-dependent Blackmore profile for spectroscopic analysis and databases. J Phys Conf Ser 2017;810:012061.

[152] Wcisło P, Gordon IE, Cheng CF, Hu SM, Ciuryło R. Collision-induced line-shape effects limiting the accuracy in Doppler-limited spectroscopy of $\mathrm{H}_{2}$. Phys Rev A 2016;93:022501.

[153] Wcisło P, Gordon IE, Tran H, Tan Y, Hu SM, Campargue A, Kassi S, Romanini D, Hill C, Kochanov $\mathrm{RV}$, Rothman LS. The implementation of non-Voigt line profiles in the HITRAN database: $\mathrm{H}_{2}$ case study. J Quant Spectrosc Radiat Transf 2016;177:75-91.

[154] Tran H, Thibault F, Hartmann JM. Collision-induced velocity changes from molecular dynamic simulations in $\mathrm{H}_{2}$-Ar: A test of the Keilson-Storer model and of line-broadening/shifting calculations for the Q(1) Raman line. J Quant Spectrosc Radiat Transf 2011;112:1035-42.

[155] Wcisło P, Thibault F, Cybulski H, Ciuryło R. Strong competition between velocity-changing and phaseot state-changing collisions in $\mathrm{H}_{2}$ spectra perturbed by Ar. Phys Rev A 2015;91:052505.

[156] Thibault F, Wcislo P, Ciurylo R. A test of $\mathrm{H}_{2}$-He potential energy surface. Eur Phys J D 2016;70:236.

[157] Berger JP, Saint-Loup R, Berger H, Bonamy J, Robert D. Measurement of vibrational line profiles in $\mathrm{H}_{2}$-rare-gas mixtures: Determination of the speed dependence of the line shift. Phys Rev A 1994;49:3396-406.

[158] Chaussard F, Michaut X, Saint-Loup R, Berger H, Joubert P, Lance B, Bonamy J, Robert D. Collisional effects on spectral line shape from the Doppler to the collisional regime: A rigorous test of a unified model. J Chem Phys 2000;112:158-66.

[159] Wcisło P, Tran H, Kassi S, Campargue A, Thibault F, Ciuryło R. Velocity-changing collisions in pure $\mathrm{H}_{2}$ and $\mathrm{H}_{2}$-Ar mixture. J Chem Phys 2014;141:074301.

[160] Huang YS, Chiue JH, Huang YC, Hsiung TC. Relativistic formulation for the Doppler-broadened line profile. Phys Rev A 2010;82:010102(R).

[161] Wcisło P, Amodio P, Ciuryło R, Gianfrani L. Relativistic formulation of the Voigt profile. Phys Rev A 2015;91:022508.

[162] Gianfrani L. Linking the thermodynamic temperature to an optical frequency: recent advances in Doppler broadening thermometry. Phil Trans R Soc A 2016;374:20150047.

[163] Wójtewicz S, Wcisło P, Lisak D, Ciuryło R. Dispersion corrections to the Gaussian profile describing the Doppler broadening of spectral lines. Phys Rev A 2016;93:042512.

[164] Rohart F, Mäder H, Nicolaisen HW. Speed dependence of rotational relaxation induced by foreign gas collisions: Studies on $\mathrm{CH}_{3} \mathrm{~F}$ by millimeter wave coherent trasients. J Chem Phys 1994;101:6475-86.

[165] Rofart D, Ellendt A, Kaghat F, Mäder H. Self and polar foreign gas line broadening and frequency shifting of $\mathrm{CH}_{3} \mathrm{~F}$ : Effect of the speed dependence observed by millimeter-wave coherent transients. J Mol Spectrosc 1997; 185:222-33.

[166] Berman PR. Speed-dependent collisional width and shift parameters in spectral profiles. J Quant Spectrosc Radiat Transf 1972;12:1331-42.

[167] Pickett HM. Effects of velocity averaging of the shapes of absorption lines. J Chem Phys 1980;73:60904.

[168] Ngo NH, Lin H, Hodges JT, Tran H. Spectral shapes of rovibrational lines of CO broadened by He, Ar, $\mathrm{Kr}$ and $\mathrm{SF}_{6} . \mathrm{J}$ Quant Spectrosc Radiat Transf 2017;203:325-33.

[169] Ngo NH, Lisak D, Tran H, Hartmann JM. An isolated line-shape model to go beyond the Voigt profile in spectroscopic databases and radiative transfer codes. J Quant Spectrosc Radiat Transf 2013;129:89100. Associated Erratum: J Quant Spectrosc Radiat Transf 2014;134:105.

[170] Cygan A, Lisak D. Multi-spectrum fitting software for advanced spectral line shapes analysis. J Phys Conf Ser 2017;810:012025.

[171] Pine AS. Asymetries and correlations in speed-dependent Dicke-narrowed line shapes of argonbroadened HF. J Quant Spectrosc Radiat Tranf 1999;62:397-423.

[172] Humlicek J. Optimized computation of the Voigt and complex probability functions. J Quant Spectrosc Radiat Transf 1982;27:437-44.

[173] Kuntz M. A new implementation of the Humlicek algorithm for the calculation of the Voigt profile function. J Quant Spectrosc Radiat Transf 1997;57:819-24.

[174] Wells RJ. Rapid approximation to the Voigt/Faddeeva function and its derivatives. J Quant Spectrosc Radiat Transf 1999;62:29-48.

[175] Letchworth KL, Benner DC. Rapid and accurate calculation of the Voigt function. J Quant Spectrosc Radiat Tranfer 2007;107:173-92.

[176] Schreider F. Optimized implementations of rational approximations for the Voigt and complex error function. J Quant Spectrosc Radiat Transf 2011;112:1010-25.

[177] Boone CD, Walker KA, Bernath PF. An efficient analytical approach for calculating line mixing in atmospheric remote sensing applications. J Quant Spectrosc Radiat Transf 2011;112:980-9. 
[178] Tran H, Ngo NH, Hartmann JM. Efficient computation of some speed-dependent isolated line profiles. J Quant Spectrosc Radiat Transf 2013;129:199-203. Associated Erratum: J Quant Spectrosc Radiat Transf 2014;134:104.

[179] Tennyson J, Bernath PF, Campargue A, Csaszar AG, Daumont L, Gamache RR, Hodges JT, Lisak D, Naumenko OV, Rothman LS, Tran H, Zobov NF, Buldyreva J, Boone DC, Hartmann JM, McPheat R, Weidmann D, Murray J, Ngo NH, Polyansky OL. Recommended isolated-line profile for representing high-resolution spectroscopic transitions. Pure Appl Chem 2014;86:1931-43.

[180] Lisak D, Cygan A, Wcisło P, Ciuryło R. Quadratic speed dependence of collisional broadening and shifting for atmospheric applications. J Quant Spectrosc Radiat Transf 2015;151:43-8.

[181] Ghysels M, Liu Q, Fleisher AJ, Hodges JT. A variable-temperature cavity ring-down spectrometer with application to line shape analysis of $\mathrm{CO}_{2}$ spectra in the $1600 \mathrm{~nm}$ region. Appl Phys B 2017;123:124.

[182] Goldenstein CS, Hanson RK. Diode-laser measurements of line strength and temperature-dependent line shape parameters for $\mathrm{H}_{2} \mathrm{O}$ transitions near $1.4 \mu \mathrm{m}$ using Voigt, Rautian, Galatry, and speeddependent Voigt profiles. J Quant Spectrosc Radiat Transf 2015;152:127-39.

[183] Cich MJ, Forthomme D, McRaven CP, Lopez GV, Hall GE, Sears TJ, Mantz AW. Temperaturedependent, nitrogen-perturbed line shape measurements in the $v_{1}+v_{3}$ band of acetylene using a diode laser referenced to a frequency comb. J Phys Chem A 2013;117:13908-18.

[184] Forthomme D, Cich MJ, Twagirayezu S, Hall GE, Sears TJ. Application of the Hartmann-Tran profile to precise experimental data sets of ${ }^{12} \mathrm{C}_{2} \mathrm{H}_{2}$. J Quant Spectrosc Radiat Transf 2015;165:28-37.

[185] Li H, Farooq A, Jeffries JB, Hanson RK. Diode laser measurements of temperature-dependent collisional-narrowing and broadening parameters of Ar-perturbed $\mathrm{H}_{2} \mathrm{O}$ transitions at 1391.7 and 1397.8 nm. J Quant Spectrosc Radiat Transf 2008;109:132-43.

[186] Goldenstein CS, Jeffries JB, Hanson RK. Diode laser measurements of linestrength and temperaturedependent lineshape parameters of $\mathrm{H}_{2} \mathrm{O}-, \mathrm{CO}_{2^{-}}$, and $\mathrm{N}_{2}$-perturbed $\mathrm{H}_{2} \mathrm{O}$ transitions near 2474 and 2482 nm. J Quant Spectrosc Radiat Transf 2013;130:100-11.

[187] Domysławska J, Wójtewicz S, Cygan A, Bielska K, Lisak D, Masłowski P, Trawinski RS, Ciuryło R. Low-pressure line-shape study in molecular oxygen with absolute frequency reference. J Chem Phys 2013;139:194312.

[188] Lisak D, CyganA, Bermejo D, Domenech JL, Hodges JT, Tran H. Application of the Hartmann-Tran profile to analysis of $\mathrm{H}_{2} \mathrm{O}$ spectra. J Quant Spectrosc Radiat Transf 2015;164:221-30.

[189] De Vizia MD, Castrillo A, Fasci E, Amodio P, Moretti L, Gianfrani L. Experimental test of the quadratic approximation in the partially correlated speed-dependent hard-collision profile. Phys Rev A 2014;90:022503.

[190] Kochanov VP. Speed-dependent spectral line profile including line narrowing and mixing. J Quant Spectrosc Radiat Transf 2016;177:261-8.

[191] Hutson JM, Green S. MOLSCAT computer code, version 14 (1994) distributed by Collaborative Computational Project No. 6 of the Engineering and Physical Sciences Research Council (UK). https://www.giss.nasa.gov/tools/molscat/. PMP MOLSCAT, developed by G.C. McBane and P. Valiron, is available at http://faculty.gvsu.edu/mcbaneg/pmpmolscat.

[192] Kochanov VP. Combined effect of small- and large-angle scattering collisions on a spectral line shape. J Quant Spectrosc Radiat Transf 2015;159:32-8.

[193] Thibault F, Ivanov SV, Buzykin OG, Gomez L, Dhyne M, Joubert P, Lepère M. Comparison of classical, semiclassical and quantum methods in hydrogen broadening of acetylene lines. J Quant Spectrosc Radiat Transf 2011;112:1429-37.

[194] Gomez L, Ivanov SV, Buzykin OG, Thibault F. Comparison of quantum, semiclassical and classical methods in hydrogen broadening of nitrogen lines. J Quant Spectrosc Radiat Transf 2011;112:1942-9.

[195] ThibaultF, Gomez L, Ivanov SV, Buzykin OG, Boulet C. Comparison of quantum, semi-classical and classical methods in the calculation of nitrogen self-broadened widths. J Quant Spectrosc Radiat Transf 2012;113:1887-97.

[196] Ivanov SV, Buzykin OG. Pressure broadening of the electric dipole and Raman lines of $\mathrm{CO}_{2}$ by Argon: Stringent test of the classical theory at different temperatures on a benchmark system. J Quant Spectrosc Radiat Transf 2016;185:48-57.

[197] Bernstein RB editor. Atom-molecule theory: A Guide for the Experimentalist. Plenum Press, New York, (1979).

[198] Flower DR, Bourhis G, Launay JM. Molcol: A program for solving atomic and molecular collision problems. Comput Phys Comm 2000;131:187-201.

[199] http://www2.chem.umd.edu/groups/alexander/hibridon/hib43/.

[200] Dubernet ML, Tuckey PA. Raman Q and S line broadening and shifting coefficients: some commonly used assumptions revisited. Chem Phys Lett 1999;300:275-80. 
[201] Grimminck DLAG, Spiering FR, Janssen LMC, van der Avoird A, van der Zande WJ, Groenenboom GC. A theoretical and experimental study of pressure broadening of the oxygen A-band by Helium. J Chem Phys 2014;140:204314.

[202] Dagdigian PJ. Pressure broadening calculations for OH in collisions with argon: Rotational, vibrational, and electronic transitions. J Quant Spectrosc Radiat Transf 2017;189:105-11.

[203] Wiesenfeld L, Faure A. Ab initio computation of the broadening of water rotational lines by molecular hydrogen. Phys Rev A 2010;82:040702.

[204] Drouin B, Wiesenfeld L. Low-temperature water-hydrogen-molecule collisions probed by pressure broadening and line shift. Phys Rev A 2012;86:022705.

[205] Faure A, Wiesenfeld L, Drouin BJ, Tennyson J. Pressure broadening of water and carbon monoxide transitions by molecular hydrogen at high temperatures. J Quant Spectrosc Radiat Transf 2013;116:7986.

[206] Wiesenfeld L, Faure A. Rotational quenching of $\mathrm{H}_{2} \mathrm{CO}$ by molecular hydrogen: Cross-sections, rates and pressure broadening. MNRAS 2013;432:2573-8.

[207] Thibault F, Patkowski K, Zuchowski P, Jozwiak H, Ciurylo R, Wcislo P. Rovibrational line-shape parameters for $\mathrm{H}_{2}$ in $\mathrm{He}$ from and new $\mathrm{H}_{2}-\mathrm{He}$ potential energy surface. J Quant Spectrosc Radiat Transf 2017;202:308-20.

[208] Hess S. Kinetic theory of spectral line shapes. The transition between Doppler broadening and collisional broadening. Physica 1972;61:80-94.

[209] Monchick L, Hunter LW. Diatomic-diatomic molecular collision integrals for pressure broadening and Dicke narrowing: A generalization of Hess's theory. J Chem Phys 1986;85:713-8.

[210] Thibault F, Cappelletti D, Pirani F, Blanquet G, Bartolomei M. Molecular-beam scattering and pressure broadening cross sections for the acetylene-neon system. Eur Phys J D. 2007;44:337-44.

[211] Thibault F, Fuller EP, Grabow KA, Hardwick JL, Marcus CI, Marston D, Robertson LA, Senning EN, Stoffel MC, Wiser RS. Experimental line broadening and line shift coefficients of the acetylene $v_{1}+v_{3}$ band pressurized by hydrogen and deuterium and comparison with calculations. J Mol Spectrosc 2009;256:17-27.

[212] Deng W, Mondelain D, Thibault F, Camy-Peyret C, Mantz AW. Experimental He-pressure broadening for the $\mathrm{R}(10)$ and $\mathrm{P}(2)$ lines in the $\mathrm{v}_{3}$ band of ${ }^{13} \mathrm{CO}_{2}$, and experimental pressure shifts for $\mathrm{R}(10)$ measured at several temperatures between $300 \mathrm{~K}$ and $100 \mathrm{~K}$. J Mol Spectrosc 2009;256:102-8.

[213] Ronningen TJ, De Lucia FC. Helium induced pressure broadening and shifting of HCN hyperfine transitions between 1.3 and $20 \mathrm{~K}$. J Chem Phys 2005;122:184319.

[214] Thibault F, Corretja B, Viel A, Bermejo D, Martinez RZ, Bussery-Honvault B. Linewidths of $\mathrm{C}_{2} \mathrm{H}_{2}$ perturbed by $\mathrm{H}_{2}$ : Experiments and calculations from an ab-initio potential. Phys Chem Chem Phys 2008;10:5419-28.

[215] Thibault F, Cappelletti D, Pirani F, Bartolomei M. A bond-bond description of the intermolecular interaction energy: The case of the weakly bound acetylene-hydrogen complex. J Phys Chem A 2009;113:14867-74.

[216] Thibault F, Martinez RZ, Bermejo D, Ivanov SV, Buzykin OG, Ma Q. An experimental and theoretical study of nitrogen-broadened acetylene lines. J Quant Spectrosc Radiat Transf 2014;142:17-24.

[217] Cappelletti D, Bartolomei M, Carmona-Novillo E, Pirani F, Blanquet G, Thibault F. Intermolecular interaction potentials for $\mathrm{Ar}-\mathrm{C}_{2} \mathrm{H}_{2}, \mathrm{Kr}-\mathrm{C}_{2} \mathrm{H}_{2}$, and $\mathrm{Xe}-\mathrm{C}_{2} \mathrm{H}_{2}$ weakly bound complexes: Information from molecular beam scattering, pressure broadening coefficients, and rovibrational spectroscopy. J Chem Phys 2007; 126:064311.

[218] Buffa G, Tarrini O. Hyperfine effects on collisional lineshape. II. The case $\mathrm{DCO}^{+}-\mathrm{He}$. J Chem Phys 2011;134:174310.

[219] Hernández ML, Fernández JM, Tejeda G, Moreno E, Montero S. Broadening of $\mathrm{H}_{2} \mathrm{O}$ rotational lines by collisions with He atoms at low temperature. Astrophys J 2015;808:175.

[220] Buffa G, Dore L, Tinti F, Meuwly M. Experimental and theoretical study of helium broadening and shift of $\mathrm{HCO}^{+}$rotational lines. ChemPhysChem 2008;9:2237-44.

[221] Gomez L, Martinez RZ, Bermejo D, Thibault F, Joubert P, Bussery-Honvault B, Bonamy J. Q-branch linewidths of $\mathrm{N}_{2}$ perturbed by $\mathrm{H}_{2}$ : Experiments and quantum calculations from an $a b$-initio potential. $\mathrm{J}$ Chem Phys 2007;126:204302.

[222] Thibault F, Martinez RZ, Bermejo D, Gomez L. Collisional line widths of autoperturbed $\mathrm{N}_{2}$ : Measurements and quantum calculations. J Quant Spectrosc Radiat Transf 2011;112:2542-51.

[223] Buffa G, Tarrini O, Dore L, Meuwly M. Experimental and theoretical study of the broadening and shifting of $\mathrm{N}_{2} \mathrm{H}^{+}$rotational lines by helium. ChemPhysChem 2010;11:3141-5.

[224] Cherkasov MR. Theory of relaxation parameters of the spectrum shape in the impact approximation-I: General consideration. J Quant Spectrosc Radiat Transf 2014;141:73-88.

[225] Cherkasov MR. Theory of relaxation parameters of the spectrum shape in the impact approximation-II: 
Simplifications, application for ${ }^{\mathrm{q}} \mathrm{Q}(\mathrm{J}, \mathrm{K})$ doublets in the $\mathrm{v}_{1}$ band of $\mathrm{NH}_{3}$ self-broadening. $\mathrm{J}$ Quant Spectrosc Radiat Transf 2014;141:89-98.

[226] Starikov VI. Calculation of relaxation parameters of overlapping lines of the ammonia molecule pressure broadened by argon and helium. Opt Spectrosc 2013;114:15-24.

[227] Ma Q, Boulet C, Tipping RH. Refinement of the Robert-Bonamy formalism: Considering effects from the line coupling. J Chem Phys 2013;139:034305.

[228] Boulet C, Ma Q, Thibault F. Line interference effects using a refined Robert-Bonamy formalism: The test case of the isotropic Raman spectra of autoperturbed $\mathrm{N}_{2}$. J Chem Phys 2014;140:084310.

[229] Thibault F, Boulet C, Ma Q. Line coupling effects in the isotropic spectra of $\mathrm{N}_{2}$ : A quantum calculation at room temperature. J Chem Phys 2014;140:044303.

[230] Ma Q, Boulet C, Tipping RH. Two dimensional symmetric correlation functions of the $\tilde{S}$ operator and two dimensional Fourier transforms: Considering the line coupling for $\mathrm{P}$ and $\mathrm{R}$ lines of linear molecules. J Chem Phys 2014;140:104304.

[231] Ma Q, Boulet C. The relaxation matrix for symmetric tops with inversion symmetry. I. Effects of line coupling on self-broadened $v_{1}$ and pure rotational bands of $\mathrm{NH}_{3}$. J Chem Phys 2016;144:224303.

[232] Ma Q, Boulet C, Tipping RH. Vibrational dependence of line coupling and line mixing in selfbroadened parallel bands of $\mathrm{NH}_{3}$. J Quant Spectrosc Radiat Transf 2017;203:425-33.

[233] Pine AS, Markov VN. Self- and foreign-gas-broadened lineshapes in the $v_{1}$ band of $\mathrm{NH}_{3}$. J Quant Spectrosc Radiat Transf 2004;228:124-42.

[234] Ivanov SV, Buzykin OG. Classical calculation of self-broadening in $\mathrm{N}_{2}$ Ramanjspectra. Mol Phys 2008;106:1291-302.

[235] Povey C, Guillorel-Obregon M, Predoi-Cross A, Ivanov SV, Buzykin OG, Thibault F. Low pressure line shape study of nitrogen-perturbed acetylene transitions in the $v_{1}+v_{3}$ band over a range of temperatures. Can J Phys 2013;91:896-905.

[236] Ivanov SV, Buzykin OG. Precision considerations of classical and semiclassical methods used in collision line broadening calculations: Different linear molecules perturbed by argon. J Quant Spectrosc Radiat Transf 2013;119:84-94.

[237] Ivanov SV, Boulet C, Buzykin OG, Thibault F. Line mixing effects in isotropic Raman spectra of pure $\mathrm{N}_{2}$ : A classical trajectory study. J Chem Phys 2014;141:184306.

[238] Esteki K, Predoi-Cross A, Povey C, Ivanov S, Ghoufi A, Thibault F, Smith MAH. Room temperature self- and $\mathrm{H}_{2}$-broadened line parameters of carbon monoxide in the first overtone band: Theoretical and revised experimental results. J Quant Spectrosc Radiat Transf 2017;203:309-24.

[239] Gordon RG. Theory of the width and shift of molecular spectral lines in gases. J Chem. Phys 1966;44:3083-9.

[240] Gordon RG. Semiclassical theory of spectra and relaxation in molecular gases. J Chem Phys 1966;45:1649-55.

[241] Ivanov SV. Quasi-bound complexes in collisions of different linear molecules: Classical trajectory study of their manifestations in rotational relaxation and spectral line broadening. J Quant Spectrosc Radiat Transf 2016;177:269-82.

[242] Ma Q, Tipping RH, Boulet C. Irreducible correlation functions of the $\hat{S}$ matrix in the coordinate representation: Application in calculating Lorentzian half-widths and shifts. J Chem Phys 2006; 124:014109.

[243] Robert D, Bonamy J. Short range force effects in semiclassical molecular line broadening calculations. J de Physique 1979;40:923-43.

[244] Buldyreva J, Lavrentieva N, Starikov V. Collisional line broadening and shifting of atmospheric gases: A practical guide for line shape modelling by current semi-classical approaches. Imperial College Press, London, (2011).

[245] Predoi-Cross A, Holladay C, Heung H, Bouanich JP, Mellau GC, Keller R, Hurtmans DR. Nitrogenbroadened lineshapes in the oxygen A-band: Experimental results and theoretical calculations. J Mol Spectrosc 2008;251:159-75.

[246] Yang C, Buldyreva J, Gordon IE, Rohart F, Cuisset A, Mouret G, Bocquet R, Hindle F. Oxygen, nitrogen and air broadening of HCN lines at terahertz frequencies. J Quant Spectrosc Radiat Transf 2008;109:2857-68.

[247] Bouanich JP, Predoi-Cross A. Theoretical calculations for line-broadening and pressure-shifting in the $v_{1}+v_{2}+v_{4}+v_{5}$ band of acetylene over a range of temperatures. Mol Phys 2011;109:2071-81.

[248] Gamache RR, Lamouroux J, Laraia AL, Hartmann JM, Boulet C. Semi-classical calculations of halfwidths and line shifts for transitions in the 30012-00001 and 30012-00001 bands of $\mathrm{CO}_{2} \mathrm{I}$ : Collisions with $\mathrm{N}_{2}$. J Quant Spectrosc Radiat Transf 2012;113:976-90. 
[249] Lamouroux J, Gamache RR, Laraia AL, Hartmann JM, Boulet C. Semi-classical calculations of halfwidths and line shifts for transitions in the 30012-00001 and 30012-00001 bands of $\mathrm{CO}_{2} \mathrm{II}$ : Collisions with $\mathrm{O}_{2}$ and air. J Quant Spectrosc Radiat Transf 2012;113:991-1003.

[250] Lamouroux J, Gamache RR, Laraia AL, Hartmann JM, Boulet C. Semi-classical calculations of halfwidths and line shifts for transitions in the 30012-00001 and 30012-00001 bands of $\mathrm{CO}_{2}$ III: Self collisions. J Quant Spectrosc Radiat Transf 2012;113:1536-46.

[251] Dhyne M, Lepère M, Joubert P. Semiclassical line broadening calculations, using an ab initio potential energy surface, in Q-branch and S-branch of $\mathrm{N}_{2}$ perturbed by $\mathrm{H}_{2}$. J Raman Spectrosc 2012;43:2008-14.

[252] Rozario H, Garber J, Povey C, Hurtmans D, Buldyreva J, Predoi-Cross A. Experimental and theoretical study of $\mathrm{N}_{2}$-broadened acetylene line parameters in the $v_{1}+v_{3}$ band over a range of temperatures. Mol Phys 2012;110:2645-63.

[253] Hartmann JM, Rosenmann L, Perrin MY, Taine J. Accurate calculated tabulations of CO line broadening by $\mathrm{H}_{2} \mathrm{O}, \mathrm{N}_{2}, \mathrm{O}_{2}$, and $\mathrm{CO}_{2}$ in the 200-3000-K temperature range. Appl Opt 1988;27:3063-5.

[254] Rosenmann L, Hartmann JM, Perrin MY, Taine J. Accurate calculated tabulations of IR and Raman $\mathrm{CO}_{2}$ line broadening by $\mathrm{CO}_{2}, \mathrm{H}_{2} \mathrm{O}, \mathrm{N}_{2}, \mathrm{O}_{2}$ in the 300-2400-K temperature range. Appl Opt 1988;27:3902-7.

[255] Bonamy J, Robert D, Hartmann JM, Gonze ML, Saint-Loup R, Berger H. Line broadening, line shifting, line coupling effects on $\mathrm{N}_{2}-\mathrm{H}_{2} \mathrm{O}$ stimulated Raman spectra. J Chem Phys 1989;91:5916-25.

[256] Fanjoux G, Millot G, Saint-Loup R, Chaux R, Rosenmann L. Coherent anti-Stokes Raman spectroscopy study of collisional broadening in the $\mathrm{O}_{2}-\mathrm{H}_{2} \mathrm{O}$ Q branch. J Chem Phys 1994;101:1061-71.

[257] Buldyreva J, Guibet M, Eliet S, Hindle F, Mouret G, Bocquet R, Cuisset A. Theoretical and experimental studies of $\mathrm{CH}_{3} \mathrm{X}-\mathrm{Y}_{2}$ rotational lineshapes for atmospheric spectra modelling: Application to room temperature $\mathrm{CH}_{3} \mathrm{Cl}-\mathrm{O}_{2}$. Phys Chem Chem Phys 2011;13:20326-34.

[258] Buldyreva J, Rohart F. Experimental and theoretical studies of room temperature sub-millimetre $\mathrm{CH}_{3}{ }^{35} \mathrm{Cl}$ line shapes broadened by $\mathrm{H}_{2}$. Mol Phys 2012;110:2043-53.

[259] Predoi-Cross A, Devi VM, Sung K, Sinyakova T, Buldyreva J, Benner DC, Smith MAH, Mantz AW. Temperature dependences of $\mathrm{N}_{2}$-broadening and shift coefficients in the $v_{6}$ perpendicular band of ${ }^{12} \mathrm{CH}_{3}$ D. J Quant Spectrosc Radiat Transf 2015;163:120-41.

[260] Bray C, Jacquemart D, Lacome N, Guinet M, Cuisset A, Eliet S, Hindle F, Mouret G, Rohart F, Buldyreva J. Analysis of self-broadened pure rotational and rovibrational lines of methyl chloride at room temperature. J Quant Spectrosc Radiat Transf 2013;116:87-100.

[261] Nguyen L, Blanquet G, Buldyreva J, Lepère M. Measurements and calculations of ethylene linebroadening by argon in the $v_{7}$ band at room temperature. Mol Phys 2008;106:873-80.

[262] Solodov AM, Starikov VI. Helium-induced halfwidths and line shifts of water vapor transitions in the $v_{1}+v_{2}$ and $v_{2}+v_{3}$ bands. Mol Phys 2009; 107:43-51.

[263] Starikov VI. The calculation of the temperature dependence of He broadening coefficients of $\mathrm{H}_{2} \mathrm{~S}$ rotation lines. Atmos Ocean Opt 2012;25:321-7.

[264] Petrova TM, Solodov AM, Starikov VI, Solodov AA. Measurements and calculations of He-broadening and -shifting parameters of water vapor transitions of the $v_{1}+v_{2}+v_{3}$ band. Mol Phys 2012;110:1493-503.

[265] Petrova TM, Solodov AM, Solodov AA. Vibrational dependence of an intermolecular potential for $\mathrm{H}_{2} \mathrm{O}-\mathrm{He}$ system. J Quant Spectrosc Radiat Transf 2013;129:241-53.

[266] Petrova TM, Solodov AM, Solodov AA, Starikov VI. Measurements and calculations of Ar-broadening and shifting parameters of water vapor transitions of the $v_{1}+v_{2}+v_{3}$ and. J Quant Spectrosc Radiat Transf 2014;148:116-26.

[267] Petrova TM, Solodov AM, Solodov AA, Starikov VI. Broadening parameters of the $\mathrm{H}_{2} \mathrm{O}-\mathrm{He}$ collisional system for astrophysical applications. J Mol Spectrosc 2016;321:50-8.

[268] Brown LR, Humphrey CM, Gamache RR. $\mathrm{CO}_{2}$-broadened water in the pure rotation and $v_{2}$ fundamental regions. J Mol Spectrosc 2007;246:1-21.

[269] Buldyreva J, Nguyen L. Extension of the exact trajectory model to the case of asymmetric tops and its application to infrared nitrogen broadened linewidths of ethylene. Phys Rev A 2008;77:042720.

[270] Drouin BJ, Gamache RR. Temperature dependent air broadened linewidths of ozone rotational transitions. J Mol Spectrosc 2008;251:194-202.

[271] Bykov AD, Lavrentieva NN, Mishina TP, Sinitsa LN, Barber RJ, Tolchenov RN, Tennyson J. Water vapor line width and shift calculations with accurate vibration-rotation wave functions. J Quant Spectrosc Radiat Transf 2008;109:1834-44.

[272] Gomez L, Tran H, Perrin A, Gamache RR, Laraia A, Orphal J, Chelin P, Fellows CE, Hartmann JM. Some improvements of the $\mathrm{HNO}_{3}$ spectroscopic parameters in the spectral region from 600 to $950 \mathrm{~cm}^{-1}$. J Quant Spectrosc Radiat Transf 2009;110:675-86. 
[273] Buldyreva J. Lavrentieva N. Nitrogen and oxygen broadening of ozone infrared lines in the $5 \mu \mathrm{m}$ region: Theoretical predictions by semiclassical and semi-empirical methods. Mol Phys 2009;107:152736.

[274] Lavrentieva N, Osipova A, Buldyreva J. Calculations of ozone line shifting induced by $\mathrm{N}_{2}$ and $\mathrm{O}_{2}$ pressure. Mol Phys 2009;107:2045-51.

[275] Jacquemart D, Laraia A, Kwaba-Tchana F, Gamache RR, Lacome N. Formaldehyde around 3.5 and 5.7 $\mu \mathrm{m}$ measurement and calculation of self and $\mathrm{N}_{2}$ broadening coefficients. J. Quant. Spectrosc. Radiat Transf 2010;111:1209-22.

[276] Buldyreva JV, Mishina TP, Lavrentieva NN, Osipova AS. Calculation of coefficients of collisional line broadening of ozone spectral lines induced by pressure of atmospheric gases. Opt Spectrosc 2010;108:512-22.

[277] Ma Q, Tipping RH, Gamache RR. Uncertainties associated with theoretically calculated $\mathrm{N}_{2}$-broadened half-widths of $\mathrm{H}_{2} \mathrm{O}$ lines. Mol Phys 2010;108:2225-52.

[278] Gamache RR, Laraia AL, Lamouroux J. Half-widths, their temperature dependence, and line shifts for the HDO- $\mathrm{CO}_{2}$ collision system for applications to $\mathrm{CO}_{2}$-rich planetary atmospheres. Icarus 2011;213:720-30.

[279] Ma Q, Tipping RH, Lavrentieva NN. Causal correlation functions and Fourier transforms: Application in calculating pressure induced shifts. J Quant Spectrosc Radiat Transf 2012;113:936-50.

[280] Buldyreva J, Margulès L, Motiyenko RA, Rohart F. Speed dependence of $\mathrm{CH}_{3}{ }^{35} \mathrm{Cl}_{-} \mathrm{O}_{2}$ line-broadening parameters probed on rotational transitions: Measurements and semi-classical calculations. J Quant Spectrosc Radiat Transf 2013;130:304-14.

[281] Devi MV, Benner DC, Sung K, Crawford TJ, Gamache RR, Renaud CL, Smith MAH, Mantz AW, Vilanueva GL. Line parameters for $\mathrm{CO}_{2}$ broadening in the $v_{2}$ band of $\mathrm{HD}{ }^{16} \mathrm{O}$. J Quant Spectrosc Radiat Transf 2017; $187: 472-88$.

[282] Devi VM, Gamache RR, Vispoel B, Renaud CL, Benner DC, Smith MAH, Blake TA, Sams RL. Line shape parameters of air-broadened water vapor transitions in the $v_{1}$ and $v_{3}$ spectral region. $\mathrm{J}$ Mol Spectrosc in press.

[283] Antony BK, Gamache RR. Self-broadening of water vapor transitions via the complex Robert-Bonamy theory. J Quant Spectrosc Radiat Transf 2007;105:148-63.

[284] Ngo NH, Ibrahim N, Landsheere X, Tran H, Chelin P, Schwell M, Hartmann JM. Intensities and shapes of $\mathrm{H}_{2} \mathrm{O}$ lines in the near-infrared by tunable diode laser spectroscopy. J Quant Spectrosc Radiat Transf 2012;113:870-7.

[285] Devi VM, Benner DC, Sung K, Crawford TJ, Gamache RR, Renaud CL, Smith MAH, Mantz AW, Villanueva GL. Line parameters for $\mathrm{CO}_{2}$ - and self-broadening in the $\mathrm{v}_{1}$ band of $\mathrm{HD}^{16} \mathrm{O}$. J Quant Spectrosc Radiat Transf 2017;203:133-67.

[286] Devi VM, Benner DC, Sung K, Crawford TJ, Gamache RR, Renaud CL, Smith MAH, Mantz AW, Villanueva GL. Line parameters for $\mathrm{CO}_{2}$ - and self-broadening in the $v_{3}$ band of $\mathrm{HD}^{16} \mathrm{O}$. J Quant Spectrosc Radiat Transf 2017;203:158-74.

[287] Godon M, Bauer A, Gamache RR. The continuum of water vapor mixed with methane: Absolute absorption at $239 \mathrm{GHz}$ and linewidth calculations. J Mol Spectrosc 2000;202:293-302.

[288] Antony BK, Niles DL, Wroblewski SB, Humphrey CM, Gabard T, Gamache RR. $\mathrm{N}_{2^{-}}, \mathrm{O}_{2^{-}}$and airbroadened half-widths and line shifts for transitions in the $v_{3}$ band of methane in the 2726- to $3200-\mathrm{cm}^{-1}$ spectral region. J Mol Spectrosc 2008;251:268-81.

[289] Gabard T, Boudon V. Line broadening coefficient calculations for methane perturbed by nitrogen. J Quant Spectrosc Radiat Transf 2010;111:1328-43.

[290] Gabard T. Calculated line broadening parameters for methane perturbed by diatomic molecules. J Mol Spectrosc 2013;291:61-8.

[291] Gamache RR, Lacome N, Pierre G, Gabard T. Nitrogen broadening of $\mathrm{SF}_{6}$ transitions in the $v_{3}$ band. J Mol Struct 2001;599:279-92.

[292] Lévy A, Lacome N, Chackerian C Jr. Collisional line mixing. In Spectroscopy of the Earth's Atmosphere and Interstellar Medium, KN Rao and A Weber Eds, Academic Press, Boston (1992), pp. 261-337.

[293] Lavorel B, Millot G, Saint-Loup R, Berger H, Bonamy L, Bonamy J, Robert D. Study of collisional effects on band shapes of the $v_{1} / 2 v_{2}$ Fermi dyad in $\mathrm{CO}_{2}$ gas with stimulated Raman spectroscopy. I. Rotational and vibrational relaxation in the $2 v_{2}$ band. J Chem Phys 1990;93:2176-84.

[294] Cherkasov MR. Effects of collisional interference of lines in the spectra of symmetric top molecules: III. Broadening of rotational transitions with a hyperfine structure. Opt Spectrosc 2009;107:553-63.

[295] Cherkasov MR. Effects of hyperfine splitting of levels in the pressure broadening of the methyl chloride rotational transitions. Atmos Ocean Opt 2011;24:22-9. 
[296] Niro F, Boulet C, Hartmann JM. Spectra calculations in central and wing regions of $\mathrm{CO}_{2}$ IR bands between 10 and $20 \mu \mathrm{m}$. I: Model and laboratory measurements. J Quant Spectrosc Radiat Transf 2004;88:483-98.

[297] Boulet C, Ma Q, Tipping RH. Line mixing in parallel and perpendicular bands of $\mathrm{CO}_{2}$ : A further test of the refined Robert-Bonamy formalism. J Chem Phys 2015;143:124313.

[298] Ma Q, Boulet C, Tipping RH. Effects on calculated half-widths and shifts from the line coupling for asymmetric-top molecules. J Chem Phys 2014;140:244301.

[299] Boulet C, Ma Q. The relaxation matrix for symmetric tops with inversion symmetry. II. Line mixing effects in the $v_{1}$ band of $\mathrm{NH}_{3}$. J Chem Phys 2016;144:224304.

[300] Ma Q, Boulet C, Tipping RH. Relaxation matrix for symmetric tops with inversion symmetry: Line coupling and line mixing effects on $\mathrm{NH}_{3}$ lines in the $v_{4}$ band. J Chem Phys 2017;146:134312.

[301] Gordon RG, McGinnis RP. Intermolecular potentials and infrared spectra. J Chem Phys 1971;55:4898906.

[302] Buldyreva JV, Bonamy L. Non-Markovian energy corrected sudden model for the rototranslational spectrum of $\mathrm{N}_{2}$. Phys Rev A 1999;60:370-6.

[303] Daneshvar L, Buldyreva J. Extension of the non-Markovian Energy-Corrected Sudden model to the case of parallel and perpendicular infrared bands. J Chem Phys 2013;139:164107.

[304] Predoi-Cross A, Unni AV, Liu W, Schofield I, Holladay C, McKellar ARW, Hurtmans D. Line shape parameters measurement and computations for self-broadened carbon dioxide transitions in the 3001200001 and 30013-00001 bands, line mixing, and speed dependence. J Mol Spectrosc 2007;245:34-51.

[305] Predoi-Cross A, Liu W, Holladay C, Unni AV, Schofiel I, McKellar ARW, Hurtmans D. Line profile study of transition in the 30012-00001 and 30013-00001 bands of carbon dioxide perturbed by air. J Mol Spectrosc 2007;246:98-112.

[306] Predoi-Cross A, Hambrook K, Keller R, Povey C, Schofield I, Hurtmans D, Over H, Mellau GC. Spectroscopic line shape study of the self-perturbed oxygen A-band. J Mol Spectrosc 2008;248:85-110.

[307] Povey C, Predoi-Cross A. Computations of temperature dependences for line shape parameters in the $30012 \leftarrow 00001$ and $30013 \leftarrow 00001$ bands of pure $\mathrm{CO}_{2}$. J Mol Spectrosc 2009;257:187-99.

[308] Predoi-Cross A, Liu W, Murphy R, Povey C, Gamache RR, Laraia AI, McKellar ARW, Hurtmans DR, Devi VM. Measurement and computations for temperature dependences of self-broadened carbon dioxide transitions in the $30012 \leftarrow 00001$ and $30013 \leftarrow 00001$ bands. J Quant Spectrosc Radiat Transf 2010;111:1065-79.

[309] Lamouroux J, Tran H, Laraia AL, Gamache RR, Rothmann LS, Gordon IE, Hartmann JM. Updated database plus software for line-mixing in $\mathrm{CO}_{2}$ infrared spectra and their test using laboratory spectra in the 1.5-2.3 $\mu \mathrm{m}$ region J Quant Spectrosc Radiat Transf 2010;111:2321-31.

[310] Farooq A, Jeffries JB, Hanson RK. High-pressure measurements of $\mathrm{CO}_{2}$ absorption near $2.7 \mu \mathrm{m}$ : Line mixing and finite duration collision effects. J Quant Spectrosc Radiat Transf 2010;111:949-60.

[311] Tran H, Boulet C, Stefani S, Snels M, Piccioni G. Measurements and modeling of high pressure pure $\mathrm{CO}_{2}$ spectra from 750 to $8500 \mathrm{~cm}^{-1}$. I-Central and wing regions of the allowed vibrational bands. J Quant Spectrosc Radiat Transf 2011;112:925-36.

[312] Filippov NN, Asfin RE, Sinyakova TN, Grigoriev IM, Petrova TM, Solodov AM, Solodov AA, Buldyreva JV. Experimental and theoretical studies of $\mathrm{CO}_{2}$ spectra for planetary atmosphere modelling: Region 600-9650 $\mathrm{cm}^{-1} /$ and pressures up to $60 \mathrm{~atm}$. Phys Chem Chem Phys 2013;15:13826-34.

[313] Daneshyar L, Földes T, Buldyreva J, Vander Auwera J. Infrared absorption by pure $\mathrm{CO}_{2}$ near $3340 \mathrm{~cm}$ ${ }^{1}$ : Measurements and analysis of collisional coefficients and line-mixing effects at subatmospheric pressures. J Quant Spectrosc Radiat Transf 2014;149:258-74.

[314] Lamouroux J, Régalia L, Thomas X, Vander Auwera J, Gamache RR, Hartmann JM. CO line-mixing database and software update and its tests in the $2.1 \mu \mathrm{m}$ and $4.3 \mu \mathrm{m}$ regions. J Quant Spectrosc Radiat Transf 2015;151:88-96.

[315] Al Mashwood A, Predoi-Cross A, Devi VM, Rozario H, Billinghurst B. Measurement and computations of line shape parameters for the $12201 \leftarrow 03301,11101 \leftarrow 10002$ and $12201 \leftarrow 11102$ selfbroadened $\mathrm{CO}_{2}$ Q-branches. J Mol Spectrosc in press.

[316] Buldyreva JV, Gennadiev NA, Filippov NN. Line-mixing in absorption bands of linear molecules diluted in high-density rare gases: Measurements and modeling for OCS-He. J Chem Phys 2013;138:164117.

[317] Tran H, Boulet C, Hartmann JM. Line-mixing and collision induced absorption by oxygen in the Aband. Laboratory measurements, model, and tools for atmospheric spectra computations. J Geophys Res Atmos 2006;111:D15210.

[318] Tran H, Hartmann JM. An improved $\mathrm{O}_{2}$ A-band absorption model and its consequences for retrievals of photon paths and surface pressures. J Geophys Res Atmos 2008;113:D18104. 
[319] Makarov DS, Tretyakov MY, Boulet C. Line mixing in the 60-GHz atmospheric oxygen band: Comparison of the MPM and ECS model. J Quant Spectrosc Radiat Transf 2013;124:1-10.

[320] Makarov DS, Filippov NN, Tretyakov MY. Modeling the profile of the $60 \mathrm{GHz}$ absorption band of atmospheric oxygen using the memory function formalism. Opt Spectrosc 2008;105:7-13.

[321] Bréchignac P. Reorientation and pressure broadening of IR or MW lines: New results in $\mathrm{CH}_{3} \mathrm{~F}$. J Chem Phys 1982;76:3389-95.

[322] Koubek J, Boulet C, Perrin A, Urban Š. Hartmann JM. Line-mixing between rotational Stark components of $\mathrm{CH}_{3} \mathrm{~F}$ self-perturbed and perturbed by helium: Experimental results and IOS analysis. J Mol Spectrosc 2011;266:12-20.

[323] Lemaire V, Dore L, Cazzoli G, Buffa G, Tarrini O, Belli S. Broadening of $\mathrm{CH}_{3} \mathrm{~F}$ in presence of Stark fields. I. Self-broadening and self-shifting of isolated components. J Chem Phys 1997;106:8995-9003.

[324] Green S. Effect of nuclear hyperfine structure on microwave spectral pressure broadening. J Chem Phys 1988;88:7331-6.

[325] Flaud PM, Orphal J, Boulet C, Hartmann JM. Measurements and analysis of collisional line-mixing within nuclear hyperfine components of helium broadened HI lines. J Mol Spectrosc 2006;235:149-57.

[326] Buffa G, Tarrini O. Hyperfine effects on collisional lineshape. I. A self-consistent set of equations. J Chem Phys 2011;134:174309.

[327] Brunner TA, Pritchard D. Fitting laws for rotationally inelastic collisions. In Advances in chemical physics: Dynamics of the excited state, vol 50, pp. 589-651, KP Lawley Ed., Wiley, NY, (1982).

[328] Tran H, Jacquemart D, Mandin JY, Lacome N. Line mixing in the $v_{6}$ Q branches of self and nitrogenbroadened methyl bromide. J Quant Spectrosc Radiat Transf 2008;109:119-31.

[329] Gomez L, Tran H, Jacquemart D. Line mixing calculation in the $v_{6}$ Q-branches of $\mathrm{N}_{2}$-broadened $\mathrm{CH}_{3} \mathrm{Br}$ at low temperatures. J Mol Spectrosc 2009;256 35-40.

[330] Povey C, Predoi-Cross A, Hurtmans DR. Line shape study of acetylene transitions in the $v_{1}+v_{2}+v_{4}+v_{5}$ band over a range of temperatures. J Mol Spectrosc 2011;268:177-88.

[331] Bray C, Tran H, Jacquemart D, Lacome N. Line mixing in the ${ }^{\mathrm{Q}} \mathrm{Q}$ sub branches of the $v_{1}$ band of methyl chloride. J Quant Spectrosc Radiat Transf 2012;113:2182-8.

[332] Hashemi R, Predoi-Cross A, Dudaryonok AS, Lavrentieva NN, Vandaele AC, Vander Auwera J. CO 2 pressure broadening and shift coefficients for the 2-0 band of ${ }^{12} \mathrm{C}^{16} \mathrm{O}$. J Mol Spectrosc 2016;326:60-72.

[333] Tran H, Hartmann JM, Toon G, Brown LR, Frankenberg C, Warneke T, Spietz P, Hase F. The $2 v_{3}$ band of $\mathrm{CH}_{4}$ revisited with line mixing. Consequences for spectroscopy and atmospheric retrievals at 1.67 $\mu \mathrm{m}$. J Quant Spectrosc Radiat Transf 2010;111:1344-56.

[334] Tran H, Vander Auwera J, Landsheere X, Ngo NH, Pangui E, Morales SB, El Hamzaoui H, Capoen B, Bouazaoui M, Boulet C, Hartmann JM. Infrared light on molecule-molecule and molecule-surface collisions. Phys Rev A 2015;92:012707.

[335] Hadded S, Thibault F, Flaud PM, Aroui H, Hartmann JM. Experimental and theoretical study of line mixing in $\mathrm{NH}_{3}$ spectra. I. Scaling analysis of parallel bands perturbed by He. J Chem Phys 2002;116:7544-57.

[336] Hadded S, Thibault F, Flaud PM, Aroui H, Hartmann JM. Experimental and theoretical study of line mixing in $\mathrm{NH}_{3}$ spectra. II. Effect of the perturber in infrared parallel bands. J Chem Phys 2004;120:21723.

[337] Filippov NN, Tonkov MV. Semiclassical analysis of line mixing in the infrared bands of $\mathrm{CO}$ and $\mathrm{CO}_{2} . \mathrm{J}$ Quant Spectrosc Radiat Transf 1993;50:111-25.

[338] Spiering FR, Kiseleva MB, Filippov NN, Naus H, van Lieshout B, Weijenborg C, van der Zande WJ. Line mixing and collision induced absorption in the oxygen A-band using cavity ringdown spectroscopy. J Chem Phys 2010;133:114305.

[339] Spiering FR, Kiseleva MB, Filippov NN, van Lieshout B, van der Veen AMH, van der Zande WJ. The effect of collisions with nitrogen on absorption by oxygen in the A-band using cavity ring-down spectroscopy. Mol Phys 2011;109:535-42.

[340] Sala JP, Bonamy J, Robert D, Lavorel B, Millot G, Berger H. A rotational thermalisation model for the calculation of collisionally narrowed isotropic Raman scattering spectra _ application to the SRS $\mathrm{N}_{2}$ QBranch. Chem Phys 1986;106:427-39.

[341] Verzhbitskiy IA, Kouzov AP, Rachet F, Chrysos M. The isotropic spectrum of the $\mathrm{CO}_{2} \operatorname{Raman}_{2} v_{3}$ overtone: A line-mixing band shape analysis at pressures up to several tens of atmospheres. J Chem Phys 2011;134:224301.

[342] Verzhbitskiy IA, Kouzov AP, Rachet F, Chrysos M. The depolarized Raman $2 v_{3}$ overtone of $\mathrm{CO}_{2}$ : A line-mixing shape analysis. J Chem Phys 2011;134:194305.

[343] Kochanov VP. Line profiles for the description of line mixing, narrowing, and dependence of relaxation constants on speed. J Quant Spectrosc Radiat Transf 2011;112:1931-41. 
[344] Rosenkranz PW. Shape of the $5 \mathrm{~mm}$ oxygen band in the atmosphere. IEEE Trans Ant and Prop 1975;23:498-506.

[345] Hartmann JM, Boulet C, Tran H, Nguyen MT. Molecular dynamics simulations for $\mathrm{CO}_{2}$ absorption spectra. I. Line broadening and the far wing of the $v_{3}$ infrared band. J Chem Phys 2010;133:144313.

[346] Hartmann JM, Boulet C. Molecular dynamics simulations for $\mathrm{CO}_{2}$ spectra. III. Permanent and collisioninduced tensors contributions to light absorption and scattering. J Chem Phys 2011;134:184312.

[347] Hartmann JM, Boulet C, Jacquemart D. Molecular dynamics simulations for $\mathrm{CO}_{2}$ spectra. II. The far infrared collision-induced absorption band. J Chem Phys 2011;134:094316.

[348] Hartmann JM, Boulet C, Tran DD, Tran H, Baranov Y. Effect of humidity on the absorption continua of $\mathrm{CO}_{2}$ and $\mathrm{N}_{2}$ near $4 \mu \mathrm{m}$. Calculations, comparisons with measurements and consequences for atmospheric spectra. J Chem Phys 2018;148:054304.

[349] Kouzov AP. Rotational relaxation matrix for fast non-Markovian collisions. Phys Rev A 1999;60:29319.

[350] Daneshvar L, Buldyreva J. Line mixing in Raman scattering spectra of $\mathrm{CO}_{2}$ modelled by a nonMarkovian Energy-Corrected Sudden approach. Mol Phys 2012;110:2077-89.

[351] Bogdanova JV, Rodimova OB. Line shape in far wings and water vapor absorption in a broad temperature interval. J Quant Spectrosc Radiat Transf 2010;111:2298-307.

[352] Klimeshina TE, Petrova TM, Rodimova OB, Solodov AA, Solodov AM. $\mathrm{CO}_{2}$ absorption in band wings in near IR. Atmos Ocean Opt 2015;28:387-93.

[353] Klimeshina TE, Rodimova OB. Temperature dependence of the water vapor continuum absorption in the 3-5 $\mu \mathrm{m}$ spectral region. J Quant Spectrosc Radiat Transf 2013;119:77-83.

[354] Klimeshina TE, Rodimova OB. Water-vapor foreign-continuum absorption in the 8-12 and 3-5 $\mu \mathrm{m}$ atmospheric windows. J Quant Spectrosc Radiat Transf 2015;161:145-52.

[355] Tanaka T, Fukabori M, Sugita T, Yokota T, Kumazawa R, Watanabe T, Nakajima H. Line shape of the far-wing beyond the band head of the $\mathrm{CO}_{2} \nu_{3}$ band. J Mol Spectrosc 2008;252:185-9.

[356] Tran H, Turbet M, Chelin P, Landsheere X. Measurements and modelling of absorption by $\mathrm{CO}_{2}+\mathrm{H}_{2} \mathrm{O}$ mixtures in the spectral region beyond the $v_{3} \mathrm{CO}_{2}$ band head. Icarus 2018;306:116-21.

[357] Shine KP, Ptashnik IV, Rädel G. The water vapour continuum: Brief history and recent developments. Surv Geophys 2012;33:535-55.

[358] Clough SA, Kneizys FX, Davies RW. Line shape and the water vapor continuum. Atmos Res 1989;23:229-41.

[359] Mlawer EJ, Payne VH, Moncet J, Delamere JS, Alvarado MJ, Tobin DC. Development and recent evaluation of the MT_CKD model of continuum absorption. Phil Trans R Soc A 2012;370:2520-56.

[360] Mlawer EJ, Alvarado MJ, Gombos D, Iacono MJ, Cady-Pereira KE, Pernak R, Furtado J, Payne VH. A potpourri of updates to the AER Models. In preparation.

[361] Burch DE. Continuum absorption by $\mathrm{H}_{2} \mathrm{O}$. Report AFGL-TR-81-0300, Air Force Geophysics Laboratory, Hanscom AFB, MA, 1982.

[362] Burch DE, Alt RL. Continuum absorption by $\mathrm{H}_{2} \mathrm{O}$ in the $700-1200 \mathrm{~cm}^{-1}$ and $2400-2800 \mathrm{~cm}^{-1}$ windows. Report AFGL-TR-84-0128, Air Force Geophysics Laboratory, Hanscom AFB, MA, 1984.

[363] Burch DE, Alt RL. Continuum absorption by $\mathrm{H}_{2} \mathrm{O}$ in the $700-1200 \mathrm{~cm}^{-1}$ and $2400-2800 \mathrm{~cm}^{-1}$ windows. Air Force Geophysics Laboratory, Hanscomb AFB, MA, 1984.

[364] Cormier JG, Ciurylo R, Drummond JR. Cavity ringdown spectroscopy measurements of the infrared water yapor continuum. J Chem Phys 2002;116:1030-4.

[365] Newman SM, Green PD, Ptashnik IV, Gardiner TD, Coleman MD, McPheat RA, Smith KM. Airborne and satellite remote sensing of the mid-infrared water vapour continuum. Phil Trans R Soc A 2012;370:2611-36.

[366] Clough SA Iacono MJ, Moncet JL. Line-by-line calculations of atmospheric fluxes and cooling rates application to water vapor. J Geophys Res Atmos 1992;97:15761-85.

[367] Costa SMS, Shine KP. Outgoing longwave radiation due to directly transmitted surface emission. J Atmos Sci 2012;69:1865-70.

[368] Hinderling J, Sigrist MW, Kneubuhl FK. Laser photoacoustic spectroscopy of water-vapor continuum and line absorption in the $8 \mu \mathrm{m}$ and $14 \mu \mathrm{m}$ atmospheric window. Infrared Phys 1987;27:63-120.

[369] Cormier JG, Hodges JT, Drummond JR. Infrared water vapor continuum absorption at atmospheric temperatures. J Chem Phys 2005;122:114309.

[370] Baranov YI, Lafferty WJ, Ma Q, Tipping RH. Water-vapor continuum absorption in the $800-1250 \mathrm{~cm}^{-1}$ spectral region at temperatures from 311 to 363 K. J Quant Spectrosc Radiat Transf 2008;109:2291-302.

[371] Turner DD, Tobin DC, Clough SA, Brown PD, Ellingson RG, Mlawer, EJ, Knuteson RO, Revercomb, HE, Shippert TR, Smith WL, Shephard MW. The QME AERI LBLRTM: A closure experiment for downwelling high spectral resolution infrared radiance. J Atmos Sci 2004;61:2657-75.

[372] http://rtweb.aer.com/continuum_frame.html. 
[373] Ptashnik IV, McPheat RA, Shine KP, Smith KM, Williams RG. Water vapor self-continuum absorption in near-infrared windows derived from laboratory measurements. J Geophys Res Atmos 2011;116:D16305.

[374] Ptashnik IV, Petrova TM, Ponomarev YB, Shine KP, Solodov AA, Solodov AM. Near-infrared water vapour self-continuum at close to room temperature. J Quant Spectrosc Radiat Transf 2013;120:23-35.

[375] Ptashnik IV, Petrova TM, Ponomarev YB, Shine KP, Solodov AA, Solodov AM. Water vapor continuum absorption in near-IR atmospheric windows. Atmos Ocean Opt 2015;28:115-20.

[376] Mondelain D, Aradj A, Kassi S, Campargue A. The water vapour self-continuum by CRDS at room temperature in the $1.6 \mu \mathrm{m}$ transparency window. J Quant Spectrosc Radiat Transf 2013;130:381-91.

[377] Mondelain D, Manigand S, Kassi S, Campargue A. Temperature dependence of the water vapor selfcontinuum by cavity ring-down spectroscopy in the $1.6 \mu \mathrm{m}$ transparency window. J Geophys Res Atmos 2014;119:5625-39.

[378] Ventrillard I, Romanini D, Mondelain D, Campargue A. Accurate measurements and temperature dependence of the water vapor self-continuum absorption in the $2.1 \mu \mathrm{m}$ atmospheric window. J Chem Phys 2015;143:134304.

[379] Mondelain D, Vasilchenko S, Cermak P, Kassi S, Campargue A. The self- and foreign-absorption continua of water vapor by cavity ring-down spectroscopy near $2.35 \mu \mathrm{m}$. Phys Chem Chem Phys 2015;17:17762-77.

[380] Campargue A, Kassi S, Mondelain D, Vasilchenko S, Romanini D. Accurate laboratory determination of the near infrared water vapor self-continuum: A test of the MT_CKD model. J Geophys Res Atmos 2016;121:13180-203.

[381] Richard L, Vasilchenko S, Mondelain D, Ventrillard J, Romanini D, Campargue A. Water vapor selfcontinuum absorption measurements in the 4.0 and $2.1 \mu \mathrm{m}$ transparency windows. J Quant Spectrosc Radiat Transf 2017;201:171-9.

[382] Bicknell WE, Di Cecca S, Griffin MK, Swartz SD, Flusberg A. Search for low-absorption regimes in the 1.6 and 2.1 $\mu \mathrm{m}$ atmospheric windows. J Directed Ener 2006;2:151-61.

[383] Fulghum SF, Tilleman MM. Interferometric calorimeter for the measurement of water-vapor absorption. J Opt Soc Am B 1991;8:2401-13.

[384] Baranov YI, Lafferty WJ. The water-vapor continuum and selective absorption in the 3-5 $\mu \mathrm{m}$ spectral region at temperatures from 311 to 363 K. J Quant Spectrosc Radiat Transf 2011;112:1304-13.

[385] Burch DE, Gryvnak DA, Pembrook JD. Investigation of the absorption of infrared radiation by atmospheric gases: Water, nitrogen, nitrous oxide. Report AFCRL-71-0124, Air Force Cambridge Research Laboratories, Hanscom AFB, MA, 1971.

[386] Ptashnik IV, McPheat R, Shine KP, Smith KM, Williams RG. Water vapour foreign-continuum absorption in near-infrared windows from laboratory measurements. Phil Trans R Soc A 2012;370:2557-77.

[387] Baranov YI, Lafferty WJ. The water vapour self- and water-nitrogen continuum absorption in the 1000 and $2500 \mathrm{~cm}^{-1}$ atmospheric windows. Phil Trans R Soc A 2012;370:2578-89.

[388] Baranov YI. The continuum absorption in $\mathrm{H}_{2} \mathrm{O}+\mathrm{N}_{2}$ mixtures in the $2000-3250 \mathrm{~cm}^{-1}$ spectral region at temperatures from 326 to 363 K. J Quant Spectrosc Radiat Transf 2011;112:2281-6.

[389] Frommhold L.Collision Induced Absorption in Gases. Cambridge Monographs on Atomic, Molecular, and Chemical Physics, Cambridge University Press, Cambridge, (2006).

[390] Tabisz GC and Neuman MN Eds. Collision- and interaction-induced spectroscopy. Springer, Netherlands, (2012).

[391] Vigasin AA. Water vapor continuum: Whether collision-induced absorption is involved?. J Quant Spectrose Radiat Transf 2014;148:58-64.

[392] Karman/T, van der Avoird A, Groenenboom C. Collision-induced absorption with exchange effects and anisotropic interactions: Theory and application to $\mathrm{H}_{2}-\mathrm{H}_{2}$. J Chem Phys 2015;142:084305.

[393] Gustafsson M, Frommhold L, Bailly D, Bouanich JP, Brodbeck C. Collision-induced absorption in the rototranslational band of dense hydrogen gas. J Chem Phys 2003;119:12264.

[394] Karman T, Miliordos E, Hunt KLC, Groenenboom GC, van der Avoird A. Quantum mechanical calculation of the collision-induced absorption spectra of $\mathrm{N}_{2}-\mathrm{N}_{2}$ with anisotropic interactions. J Chem Phys 2015;142:084306.

[395] Karman T, van der Avoird A, Groenenboom AGC. Line-shape theory of the $\mathrm{X}_{3} \Sigma_{\mathrm{g}}{ }^{-} \rightarrow \mathrm{a}^{1} \Delta_{\mathrm{g}}, \mathrm{b}_{1} \Sigma_{\mathrm{g}}{ }^{+}$ transitions in $\mathrm{O}_{2}-\mathrm{O}_{2}$ collision-induced absorption. J Chem Phys 2017;147:084307.

[396] Karman T, Koenis MAJ, Banerjee A, Parker DH, Gordon IE, van der Avoird A, van der Zande WJ, Groenenboom GC. Mechanisms of collision-induced absorption for spin-forbidden transitions. Nature Chem in press.

[397] Karman T, van der Avoird A, Gerrit CG. Potential energy and dipole moment surfaces of the triplet states of the $\mathrm{O}_{2}\left(\mathrm{X}^{3} \Sigma_{\mathrm{g}}{ }^{-}\right)-\mathrm{O}_{2}\left(\mathrm{X}^{3} \Sigma_{\mathrm{g}}{ }^{-}, \mathrm{a}^{1} \Delta_{\mathrm{g}}, \mathrm{b}_{1} \Sigma_{\mathrm{g}}{ }^{+}\right)$complex. J Chem Phys 2017;147:084306. 
[398] Tabisz G. Intra-collision effects in the collision-broadening of spectral line profiles. Int Rev Atomic Molecular Physics 2010;1:53-61.

[399] Gustafsson M, Frommhold L, Li X, Hunt KLC. Roto-translational Raman spectra of pairs of hydrogen molecules from first principles. J Chem Phys 2009;130:164314.

[400] Głaz W, Bancewicz T, Godet JL, Gustafsson M, Haskopoulos A, Maroulis G. Effects of anisotropic interaction-induced properties of hydrogen-rare gas compounds on rototranslational Raman scattering spectra: Comprehensive theoretical and numerical analysis. J Chem Phys 2016;145:034303.

[401] Abel M, Frommhold L, Gustafsson M. Collision-induced absorption at wavelengths near $5 \mu \mathrm{m}$ by dense hydrogen gas. J Chem Phys 2009;131;181102.

[402] Abel M, Frommhold L, Li X, Hunt KLC. Collision-induced Absorption by $\mathrm{H}_{2}$ pairs: From hundreds to thousands of Kelvin. J Phys Chem A 2011;115:6805-12.

[403] Frommhold L, Abel M, Wang F, Gustafsson M, Li X, Hunt KLC. Infrared atmospheric emission and absorption by simple molecular complexes, from first principles. Mol Phys 2010;108:2265-72.

[404] Abel M, Frommhold L, Li X, Hunt KLC. Infrared absorption by collisional $\mathrm{H}_{2}-\mathrm{He}_{\text {complexes at }}$ temperatures up to $9000 \mathrm{~K}$ and frequencies from 0 to $20000 \mathrm{~cm}^{-1}$. J Chem Phys 2012;136;:044319.

[405] Abel M, Frommhold L. Note: Collision-induced infrared absorption by gaseous deuterium. J Chem Phys 2010;133:146101.

[406] Abel M, Frommhold L, Li X, Hunt KLC. Note: Computation of collision-induced absorption by dense deuterium-helium gas mixtures. J Chem Phys 2011;134:076101.

[407] Abel M, Frommhold L, Li X, Hunt KLC. Comparison of the calculated collision-induced absorption spectra by dense hydrogen-helium, deuterium-helium, and tritium-helium gas mixtures. J Atomic Molec Opt Phys 2011;2011:470530.

[408] El-Kader MSA, Godet JL, El-Sadek AA, Maroulis G. Spectrallíne shapes of collision-induced light scattering (CILS) and collision-induced absorption (CIA) using isotropic intermolecular potential for $\mathrm{H}_{2}$-Ar. Mol Phys 2017;115:2614-25.

[409] El-Kader MSA, Maroulis G. Spectral line shapes of collision-induced absorption (CIA) using isotropic intermolecular potential for mixtures of molecular hydrogen with helium. Chem Phys Lett 2017;684:141-7.

[410] El-Kader MSA, Maroulis G. An empirical multi-parameter anisotropic intermolecular potential, collision-induced absorption and predicted collision-induced light scattering spectra for $\mathrm{CF}_{4}-\mathrm{He}$. Chem Phys 2011;388:78-85.

[411] El-Kader MSA, Maroulis G. New insights into collision-induced rototranslational absorption and scattering spectra of gaseous methane at different temperatures. J Mol Spectrosc 2012;281:28-39.

[412] El-Kader MSA, Mostafa SI, Bancewicz T, Maroulis G. Spectral lineshapes of collision-induced absorption (CIA) and collision-induced light scattering (CILS) for molecular nitrogen using isotropic intermolecular potential. New insights and perspectives. Chem Phys 2014;440:127-34.

[413] El-Kader MSA. Theoretical calculation of the rototranslational collision-induced absorption (CIA) spectra in $\mathrm{O}_{2}-\mathrm{O}_{2}$ pairs. Zeitschrift für Physikalische Chemie 2016;230:1099-109.

[414] Oparin DV, Filippov NN, Grigoriev IM, Kouzov AP. Effect of stable and metastable dimers on collision-induced rototranslational spectra: Carbon dioxide-rare gas mixtures. J Quant Spectrosc Radiat Transf 2017;196:87-93.

[415] Gruszka M, Borysow A. Computer simulation of the far infrared collision induced absorption spectra of gaseous $\mathrm{CO}_{2}$. Mol Phys 1998;93:1007-16.

[416] Bussery-Honvault B, Hartmann JM. Ab initio calculations for the far infrared collision induced absorption by $\mathrm{N}_{2}$ gas. J Chem Phys 2014;140:054309.

[417] Hartmann JM, Boulet C, Toon G. Collision-induced absorption by $\mathrm{N}_{2}$ near $2.16 \mu \mathrm{m}$ : Calculations, model and consequences for atmospheric remote sensing. J Geophys Res Atmos 2017;122:2419-28.

[418] Baranov YI, Buryak IA, Lokshtanov SE, Lukyanchenko VA, Vigasin AA. $\mathrm{H}_{2} \mathrm{O}-\mathrm{N}_{2}$ collision-induced absorption band intensity in the region of the $\mathrm{N}_{2}$ fundamental: ab initio investigation of its temperature dependence and comparison with laboratory data. Phil Trans R Soc A 2012;370:2691-709.

[419] Shapiro MM, Gush HP. The collision-induced fundamental and first overtone bands of oxygen and nitrogen. Can J Phys 1966;44:949-63.

[420] Levine HB. Quantum theory of collision-induced absorption in rare-gas mixtures. Phys Rev 1967;160:159-69.

[421] Birnbaum G, Cohen ER. Theory of line shape in pressure-induced absorption. Can J Phys 1976;54:593602.

[422] Lokshtanov SE, Bussery-Honvault B, Vigasin AA. Extensive ab initio study of the integrated IR intensity in the $\mathrm{N}_{2}$ fundamental collision-induced band. Mol Phys 2008;106:1227-31. 
[423] Buryak IA, Kalugina YN, Vigasin AA. Ab initio and multipolar characterisation of the induced dipole surface for $\mathrm{CH}_{4}-\mathrm{CH}_{4}$ : Application to dipole-forbidden absorption in the Titan's atmosphere. J Mol Spectrosc 2013;291:102-7.

[424] Kalugina YN, Lokshtanov SE, Cherepanov VM, Vigasin AA. Ab initio 3D potential energy and dipole moment surfaces for the $\mathrm{CH}_{4}$-Ar complex: Collision-induced intensity and dimer content. J Chem Phys 2016;144:054304.

[425] El-Kader MSA, Maroulis G. Quantum spectral rototranslational collision-induced absorption (CIA) in $\mathrm{CO}_{2}$ and $\mathrm{CO}_{2}-\mathrm{Rg}$ pairs $(\mathrm{Rg}=\mathrm{He}$, Ar and $\mathrm{Xe})$ : An insightful analysis based on new empirical multiproperty isotropic intermolecular potentials. Chem Phys Lett 2017;670:95-101.

[426] Chrysos M, Kouzov AP, Egorova NI, Rachet F. Exact low-order classical moments in collision-induced bands by linear rotors: $\mathrm{CO}_{2}-\mathrm{CO}_{2}$. Phys Rev Lett 2008;100:133007.

[427] Leforestier C, Tipping RH, Ma Q. Temperature dependences of mechanisms responsible for the watervapor continuum absorption. II. Dimers and collision-induced absorption. J Chem Phys 2010;132:164302.

[428] Buryak I, Vigasin AA. Classical calculation of the equilibrium constants for true bound dimers using complete potential energy surface. J Chem Phys 2015;143:234304.

[429] Rothman LS, Gordon IE, Babikov Y, Barbe A, Benner DC, Bernath PF, Birk M, Bizzocchi L, Boudon V, Brown LR, Campargue A, Chance K, Cohen EA, Coudert LH, Devi VM, Drouin BJ, Fayt A, Flaud J-M, Gamache RR, Harrison JJ, Hartmann J-M, Hill C, Hodges JT, Jacquemart D, Jolly A, Lamouroux J, Le Roy RJ, Li G, Long DA, Lyulin OM, Mackie CJ, Massie ST, Mikhailenko S, Müller HSP, Naumenko OV, Nikitin AV, Orphal J, Perevalov V, Perrin A, Polovtseva ER, Richard C, Smith MAH, Starikova E, Sung K, Tashkun S, Tennyson J, Toon GC, Tyuterev VlG, Wagner G. The HITRAN2012 molecular spectroscopic database. J Quant Spectrosc Radiat Transf 2013:130:4-50.

[430] Gordon IE, Rothman LS, Hill C, Kochanov RV, Tan Y, Bernath PF, Birk M, Boudon V, Campargue A, Chance KV, Drouin BJ, Flaud JM, Gamache RR, Hodges JT, Jacquemart D, Perevalov VI, Perrin A, Shine KP, Smith MAH, Tennyson J, Toon GC, Tran H, Tyuterev VG, Barbe A, Császár AG, Devi VM, Furtenbacher T, Harrison JJ, Hartmann JM, Jolly A, Johnson TJ, Karman T, Kleiner I, Kyuberis AA, Loos J, Lyulin OM, Massie ST, Mikhailenko SN, Moazzen-Ahmadi N, Müller HSP, Naumenko OV, Nikitin AV, Polyansky OL, Rey M, Rotger M, Sharpe SW, Sung K, Starikova E, Tashkun SA, Vander Auwera J, Wagner G, Wilzewski J, Wcisło P, Yu S, Zak EJ. The HITRAN2016 Molecular Spectroscopic Database. J Quant Spectrosc Radiat Transf 2017;203:3-69. See also https://www.cfa.harvard.edu/hitran/.

[431] Jacquinet-Husson N, Armante R, Scott NA, Chédin A, Crépeau L, Boutammine C, Bouhdaoui A, Crevoisier C, Capelle V, Boonne C, Poulet-Crovisier N, Barbe A, Benner DC, Boudon V, Brown LR, Buldyreva J, Campargue A, Coudert LH, Devi VM, Down MJ, Drouin BJ, Fayt A, Fittschen C, Flaud JM, Gamache RR, Harrison JJ, Hill C, Hodnebrog Ø, Hu SM, Jacquemart D, Jolly A, Jiménez E, Lavrentieva NN, Liu AW, Lodi L, Lyulin OM, Massie ST, Mikhailenko S, Müller HSP, Naumenko OV, Nikitin A, Nielsen CJ, Orphal J, Perevalov VI, Perrin A, Polovtseva E, Predoi-Cross A, Rotger M, Ruth AA, Yu SS, Sung K, Tashkun SA, Tennyson J, Tyuterev VG, Vander Auwera J, Voronin BA, Makie A. The 2015 edition of the GEISA spectroscopic database. J Mol Spectrosc 2016;327:31-72. See also http://ether.ipsl.jussieu.fr/etherTypo/?id=950.

[432] Richard C, Gordon IE, Rothman LS, Abel M, Frommhold L, Gustafsson M, Hartmann JM, Hermans C, Lafferty WJ, Orton GS, Smith KM, Tran H. New section of the HITRAN database: Collision-induced absorption (CIA).J Quant Spectrosc Radiat Transf 2012;113:1273-85.

[433] Karman T, Baranov YI, Hartmann JM, Kurucz RL, Sun K, Sung K, Wishnow EH, Wordsworth R, Vigasin AA, Volkamer R, van der Zande WJ, Rothman LS, Gordon IE. Update of the HITRAN collision-induced absorption section. In preparation.

[434] Tasinato N, Pietropolli Charmet A, Stoppa P, Giorgianni S. Determination of the vinyl fluoride line intensities by TDL spectroscopy: the object oriented approach of Visual Line Shape Fitting Program to line profile analysis. Mol Phys 2010;108:677-85.

[435] Lisak D, Havey DK, Hodges JT. Spectroscopic line parameters of water vapor for rotation-vibration transitions near $7180 \mathrm{~cm}^{-1}$. Phys Rev A 2009;79:052507.

[436] Mendonca J, Strong K, Toon GC, Wunch D, Sung K, Deutscher NM, Griffith DWT, Franklin JE. Improving atmospheric $\mathrm{CO}_{2}$ retrievals using line mixing and speed-dependence when fitting highresolution ground-based solar spectra. J Mol Spectros 2016;323:15-27.

[437] Domysławska J, Wójtewicz S, Masłowski P, Cygan A, Bielska K, Trawiński RS, Ciuryło R, Lisak D. A new approach to spectral line shapes of the weak oxygen transitions for atmospheric applications. J Quant Spectrosc Radiat Transf 2016;169:111-21.

[438] Hartmann JM, Tran H. Comment on "Ortho-Para-Dependent Pressure Effects Observed in the Near Infrared Band of Acetylene by Dual-Comb Spectroscopy". Phys Rev Lett 2017;119:069401. 
[439] http://www.hitran.org/.

[440] Loos J, Birk M, Wagner G. Measurement of air-broadening line shape parameters and temperature dependence parameters of $\mathrm{H}_{2} \mathrm{O}$ lines in the spectral ranges $1850-2280 \mathrm{~cm}^{-1}$ and $2390-4000 \mathrm{~cm}^{-1}$. J Quant Spectrosc Radiat Transf 2017;203:103-18.

[441] Hodges JT, Lisak D, Lavrentieva N, Bykov A, Sinitsa L, Tennyson J, Barber RJ, Tolchenov RN. Comparison between theoretical calculations and high-resolution measurements of pressure broadening for near-infrared water spectra. J Mol Spectrosc 2008;249:86-94.

[442] Lisak D, Hodges JT. Low-uncertainty $\mathrm{H}_{2} \mathrm{O}$ line intensities fort he 930-nm region. J Mol Spectrosc 2008;249:6-13.

[443] Lisak D, Hodges JT. High-resolution cavity ring-down spectroscopy measurements of blended $\mathrm{H}_{2} \mathrm{O}$ transitions. Appl Phys B 2007;88:317-25.

[444] Osipov KY, Kapitanov VA, Protasevich AE, Pereslavtseva AA, Ponurovsky YY. Diode laser spectroscopy of $\mathrm{H}_{2}{ }^{16} \mathrm{O}$ spectra broadened by $\mathrm{N}_{2}$ and $\mathrm{He}$ in $1.39 \mu \mathrm{m}$ region. J Quant Spectrosc Radiat Transf 2014;142:1-8.

[445] Campbell HM, Havey DK. Pressure-broadening of water transitions near $7180 \mathrm{~cm}^{-1}$ by helium isotopes. Spectrochim Acta A 2013;109:232-8.

[446] Schroeder PJ, Cich MJ, Yang J, Swann WC, Coddington I, Newbury NR, Drouin BJ, Rieker GB. Broadband, high-resolution investigation of advanced absorption line shapes at high temperature. Phys Rev A 2017;96:022514.

[447] Loos J, Birk M, Wagner G. Measurement of positions, intensities and self-broadening line shape parameters of $\mathrm{H}_{2} \mathrm{O}$ lines in the spectral ranges $1850-2280 \mathrm{~cm}^{-1}$ and $2390-4000 \mathrm{~cm}^{-1}$. J Quant Spectrosc Radiat Transf 2017;203:119-32.

[448] Ptashnik I V, McPheat R, Polyansky OL, Shine KP, Smith KM. Intensities and self-broadening coefficients of the strongest water vapour lines in the 2.7 and $6.25 \mu \mathrm{m}$ absorption bands. J Quant Spectrosc Radiat Transf 2016;177:92-107.

[449] Koshelev MA, Golubiatnikov GY, Vilkov IN, Tretyakov MY. Line shape parameters of the 22-GHz water line for accurate modeling in atmospheric applications. J Quant Spectrosc Radiat Transf 2018;205:51-8.

[450] Hurtmans D, Henry A, Valentin A, Boulet C. Narrowing broadening and shifting parameters for R(2) and $\mathrm{P}(14)$ lines in the $\mathrm{HCl}$ fundamental band perturbed by $\mathrm{N}_{2}$ and rare gases from tunable diode laser spectroscopy. J Mol Spectrosc 2009;254:126-36.

[451] Le T, Fissiaux L, Lepère M, Tran H. Isolated line shape of methane with various collision partners. J Quant Spectrosc Radiat Transf 2016;185:27-36.

[452] Fissiaux L, Populaire JC, Blanquet G, Lepère M. Infrared spectroscopy at high temperature: $\mathrm{N}_{2}$ - and $\mathrm{O}_{2}$-broadening coefficients in the $v_{4}$ band of $\mathrm{CH}_{4}$. J Mol Spectrosc 2015;317:26-31.

[453] Fissiaux L, Delière Q, Blanquet G, Robert S, Vandaele AC, Lepère $\mathrm{M} . \mathrm{CO}_{2}$-broadening coefficients in the $v_{4}$ fundamental band of methane at room temperature and application to $\mathrm{CO}_{2}$-rich planetary atmospheres. J Mol Spectrosc 2014;297:35-40.

[454] Es-sebbar E, Farooq A. Intensities, broadening and narrowing parameters in the $v_{3}$ band of methane. J Quant Spectrosc Radiat Transf 2014;149:241-52.

[455] Devi VM, Benner DC, Sung K, Brown LR, Crawford TJ, Yu S, Smith MAH, Mantz AW, Boudon V, Ismail S. Spectral line parameters including line shapes in the $2 v_{3} \mathrm{Q}$ branch of ${ }^{12} \mathrm{CH}_{4}$. J Quant Spectrosc Radiat Transf 2016;177:152-69.

[456] Devi VM, Benner DC, Smith MAH, Mantz AW, Sung K, Crawford TJ, Predoi-Cross A. Self- and airbroadened line shape parameters in the $v_{2}+v_{3}$ band of ${ }^{12} \mathrm{CH}_{4}: 4500-4630 \mathrm{~cm}^{-1}$. J Quant Spectrosc Radiat Transf 2015;152:149-65.

[457] Devi VM, Benner DC, Sung K, Brown LR, Crawford TJ, Miller CE, Drouin BJ, Payne VH, Yu S, Smith MAH, Mantz AW, Gamache RR. Line parameters including temperature dependences of selfand air-broadened line shapes of ${ }^{12} \mathrm{C}^{16} \mathrm{O}_{2}: 1.6-\mu \mathrm{m}$ region. J Quant Spectrosc Radiat Transf 2016;177:117-44.

[458] Larcher G, Landsheere X, Schwell M, Tran H. Spectral shape parameters of pure $\mathrm{CO}_{2}$ transitions near $1.6 \mu \mathrm{m}$ by tunable diode laser spectroscopy. J Quant Spectrosc Radiat Transf 2015;164:82-8.

[459] Long DA, Bielska K, Lisak D, Havey DK, Okumura M, Miller CE, Hodges JT. The air-broadened, near-infrared $\mathrm{CO}_{2}$ line shape in the spectrally isolated regime: Evidence of simultaneous Dicke narrowing and speed dependence. J Chem Phys 2011;135:064308.

[460] Long DA, Wójtewicz S, Miller CE, Hodges JT. Frequency-agile, rapid scanning cavity ring-down spectroscopy (FARS-CRDS) measurements of the $(30012) \longleftarrow(00001)$ near-infrared carbon dioxide band. J Quant Spectrosc Radiat Transf 2015;161:35-40. 
[461] Devi VM, Benner DC, Miller CE, Predoi-Cross A. Lorentz half-width, pressure-induced shift and speed-dependent coefficients in oxygen-broadened $\mathrm{CO}_{2}$ bands at 6227 and $6348 \mathrm{~cm}^{-1}$ using a constrained multispectrum analysis. J Quant Spectrosc Radiat Transf 2010;111:2355-69.

[462] Kiseleva M, Mandon J, Persijn V, Harren FJM. Line strength measurements and relative isotopic ratio ${ }^{13} \mathrm{C} /{ }^{12} \mathrm{C}$ measurements in carbon dioxide using cavity ring down spectroscopy. J Quant Spectrosc Radiat Transf 2018;204:152-8.

[463] Rutkowski L, Masłowski P, Johansson AC, Khodabakhsh A, Foltynowicz A. Optical frequency comb Fourier transform spectroscopy with sub-nominal resolution and precision beyond the Voigt profile. J Quant Spectrosc Radiat Transf 2018;204:63-73.

[464] Casa G, Wehr R, Castrillo A, Fasci E, Gianfrani L. The line shape problem in the near-infrared spectrum of self-colliding $\mathrm{CO}_{2}$ molecules: Experimental investigation and test of semiclassical models. J Chem Phys 2009;130:184306.

[465] Bui TQ, Long DA, Cygan A, Sironneau VT, Hogan DW, Rupasinghe PM, Ciuryło R, Lisak D, Okumura M. Observations of Dicke narrowing and speed dependence in air-broadened $\mathrm{CO}_{2}$ lineshapes near $2.06 \mu \mathrm{m}$. J Chem Phys 2014;141:174301.

[466] Benner DC, Devi VM, Sung K, Brown LR, Miller CE, Payne VH, Drouin BJ, Yu S, Crawford TJ, Mantz AW, Smith MAH, Gamache RR. Line parameters including temperature dependences of air- and self-broadened line shapes of ${ }^{12} \mathrm{C}^{16} \mathrm{O}_{2}:$ 2.06- $\mu \mathrm{m}$ region. J Mol Spectrosc 2016;326:21-47.

[467] Delière Q, Fissiaux L, Lepère M. Absolute line intensities and self-broadening coefficients in the $v_{3}-v_{1}$ band of carbon dioxide. J Mol Spectros 2012;272:36-42.

[468] Owen K, Es-sebbar E, Farooq A. Measurements of $\mathrm{NH}_{3}$ linestrengths and collisional broadening coefficients in $\mathrm{N}_{2}, \mathrm{O}_{2}, \mathrm{CO}_{2}$, and $\mathrm{H}_{2} \mathrm{O}$ near $1103.46 \mathrm{~cm}^{-1}$. J Quant Spectrosc Radiat Transf 2013;121:5668.

[469] Triki M, Lemarchand C, Darquié B, Sow PLT, Roncin V, Chardonnet C, Daussy C. Speed-dependent effects in $\mathrm{NH}_{3}$ self-broadened spectra: Towards the determination of the Boltzmann constant. Phys Rev A 2012;85:062510.

[470] Loos J, Birk M, Wagner G. Pressure broadening, -shift, speed dependence and line mixing in the $v_{3}$ rovibrational band of $\mathrm{N}_{2} \mathrm{O}$. J Quant Spectrosc Radiat Transf 2015;151:300-9.

[471] Hoffman KJ, Davies PB. Pressure broadening coefficients of transitions in the $v_{5}$ band of methyl bromide from fitting to Voigt and Galatry line profiles. J Mol Spectrosc 2009;254:69-77.

[472] Dudaryonok AS, Lavrentieva NN, Buldyreva J, Margulès L, Motiyenko RA, Rohart F. Experimental studies, line-shape analysis and semi-empirical calculations of broadening coefficients for $\mathrm{CH}_{3}{ }^{35} \mathrm{Cl}_{-} \mathrm{CO}_{2}$ submillimeter transitions. J Quant Spectrosc Radiat Transf 2014;145:50-6.

[473] Gupta V, Rohart F, Margulès L, Motiyenko RA, Buldyreva J. Line-shapes and broadenings of rotational transitions of $\mathrm{CH}_{3}{ }^{35} \mathrm{Cl}$ in collision with $\mathrm{He}$, Ar and Kr. J Quant Spectrosc Radiat Transf 2015;161:85-94.

[474] Seleznev AF, Fedoseev GV, KoshelevMA, Tretyakov MY. Shape of collision-broadened lines of carbon monoxide. J Quant Spectrosc Radiat Transf 2015;161:171-9.

[475] Predoi-Cross A, Esteki K, Rozario H, Naseri H, Latif S, Thibault F, Devi VM, Smith MAH, Mantz AW. Theoretical and revisited experimentally retrieved He-broadened line parameters of carbon monoxide in the fundamental band. J Quant Spectrosc Radiat Transf 2016;184:322-40.

[476] Predoi-Cross A, Rohart F, Bouanich JP, Hurtmans DR. Xenon-broadened CO line shapes in the fundamental band at 349 K. Can J Phys 2009;87:485-98.

[477] Devi VM, Benner DC, Smith MAH, Mantz AW, Sung K, Brown LR, Predoi-Cross A. Spectral line parameters including temperature dependences of self- and air-broadening in the $2 \leftarrow 0$ band of CO at 2.3 $\mu \mathrm{m}$. J Quant Spectrosc Radiat Transf 2012;113:1013-33.

[478] Devi VM, Benner DC, Smith MAH, Mantz AW, Sung K, Brown LR. Spectral line parameters including temperature dependences of air-broadening for the $2 \leftarrow 0$ band of ${ }^{13} \mathrm{C}^{16} \mathrm{O}$ and ${ }^{12} \mathrm{C}^{18} \mathrm{O}$ at $2.3 \mu \mathrm{m}$. J Mol Spectrosc 2012;276-277:33-48.

[479] Wójtewicz S, Stec K, Masłowski P, Cygan A, Lisak D, Trawiński RS, Ciuryło R. Low pressure lineshape study of self-broadened CO transitions in the $(3 \leftarrow 0)$ band. J Quant Spectrosc Radiat Transf 2013;130:191-200.

[480] Kowzan G, Stec K, Zaborowski M, Wojtewicz S, Cygan A, Lisak D, Maslowski P, Trawinski RS. Line positions, pressure broadening and shift coefficients for the second overtone transitions of carbon monoxide in argon. J Quant Spectrosc Radiat Transf 2017;191:46-54.

[481] Devi VM, Benner DC, Kleiner I, Sams RL, Fletcher LN. Line shape parameters of $\mathrm{PH}_{3}$ transitions in the Pentad near 4-5 $\mu \mathrm{m}$ : Self-broadened widths, shifts, line mixing and speed dependence. J Mol Spectrosc 2014;302:17-33.

[482] Forthomme D, McRaven CP, Sears TJ, Hall GE. Argon-induced pressure broadening, shifting and narrowing in the $\mathrm{CN} \mathrm{A}^{2} \Pi-\mathrm{X}^{2} \Sigma^{+}(1-0)$ band. J Phys Chem A 2013;117:11837-46. 
[483] Fissiaux L, Dhyne M, Lepère M. Diode-laser spectroscopy: Pressure dependence of $\mathrm{N}_{2}$-broadening coefficients of lines in the $v_{4}+v_{5}$ band of $\mathrm{C}_{2} \mathrm{H}_{2}$. J Mol Spectrosc 2009;254:10-5.

[484] Sajid MB, Es-sebbar E, Farooq A. Measurements of line strengths, $\mathrm{N}_{2^{-}}$, Ar-, He and self-broadening coefficients of acetylene in the $v_{4}+v_{5}$ combination band using a cw quantum cascade laser. J Quant Spectrosc Radiat Transf 2014;148:1-12.

[485] Le T, Fissiaux L, Tran H, Lepère M. $\mathrm{O}_{2}$-broadening coefficients of acetylene lines in the $v_{4}+v_{5}$ band at room temperature. J Mol Spectrosc 2015:314:48-53.

[486] Hashemi R, Rozario H, Povey C, Predoi-Cross A. Line-shape models testing on six acetylene transitions in the $v_{1}+v_{3}$ band broadened by $\mathrm{N}_{2}$. J Quant Spectrosc Radiat Transf 2014;140:58-66.

[487] Cich MJ, McRaven CP, Lopez GV, Sears TJ, Hurtmans D, Mantz AW. Temperature-dependent pressure broadened line shape measurements in the $v_{1}+v_{3}$ band of acetylene using a diode laser referenced to a frequency comb. Appl Phys B 2012;109:373-84.

[488] Koshelev MA, Delahaye T, Serov EA, Vilkov IN, Boulet C, Tretyakov MY. Accurate modeling of the diagnostic 118-GHz oxygen line for remote sensing of the atmosphere. J Quant Spectrosc Radiat Transf 2017;196:78-86.

[489] Wójtewicz S, Cygan A, Masłowski P, Domysławska J, Lisak D, Trawiński RS, Ciuryło R. Spectral line shapes of self-broadened P-branch transitions of oxygen B band. J Quant Spectrosc Radiat Transf 2014;144:36-48.

[490] Domysławska J, Wójtewicz S, Masłowski P, Cygan A, Bielska K, Trawiński RS, Ciuryło R, Lisak D. Spectral line shapes and frequencies of the molecular oxygen B-band R-branch transitions. J Quant Spectrosc Radiat Transf 2015;155:22-31.

[491] Wójtewicz S, Lisak D, Cygan A, Domysławska J, Trawinski RS, Ciuryło R. Line-shape study of selfbroadened $\mathrm{O}_{2}$ transitions measured by Pound-Drever-Hall-locked frequency-stabilized cavity ring-down spectroscopy. Phys Rev A 2011;84:032511.

[492] Wójtewicz S, Masłowski P, Cygan A, Wcisło P, Zaborowski M, Piwiński M, Ciuryło R, Lisak D. Speed-dependent effects and Dicke narrowing in nitrogen-broadened oxygen. J Quant Spectrosc Radiat Transf 2015;165:68-75.

[493] Long DA, Havey DK, Okumura M, Miller CE, Hodges JT, O 2 A-band line parameters to support atmospheric remote sensing. J Quant Spectrosc Radiat Transf 2010;111:2021-36.

[494] Long DA, Robichaud ED, Hodges JT. Frequency-stabilized cavity ring-down spectroscopy measurements of line mixing and collision-induced absorption in the $\mathrm{O}_{2}$ A-band. J Chem Phys 2012;137:014307.

[495] Robichaud DJ, Hodges JT, Brown LR, Lisak D, Maslowski P, Yeung LY, Okumura M, Miller CE. Experimental intensity and lineshape parameters oft he oxygen A-band using frequency-stabilized cavity ring-down spectrocopy. J Mol Spectrosc 2008;248:1-13.

[496] Havey DK, Long DA, Okumura M, Miller CE, Hodges JT. Ultra-sensitive optical measurements of high-J transitions in the $\mathrm{O}_{2} \mathrm{~A}$-band. Chem Phys Lett 2009;483:49-54.

[497] Kapitanov VA, Osipov KY, Protasevich AE, Ponomarev YN. Collisional parameters of $\mathrm{N}_{2}$ broadened methane lines in the R9 multiplet of the $2 v_{3}$ band. Multispectrum fittings of the overlapping spectral lines. J Quant Spectrosc Radiat Transf 2012;113:1985-92.

[498] Osipov KY, Protasevich AE, Kapitanov VA, Ponurovskii YY. Collision parameters of $\mathrm{N}_{2}$-broadened methane lines in R5 multiplet of $2 v_{3}$ band. Multispectrum fitting of overlapping spectral lines. Appl Phys B 2012;106:725-32.

[499] Hashemi R, Predoi-Cross A, Nikitin AV, Tyuterev VG, Sung K, Smith MAH, Devi VM. Spectroscopic line parameters of ${ }^{12} \mathrm{CH}_{4}$ for atmospheric composition retrievals in the $4300-4500 \mathrm{~cm}^{-1}$ region. J Quant Spectrosc Radiat Transf 2017;186:106-17.

[500] Ghysels M, Gomez L, Cousin J, Tran H, Amarouche N, Engel A, Levin L, Durry G. Temperature dependences of air-broadening, air-narrowing and line-mixing coefficients of the methane $v_{3} R(6)$ manifold lines-Application to in-situ measurements of atmospheric methane. J Quant Spectrosc Radiat Transf 2014;133:206-16.

[501] Manne J, Bui TQ, Webster CR. Determination of foreign broadening coefficients for methane lines targeted by the tunable laser spectrometer (TLS) on the Mars curiosity rover. J Quant Spectrosc Radiat Transf 2017;191:59-66.

[502] Smith MAH, Benner DC, Predoi-Cross A, Devi VM. Air- and self-broadened half widths, pressureinduced shifts, and line mixing in the $v_{2}$ band of ${ }^{12} \mathrm{CH}_{4}$. J Quant Spectrosc Radiat Transf 2014;133:21734.

[503] Smith MAH, Benner DC, Predoi-Cross A, Malathy Devi V. Multispectrum analysis of ${ }^{12} \mathrm{CH}_{4}$ in the $\mathrm{v}_{4}$ spectral region: I. Air-broadened half widths, pressure-induced shifts, temperature dependences and line mixing. J Quant Spectrosc Radiat Transf 2009;110:639-53. 
[504] Smith MAH, Benner DC, Predoi-Cross A, Malathy Devi V. Multispectrum analysis of ${ }^{12} \mathrm{CH}_{4}$ in the $v_{4}$ spectral region: II. Self-broadened half widths, pressure-induced shifts, temperature dependences and line mixing. J Quant Spectrosc Radiat Transf 2010;111:1152-66.

[505] Smith MAH, Benner DC, Predoi-Cross A, Malathy Devi V. A multispectrum analysis of the $v_{4}$ band of ${ }^{13} \mathrm{CH}_{4}$ : Widths, shifts, and line mixing coefficients. J Quant Spectrosc Radiat Transf 2011;112:952-68.

[506] Devi VM, Benner DC, Brown LR, Miller CE, Toth RA. Line mixing and speed dependence in $\mathrm{CO}_{2}$ at $6348 \mathrm{~cm}^{-1}$ :positions, intensities, and air- and self-broadening derived with constrained multispectrum analysis. J Mol Spectrosc 2007;242:90-117.

[507] Devi VM, Benner DC, Brown LR, Miller CE, Toth RA. Line mixing and speed dependence in $\mathrm{CO}_{2}$ at $6227.9 \mathrm{~cm}^{-1}$ :constrained multispectrum analysis of intensities and line shapes in the 30013-00001 band. J Mol Spectrosc 2007;245:52-80.

[508] Christensen LE, Spiers GD, Menzies RT, Jacob JC. Tunable laser spectroscopy of $\mathrm{CO}_{2}$ near $2.05 \mu \mathrm{m}$ : Atmospheric retrieval biases due to neglecting line-mixing. J Quant Spectrosc Radiat Transf 2012;113:739-48.

[509] Smith MAH, Rinsland CP, Blake TA, Sams RL, Benner DC, Devi VM. Low-temperature measurements of HCN broadened by $\mathrm{N}_{2}$ in the $14-\mu \mathrm{m}$ spectral region. J Quant Spectrosc Radiat Transf 2008;109:922-51.

[510] Petrova TM, Solodov AM, Solodov AA. Line mixing in the water vapour transitions of the $v_{1}+v_{2}+v_{3}$ band perturbed by helium pressure. Mol Phys 2012;110:2071-5.

[511] Petrova TM, Solodov AM, Solodov AA, Starikov VL. Line mixing in some water vapor transitions perturbed by $\mathrm{N}_{2}$, Ar and He pressure. J Mol Struc 2015;1080:63-8.

[512] Tretyakov MN, Koshelev MA, Koval LA, Parshin VV, Kukin LM, Fedoseev LI, Dryagin YA, Andriyanov AF. Temperature dependence of pressure broadening of the $\mathrm{N}=1-$ fine structure oxygen line at 118.75 GHz. J Mol Spectrosc 2007;241:109-11.

[513] Rubens H, Aschkinass E. Beobachtungen über Absorption und Emission von Wasserdampf und Kohlensaüre im ultrarothen Spectrum. Ann Phys 1898;300:584-601.

[514] Hettner G. Über das ultrarote Absorptionsspektrum des Wasserdampfes. Ann Phys 1918;360:476-96.

[515] Camy-Peyret C, Vigasin AA (Eds). Weakly Interacting Molecular Pairs: Unconventional Absorbers of Radiation in the Atmosphere. NATO ARW Proceedings Series, Kluwer, Dordrecht, (2003).

[516] Ma Q, Tipping RH, Leforestier C. Temperature dependences of mechanisms responsible for the watervapor continuum absorption. I. Far wings of allowed lines. J Chem Phys 2008;128:124313.

[517] Ptashnik IV. Evidence for the contribution of water dimers to the near-IR water vapour self-continuum. J Quant Spectrosc Radiat Transf 2008;109:831-52.

[518] Ptashnik IV, Shine KP, Vigasin AA. Water vapour self-continuum and water dimers: 1. Analysis of recent work. J Quant Spectrose Radiat Transf 2011;112:1286-303.

[519] Tretyakov MY, Koshelev MA, Serov EA, Parshin VV, Odintsova TA, Bubnov GM. Water dimer and the atmospheric continuum. Uspekhi Fiz Nauk 2014;184:1199-215 (in Russian). English translation available in Physics - Uspekhi 2014;57:1083-98.

[520] Tretyakov MY. Spectroscopy underlying microwave remote sensing of atmospheric water vapor. J Mol Spectrosc 2016;328:7-26.

[521] Serov EA, Odintsova TA, Tretyakov MY, Semenov VE. On the origin of the water vapor continuum absorption within rotational and fundamental vibrational bands. J Quant Spectrosc Radiat Transf 2017;193:1-12.

[522] Odintsova TA, Tretyakov MY, Pirali O, Roy p. Water vapor continuum in the range of rotational spectrum of $\mathrm{H}_{2} \mathrm{O}$ molecule: New experimental data and their comparative analysis. J Quant Spectrosc Radiat Transf 2017;187:116-23.

[523] Tretyakov MN, Serov EA, Koshelev MA, Parshin VV, Krupnov AF. Water dimer rotationally resolved millimeter-wave spectrum observation at room temperature. Phys Rev Lett 2013;110:093001.

[524] Kiehl JY, Trenberth KE. Earth's annual global mean energy budget. Bull Am Meteorol Soc 1997;78:197-208.

[525] Held IM, Soden BJ. Water vapor feedback and global warming. Annu Rev Energy Environ 2000;25:441-75.

[526] Paynter DJ, Ramaswamy V. An assessment of recent water vapor continuum measurements upon longwave and shortwave radiative transfer. J Geophys Res Atmos 2011;116:D203302.

[527] Paynter DJ, Ramaswamy V. Variations in water vapor continuum radiative transfer with atmospheric conditions. J Geophys Res Atmos 2012;117:D16310.

[528] Paynter DJ, Ramaswamy V. Investigating the impact of the shortwave water vapor continuum upon climate simulations using GFDL global models. J Geophys Res Atmos 2014;119:10720-37. 
[529] Kopparapu RK, Ramirez R, Kasting JF, Eymet V, Robinson TD, Mahadevan S, Terrien RC, DomagalGoldman S, Meadows V, Deshpande R. Habitable zone around the main sequence stars: New estimates. Astrophys J 2013;765:131.

[530] Leconte J, Forget F, Charnay B, Wordsworth R, Selsis F, Millour E, Spiga A. 3D climate modeling of close-in land planets: Circulation patterns, climate moist bistability, and habitability. Astron Astrophys 2013;554:A69.

[531] Leconte J, Forget F, Charnay B, Wordsworth R, Pottier A. Increased insolation threshold for runaway greenhouse processes on Earth-like planets. Nature 2013;504:268-71.

[532] Continuum Absorption at Visible and Infrared wavelengths and its Atmospheric Relevance (CAVIAR). http://www.met.reading.ac.uk/caviar/home/.

[533] Podobedov VB, Plusquellic DF, Siegrist KE, Fraser GT, Ma Q, Tipping RH. New measurements of the water vapor continuum in the region from 0.3 to $2.7 \mathrm{THz}$. J Quant Spectrosc Radiat Transf 2008;109:458-67.

[534] Hartmann JM, Perrin MY, Ma Q, Tipping RH. The infrared continuum of pure water vapor calculations and high-temperature measurements. J Quant Spectrosc Radiat Transf 1993;49:675-91.

[535] Paynter DJ, Ptashnik IV, Shine KP, Smith KM, McPheat R, Williams RG. Laboratory measurements of the water vapor continuum in the $1200-8000 \mathrm{~cm}-1$ region between $293 \mathrm{~K}$ and $351 \mathrm{~K}$. J Geophys Res 2009;114:D21301.

[536] Aldener M, Brown SS, Stark H, Daniel JS, Ravishankara AR. Near-IR absorption of water vapor: Pressure dependence of line strengths and an upper limit for continuum absorption. J Mol Spectrosc 2005;232:223-30.

[537] Shine KP, Campargue A, Mondelain D, McPheat RA, Ptashnik IV, Weidmann D. The water vapour continuum in near-infrared windows - Current understanding and prospects for its inclusion in spectroscopic databases. J Mol Spectrosc 2016;327:193-208.

[538] Rocher-Casterline BE, Ch'ng LC, Mollner AK, Reisler H.Communication: determination of the bond dissociation energy $\left(\mathrm{D}_{0}\right)$ of the water dimer, $\left(\mathrm{H}_{2} \mathrm{O}\right)_{2}$, by velocity map imaging. J Chem Phys 2011;134:211101.

[539] Serio C, Masiello G, Esposito F, Di Girolamo P, Di Iorio T, Palchetti L, Bianchini G, Muscari G, Pavese G, Rizzi R, Carli B, Cuomo V. Retrieval of foreign-broadened water vapor continuum coefficients from emitted spectral radiance in the $\mathrm{H}_{2} \mathrm{O}$ rotational band from 240 to $590 \mathrm{~cm}^{-1}$. Opt Express 2008:16:15816-33.

[540] Liuzzi G, Masiello G, Serio C, Palchetti L, Bianchini G. Validation of $\mathrm{H}_{2} \mathrm{O}$ continuum absorption models in the wave number range $180-600 \mathrm{~cm}^{-1}$ with atmospheric emitted spectral radiance measured at the Antarctica Dome-C site. Opt Express 2014;22;16784-801.

[541] Reichert A, Rettinger M, Sussmann R. The Zugspitze radiative closure experiment for quantifying water vapor absorption over the terrestrial and solar infrared - Part 2: Accurate calibration of high spectral-resolution infrared measurements of surface solar radiation. Atmos Meas Tech 2016;9:4673-86.

[542] Reichert A, Sussmann R. The Zugspitze radiative closure experiment for quantifying water vapor absorption over the terrestrial and solar infrared - Part 3: Quantification of the mid- and near-infrared water vapor continuum in the 2500 to $7800 \mathrm{~cm}^{-1}$ spectral range under atmospheric conditions. Atmos Chem Phys 2016;16:11671-86.

[543] Sussmann R, Reichert A, Rettinger M. The Zugspitze radiative closure experiment for quantifying water vapor absorption over the terrestrial and solar infrared - Part 1: Setup, uncertainty analysis, and assessment of far-infrared water vapor continuum. Atmos Chem Phys 2016;16:11649-69.

[544] Brogniez H, English S, Mahfouf JF, Behrendt A, Berg W, Boukabara S, Buehler SA, Chambon P, Gambacorta A, Geer A, Ingram W, Kursinski ER, Matricardi M, Odintsova TA, Payne VH, Thorne PW, Tretyakov MY, Wang J. A review of sources of systematic errors and uncertainties in observations and simulations at $183 \mathrm{GHz}$. Atmos Meas Tech 2016;9:2207-21.

[545] Koshelev MA, Serov EA, Parshin VV, Tretyakov MY. Millimeter wave continuum absorption in moist nitrogen at temperatures 261-328 K. J Quant Spectrosc Radiat Transf 2011;112:2704-12.

[546] Reichert L, Andrés Hernández MD, Burrows JP, Tikhomirov AB, Firsov KM, Ptashnik IV. First CRDS-measurements of water vapour continuum in the $940 \mathrm{~nm}$ absorption band. J Quant Spectrosc Radiat Transf 2007;105:303-11.

[547] Tikhomirov AB, Ptashnik IV, Tikhomirov BA. Measurements of the continuum absorption coefficient of water vapour near $14400 \mathrm{~cm}^{-1}(0.694 \mu \mathrm{m})$. Opt Spectrosc 2006;101:80-9.

[548] Stefani S, Piccioni G, Snels M, Grassi D, Adriani A. Experimental $\mathrm{CO}_{2}$ absorption coefficients at high pressure and high temperature. J Quant Spectrosc Radiat Transf 2013;117:21-8.

[549] Baranov YI. On the significant enhancement of the continuum-collision induced absorption in $\mathrm{H}_{2} \mathrm{O}+\mathrm{CO}_{2}$ mixtures. J Quant Spectrosc Radiat Transf 2016;175:100-6. 
[550] Tonkov MV, Filippov NN, Bertsev VV, Bouanich JP, Nguyen Van Thanh, Brodbeck C, Hartmann JM, Boulet C, Thibault F, Le Doucen R. Measurements and empirical modelling of pure $\mathrm{CO}_{2}$ absorption in the $2.3 \mu \mathrm{m}$ region at room temperature: far wings, allowed and collision-induced bands. Appl Opt 1996;35:4863-70.

[551] Mondelain D, Vasilchenko S, Čermák P, Kassi A, Campargue A. The $\mathrm{CO}_{2}$ absorption spectrum in the $2.3 \mu \mathrm{m}$ transparency window by high sensitivity CRDS: (II) Self absorption continuum. J Quant Spectrosc Radiat Transf 2017;187:38-43.

[552] Kassi S, Campargue A, Mondelain D, Tran H. High pressure Cavity Ring Down Spectroscopy: Application to the absorption continuum of $\mathrm{CO}_{2}$ near $1.7 \mu \mathrm{m}$. J Quant Spectrosc Radiat Transf 2015;167:97-104.

[553] Mondelain D, Campargue A, Čermák P, Gamache RR, Kassi S, Tashkun SA, Tran H. The $\mathrm{CO}_{2}$ absorption continuum by high pressure CRDS in the $1.74 \mu \mathrm{m}$ window. J Quant Spectrosc Radiat Transf 2017;203:530-37.

[554] Snels M, Stefani S, Piccioni G, Bézard B. Carbon dioxide absorption at high densities in the $1.18 \mu \mathrm{m}$ nightside transparency window of Venus. J Quant Spectrosc Radiat Transf 2014;133:464-71.

[555] Baranov Y. Collision-induced absorption in the region of the $v_{2}+v_{3}$ band of carbon dioxide. J Mol Spectrosc in press.

[556] Stefani S, Piccioni G, Grassi D, Adriani A, Snels M. Temperature dependence of Collisional Induced Absorption (CIA) bands of $\mathrm{CO}_{2}$ with implications for Venus' atmosphere. J Quant Spectrosc Radiat Transf 2018;204:242-9.

[557] Baranov YI, Fraser GT, Lafferty WJ. Collision-induced absorption in the $\mathrm{CO}_{2}$ Fermi triad for temperatures from 211 to 296 K. In [494] , pp. 149-158;

[558] Abu-Kharma M, Omari HY, Shawaqfeld N, Stamp C. Collision induced absorption spectra of the fundamental band of $\mathrm{D}_{2}$ in binary mixtures $\mathrm{D}_{2}-\mathrm{Kr}$ at $298 \mathrm{~K}$. J Mol Spec 2010;259:111-5.

[559] Vasilchenko A, Mondelain D, Kassi S, Čermák P, Chomet B, Garnache A, Denet S, Lecocq V, Campargue A. The HD spectrum near $2.3 \mu \mathrm{m}$ by CRDS-VECSEL: Electric quadrupole transition and collision-induced absorption. J Mol Spectrosc 2016;326:9-16.

[560] Kassi S, Campargue A. Electric quadrupole transitions and collision-induced absorption in the region of the first overtone band of $\mathrm{H}_{2}$ near 1.25 $\mu \mathrm{m}$. J Mol Spectrosc 2014;300:55-9.

[561] Abu-Kharma M. Collision-induced absorption in the second overtone band of $\mathrm{H}_{2}$. Can J Phys 2012;90:339-43.

[562] Vangvichith M, Tran H, Hartmann JM. Line-mixing and collision induced absorption for $\mathrm{O}_{2}-\mathrm{CO}_{2}$ mixtures in the oxygen A band region. J Quant Spectrosc Radiat Transf 2009;110:2212-6.

[563] Spiering FR, Kiseleva MB, Filippov, van Kesteren L, van der Zande WJ. Collision-induced absorption in the $\mathrm{O}_{2}$ B-band region near $670 \mathrm{~nm}$. Phys Chem Chem Phys 2011;13:9616-21.

[564] Spiering FR, van der Zande WJ. Collision induced absorption in the $\mathrm{a}^{1} \Delta(v=2) \leftarrow \mathrm{X}^{3} \Sigma_{g}^{-}(v=0)$ band of molecular oxygen. Phys Chem Chem Phys 2012;14:9923-8.

[565] Thalman R, Volkamer R. Temperature dependent absorption cross-sections of $\mathrm{O}_{2}-\mathrm{O}_{2}$ collision pairs between 340 and $630 \mathrm{~nm}$ and at atmospherically relevant pressure. Phys Chem Chem Phys 2013;15:15371-9.

[566] Sneep M, Ityaksov D, Aben I, Linnartz H, Ubachs W. Temperature-dependent cross section of $\mathrm{O}_{2}-\mathrm{O}_{2}$ collision-nduced absorption resonances at 477 and $577 \mathrm{~nm}$. J Quant Spectrosc Radiat Transf 2006;98:405-24,

[567] Herrebout WA, van der Veken BJ, Kouzov AP, Filippov NN. Origin of abnormally sharp features in collision-induced spectra of cryosolutions. J Chem Phys 2015;143:044508.

[568] Hill C, Gordon IE, Rothman LS, Tennyson J. A new relational database structure and online interface for the HITRAN database. J Quant Spectrosc Radiat Transf 2013;130:51-61.

[569] Hill C, Gordon IE, Kochanov RV, Barrett L, Wilzewski JS, Rothman LS. HITRANonline: An online interface and the flexible representation of spectroscopic data in the HITRAN database. J Quant Spectrosc Radiat Transf 2016;177:4-14.

[570] Kochanov RV, Gordon IE, Rothman LS, Wcisło P, Hill C, Wilzewski JS. HITRAN Application Programming Interface (HAPI): A comprehensive approach to working with spectroscopic data. J Quant Spectrosc Radiat Transf 2016;177:15-30.

[571] Wilzewski JS, Gordon IE, Kochanov RV, Hill C, Rothman LS. $\mathrm{H}_{2}$, He, and $\mathrm{CO}_{2}$ line-broadening coefficients, pressure shifts and temperature-dependence exponents for the HITRAN database. Part 1: $\mathrm{SO}_{2}, \mathrm{NH}_{3}, \mathrm{HF}, \mathrm{HCl}, \mathrm{OCS}$ and $\mathrm{C}_{2} \mathrm{H}_{2}$. J Quant Spectrosc Radiat Transf 2016;168:193-206.

[572] Scott NA, Chédin A. A fast line-by-line method for atmospheric absorption computations: The Automatized Atmospheric Absorption Atlas. J Appl Meteor 1981;20:802-12. 
[573] Chéruy F, Scott NA, Armante R, Tournier B, Chédin A. Contribution to the development of radiative transfer models for high spectral resolution observations in the infrared. J Quant Spectrosc Radiat Transf 1995;53:597-611.

[574] http://4aop.noveltis.com/content/4aop-operational-release-4a-radiative-transfer-model.

[575] Armante R, Scott N, Crevoisier C, Capelle V, Crépeau L, Jacquinet N, Chédin A. Evaluation of spectroscopic databases through radiative transfer simulations compared to observations. Application to the validation of GEISA 2015 with IASI and TCCON. J Mol Spectrosc 2016;327:180-92.

[576] Infrared Atmospheric Sounding Interferometer (IASI). https://iasi-ng.cnes.fr/en/IASI-NG/index.htm, https://iasi.cnes.fr/en/IASI/index.htm and Refs. therein.

[577] MicroCarb (Carbon Dioxide Monitoring Mission). https://directory.eoportal.org/web/eoportal/satellitemissions/m/microcarb and Refs. therein.

[578] MERLIN (MEthane Remote Sensing LIdar Mission) Minisatellite. https://directory.eoportal.org/web/eoportal/satellite-missions/m/merlin and Refs. therein.

[579] Maté B, Lugez C, Fraser GT, Lafferty WJ Absolute intensities for the $\mathrm{O}_{2} 1.27 \mu \mathrm{m}$ continuum absorption. J Geophys Res Atmos 1999;104:30585-90.

[580] Thibault F, Menoux V, Le Doucen R, Rosenmann L, Hartmann JM, Boulet C. Infrared collision induced absorption by $\mathrm{O}_{2}$ near $6.4 \mu \mathrm{m}$ for atmospheric applications: Measurements and empirical modeling. Appl Opt 1997;36:563-7.

[581] Foucher PY. Détermination de profils verticaux de concentration en $\mathrm{CO}_{2}$ atmosphérique à partir d'observations spatiales. Application aux données en occultation solaire de l'instrument ACE-FTS sur SCISAT 1. PhD thesis, University Pierre et Marie Curie, Paris, (2009).

[582] Lafferty WJ, Solodov AM, Weber A, Olson WB, Hartmann JM. Infrared collision-induced absorption by $\mathrm{N}_{2}$ near $4.3 \mu \mathrm{m}$ for atmospheric applications: Measurements and empirical modeling. Appl Opt 1996;35:5911-7.

[583] Tran H, Flaud PM, Gabard T, Hase F, von Clarmann T, Camy-Peyret C, Payan S, Hartmann JM. Model, software, and database for line-mixing effects in the $v_{3}$ and $v_{4}$ bands of $\mathrm{CH}_{4}$ and tests using laboratory and planetary measurements. I. $\mathrm{N}_{2}$ (and air) broadenings and the Earth atmosphere. J Quant Spectrosc Radiat Transf 2006;101:284-305.

[584] GOSAT (Greenhouse gases Observing SATellite)/ Ibuki. https://directory.eoportal.org/web/eoportal/satellite-missions/g/gosat, and Refs. therein.

[585] OCO-2 (Orbiting Carbon Observatory-2). https://directory.eoportal.org/web/eoportal/satellitemissions/o/oco-2, and Refs. therein.

[586] TanSat (Chinese Carbon Dioxide Observation Satellite Mission); https://directory.eoportal.org/web/eoportal/satellite-missions/t/tansat, and Refs. therein.

[587] Active Sensing of $\mathrm{CO}_{2}$ Emissions over Nights, Days, \& Seasons (ASCENDS). See https://fpd.larc.nasa.gov/ascends.html and https://decadal.gsfc.nasa.gov/documents/10_ASCENDS.pdf.

[588] Eldering A, Wennberg PO, Crisp D, Schimel DS, Gunson MR, Chatterjee A, Liu J, Schwandner FM, Sun Y, O’Dell CW, Frankenberg C, Taylor T, Fisher B, Osterman GB, Wunch D, Hakkarainen J, Tamminen J, Weir B. The Orbiting Carbon Observatory-2 early science investigations of regional carbon dioxide fluxes. Science 2017;358:188-9.

[589] Durry G, Zéninari V, Parvitte B, Lefevre F, Ovarlez J, Gamache RR. Pressure-broadening coefficients and line strengths of $\mathrm{H}_{2} \mathrm{O}$ near $1.39 \mu \mathrm{m}$ : Application to the in situ sensing of the middle atmosphere with balloonborne diode lasers. J quant Spectrosc Radiat Transf 2005;94:387-403.

[590] Schneider M, Hase F, Blavier JF, Toon GC, Leblanc T. An empirical study on the importance of a speed-dependent Voigt line shape model for tropospheric water vapor profile remote sensing. J Quant Spectrosc Radiat Transf 2011;112:465-74.

[591] Tran H, Rohart F, Boone C, Eremenko M, Hase F, Bernath P, Hartmann JM. Non-Voigt line-shape effects on retrievals of atmospheric ozone: Collisionally isolated lines. J Quant Spectrosc Radiat Transf 2010;111:2012-20.

[592] Barret B, Hurtmans D, Carleer MR, De Mazière M, Mahieu E, Coheur PF. Line narrowing effect on the retrieval of $\mathrm{HF}$ and $\mathrm{HCl}$ vertical profiles from ground-based FTIR measurements. J Quant Spectrosc Radiat Transf 2005;95:499-519.

[593] Duchatelet P, Demoulin P, Hase F, Ruhnke R, Feng W, Chipperfield MP, Bernath PF, Boone CD, Walker KA, Mahieu F. Hydrogen fluoride total and partial column time series above the Jungfraujoch from long-term FTIR measurements: Impact of the line-shape model, characterization of the error budget and seasonal cycle, and comparison with satellite and model data. J Geophys Res Atmos 2010;115:D22306.

[594] Wunch D, Toon GC, Blavier JFL, Washenfelder RA, Notholt J, Connor BJ, Griffith DW, Sherlock V, Wennberg PO. The total carbon column observing network. Phil Trans R Soc A 2011;369:2087-112. See also http://www.tccon.caltech.edu/. 
[595] Mendonca J. Improving the Retrievals of Greenhouse Gases from Ground-Based Solar Absorption Spectra. PhD thesis, department of physics, Univertity of Toronto (2017).

[596] Washenfelder RA, Wennberg PO, Toon GC. Tropospheric methane retrieved from ground-based nearIR solar absorption spectra. Geophys Res Lett 2003;30:2226.

[597] Frankenberg C, Warneke T, Butz A, Aben I, Hase F, Spietz P, BrownLR. Pressure broadening in the $2 v_{3}$ band of methane and its implication on atmospheric retrievals. Atmos Chem Phys 2008,8:5061-75.

[598] Mendonca J, Strong K, Sung K, Devi VM, Toon GC, Wunch D, Franklin JE. Using high-resolution laboratory and ground-based solar spectra to assess $\mathrm{CH}_{4}$ absorption coefficient calculations. J Quant Spectrosc Radiat Transf 2017;190:48-59.

[599] Mondelain D, Camy-Peyret C, Deng W, Payan S, Mantz AW. Study of molecular line parameters down to very low temperature. Appl Phys B 2008;90:227-33.

[600] Hartmann JM, Tran H, Toon GC. Influence of line mixing on the retrievals of atmospheric $\mathrm{CO}_{2}$ from spectra in the 1.6 and 2.1 $\mu \mathrm{m}$ regions. Atmos Chem Phys 2009;9:7303-12.

[601] Thompson DR, Benner DC, Brown LR, Crisp D, Devi VM, Jiang Y, Natraj V, Oyafuso F, Sung K, Wunch D, Castaño R, Miller CE. Atmospheric validation of high accuracy $\mathrm{CO}_{2}$ absorption coefficients for the OCO-2 mission. J Quant Spectrosc Radiat Transf 2012;113:2265-76.

[602] Rinsland CP, Mahieu E, Demoulin P, Zander R, Servais C, Hartmann JM. Decrease of the carbon tetrachloride $\left(\mathrm{CCl}_{4}\right)$ loading above Jungfraujoch, based on high resolution infrared solar spectra recorded between 1999 and 2011. J Quant Spectrosc Radiat Transf 2012;113:1322-9.

[603] Tran H, Picquet-Varrault B, Boursier C, Viatte C, Eremenko M, Hase F, Hartmann JM. Non-Voigt lineshape effects on retrievals of atmospheric ozone: Line-mixing effects. J Quant Spectrosc Radiat Transf 2011;112:2287-95.

[604] Alvarado MJ, Payne VH, Mlawer EJ, Uymin G, Shephard MW, Cady-Pereira KE, Delamere JS, Moncet JL. Performance of the Line-By-Line Radiative Transfer Model (LBLRTM) for temperature, water vapor, and trace gas retrievals: recent updates evaluated with IASI case studies. Atmos Chem Phys 2013;13:6687-711.

[605] Payne VH, Mlawer E J, Cady-Pereira KE, Moncet JL. Water vapor continuum absorption in the microwave. IEEE Trans Geosci Remote Sensing 2011;49:2194-208.

[606] Coll C, Caselles V, Valor E, Niclòs R. Comparison between different sources of atmospheric profiles for land surface temperature retrieval from single channel thermal infrared data. Remote Sensing of Environment 2012;117:199-210.

[607] Delamere JS, Clough SA, Payne VH, Mlawer EJ, Turner DD, Gamache RR. A far-infrared radiative closure study in the Arctic: Application to water vapor. J Geophys Res Atmos 2010;115:D17106.

[608] Masiello G, Matricardi M, Serio C. The use of IASI data to identify systematic errors in the ECMWF forecasts of temperature in the upper stratosphere. Atmos Chem. Phys 2011;11:1009-21.

[609] Long DA, Hodges JT. On spectroscopic models of the $\mathrm{O}_{2}$ A-band and their impact upon atmospheric retrievals. J Geophy Res Atmos 2012;117:D12309.

[610] Foucher PY, Chédin A, Dufour G, Capelle V, Boone DC, Bernath P. Technical note: Feasibility of $\mathrm{CO}_{2}$ profile retrieval from limb viewing solar occultation made by the ACE-FTS instrument. Atmos Chem Phys 2009;9:2873-90.

[611] Foucher PY, Chédin A, Armante R, Boone DC, Crevoisier C, Bernath P. Carbon dioxide atmospheric vertical profiles retrieved from space observation using ACE-FTS solar occultation instrument. Atmos Chem Phys 2011;11:2455-70.

[612] O'Dell CW, Connor B, Bösch H, O'Brien D, Frankenberg C, Castano R, Christi M, Eldering D, Fisher B, Gunson M, McDuffie J, Miller CE, Natraj V, Oyafuso F, Polonsky I, Smyth M, Taylor T, Toon GC, Wennberg PO, Wunch D. The ACOS $\mathrm{CO}_{2}$ retrieval algorithm-Part 1: Description and validation against synthetic observations. Atmos Meas Tech 2012;5:99-121.

[613] Crisp D, Fisher BM, O'Dell C, Frankenberg C, Basilio R, Bösch H, Brown LR, Castano R, Connor B, Deutscher NM, Eldering A, Griffith D, Gunson M, Kuze A, Mandrake L, McDuffie J, Messerschmidt J, Miller CE, Morino I, Natraj V, Notholt J, O'Brien DM, Oyafuso F, Polonsky L, Robinson J, Salawitch R, Sherlock V, Smyth M, Suto H, Taylor TE, Thompson DR, Wennberg PO, Wunch D, Yung YL. The ACOS $\mathrm{CO}_{2}$ retrieval algorithm-Part II: Global XCO2 data characterization. Atmos Meas Tech 2012;5:687-707.

[614] Abshire JB, Riris H, Allan GR, Weaver CJ, Mao J, Sun X, Hasselbrack WE, Kawa SR, Biraud S. Pulsed airborne lidar measurements of atmospheric $\mathrm{CO}_{2}$ column absorption. Tellus B 2010;62:770-83.

[615] Sung K, Varanasi P. $\mathrm{CO}_{2}$-broadened half-widths and $\mathrm{CO}_{2}$-induced line shifts of ${ }^{12} \mathrm{C}^{16} \mathrm{O}$ relevant to the atmospheric spectra of Venus and Mars. J Quant Spectrosc Radiat Transf 2005;91:319-32.

[616] Lavrentieva NN, Voronin BA, Naumenko OV, Bykov AD, Fedorova AA. Linelist of HD ${ }^{16}$ O for study of atmosphere of terrestrial planets (Earth, Venus and Mars). Icarus 2014;236:38-47. 
[617] Tudorie M, Földes T, Vandaele AC, Vander Auwera J. $\mathrm{CO}_{2}$ pressure broadening and shift coefficients for the 1-0 band of $\mathrm{HCl}$ and DCl. J Quant Spectrosc Radiat Transf 2012;113:1092-101.

[618] Fedorova AA, Korablev OI, Bertaux JL, Rodin AV, Montmessin F, Belyaev DA, Reberac A. Solar infrared occultation observations by SPICAM experiment on Mars-Express: Simultaneous measurements of the vertical distributions of $\mathrm{H}_{2} \mathrm{O}, \mathrm{CO}_{2}$ and aerosol. Icarus 2009;200:96-117.

[619] Smith M, Wolff M, Clancy RT, Murchie S. Compact Reconnaissance Imaging Spectrometer observations of water vapor and carbon monoxide. J Geophys Res Planets 2009;114:E00D03.

[620] Krasnopolsky VA. Variations of the $\mathrm{HDO} / \mathrm{H}_{2} \mathrm{O}$ ratio in the martian atmosphere and loss of water from Mars. Icarus 2015;257:377-86.

[621] Fedorova A, Korablev O, Vandaele AC, Bertaux JL, Belyaev D, Mahieux A, Neefs E, Wilquet WV, Drummond R, Montmessin F, Villard E. HDO and $\mathrm{H}_{2} \mathrm{O}$ vertical distributions and isotopic ratio in the Venus mesosphere by Solar Occultation at Infrared spectrometer on board Venus Express. J Geophys Res Planets 2008;113:E00B22.

[622] Vandaele AC, De Mazière M, Drummond R, Mahieux A, Neefs E, Wilquet V, Korablev O, Fedorova A, Belyaev D, Montmessin F, Bertaux JL. Composition of the Venus mesosphere measured by Solar Occultation at Infrared on board Venus Express. J Geophys Res Planets 2008;113:E00B23.

[623] Krasnopolsky VA, Belyaev DA, Gordon IE, Li G, Rothman LS. Observations of D/H ratios in $\mathrm{H}_{2} \mathrm{O}$, $\mathrm{HCl}$, and HF on Venus and new DCl and DF line strengths. Icarus 2013;224:57-65.

[624] Fedorova AA, Trokhimovsky S, Korablev O, Montmessin F. Viking observation of water vapor on Mars: Revision from up-to-date spectroscopy and atmospheric models. Icarus 2010;208:156-64.

[625] Bézard B, Fedorova A, Bertaux JL, Rodin A, Korablev O. The 1.10- and 1.18- $\mu$ m nightside windows of Venus observed by SPICAV-IR aboard Venus Express. Icarus 2011;216:173-83.

[626] Fedorova A, Bézard B, Bertaux JL, Korablev O, Wilson C. The $\mathrm{CO}_{2}$ continuum absorption in the 1.10and 1.18- $\mu \mathrm{m}$ windows on Venus from Maxwell Montes transits by SPICAV IR onboard Venus express. Planet Space Sci 2015;113-114:66-77.

[627] Snels M, Stefani S, Grassi D, Piccioni G, Adriani A, Carbon dioxide opacity of the Venus' atmosphere. Planetary and Space Sciences;103:347-354.

[628] Hirtzig M, Bézard B, Lellouch E, Coustenis A, de Bergh C, Drossart P, Campargue A, Boudon V, Tyuterev V, Rannou P, Cours T, Kassi S, Nikitin A, Mondelain D, Rodriguez S, Le Mouélic S. Titan's surface and atmosphere from Cassini/VIMS data with updated methane opacity. Icarus 2013;226:47086.

[629] Rey M, Nikitin A, Bézard B, Rannou P, Coustenis A, Tyuterev VG. New accurate theoretical line lists of ${ }^{12} \mathrm{CH}_{4}$ and ${ }^{13} \mathrm{CH}_{4}$ in the 0-13400 $\mathrm{cm}^{-1}$ range: Application to the modeling of methane absorption in Titan's atmosphere. Icarus 2018;303:114-130.

[630] Devaraj K, Steffes PG, Duong D. The centimeter-wavelength opacity of ammonia under deep jovian conditions. Icarus 2014;241:165-79.

[631] Steffes PG, Hanley TR, Karpowicż BM, Devaraj K, Noorizadeh S, Duong D, Chinsomboon G, Bellotti A, Janssen M, Bolton SJ. High-precision laboratory measurements supporting retrieval of water vapor, gaseous ammonia, and aqueous ammonia clouds with the Juno Microwave Radiometer (MWR). Space Sci Rev 2016.

[632] Bellotti A, Steffes PG, Chinsomboon G. Laboratory measurements of the 5-20 cm wavelength opacity of ammonia, water vapor, and methane under simulated conditions for the deep jovian atmosphere. Icarus 2016;280:255-67.

[633] Bolton SJ, Adriani A, Adumitroaie V, Allison M, Anderson J, Atreya S, Bloxham J, Brown S, Connerney JEP, DeJong E, Folkner W, Gautier D, Grassi D, Gulkis S, Guillot T, Hansen C, Hubbard WB, Iess L, Ingersoll A, Janssen M, Jorgensen J, Kaspi Y, Levin SM, Li C, Lunine J, Miguel Y, Mura A, Orton G, Owen T, Ravine M, Smith E, Steffes P, Stone E, Stevenson D, Thorne R, Waite J, Durante D, Ebert RW, Greathouse TK, Hue V, Parisi M, Szalay JR, Wilson R. Jupiter's interior and deep atmosphere: The initial pole-to-pole passes with the Juno spacecraft. Science 2017;356:821-5.

[634] Li C, Ingersoll A, Janssen M, Levin S, Bolton S, Adumitroaie V, Allison M, Arballo J, Bellotti A, Brown S, Ewald S, Jewell L, Misra S, Orton G, Oyafuso F, Steffes P, Williamson R. The distribution of ammonia on Jupiter from a preliminary inversion of Juno microwave radiometer data. Geophys Res Lett 2017;44:5317-25.

[635] Rothman LS, Gordon IE, Barber RJ, Dothe H, Gamache RR, Goldman A, Perevalov VI, Tashkun SA, Tennyson J. HITEMP, the high-temperature molecular spectroscopic database. J Quant Spectrosc Radiat Transf 2010;111:2139-50. 
[636] Tennyson J, Yurchenko SN, Al-Refaie AF, Barton EJ, Chubb KL, Coles PA, Diamantopoulou S, Gorman MN, Hill C, Lam AZ, Lodi L, McKemmish LK, Na Y, Owens A, Polyansky OL, Rivlin T, Sousa-Silva C, Underwood DS, Yachmenev A, Zak E. The ExoMol database: Molecular line lists for exoplanet and other hot atmospheres. J Mol Spectrosc 2016;327:73-94. See also http://exomol.com/.

[637] Barton EJ, Hill C, Czurylo M, Li HY, Hyslop A, Yurchenko SN, Tennyson J. The ExoMol pressure broadening diet: $\mathrm{H}_{2}$ and He line-broadening parameters. J Quant Spectrosc Radiat Transf2017;203:4905.

[638] Hedges C, Madhusudhan N. Effect of pressure broadening on molecular absorption cross sections in exoplanetary atmospheres. Mon Notice Royal Astron Soc 2016;458:1427-49.

[639] Yurchenko SN, Tennyson J, Barton EJ. Molecular line shape parameters for exoplanetary atmospheric applications. J Phys Conf Series 2017;810:012010.

[640] Hanson RK. Applications of quantitative laser sensors to kinetics, propulsion and practical energy systems. Proc Combust Inst 2011;33:1-40.

[641] Bolshov MA, Kuritsyn YA, Romanovskii YV. Tunable diode laser spectroscopy as a technique for combustion diagnostics. Spectrochimica Acta B 2015;106:45-66.

[642] Goldenstein CS, Spearrin RM, Jeffries JB, Hanson RK. Infrared laser-absorption sensing for combustion gases. Prog Energy Combust Sci 2017;60:132-76.

[643] Johnson RH, Strandberg MWP. Broadening of microwave absorption lines by collisions with the cell walls. Phys Rev 1952;86:811-2.

[644] Danos M, Geschwind S. Broadening of microwave absorption lines due to wall collisions. Phys Rev 1953;91:1159-62.

[645] Ponomarev YN, Petrova TM, Solodov AM, Solodov AA. IR spectroscopy of water vapor confined in nanoporous silica aerogel. Opt Express 2010;18:26062-7.

[646] Svensson T, Shen Z. Laser spectroscopy of gas confined in nanoporous materials. Appl Phys Lett 2010;96:021107.

[647] Xu CT, Lewander M, Andersson-Engels S, Adolfsson E, Svensson T, Svanberg S. Wall-collision line broadening of molecular oxygen within nanoporous materials. Phys Rev A 2011:84:042705.

[648] Vander Auwera J, Ngo NH, Hamzaoui H, Capoen B, Bouazaoui M, Ausset P, Boulet C, Hartmann JM. Infrared absorption by molecular gases as a probe of nanoporous silica xerogel and molecule-surface collisions: Low pressure results. Phys Rev A 2013;88:042506.

[649] Hartmann JM, Vander Auwera J, Boulet C, Birot M, Dourges MA, ToupanceT, El Hamzaoui H, Ausset P, Carré Y, Kocon L, Capoen B, Bouazaoui M. Infrared absorption by molecular gases to probe porous materials and comparisons with other techniques. Micropor Mesopor Mat 2017;237:31-7.

[650] Svensson T, Adolfsson E, Burresi M, Savo R, Xu CT, Wiersma DS, Svanberg S. Pore size assessment based on wall collision broadening of spectral lines of confined gas:experiments on strongly scattering nanoporous ceramics with fine-tuned pore sizes. Appl Phys B 2013;110:147-54.

[651] Hartmann JM, Boulet C, Nander Auwera J, Hamzaoui H, Capoen B, Bouazaoui M. Line broadening of confined $\mathrm{CO}$ gas. From molecule-wall to molecule-molecule collisions with pressure. J Chem Phys 2014;140:064302.

[652] Hartmann JM, Landsheere X, Boulet C, Sarkisyan D, Sarkisyan AS, Leroy C, Pangui E. Infrared look at the spectral effects of submicron confinements of $\mathrm{CO}_{2}$ gas. Phys Rev A 2016;93:012516.

[653] Petrova TM, Ponomarev YN, Solodov AA, Solodov AM, Danilyuk AF. Spectroscopic nanoporometry of aerogel. JETP Lett 2015;101:65-7.

[654] Ponomarev YN, Petrova TM, Solodov AA, Solodov AM. Observation of a forbidden vibrational absorption band of $\mathrm{H}_{2}$ in nanoporous aerogel. JETP Lett 2014;99:619-21.

[655] Vander Auwera J, Boulet C, Carré Y, Kocon L, Hartmann JM. Confinement-induced infrared absorption by $\mathrm{H}_{2}$ and $\mathrm{N}_{2}$ gases in a porous silica aerogel. J Quant Spectrosc Radiat Transf 2016;182:193-8.

[656] Zhang XH, Khan A, Ducker WA. A nanosclae state. Phys Rev Lett 2007;98:136101.

[657] Zhang XH, Quinn A, Ducker WA. Nanobubbles at the interface between water and a hydrophobic solid. Langmuir 2008;24:4756-64.

[658] Fischer J. Low uncertainty Boltzmann constant determinations and the kelvin redefinition. Phil Trans R Soc A 2016;374:20150038.

[659] Gavioso RM, Madonna Ripa D, Steur PPM, Gaiser C, Zandt T, Fellmuth B, de Podesta M, Underwood R, Sutton G, Pitre L, Sparasci F, Risegari L, Gianfrani L, Castrillo A, Machin G. Progress towards the determination of thermodynamic temperature with ultra-low uncertainty. Phil Trans R Soc A 2016;374:20150046.

[660] Bordé CJ. Base units of the SI, fundamental constants and modern quantum physics. Phil Trans R Soc A 2005;363:2177-201. 
[661] Daussy C, Guinet M, Amy-Klein A, Djerroud K, Hermier Y, Briaudeau S, Bordé CJ, Chardonnet C. Direct determination of the Boltzmann constant by an optical method. Phys Rev Lett 2007;98:250801.

[662] Moretti L, Castrillo A, Fasci E, De Vizia M D, Casa G, Galzerano G, Merlone A, Laporta P and Gianfrani L. Determination of the Boltzmann constant by means of precision measurements of $\mathrm{H}_{2}{ }^{18} \mathrm{O}$ line shapes at $1.39 \mu \mathrm{m}$. Phys Rev Lett 2013;111:060803.

[663] Fasci E, De Vizia M D, Merlone A, Moretti L, Castrillo A, Gianfrani L. The Boltzmann constant from the $\mathrm{H}_{2}{ }^{18} \mathrm{O}$ vibration-rotation spectrum: complementary tests and revised uncertainty budget. Metrologia 2015;52:S233-41.

[664] Cygan A, Lisak D, Trawiński RS, Ciurylo R. Influence of the line-shape model on the spectroscopic determination of the Boltzmann constant. Phys Rev A 2010;82:032515.

[665] Casa G, Castrillo A, Galzerano G, Wehr R, Merlone A, Di Serafino D, Laporta P, Gianfrani L. Primary gas thermometry by means of laser-absorption spectroscopy: Determination of the Boltzmann constant. Phys Rev Lett 2008;100:200801.

[666] Lemarchand C, Triki M, Darquié B, Bordé CJ, Chardonnet C, Daussy C. Progress towards an accurate determination of the Boltzmann constant by Doppler spectroscopy. New J Phys 2011;13:073028.

[667] Hashemi R, Povey C, Derksen M, Naseri H, Garber J and Predoi-Cross A. Doppler broadening thermometry of acetylene and accurate measurement of the Boltzmann constant. J Chem Phys 2014;141:214201.

[668] Mejri S, Sow PLT, Kozlova O, Ayari C, Tokunaga SK, Chardonnet C, Briaudeau S, Darquié B, Rohart F, Daussy C. Measuring the Boltzmann constant by mid-infrared laser spectroscopy of ammonia. Metrologia 2015;52:S314-23.

[669] Amodio P, De Vizia MD, Moretti L, Gianfrani L. Investigating the ultimate accuracy of Doppler broadening thermometry by means of a global fitting procedure. Phys Rev A 2015;92:032506.

[670] Cheng CF, Wang J, Sun YR, Tan Y, Kang P, and Hu SM. Doppler broadening thermometry based on cavity ring-down spectroscopy. Metrologia 2015;52:S385-93.

[671] Gotti R, Moretti L, Gatti D, Castrillo A, Galzerano G, Laporta P, Gianfrani L, Marangoni M. A CavityRing-Down Doppler broadening thermometer. Phys Rev A 2018;97:012515.

[672] Zak E, Tennyson J, Polyansky OL, Lodi L, Zobov N, Tashkun S, Perevalov VI. A room temperature $\mathrm{CO}_{2}$ line list with ab initio computed intensities. J Quant Spectrosc Radiat Transf 2016;177:31-42.

[673] Huang X, Schwenke DW, Freedman RS, Lee TJ. Ames-2016 line lists for 13 isotopologues of $\mathrm{CO}_{2}$ : Updates, consistency, and remaining issues. J Quant Spectrosc Radiat Tranf 2017;203:224-41.

[674] Tashkun SA, Perevalov VI, Gamache RR, Lamouroux J. CDSD-296, high resolution carbon dioxide spectroscopic databank: Version for atmospheric applications. J Quant Spectrosc Radiat Transf 2015;152:45-73.

[675] Rhoderick GC, Duewer DL, Ning L, DeSirant K. Hydrocarbon gas standards at the pmol/mol level to support atmospheric measurements Anal Chem 2010;82:859-67.

[676] https://www.nist.gov/programs-projects/nist-traceable-reference-material-program-gas-standards.

[677] Kelley ME, Rhoderick GC, Guenther FR. Development and verification of air balance gas primary standards for the measurement of nitrous oxide at atmospheric levels. Anal Chem 2014;86:4544-9.

[678] Pascale C, Guillevic M, Ackérmann A, Leuenberger D, Niderhauser B. Two generators to produce SItraceable reference gas mixtures for reactive compounds at atmospheric levels. Meas Sci Tech 2017;28:124002.

[679] Nwaboh JA, Werhahn O, Ebert V. Line strength and collisional broadening coefficients of $\mathrm{H}_{2} \mathrm{O}$ at 2.7 $\mu \mathrm{m}$ for natural gas assurance applications. Mol Phys 2014;112:2451-61.

[680] Long DA, Okumura M, Miller CE, Hodges JT. Frequency-stabilized cavity ring-down spectroscopy measurements of carbon dioxide isotopic ratios. Appl Phys B 2011;105:471-7.

[681] Wójtewicz S, Cygan A, Masłowski P, Domysławska J, Wcisło P, Zaborowski M, Lisak D, Trawiński RS, Ciuryło R. Spectral line-shapes of oxygen B-band transitions measured with cavity ring-down spectroscopy. J Phys Conf Ser 2014;548:012028.

[682] Lisak D, Masłowski P, Cygan A, Bielska K, Wójtewicz S, Piwiński M, Hodges JT, Trawiński RS, Ciuryło R. Line shapes and intensities of self-broadened $\mathrm{O}_{2} \mathrm{~b}_{1} \Sigma_{\mathrm{g}}{ }^{+}(\mathrm{v}=1) \leftarrow \mathrm{X}_{3} \Sigma_{\mathrm{g}}{ }^{-}(\mathrm{v}=0)$ band transitions measured by cavity ring-down spectroscopy. Phys Rev A 2010;81:042504.

[683] Yi H, Liu Q, Gameson L, Fleisher AJ, Hodges JT. High-accuracy $\mathrm{CO}_{2}$ line intensities in the $2 \mu \mathrm{m}$ wavelength region measured by frequency-stabilized cavity ring-down spectroscopy. J Quant Spectrosc Radiat Transf 2018;206:367-77.

[684] Polyansky, OL, Bielska K, Ghysels M, Lodi L, Zobov N, Hodges JT, Tennyson J. High-accuracy $\mathrm{CO}_{2}$ line intensities determined from theory and experiment, Phys Rev Lett 2015;114:243001.

[685] Kerstel ERT, Iannone RQ, Chenevier M, Kassi S, Jost HJ, Romanini D. A water isotope $\left({ }^{2} \mathrm{H},{ }^{17} \mathrm{O}\right.$, and $\left.{ }^{18} \mathrm{O}\right)$ spectrometer based on optical feedback cavity-enhanced absorption for in situ airborne applications. Appl Phys B 2006;85:397-406. 
[686] Wu T, Chen W, Kerstel E, Gao X, Koeth J, Rössner K, Brückner D. Kalman Filtering real-time measurements of $\mathrm{H}_{2} \mathrm{O}$ isotopologue ratios by laser absorption spectroscopy at $2.73 \mu \mathrm{m}$. Opt Lett 2010;35:634-6.

[687] Castrillo A, Dinesan H, Casa G, Galzerano G, Laporta P and Gianfrani L. Amount-ratio determinations of water isotopologues by dual-laser absorption spectrometry. Phys Rev A 2012;86:052515.

[688] Kerstel E, Gianfrani L. Advances in laser-based isotope ratio measurements: selected applications. Appl Phys B 2008;92:439-49.

[689] Oyafuso F, Payne VH, Drouin BJ, Devi VM, Benner DC, Sung K, Yu S, Gordon IE, Kochanov R, Tan Y, Crisp D, Mlawer EJ, Guillaume A. High accuracy absorption coefficients for the Orbiting Carbon Observatory-2 (OCO-2) mission: Validation of updated carbon dioxide cross-sections using atmospheric spectra. J Quant Spectrosc Radiat Transf 2017;203:213-23.

[690] Taine J, Soufiani A. Gas IR radiative properties: From spectroscopic data to approximate models. Adv Heat Transf 1999;33:295-414.

[691] Rivière $\mathrm{P}$, Soufiani A. Updated band model parameters for $\mathrm{H}_{2} \mathrm{O}, \mathrm{CO}_{2}, \mathrm{CH}_{4}$ and $\mathrm{CO}$ radiation at high temperature. Int J Heat Mass Transf 2012;55:3349-58.

[692] Howell JR, Menguc MP, Siegel R. Thermal radiation heat transfer ( $6^{\text {th }}$ edition). Taylor and Francis, New York, ( 2015).

[693] Lee C, Richardson MI. A discrete ordinate, multiple scattering, radiative transfer model of the Venus atmosphere from 0.1 to $260 \mu \mathrm{m}$. J Atmos Sci 2011;68:1323-39.

[694] Lebonnois S, Eymet V, Lee C, Vatant d'Ollone J. Analysis of the radiative budget of the Venusian atmosphere based on infrared Net Exchange Rate formalism. J Geophys Res Planets 2015;120:1186200.

[695] Mendonça JM, Read PL, Wilson CE, Lee C. A new fast and flexible radiative transfer method for Venus general circulation models. Planet Space Sci 2015;105:80-93.

[696] Borysow A, Frommhold L. Theoretical collision-induced rototranslational absorption spectra for the outer planets: $\mathrm{H}_{2}-\mathrm{CH}_{4}$ pairs. Astrophys J 1986;304:849-65.

[697] Borysow A, Frommhold L. Collision-induced rototranslational absorption spectra of $\mathrm{CH}_{4}-\mathrm{CH}_{4}$ pairs at temperatures from 50 to $300 \mathrm{~K}$. Astrophys J 1987;318:940-3.

[698] Borysow A. Tang C. Far infrared CIA spectra of $\mathrm{N}_{2}-\mathrm{CH}_{4}$ pairs for modeling of Titan's atmosphere. Icarus 1993;105:175-83.

[699] Guerlet S, Spiga A, Sylvestre M, Indurain M, Fouchet T, Leconte J, Millour E, Wordsworth R, Capderou M, Bézard B, Forget F. Global climate modeling of Saturns atmosphere. Part I: Evaluation of the radiative transfer model. Icarus 2014.238:110-24.

[700] Forget F. Leconte J. Possible climates on terrestrial exoplanets. Phil Trans R Soc A 2014;372:20130084.

[701] Kasting J, Whitmire DP, Reynolds RT. Habitable zones around main sequence stars. Icarus 1993;101:108-28.

[702] Pollack JB, Kasting JF, Richardson, SM, Poliakoff K. The case for a wet, warm climate on early Mars. Icarus 1987;71:203-24.

[703] Kasting JF. CO $\mathrm{CO}_{2}$ condensation and the climate of early Mars. Icarus 1991;94:1-13.

[704] Forget F, Pierrehumbert RT.Warming early Mars with carbon dioxide clouds that scatter infrared radiation. Science 1997;278:1273-6.

[705] Pollack JB, Toon OB, Boese R. Greenhouse models of Venus' high surface temperature, as constrained by Pioneer Venus measurements. J Geophys Res Space Phys 1980;85:8223-31.

[706] Kasting JF, Pollack JB, Crisp D. Effects of high $\mathrm{CO}_{2}$ levels on surface temperature and atmospheric oxidation state of the early Earth. J Atmos Chem 1984;1:403-28.

[707] Ho W, Birnbaum G, Rosenberg A. Far-infrared collision-induced absorption in $\mathrm{CO}_{2}$. I. Temperature dependehce. J. Chem. Phys 1971;55:1028-38.

[708] Moore JF. Infrared absorption of carbon dioxide at high densities with application to the atmosphere of Venus. Technical Report X-630-72-48, NASA Goddard Space Flight Center, Greenbelt, Md. (1971).

[709] Wordsworth R, Forget F, Eymet V. Infrared collision-induced and far-line absorption in dense $\mathrm{CO}_{2}$ atmospheres. Icarus 2010;210:992-7.

[710] Baranov YI, Lafferty WJ, Fraser GT. Infrared spectrum of the continuum and dimer absorption in the vicinity of the $\mathrm{O}_{2}$ vibrational fundamental in $\mathrm{O}_{2} / \mathrm{CO}_{2}$ mixtures. J Mol Spectrosc 2004;228:432-40.

[711] Ozak N, Aharonson O, Halevy I. Radiative transfer in $\mathrm{CO}_{2}$-rich atmospheres: 1. Collisional line mixing implies a colder early Mars. J Geophys Res Planets 2016;121:965-85.

[712] Turbet M, Tran H. Comment on "Radiative transfer in $\mathrm{CO}_{2}$-rich atmospheres: 1. Collisional line mixing implies a colder early Mars”. J Geophys Res Planets 2017;122:2362-5.

[713] Forget F, Wordsworth R, Millour E, Madeleine J-B, Kerber L, Leconte J, Marcq E, Haberle RM. 3D modelling of the early martian climate under a denser $\mathrm{CO}_{2}$ atmosphere: temperatures and $\mathrm{CO}_{2}$ ice clouds. Icarus 2013;222:81-99. 
[714] Wordsworth R, Kalugina Y, Lokshtanov S, Vigasin A, Ehlmann B, Head J, Sanders C, Wang H. Transient reducing greenhouse warming on early Mars. Geophys Res Lett 2017;44:665-71.

[715] Ramirez RM, Kopparapu R, Zugger ME, Robinson TD, Freedman R, Kasting JF. Warming early Mars with $\mathrm{CO}_{2}$ and $\mathrm{H}_{2}$. Nature Geosci 2014;7:59-63.

[716] Marcq E. A simple 1-D radiative-convective atmospheric model designed for integration into coupled models of magma ocean planets. J Geophys Res Planets 2012;117:E01001.

[717] Segura TL, Toon OB, Colaprete A, Zahnle K. Environmental effects of large impacts on Mars. Science 2002;298:1977-80. 